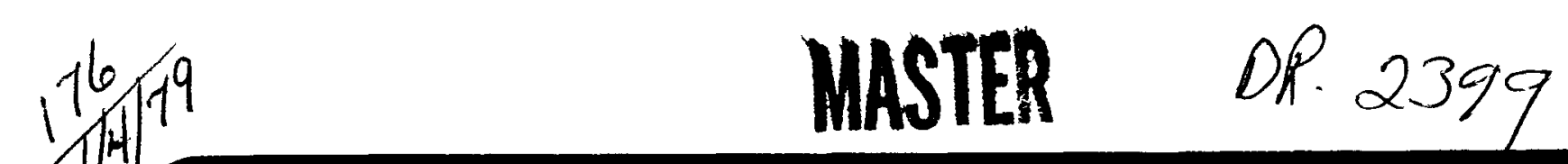

GA-A15116

UC-77

\title{
PROPERTIES OF UNIRRADIATED PRODUCTION GRADE H-451; LOT 478
}

by L_AREAVAN

Prepared under

Contract EY-76-C-03-0167

Project Agreement No. 65

for the San Francisco Operations Office

Department of Energy 


\section{NOTICE}

This report was prepared as an account of work sponsored by the United States Government. Neither the United States nor the United States Department of Energy, nor any of their employees, nor any of their contractors, subcontractors, or their employees, makes any warranty, express or implied, or assumes any legal liability or responsibility for the accuracy, completeness or usefulness of any information, apparatus, product or process disclosed, or represents that its use would not infringe privately owned rights.

Printed in the United States of America

Available from

National Technical Information Service

U.S. Department of Commerce

5285 Port Royal Road

Springfield, Virginia 22161

Price: Printed Copy $\$ 5.25$; Microfiche $\$ 3.00$ 


\section{DISCLAIMER}

This report was prepared as an account of work sponsored by an agency of the United States Government. Neither the United States Government nor any agency Thereof, nor any of their employees, makes any warranty, express or implied, or assumes any legal liability or responsibility for the accuracy, completeness, or usefulness of any information, apparatus, product, or process disclosed, or represents that its use would not infringe privately owned rights. Reference herein to any specific commercial product, process, or service by trade name, trademark, manufacturer, or otherwise does not necessarily constitute or imply its endorsement, recommendation, or favoring by the United States Government or any agency thereof. The views and opinions of authors expressed herein do not necessarily state or reflect those of the United States Government or any agency thereof. 


\section{DISCLAIMER}

Portions of this document may be illegible in electronic image products. Images are produced from the best available original document. 
GA-A15116

UC-77

\title{
PROPERTIES OF UNIRRADIATED PRODUCTION GRADE H-451; LOT 478
}

\author{
by \\ L. A. BEAVAN
}

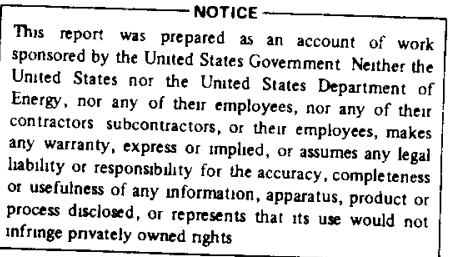

Prepared under

Contract EY-76-C-03-0167

Project Agreement No. 65

for the San Francisco Operations Office

Department of Energy

\section{GENERAL ATOMIC PROJECT 6422}

DATE PUBLISHED: JANUARY 1979 
0

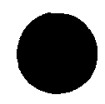




\section{ABSTRACT}

Three logs were selected from the first 98- log production lot of H-451 graphite. The three logs were characterized for density, mechanical properties under tensile, compressive, and flexural loads, thermal expansivity, thermal conductivity, and chemical impurity content.

Bulk density, thermal conductivity, and mechanical property values observed in the production-grade $\mathrm{H}-451$ were equal to or slightly (up to $5 \%)$ higher than comparable property values measured in preproduction grade $\mathrm{H}-451$, lot 426 . Thermal expansivity of the production $\mathrm{H}-451$ was as much as $10 \%$ lower than in preproduction material. Although chemical impurity content of the production grade logs was noticeably higher, the impurity levels remain within specified limits.

The weakest portion of the graphite log under axial stress was found to be the end center rather than the midlength center. 
0

0 
CONTENTS

ABSTRACT . . . . . . . . . . . . . . . . . . . . . 1 . . . . .

1. INTRODUCTION. . . . . . . . . . . . . . . . . . 1

2. MATERIALS . . . . . . . . . . . . . . . . . . 4 4

3. EXPERIMENTAL METHODS. . . . . . . . . . . . . . . 8

4. EXPERIMENTAL RESULTS . . . . . . . . . . . . . . 10

4.1. Bulk Density . . . . . . . . . . . . . . 10

4.2. Mechanical Properties ............. 10

4.2.1. Strength . . . . . . . . . . . . . 13

4.2.2. Elastic Modulus.............. 16

4.2.3. Poisson's Ratio.............. . 16

4.3. Thermal Properties ................ 18

4.3.1. Thermal Expansivity . . . . . . . . . 18

4.3.2. Thermal Conductivity ........... . 18

4.4. Chemical Purity . . . . . . . . . . . . . . 18

5. DISCUSSION AND CONCLUSIONS. . . . . . . . . . . . . . 22

ACKNOWLEDGMENTS . . . . . . . . . . . . . . . . . . . . . 24

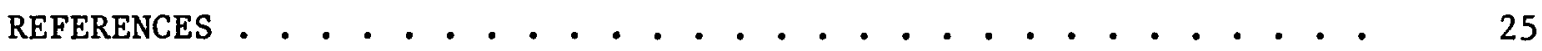

APPENDIX A: SAMPLING PLAN - MOT-007 . . . . . . . . . . A-1

APPENDIX B: INDIVIDUAL SPECIMEN DATA. . . . . . . . . . . B-1

FIGURES

1. Acceptance test core and characterization slabs for
production grade $\mathrm{H}-451$ graphite . . . . . . . . . . 6

2. Poisson's ratio specimen geometry . . . . . . . . . . 9

3. Rank of properties versus location in log . . . . . . . . 15 


\section{TABLES}

1. Properties of production grade $\mathrm{H}-451$ graphite . . . . . . . . 3

2. H-451 characterization logs . . . . . . . . . . . . . 5

3. Summary of property data: H-451, Lot 478 . . . . . . . . . . 11

4. Distribution of tensile strength in $\log 7194-52$. . . . . . . . 14

5. Poisson's ratios in H-451 graphite. . . . . . . . . . . . . . 17

6. Thermal conductivity of $\mathrm{H}-451$ graphite. . . . . . . . . . . . 19

7. H-451 impurity content, Lot 478 . . . . . . . . . . . . 20

8. Comparison of properties of production and preproduction H-451 graphite . . . . . . . . . . . . . . . . 23 


\section{INTRODUCTION}

Grade H-451 graphite has been developed by Great Lakes Carbon Co. (GLCC) for use as fuel elements and replaceable reflector elements in the high-temperature gas-cooled reactor (HTGR). Since development of production grade $\mathrm{H}-451$ from the first prototype grade $\mathrm{H}-429$ graphite has already been well documented by Engle (Ref. 1), it will be only briefly reviewed here.

GLCC developed two similar families of near-isotropic petroleum cokes, designated $A$ and $C$, as filler material for near-isotropic nuclear graphite. A prototype near-isotropic graphite, grade H-429, was first prepared from an A coke for preliminary irradiation experiments (Ref. 2). Subsequently, cokes $A$ and $C$ were separately blended with a regular production coke (B) to produce preproduction lots of grade $\mathrm{H}-451$ graphite, which were characterized by General Atomic (GA) for unirradiated properties (Refs. 3 and 4) and evaluated for irradiation performance (Refs. 5 and 6). Preproduction lot No. 426, manufactured with cokes $A_{3}$ and $B_{2}$, was selected as the basis for production of $\mathrm{H}-451$.

Production grade $\mathrm{H}-451$ graphite, made with cokes $A_{3}$ and $B_{2}$, was manufactured by GLCC in 1976 for use in the Fort St. Vrain reactor. Ninetyefght $\mathrm{H}-451$ logs were graphitized from three extrusion lots as part of an order for 354 logs. The balance of the 354 logs were processed through the bake stage and stored for future demand.

Al1 98 production $\mathrm{H}-451$ graphite logs were sampled along the $10 \mathrm{~g}$ centerline and tested for ultimate tensile strength (UTS) by GLCC and GA (Ref. 7). The same study (Ref. 7) also measured the UTS in a 25-mm-thick slab at the end of each log. The observed strength values and the deviations from $\log$ to $\log$ and $\operatorname{lot}$ to $\operatorname{lot}$ were used to evaluate acceptance criteria for $\mathrm{H}-451$ production. 
The 98 production logs were dispersed to GA, Oak Ridge Nationa1 Laboratory (ORNL), and Brookhaven Nationa1 Laboratory (BNL) for experimental purposes. Three logs were selected at GA for characterization of physical and chemical properties. The logs selected possessed nearly average density, slightly above-average tensile strength, and average to high ash (chemical impurity) content. This report presents the results of the characterization program and discusses these results in comparison to the strength acceptance testing (Ref. 7) and the characterization of preproduction H-451 (Refs. 3 and 4). Table 1 summarizes the properties of production grade $\mathrm{H}-451$ graphite. 
TABLE 1

PROPERTIES OF PRODUCTION GRADE H-451 GRAPHITE

\begin{tabular}{|c|c|c|c|}
\hline Property ${ }^{(a)}$ & Units & Axial & Radial \\
\hline Bulk density & $\left(\mathrm{Mg} / \mathrm{m}^{3}\right)$ & 1.74 & - \\
\hline \multicolumn{4}{|l|}{$\begin{array}{l}\text { Mechanical properties at midlength } \\
\text { center of } 10 \mathrm{~g}\end{array}$} \\
\hline Ultimate tensile strength & $(\mathrm{MPa})$ & 15.1 & 11.1 \\
\hline Modulus in tension & $(\mathrm{GPa})$ & 9.2 & 7.6 \\
\hline Flexura1 strength & $(\mathrm{MPa})$ & 23.5 & 18.7 \\
\hline Ultimate compressive strength & $(\mathrm{MPa})$ & 51.9 & 49.6 \\
\hline Modulus in compression & $(\mathrm{GPa})$ & 6.4 & 5.0 \\
\hline \multicolumn{4}{|l|}{ Poisson's ratios } \\
\hline$v_{12}$ & & & 0.150 \\
\hline$v_{13}$ & & & 0.117 \\
\hline$v_{31}=v_{32}$ & & 0.130 & \\
\hline \multicolumn{4}{|l|}{ Thermal conductivity } \\
\hline e $1073 \mathrm{~K}$ & $(W / m-K)$ & 64 & 59 \\
\hline $\begin{array}{l}\text { Mean coefficient of thermal } \\
\text { expansion }(293 \mathrm{~K}-773 \mathrm{~K})\end{array}$ & $\left(10^{-6} \mathrm{k}^{-1}\right)$ & 3.70 & 4.40 \\
\hline \multicolumn{4}{|l|}{ Impurity content } \\
\hline Ash & (ppm) & 230 & -- \\
\hline Boron & $(\mathrm{ppm})$ & 3 & - \\
\hline Iron & (ppm) & 30 & - \\
\hline
\end{tabular}

(a) Based upon complete characterization of three H-451 logs. 


\section{MATERIALS}

Production grade $\mathrm{H}-451$ graphite was extruded as 432-mm (17-in.) diam by $864-\mathrm{mm}$ (34-in.) long solid cylinders using conventional extrusion techniques. Near-isotropic petroleum cokes $A_{3}$ and $B_{2}$ were used as filler with coal-tar pitch binder and petroleum pitch impregnant. All 98 production logs were graphitized in one furnace run, although the green logs came from extrusion lots 472, 478, and 482 .

The three production logs selected for characterization studies were taken from extrusion lot 478. Properties measured by GLCC on each $10 \mathrm{~g}$ are presented in Table 2 with the GLCC serial number, GLCC 1 og number, and the GA assigned $\log$ number. Throughout this report, the GA $10 \mathrm{~g}$ number will be used to identify the graphite logs (7194-49, 7194-52, 7194-87).

A 16-mm (0.63-in.) diam, 356-mm (14-in.) long rod was taken along the axial centerline of each $10 \mathrm{~g}$ for acceptance strength testing studies (Fig. 1) (Ref. 7). The acceptance strength data reported by Engle and Price (Ref. 7) are included in Table 2.

Characterization specimens were taken from the three logs described in Table 2 following the sampling plan given in Appendix A. Each $10 \mathrm{~g}$ was sampled at four locations: end edge (EE) and end center (EC) (slab 1, Figs. 1 and A-1), midlength edge (MLE) and midlength center (MLC) (slab 3, Figs. 1 and A-2). Log 7194-52 was also sampled at the other end (slab 5, Fig. A-3) for mechanical property specimens. All specimens were taken as cylindrical cores and machined to appropriate specimen geometries.

The end slab taken for characterization work (slab 1, F1g. 1) was arbitrarily selected to be the opposite end from the location of the acceptance test core. Therefore, material from slabs 1 and 3 were tested 
TABLE 2

H-451 CHARACTERIZATION LOGS

\begin{tabular}{|c|c|c|c|c|c|c|c|c|}
\hline \multirow[b]{2}{*}{$\begin{array}{c}\text { GA } \\
\text { Log No. }\end{array}$} & \multirow[b]{2}{*}{$\begin{array}{c}\text { GLCC } \\
\text { Serial No. }\end{array}$} & \multirow[b]{2}{*}{$\begin{array}{c}\text { GLCC } \\
\text { Lot/Log No. }\end{array}$} & \multicolumn{5}{|c|}{ Properties Measured by GLCC } & \multirow{2}{*}{$\begin{array}{c}\mathrm{GA} \\
\mathrm{UTS}(\mathrm{b}) \\
(\mathrm{MPa})\end{array}$} \\
\hline & & & $\begin{array}{l}\text { Density } \\
\left(\mathrm{Mg} / \mathrm{m}^{3}\right)\end{array}$ & $\begin{array}{c}\text { Ash } \\
(\mathrm{ppm})\end{array}$ & $\begin{array}{c}\mathrm{Fe} \\
(\mathrm{ppm})\end{array}$ & $\begin{array}{c}\mathrm{V} \\
(\mathrm{ppm})\end{array}$ & $\begin{array}{r}\operatorname{UTS}^{(a)} \\
(\mathrm{MPa})\end{array}$ & \\
\hline $7194-49$ & 10084 & $478 / 15$ & 1.75 & 65 & -- & -- & 17.0 & 14.2 \\
\hline $7194-52$ & 10086 & $478 / 72$ & 1.73 & 115 & 11 & 7.5 & 13.4 & 16.1 \\
\hline $7194-87$ & 10145 & $478 / 35$ & 1.74 & 436 & 15 & 159 & 16.2 & 16.8 \\
\hline Mean of & 8 production & logs & 1.736 & & & & 13.4 & 12.8 \\
\hline
\end{tabular}

(a) One specimen per 10g. Near axial EC.

(b) Four specimens per log. Near axial MLC (Ref. 7). 


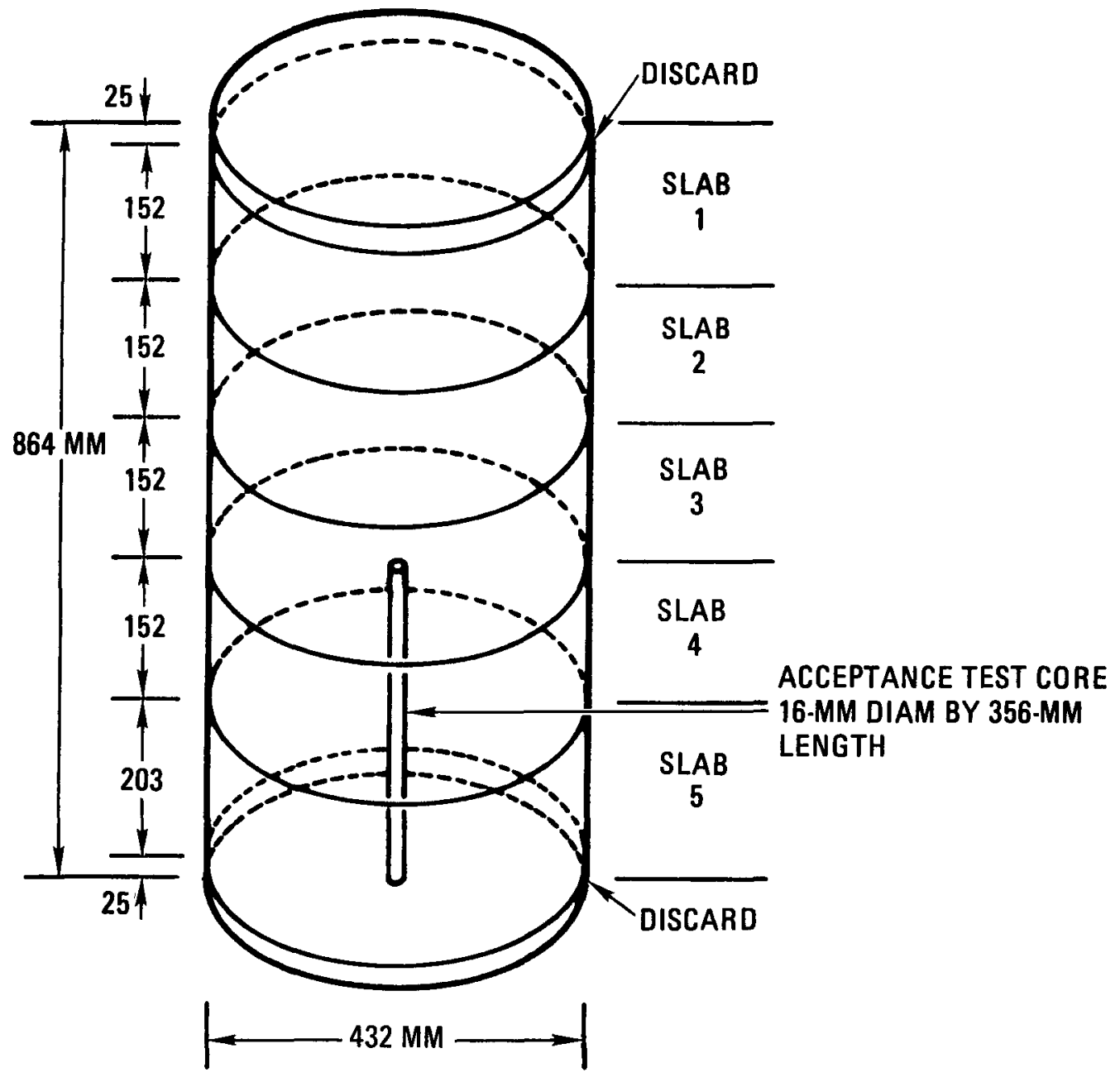

Fig. 1. Acceptance test core and characterization slabs for production grade $\mathrm{H}-451$ graphite 
in the characterization program while the acceptance test core penetrated slabs 4 and 5. In the acceptance testing (Ref. 7), the GLCC test specimen was taken from slab 5 material while the GA replicates were taken at the very end of the test core in slab 4, adjacent to slab 3. In previous characterizations of preproduction grade $\mathrm{H}-451$, slab 1 was designated at the same end as the acceptance test core (Refs. 3, 4). 


\section{EXPERIMENTAL METHODS}

Experimental methods used for determining bulk density, tensile propertles, thermal expansivity and conductivity, and chemical impurity content are given in Ref. 3. Poisson's ratio in tension was measured by the strain gage method of Ref. 3 using square cross section test specimens machined to retain identification of orthogonal material axes, as shown in Fig. 2 .

Compressive properties were measured in accordance with ASTM C695, using a wel1-characterized loading train for determination of modulus and stress-strain values. Flexural strengths were measured by four-point loading of $6.35-\mathrm{mm}$-diam, >51-mm-long solid cylinders. The outside load edges were spaced $38.1 \mathrm{~mm}$ apart. Flexural strength was calculated from modulus of rupture by accounting for the deviation from linear elasticity of the graphite. 

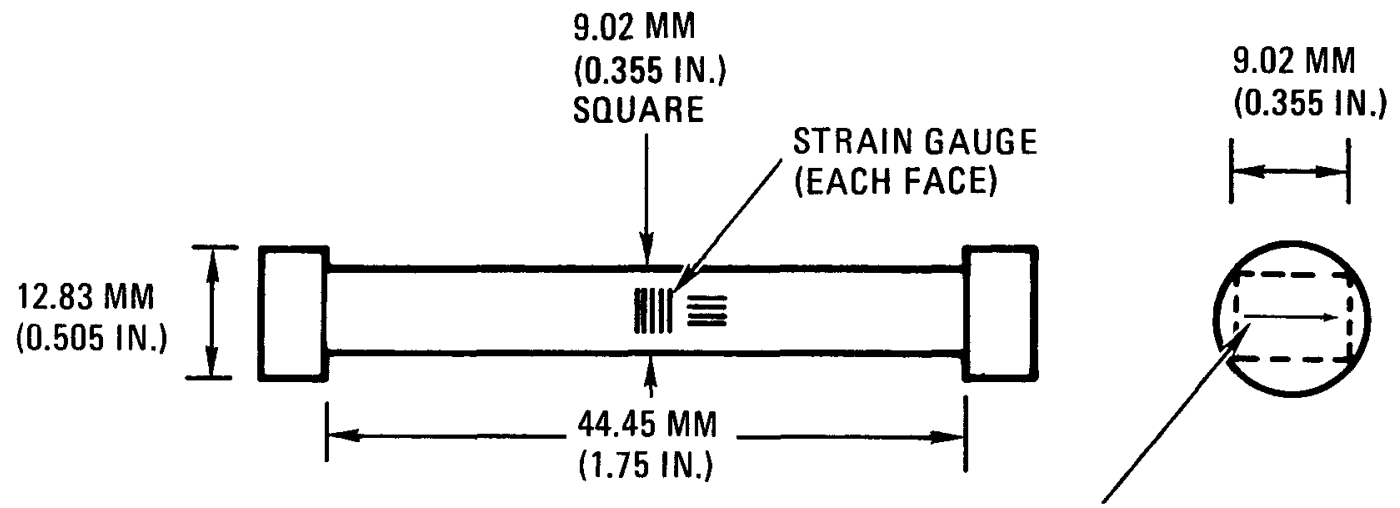

ARROW MARKED ON CORE

PARALLEL TO MATERIAL AXIS

Fig. 2. Poisson's ratio specimen geometry 


\section{EXPERIMENTAL RESULTS}

Data for individual specimens are given in Appendix $B$.

\subsection{BULK DENSITY}

The mean bulk density ranged from $1.724 \mathrm{Mg} / \mathrm{m}^{3}$ at the MLC of $10 \mathrm{~g}$ $7194-52$ to $1.757 \mathrm{Mg} / \mathrm{m}^{3}$ at the $\mathrm{EE}$ of $\log 7194-87$, as summarized in Table 3 . The average bulk density, measured over all three logs, was $1.740 \mathrm{Mg} / \mathrm{m}^{3}$, which compares well to the $1.74 \mathrm{Mg} / \mathrm{m}^{3}$ reported by GLCC (Table 2) and the mean of $1.736 \mathrm{Mg} / \mathrm{m}^{3}$ for all $98 \mathrm{H}-451$ production logs. The mean bulk density increased from center to edge of logs 7194-52 and -87. However, log 7194-49 displayed no density gradient from center to edge at the end of the $\log$.

The MLC position was a low-density region in the three logs tested. In $\log 7194-49$, the EC position was also a low-density region, whereas in the other two logs, the mean EC bulk density values were $0.010 \mathrm{Mg} / \mathrm{m}^{3}$ $(7194-52)$ and $0.016 \mathrm{Mg} / \mathrm{m}^{3}(7194-87)$ higher than the MLC densities.

Deviation in bulk density between logs $\left(S_{L L}\right)$ was approximately 0.010 $\mathrm{Mg} / \mathrm{m}^{3}$, while the pooled estimate of deviation within the sample from any given location in the $\log \left[\mathrm{S}_{\mathrm{WL}}\right.$ (pooled)] was $0.0045 \mathrm{Mg} / \mathrm{m}^{3}$.

\subsection{MECHANICAL PROPERTIES}

Results of tests for mechanical properties are included in the summary of property data, Table 3 . 
TABLE 3

SUMMARY OF PROPERTY DATA: H-451 LOT 478

\begin{tabular}{|c|c|c|c|c|c|c|c|c|c|c|c|c|c|c|c|c|c|c|c|}
\hline \multirow[b]{2}{*}{ Direction } & \multirow[b]{2}{*}{$\begin{array}{l}\text { Location } \\
\text { in Log } \\
\end{array}$} & \multirow[b]{2}{*}{$\begin{array}{l}\text { GA Iog } \\
\text { No. }\end{array}$} & \multicolumn{2}{|c|}{$\begin{array}{c}\text { Bulk Density } \\
\left(\mathrm{Mg} / \mathrm{m}^{3}\right)\end{array}$} & \multicolumn{2}{|c|}{$\begin{array}{c}\text { Tensile Strength } \\
(\mathrm{MPa})\end{array}$} & \multicolumn{2}{|c|}{$\begin{array}{c}\text { Tensile Modulus } \\
\text { (GPa) }\end{array}$} & \multicolumn{2}{|c|}{$\begin{array}{l}\text { Flexural Strength } \\
(\mathrm{MPa})\end{array}$} & \multicolumn{2}{|c|}{$\begin{array}{c}\text { Compressive Strength } \\
(\mathrm{MPa})\end{array}$} & \multicolumn{2}{|c|}{$\begin{array}{c}\text { Compressive Modulus } \\
\text { (GPa) }\end{array}$} & \multicolumn{2}{|c|}{$\begin{array}{c}\text { Coefficient of } \\
\text { Thermal Expansion } \\
(295-773 \mathrm{~K}) \\
\left(10^{-6} \mathrm{~K}^{-1}\right)\end{array}$} & \multicolumn{2}{|c|}{$\begin{array}{l}\text { Therma1 Conductivity } \\
(\mathrm{W} / \mathrm{m}-\mathrm{K})\end{array}$} & \multirow{2}{*}{$\begin{array}{c}\text { Ash } \\
\text { Content } \\
\text { (ppm) }\end{array}$} \\
\hline & & & $(\bar{X})$ & $\left(\mathrm{S}_{\mathrm{WL}} / \mathrm{N}\right)(\mathrm{a})$ & $(\bar{X})$ & $\left(\mathrm{S}_{\mathrm{WL}} / \mathrm{N}\right)^{(\mathrm{a})}$ & $(\overline{\mathrm{X}})$ & $\left(\mathrm{S}_{\mathrm{WL}} / \mathrm{N}\right)^{(\mathrm{a})}$ & $(\bar{X})$ & $\left(\mathrm{S}_{\mathrm{WL}} / \mathrm{N}\right)(\mathrm{a})$ & $(\overline{\mathrm{X}})$ & $\left(S_{W L} / N\right)(a)$ & $(\bar{x})$ & $\left(\mathrm{s}_{\mathrm{WL}} / \mathrm{N}\right)(\mathrm{a})$ & $(\overline{\mathrm{X}})$ & $\left(\mathrm{S}_{\mathrm{WL}} / \mathrm{N}\right)(\mathrm{a})$ & at $\begin{array}{c}1073 \mathrm{~K} \\
(\overline{\mathrm{X}})\end{array}$ & $\left(\mathrm{s}_{\mathrm{WL}} / \mathbb{N}\right)(\mathrm{a})$ & \\
\hline \multirow[t]{4}{*}{ Axial } & $\mathrm{EC}$ & $\begin{array}{r}7194-49 \\
-52 \\
-87\end{array}$ & $\begin{array}{l}1.735 \\
1.734 \\
1.751\end{array}$ & $\begin{array}{l}0.003 / 8 \\
0.002 / 8 \\
0.004 / 8\end{array}$ & $\begin{array}{l}13.4 \\
12.8 \\
13.9\end{array}$ & $\begin{array}{l}1.5 / 20 \\
1.5 / 20 \\
2.1 / 20\end{array}$ & $\begin{array}{l}8.5 \\
8.9 \\
9.1\end{array}$ & $\begin{array}{l}0.6 / 8 \\
0.2 / 8 \\
0.4 / 8\end{array}$ & $\begin{array}{l}20.7 \\
22.2 \\
24.2\end{array}$ & $\begin{array}{l}3.3 / 19 \\
2.8 / 20 \\
2.4 / 20\end{array}$ & $\begin{array}{l}49.2 \\
50.0 \\
53.6\end{array}$ & $\begin{array}{l}2.3 / 8 \\
2.3 / 8 \\
1.2 / 8\end{array}$ & $\begin{array}{l}6.2 \\
6.0 \\
6.9\end{array}$ & $\begin{array}{l}0.5 / 8 \\
0.4 / 8 \\
0.6 / 8\end{array}$ & 3.78 & $0.12 / 8$ & & & $\begin{array}{r}175 \\
88 \\
460\end{array}$ \\
\hline & $\mathrm{EE}$ & $\begin{array}{r}7194-49 \\
-52 \\
-87\end{array}$ & $\begin{array}{l}1.734 \\
1.741 \\
1.757\end{array}$ & $\begin{array}{l}0.009 / 8 \\
0.005 / 8 \\
0.003 / 8\end{array}$ & $\begin{array}{l}16.1 \\
18.8 \\
19.7\end{array}$ & $\begin{array}{l}2.2 / 20 \\
0.8 / 20 \\
1.1 / 20\end{array}$ & $\begin{array}{r}9.2 \\
10.4 \\
9.8\end{array}$ & $\begin{array}{l}0.5 / 8 \\
0.3 / 8 \\
0.3 / 8\end{array}$ & $\begin{array}{l}24.0 \\
27.2 \\
27.3\end{array}$ & $\begin{array}{l}2.0 / 20 \\
1.8 / 20 \\
1.6 / 20\end{array}$ & $\begin{array}{l}55.2 \\
58.3 \\
62.9\end{array}$ & $\begin{array}{l}4.0 / 8 \\
2.3 / 8 \\
2.3 / 8\end{array}$ & $\begin{array}{l}6.9 \\
6.8 \\
7.7\end{array}$ & $\begin{array}{l}0.4 / 8 \\
0.7 / 8 \\
0.4 / 8\end{array}$ & $\begin{array}{l}3.78 \\
3.99\end{array}$ & $\begin{array}{l}0.03 / 4 \\
0.30 / 8\end{array}$ & & & $\begin{array}{l}170 \\
132 \\
180\end{array}$ \\
\hline & MLC & $\begin{array}{r}7194-49 \\
-52 \\
-87\end{array}$ & $\begin{array}{l}1.736 \\
1.724 \\
1.735\end{array}$ & $\begin{array}{l}0.004 / 8 \\
0.004 / 8 \\
0.003 / 8\end{array}$ & $\begin{array}{l}14.1 \\
15.5 \\
15.6\end{array}$ & $\begin{array}{l}1.3 / 20 \\
0.9 / 20 \\
0.9 / 20\end{array}$ & $\begin{array}{l}8.8 \\
9.7 \\
9.2\end{array}$ & $\begin{array}{l}0.2 / 8 \\
0.2 / 8 \\
0.2 / 8\end{array}$ & $\begin{array}{l}22.0 \\
24.5 \\
24.0\end{array}$ & $\begin{array}{l}2.2 / 20 \\
1.8 / 20 \\
2.1 / 20\end{array}$ & $\begin{array}{l}50.3 \\
51.8 \\
53.7\end{array}$ & $\begin{array}{l}2.3 / 8 \\
2.4 / 8 \\
1.0 / 8\end{array}$ & $\begin{array}{l}6.3 \\
6.4 \\
6.6\end{array}$ & $\begin{array}{l}0.2 / 7 \\
0.4 / 8 \\
0.5 / 8\end{array}$ & $\begin{array}{l}3.72 \\
3.62\end{array}$ & $\begin{array}{l}0.08 / 4 \\
0.15 / 8\end{array}$ & 62.8 & $5.8 / 8$ & $\begin{array}{r}205 \\
96 \\
660\end{array}$ \\
\hline & MLE & $\begin{array}{r}7194-49 \\
-52 \\
-87 \\
\end{array}$ & $\begin{array}{l}1.743 \\
1.739 \\
1.749 \\
\end{array}$ & $\begin{array}{l}0.006 / 8 \\
0.004 / 8 \\
0.004 / 8 \\
\end{array}$ & $\begin{array}{l}18.6 \\
18.6 \\
20.0 \\
\end{array}$ & $\begin{array}{l}1.3 / 20 \\
1.5 / 20 \\
0.9 / 20 \\
\end{array}$ & $\begin{array}{r}9.8 \\
10.3 \\
9.9 \\
\end{array}$ & $\begin{array}{l}0.4 / 8 \\
0.3 / 8 \\
0.2 / 8 \\
\end{array}$ & $\begin{array}{l}27.4 \\
27.7 \\
28.4 \\
\end{array}$ & $\begin{array}{l}1.6 / 20 \\
1.9 / 20 \\
1.3 / 20 \\
\end{array}$ & $\begin{array}{l}58.9 \\
58.9 \\
60.5 \\
\end{array}$ & $\begin{array}{l}1.8 / 8 \\
1.5 / 8 \\
1.3 / 8 \\
\end{array}$ & $\begin{array}{l}6.8 \\
6.7 \\
7.3 \\
\end{array}$ & $\begin{array}{l}0.5 / 8 \\
0.3 / 8 \\
0.2 / 8 \\
\end{array}$ & 3.73 & $0.07 / 8$ & 65.0 & $3.5 / 8$ & $\begin{array}{l}147 \\
160 \\
255 \\
\end{array}$ \\
\hline \multirow[t]{4}{*}{ Radial } & $\mathrm{EC}$ & $\begin{array}{r}7194-49 \\
-52 \\
-87\end{array}$ & $\begin{array}{l}1.737 \\
1.732 \\
1.748\end{array}$ & $\begin{array}{l}0.003 / 8 \\
0.003 / 8 \\
0.003 / 8\end{array}$ & $\begin{array}{l}12.4 \\
11.9 \\
12.6\end{array}$ & $\begin{array}{l}1.4 / 20 \\
2.6 / 20 \\
2.1 / 20\end{array}$ & $\begin{array}{l}7.8 \\
8.2 \\
7.9\end{array}$ & $\begin{array}{l}0.2 / 8 \\
0.4 / 8 \\
0.3 / 8\end{array}$ & $\begin{array}{l}21.4 \\
22.2 \\
24.1\end{array}$ & $\begin{array}{l}1.9 / 20 \\
2.3 / 20 \\
1.8 / 20\end{array}$ & $\begin{array}{l}49.4 \\
52.2 \\
53.1\end{array}$ & $\begin{array}{l}1.9 / 8 \\
2.1 / 8 \\
2.8 / 8\end{array}$ & $\begin{array}{l}5.4 \\
5.4 \\
5.7\end{array}$ & $\begin{array}{l}0.3 / 8 \\
0.2 / 8 \\
0.5 / 8\end{array}$ & 4.46 & $0.10 / 8$ & & & \\
\hline & $\mathrm{EE}$ & $\begin{array}{r}7194-49 \\
-52 \\
-87\end{array}$ & $\begin{array}{l}1.735 \\
1.740 \\
1.756\end{array}$ & $\begin{array}{l}0.006 / 8 \\
0.003 / 8 \\
0.003 / 8\end{array}$ & $\begin{array}{l}15.5 \\
15.9 \\
16.7\end{array}$ & $\begin{array}{l}1.5 / 20 \\
2.4 / 20 \\
1.4 / 20\end{array}$ & $\begin{array}{l}8.0 \\
8.8 \\
8.5\end{array}$ & $\begin{array}{l}0.2 / 8 \\
0.2 / 8 \\
0.3 / 8\end{array}$ & $\begin{array}{l}23.7 \\
25.1 \\
26.1\end{array}$ & $\begin{array}{l}1.5 / 20 \\
1.7 / 20 \\
1.1 / 20\end{array}$ & $\begin{array}{l}55.9 \\
57.8 \\
56.1\end{array}$ & $\begin{array}{l}1.9 / 8 \\
1.7 / 8 \\
2.0 / 8\end{array}$ & $\begin{array}{l}5.7 \\
5.5 \\
6.1\end{array}$ & $\begin{array}{l}0.4 / 8 \\
0.2 / 8 \\
0.4 / 8\end{array}$ & $\begin{array}{l}4.52 \\
4.70\end{array}$ & $\begin{array}{l}0.07 / 4 \\
0.19 / 8\end{array}$ & & & \\
\hline & MLC & $\begin{array}{r}7194-49 \\
-52 \\
-87\end{array}$ & $\begin{array}{l}1.736 \\
1.725 \\
1.733\end{array}$ & $\begin{array}{l}0.004 / 8 \\
0.003 / 8 \\
0.003 / 8\end{array}$ & $\begin{array}{l}11.7 \\
10.7 \\
10.9\end{array}$ & $\begin{array}{l}1.6 / 20 \\
2.5 / 20 \\
1.2 / 20\end{array}$ & $\begin{array}{l}7.7 \\
7.8 \\
7.4\end{array}$ & $\begin{array}{l}0.2 / 8 \\
0.6 / 8 \\
0.3 / 8\end{array}$ & $\begin{array}{l}19.2 \\
18.1 \\
18.9\end{array}$ & $\begin{array}{l}1.8 / 20 \\
3.4 / 20 \\
1.9 / 20\end{array}$ & $\begin{array}{l}52.4 \\
48.3 \\
48.2\end{array}$ & $\begin{array}{l}0.9 / 8 \\
1.0 / 8 \\
1.7 / 8\end{array}$ & $\begin{array}{l}5.2 \\
4.7 \\
5.0\end{array}$ & $\begin{array}{l}0.2 / 8 \\
0.3 / 8 \\
0.5 / 8\end{array}$ & $\begin{array}{l}4.27 \\
4.35\end{array}$ & $\begin{array}{l}0.10 / 4 \\
0.12 / 8\end{array}$ & $\begin{array}{l}60.2 \\
58.3\end{array}$ & $\begin{array}{l}6.0 / 5 \\
4.9 / 8\end{array}$ & \\
\hline & MLE & $\begin{array}{r}7194-49 \\
-52 \\
-87\end{array}$ & $\begin{array}{l}1.743 \\
1.739 \\
1.748\end{array}$ & $\begin{array}{l}0.006 / 8 \\
0.005 / 8 \\
0.002 / 8\end{array}$ & $\begin{array}{l}14.6 \\
14.3 \\
15.0\end{array}$ & $\begin{array}{l}1.3 / 20 \\
2.6 / 20 \\
1.4 / 20\end{array}$ & $\begin{array}{l}8.1 \\
8.8 \\
8.3\end{array}$ & $\begin{array}{l}0.2 / 8 \\
0.3 / 7 \\
0.1 / 8\end{array}$ & $\begin{array}{l}22.2 \\
24.2 \\
24.0\end{array}$ & $\begin{array}{l}1.9 / 20 \\
1.2 / 20 \\
1.2 / 20\end{array}$ & $\begin{array}{l}54.6 \\
54.5 \\
50.3\end{array}$ & $\begin{array}{l}1.8 / 8 \\
1.6 / 8 \\
1.7 / 8\end{array}$ & $\begin{array}{l}5.4 \\
5.2 \\
5.4\end{array}$ & $\begin{array}{l}0.3 / 8 \\
0.3 / 8 \\
0.3 / 8\end{array}$ & 4.32 & $0.31 / 8$ & $\begin{array}{l}59.4 \\
60.7\end{array}$ & $\begin{array}{l}5.3 / 8 \\
2.5 / 8\end{array}$ & \\
\hline
\end{tabular}

\footnotetext{
$(a)_{N}=$ number of replicate specimens.
} 
$$
\text { . }
$$ 


\subsubsection{Strength}

Tensile strengths were highest at the edge of the logs. Lowest mean strengths were observed at the MLC for the radial orientation, but mean axial tensile strength was lowest at the EC. Mean axial UTS at EC ranged from $12.8 \mathrm{MPa}$ in $\log 7194-52$ to $13.9 \mathrm{MPa}$ for $\log 7194-87$. Maximum mean axial UTS occurred at the MLE of the logs, ranging from 18.6 to $20.0 \mathrm{MPa}$ over the three logs. The radial UTS averaged $10.7 \mathrm{MPa}$ in $\log 7194-52$ to $11.7 \mathrm{MPa}$ in $\log 7194-49$ at MLC. Highest radial UTS values occurred at $\mathrm{EE}$, averaging 15.5 to $16.7 \mathrm{MPa}$.

$\mathrm{S}_{\mathrm{WL}}$ (pooled) was $1.44 \mathrm{MPa}$ for the axial direction and $1.96 \mathrm{MPa}$ in the radial orientation. $\mathrm{S}_{\mathrm{LL}}$ (pooled) in UTS was $1.39 \mathrm{MPa}$ axially and $0.58 \mathrm{MPa}$ radially.

Log 7194-52 was sampled at both ends (slabs 1 and 5) for tensile strength, and both ends displayed lower axial UTS than the MLC. The distribution of UTS in $\log 7192-52$ is given in Table 4.

Flexural strength and ultimate compressive strength (UCS) followed the same spatial relationships observed in tensile strength. Figure 3 presents the rank of each log position for UTS, flexural strength, UCS, and density for axial and radial orientations.

Mean flexural strengths in the axial direction ranged from $20.7 \mathrm{MPa}$ at EC of lot $7194-49$ to $28.4 \mathrm{MPa}$ at MLE of $\log 7194-87$. Mean radial flexural strength varied from $18.1 \mathrm{MPa}$ (MLC, 7194-52) to $26.1 \mathrm{MPa}$ (EE, 7194-87). UCS averaged from $48.2 \mathrm{MPa}$ (MLC, radia1) to $62.9 \mathrm{MPa}$ (EE, axial). The deviations in flexural strength were $\mathrm{S}_{\mathrm{WL}}$ (pooled) = $2.18 \mathrm{MPa}$ axially and $1.95 \mathrm{MPa}$ radially; $\mathrm{S}_{L L}$ (pooled) $=1.80 \mathrm{MPa}$ axially and $1.36 \mathrm{MPa}$ radia11y. UCS deviations were estimated to be 2.35 and $1.95 \mathrm{MPa}$ for $\mathrm{S}_{\mathrm{WL}}$ (pooled) and 3.02 and $2.49 \mathrm{MPa}$ for $\mathrm{S}_{\mathrm{LL}}$ (pooled) in the axial and radial directions, respectively. 
TABLE 4

DISTRIBUTION OF TENSILE STRENGTH IN

LOG 7194-52

\begin{tabular}{c|c|c|c|c}
\hline \multirow{2}{*}{} & \multicolumn{2}{|c|}{ Center Location } & \multicolumn{2}{c}{ Edge Location } \\
\cline { 2 - 5 } S1ab & \multicolumn{2}{|c|}{ UTS (MPa) } & \multicolumn{2}{|c}{ UTS (MPa) } \\
\cline { 2 - 5 } & Axial & Radial & Axial & Radial \\
\hline & & & & \\
3 & 12.8 & 11.9 & 18.8 & 15.9 \\
5 & 15.5 & 10.7 & 18.6 & 14.3 \\
& 13.8 & 12.0 & 19.1 & 13.5 \\
\hline
\end{tabular}




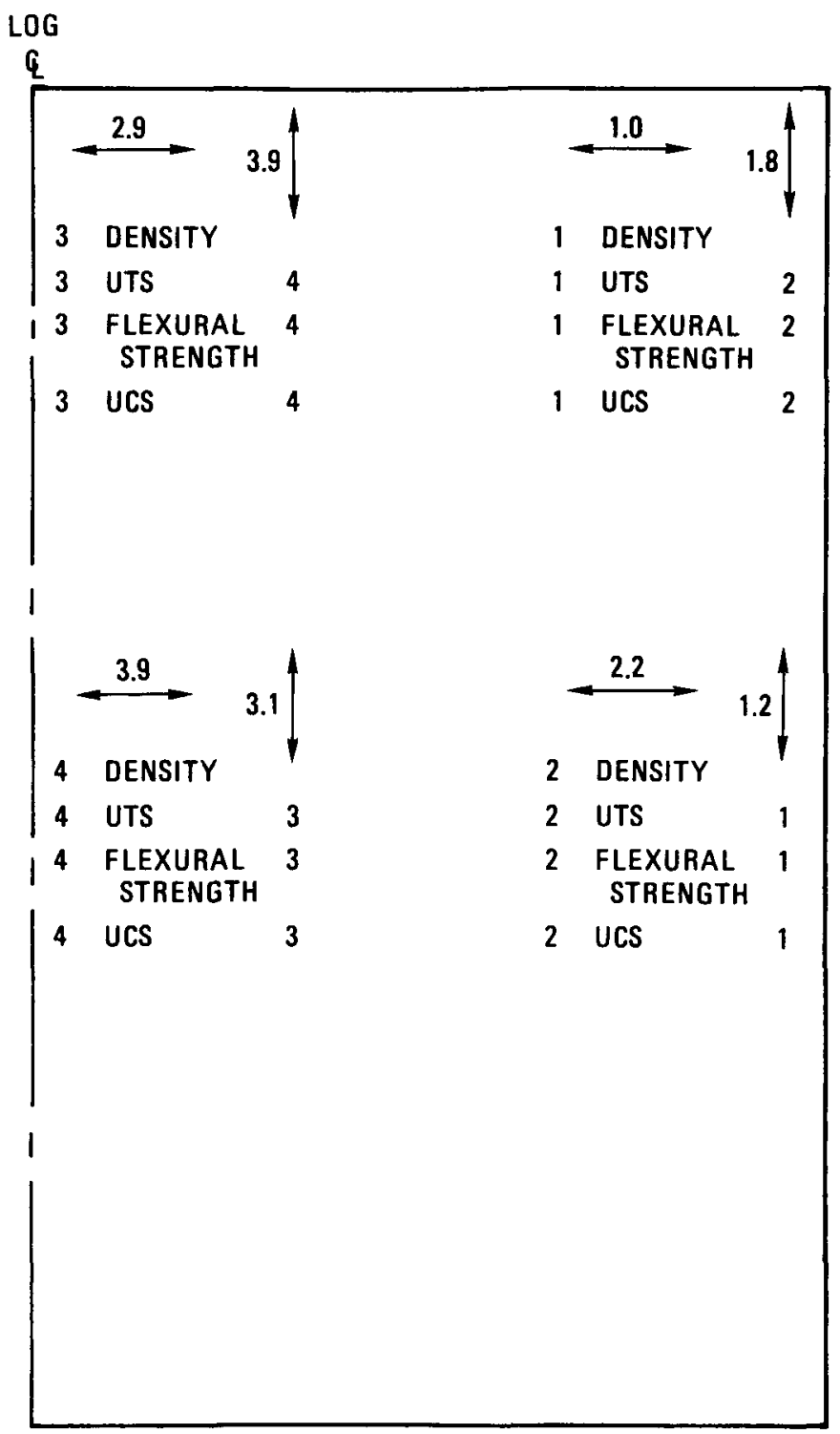

LEGEND
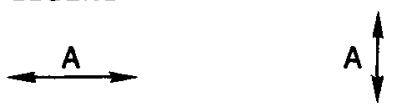

B PROPERTY B

$A=$ AVERAGE OF UTS RANK FOR THREE LOGS IN DIRECTION INDICATED ( $\longrightarrow=$ RADIAL, $\downarrow=$ AXIAL).

$B=$ RANK OF THREE LOG AVERAGE FOR PROPERTY GIVEN AND ORIENTATION INDICATED AT TOP OF COLUMN. RANK: HIGHEST MEAN VALUE $=1$, LOWEST MEAN VALUE $=4$.

Fig. 3. Rank of properties versus location in $10 g$ 
Flexural strength averaged 53\% higher than UTS in the axial direction and $67 \%$ higher radially. The ratio of UCS to UTS was 3.4 axially and 4.0 radially.

The greatest anisotropy in strength between axial and radial directions was observed at MLC where axial UTS was $65 \%$ higher than radial UTS. Anisotropy in tensile strengths varied from $5 \%$ to $20 \%$ at the ends of the $\log$ and averaged $30 \%$ at MLE.

\subsubsection{Elastic Modulus}

The elastic modulus in tension was measured as a chord modulus between 0 and $6.9 \mathrm{MPa}$ during the second loading of the test specimens. Compressive modulus was determined in a like manner, but over the stress range of 0 to $27.6 \mathrm{MPa}$. The elastic modulus in tension averaged 8.5 to $10.4 \mathrm{GPa}$ in the axial specimens and 7.4 to $8.8 \mathrm{GPa}$ in the radial specimens. Compressive modulus values were somewhat lower due to the larger deviation from linear elasticity at high stress levels. Modulus values in compression varled from 6.0 to $7.7 \mathrm{GPa}$ axially and from 4.7 to $6.1 \mathrm{GPa}$ radially.

$\mathrm{S}_{\mathrm{WL}}$ (pooled) was approximately $0.4 \mathrm{GPa}$ for both tensile and compressive moduli. $\mathrm{S}_{\mathrm{LL}}$ (pooled) was $0.3 \mathrm{GPa}$ for compressive modulus and $0.4 \mathrm{GPa}$ for tensile modulus.

\subsubsection{Poisson's Ratio}

Poisson's ratios $\left(\nu_{1 j}\right)$ were measured from H-451 graphite $\log 7194-52$, slab 1, EE. $\nu_{i j}$ is reported as the negative ratio of strain along the $j$ axis to strain along the $i$ axis when stress is applied along the $i$ axis. In this work, the radial direction was taken as axis No. 1, the chord perpendicular to the $10 \mathrm{~g}$ radius as direction No. 2 , and the cylindrical axis of the graphite $\log$ as axis No. 3. Poisson's ratios were measured for specimens stressed along axes 1 and 3 . Results are summarized in Table 5. Poisson's ratios of $v_{13}=0.117, v_{12}=0.150$, and $v_{31}=v_{32}$ $=0.130$ were measured for $\mathrm{H}-451$ graphite. 
TABLE 5

POISSON'S RATIOS IN H-451 GRAPHITE

\begin{tabular}{|c|c|c|c|c|c|c|c|}
\hline Log & Location & Specimen & Gauge & $v_{12}$ & $v_{13}$ & $v_{31}$ & $\nu_{32}$ \\
\hline $7194-52$ & $\mathrm{EE}$ & $\begin{array}{l}\text { P1 } \\
\text { P2 } \\
\text { P3 } \\
\text { P4 }\end{array}$ & $\begin{array}{l}\text { TG100 } \\
\text { TM62 } \\
\text { TG100 } \\
\text { TM62 } \\
\text { TG100 } \\
\text { TM62 } \\
\text { TG100 } \\
\text { TM62 }\end{array}$ & $\begin{array}{l}0.140 \\
0.079 \\
0.153 \\
0.146\end{array}$ & $\begin{array}{l}0.117 \\
0.064 \\
0.118 \\
0.112\end{array}$ & $\begin{array}{l}0.140 \\
0.113 \\
0.123 \\
0.130\end{array}$ & $\begin{array}{l}0.132 \\
0.102 \\
0.139 \\
0.140\end{array}$ \\
\hline
\end{tabular}




\subsection{THERMAL PROPERTIES}

Thermal properties are summarized In Table 3.

\subsubsection{Thermal Expansivity}

The mean coefficient of thermal expansion between room temperature and $773 \mathrm{~K}\left(\bar{\alpha}_{295-773 \mathrm{~K}}\right)$ ranged from 3.62 to $3.99 \times 10^{-6} \mathrm{~K}^{-1}$ in the axial direction. In the radial direction, $\bar{\alpha}_{295-773 \mathrm{~K}}$ varied from 4.27 to 4.70 $x 10^{-6} \mathrm{~K}^{-1}$ with the highest expansivity occurring at $\mathrm{EE}$, as in the axial direction. The lowest mean values of $\bar{\alpha}_{295-773 \mathrm{~K}}$ were found at MLC for both axial and radial directions. The anisotropy ratio of $\bar{\alpha}_{295-773 \mathrm{~K}}$ (radial)/ $\bar{\alpha}_{295-773 \mathrm{~K}}$ (axial) was 1.18 at the end of $\log 7194-52,1.20$ at MLC, and 1.16 at MLE. Anisotropy ratios in $\log 7194-49$ were 1.20 at EE and 1.15 at MLC.

\subsubsection{Thermal Conductivity}

Thermal conductivity versus measurement temperature is given in Table 6. Conductivity was measured at the MLC and MLE of 1ogs 7194-52 and 7194-49. A slight anisotropy in conductivity was noted with axial specimens averaging $7 \%$ higher conductivity than the radial specimens. At a measurement temperature of $1073 \mathrm{~K}$, axial conductivity averaged 62.8 and $65.0 \mathrm{~W} / \mathrm{m}-\mathrm{K}$ and radial thermal conductivity 59.3 and $60.0 \mathrm{~W} / \mathrm{m}-\mathrm{K}$ at MLC and MLE, respective1y.

\subsection{CHEMICAL PURITY}

The principal impurity elements and their concentrations in production grade $\mathrm{H}-451$ are listed in Table 7 . Total ash content ranged from $90 \mathrm{ppm}$ in the center of $\log 7194-52$ to $660 \mathrm{ppm}$ at MLC of $\log 7194-87$. Boron content, as measured by emission spectroscopy, varied from 2 to $4 \mathrm{ppm}$ in all logs. The iron impurity level was lowest ( 5 to $15 \mathrm{ppm}$ ) in the high-ash $\log 7194-87$ and highest (40 to $60 \mathrm{ppm}$ ) in $\log 7194-49$ whose ash content was $\sim 180 \mathrm{ppm}$. 
TABLE 6

THERMAL CONDUCTIVITY OF H-451 GRAPHITE

\begin{tabular}{|c|c|c|c|c|c|c|c|}
\hline \multirow[b]{2}{*}{ Log } & \multirow[b]{2}{*}{ Location } & \multirow[b]{2}{*}{ Orientation } & \multicolumn{5}{|c|}{$\begin{array}{l}\text { Thermal Conductivity } \\
(\mathrm{W} / \mathrm{m}-\mathrm{K})\end{array}$} \\
\hline & & & $295 \mathrm{~K}$ & $473 K$ & $673 K$ & $873 \mathrm{~K}$ & $1073 \mathrm{~K}$ \\
\hline \multirow[t]{4}{*}{$7194-52$} & MLC & Axial & 134.0 & 116.8 & 93.6 & 75.4 & 62.8 \\
\hline & & Radial & 119.6 & 102.7 & 81.2 & 68.0 & 58.3 \\
\hline & MLE & Axial & 132.9 & 117.3 & 95.1 & 75.2 & 65.0 \\
\hline & & Radial & 124.8 & 107.4 & 85.0 & 69.8 & 60.7 \\
\hline \multirow[t]{2}{*}{$7194-49$} & MLC & Radial & 116.5 & 103.6 & 82.4 & 66.2 & 60.2 \\
\hline & MLE & Radial & 122.8 & 106.4 & 86.2 & 68.3 & 59.4 \\
\hline
\end{tabular}


TABLE 7

H-451 IMPURITY CONTENT, LOT 478

\begin{tabular}{|c|c|c|c|c|c|c|c|c|c|c|c|}
\hline \multirow[b]{2}{*}{$\log$} & \multirow[b]{2}{*}{ Location } & \multicolumn{9}{|c|}{$\begin{array}{c}\text { Impurity Content } \\
(\mathrm{ppm})\end{array}$} & \multirow{2}{*}{$\begin{array}{c}\text { Nonburnable } \\
\text { Boron Equivalent } \\
\text { (ppm) }\end{array}$} \\
\hline & & Ash & B & $\mathrm{Fe}$ & $\mathrm{V}$ & $\mathrm{Ti}$ & $\mathrm{Ni}$ & $\mathrm{A} 1$ & $\mathrm{Si}$ & $\mathrm{Mg}$ & \\
\hline \multirow[t]{4}{*}{$7194-49$} & EC & 175 & 2 & 40 & 6 & 6 & 10 & 10 & 60 & 2 & 0.08 \\
\hline & $\mathrm{EE}$ & 170 & 3 & 40 & 6 & 6 & 10 & 10 & 90 & 2 & 0.08 \\
\hline & MLC & 205 & 4 & 60 & 60 & 40 & $<4$ & 30 & 60 & 15 & 0.23 \\
\hline & MLE & 147 & 4 & 60 & 15 & 15 & $<4$ & 30 & 80 & 30 & 0.13 \\
\hline \multirow[t]{4}{*}{$7194-52$} & EC & 88 & 4 & 20 & 20 & 10 & $<4$ & 20 & 60 & 10 & 0.08 \\
\hline & $\mathrm{EE}$ & 132 & 4 & 40 & 2 & 2 & $<4$ & 10 & 80 & 4 & 0.06 \\
\hline & MLC & 97 & 3 & 10 & 4 & 4 & $<4$ & 5 & 70 & 4 & 0.05 \\
\hline & MLE & 160 & 3 & 10 & 4 & 4 & $<4$ & 4 & 70 & 4 & 0.05 \\
\hline \multirow[t]{4}{*}{$7194-87$} & EC & 460 & 2 & 15 & 30 & 30 & $<4$ & $<1$ & 60 & 3 & 0.13 \\
\hline & $E E$ & 180 & 2 & 10 & 14 & 24 & $<4$ & $<1$ & 70 & 4 & 0.10 \\
\hline & MLC & 660 & 2 & 10 & 80 & 60 & $<4$ & $<1$ & 80 & 6 & 0.25 \\
\hline & MLE & 255 & 2 & 5 & 45 & 35 & $<4$ & $<1$ & 80 & 4 & 0.15 \\
\hline
\end{tabular}


The high ash content graphite appeared to contain high levels of vanadium, titanium, and sllicon, as given in Table 7. The only other elements detected by emission spectroscopy were occasional reports of potassium (10 ppm), copper (10-20 ppm), and lithium (1 ppm). However, due to uncertainties in this method, the lithium values are suspect.

The boron equivalent of nonburnable elements detectable by GA standard emission spectroscopy methods ranged between 0.05 and $0.25 \mathrm{ppm}$ for a11 three graphite logs. Certain other elements, mainly burnable elements, have relatively high limits of detection with this method and so contribute to the total calculated boron equivalence of the graphite. However, more sensitive analyses done in past H-451 samples indicate that most elements not detected by standard emission spectroscopy are either present in very low concentrations, or have very low boron equivalence factors, and therefore contribute little to the actual nonburnable boron equivalent. 


\section{DISCUSSION AND CONCLUSIONS}

The three logs characterized displayed property values typical of near-isotropic fuel element graphite. The bulk density, UTS, flexural strength, UCS, thermal conductivity, and thermal expansivity values were comparable to the values measured in preproduction grade H-451, 1ot 426 (Ref. 4). As shown in Table 8, the bulk density and UTS at MLC were slightly higher in the production grade (lot 478) $\mathrm{H}-451$. Although elastic modulus values were slightly higher than in preproduction $\mathrm{H}-451$, their effect will be tempered by the higher strengths and lower thermal expansivities reported in lot 478 . Thermal conductivity remained virtually unchanged from preproduction to production material.

Impurity levels in three production grade $\mathrm{H}-451$ logs were noticeably higher than observed in lot 426 (Table 8); however, a11 major impurities were within specified limits. No relationship between ash content and iron content could be established. High ash graphite apparently consisted principally of silicon, vanadium, and titanium impurities. Analysis for the most commonly occurring impurity elements in graphite by spark mass spectrometry detected impurity element concentrations equivalent to 0.05 to $0.25 \mathrm{ppm}$ boron for nonburnable impurities.

The axial tensile strength of the production $\mathrm{H}-451$ was lower at EC than at MLC of the logs. Previous data on H-451 graphite logs (Refs. 1,3 , and 4) showed MLC to be the weakest location.

The production H-451 graphite is as good or better product than the preproduction precursors of $\mathrm{H}-451$. The property values derived from tests on preproduction $\mathrm{H}-451$ graphite remain valid within $\pm 5 \%$ error for most properties of production grade $\mathrm{H}-451$ graphite. The characterization data reported above, when combined with preproduction characterization data, provide a consistent data base for design with unirradiated $\mathrm{H}-451$ graphite. 
TABLE 8

COMPARISON OF PROPERTIES OF PRODUCTION AND PREPRODUCTION H-451 GRAPHITE

\begin{tabular}{|c|c|c|c|c|c|c|c|c|c|c|c|}
\hline \multirow[b]{2}{*}{ Lot } & \multirow[b]{2}{*}{ Log } & \multirow[b]{2}{*}{$\begin{array}{c}\text { Bu1k Density } \\
\left(\mathrm{Mg} / \mathrm{m}^{3}\right)\end{array}$} & \multicolumn{2}{|c|}{ UTS } & \multirow{2}{*}{$\begin{array}{c}\text { Modulus } \\
\text { Axial MLC } \\
(\mathrm{GPa}) \\
\end{array}$} & \multicolumn{2}{|c|}{${ }^{\bar{\alpha}}(295-773 \mathrm{~K})$} & \multicolumn{2}{|c|}{$\begin{array}{c}\text { Thermal Conductivity } \\
\text { at } 1073 \mathrm{~K}\end{array}$} & \multirow[b]{2}{*}{$\begin{array}{c}\text { Ash } \\
(\mathrm{ppm})\end{array}$} & \multirow[b]{2}{*}{$\begin{array}{l}\text { Iron } \\
(\mathrm{ppm})\end{array}$} \\
\hline & & & $\begin{array}{c}\text { Axial MLC } \\
(\mathrm{MPa})\end{array}$ & $\begin{array}{c}\text { Radial MLC } \\
(\mathrm{MPa})\end{array}$ & & $\begin{array}{l}\text { Axial MLC } \\
\left(10^{-6} K^{-1}\right) \\
\end{array}$ & $\begin{array}{l}\text { Radial MLC } \\
(10-6 K-1)\end{array}$ & $\begin{array}{c}\text { Axial MLC } \\
(\mathrm{W} / \mathrm{m}-\mathrm{K})\end{array}$ & $\begin{array}{c}\text { Radial MLC } \\
(\mathrm{W} / \mathrm{m}-\mathrm{K})\end{array}$ & & \\
\hline 478 & $7194-49$ & 1.738 & 14.1 & 11.7 & 8.8 & 3.72 & 4.27 & -- & 60.2 & 175 & 50 \\
\hline \multirow[t]{2}{*}{ (Production) } & $7194-52$ & 1.735 & 15.5 & 10.7 & 9.7 & 3.62 & 4.35 & 62.8 & 58.3 & 120 & 20 \\
\hline & $7194-87$ & 1.748 & 15.6 & 10.9 & 9.2 & -- & -- & -- & -- & 400 & 10 \\
\hline \multicolumn{12}{|l|}{426} \\
\hline \multirow[t]{4}{*}{ (Preproduction) } & $6484-33$ & 1.739 & 14.5 & 10.4 & 8.1 & 4.08 & 4.51 & -- & -- & $<25$ & 3.5 \\
\hline & $6484-34$ & 1.719 & 14.2 & 10.4 & 8.2 & 4.10 & 4.63 & 64.9 & 62.8 & $<25$ & 7.5 \\
\hline & $6484-40$ & 1.720 & 13.0 & 11.7 & 7.9 & -- & -- & -- & -- & 89 & $<1$ \\
\hline & $6484-41$ & 1.707 & 12.9 & 10.4 & 7.6 & 3.92 & 4.42 & 64.4 & 62.8 & 41 & $<1$ \\
\hline
\end{tabular}




\section{ACKNOWLEDGMENTS}

The author gratefully acknowledges the guidance and many helpful discussions of G. B. Engle and Dr. R. J. Price. Thanks are extended to

C. H. Richards, J. R. Whaley, F. Hogan, M. G. Dunlap, G. L. Campbell, C. Chaney, M. Denny, and W. Baugh for their able assistance and excellent laboratory work. 
1. Engle, G. B., "Assessment of Grade H-451 Graphite for Replaceable Fuel and Reflector Elements in HTGR," DOE Report GA-A14690, General Atomic Company, December 1977.

2. Price, R. J., and L. A. Beavan, "Final Report on Graphite Irradiation Test OG-1," USAEC Report GA-A13089, General Atomic Company, August 1, 1974.

3. Johnson, W. R., and G. B. Engle, "Properties of Unirradiated Fuel Element Graphites H-451 and TS1240," ERDA Report GA-A13752, General Atomic Company, January 31, 1976.

4. Engle, G. B., and W. R. Johnson, "Properties of Unirradiated Fuel Element Graphites H-451 and S0818," ERDA Report GA-A14068, General Atomic Company, October 8, 1976.

5. Price, R. J., and L. A. Beavan, "Final Report on Graphite Irradiation Test OG-2," ERDA Report GA-A13556, General Atomic Company, December 15, 1975.

6. Price, R. J., and L. A. Beavan, "Final Report on Graphite Irradiation Test OG-3," ERDA Report GA-A14211, General Atomic Company, January 1977.

7. Engle, G. B., and R. J. Price, "Strength Testing of Production Grade H-451 Graphite; Lots 472, 478, and 482," ERDA Report GA-A14269, General Atomic Company, March 1977. 
APPENDIX A

SAMPLING PLAN - MOT-007 
0

- 


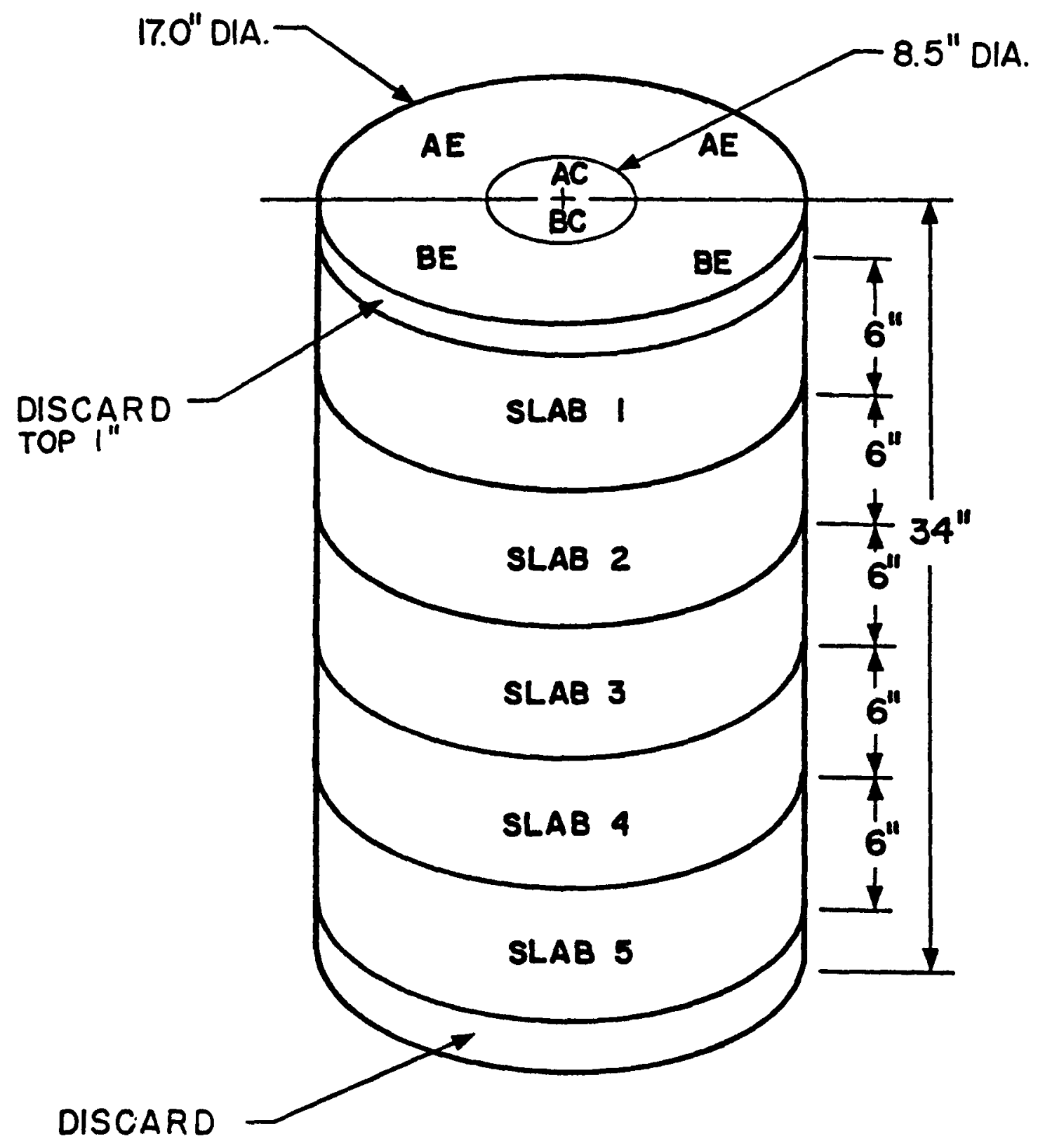

F1g. A-1. Slab diagram - replaceable hexagonal fuel element and reflector blocks: graphite log $\mathrm{H}-451$ (sheet 1 of 5) 


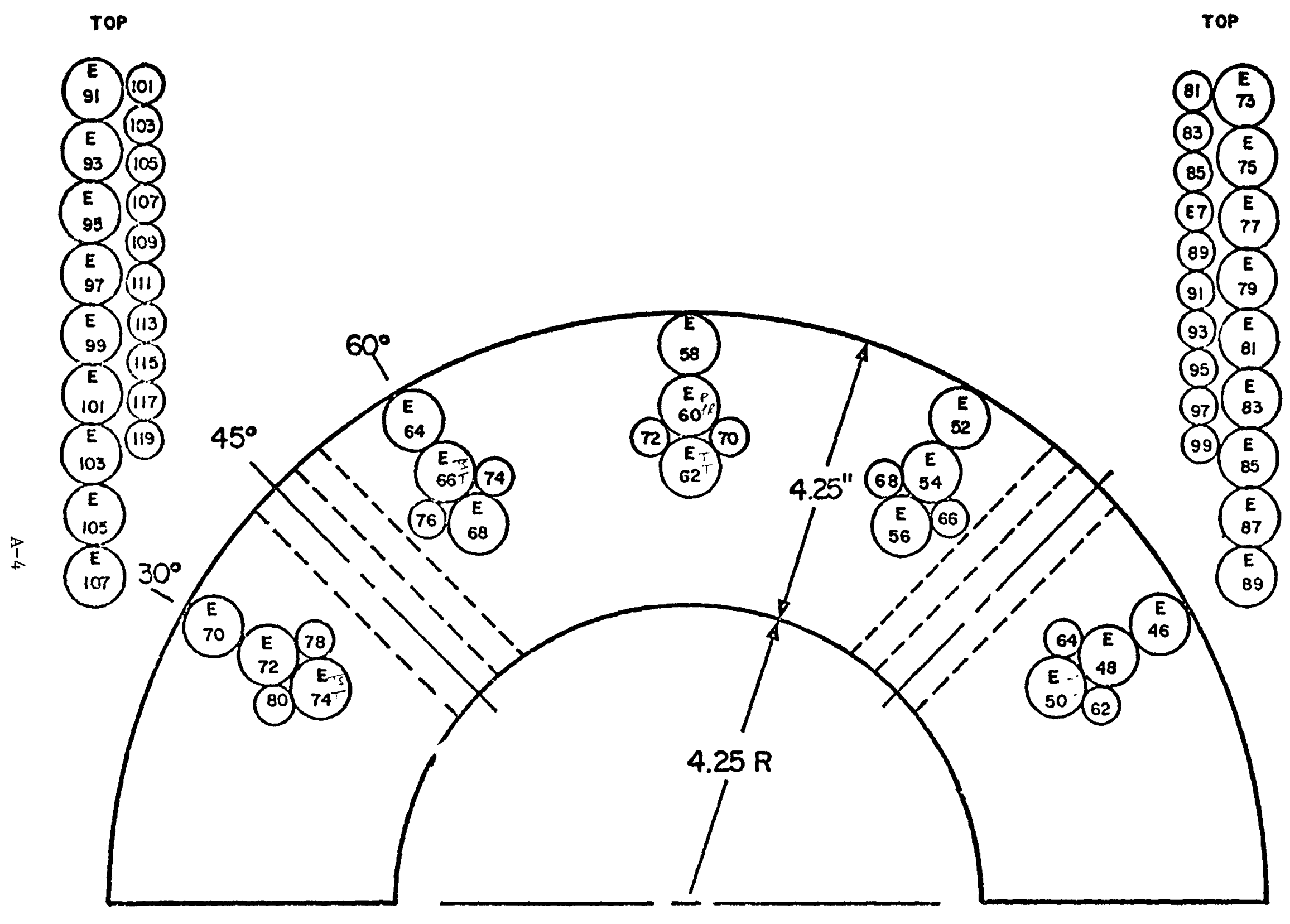

Fig. A-1. Edge Section $1 \mathrm{AE}$ (sheet 2 of 5) 


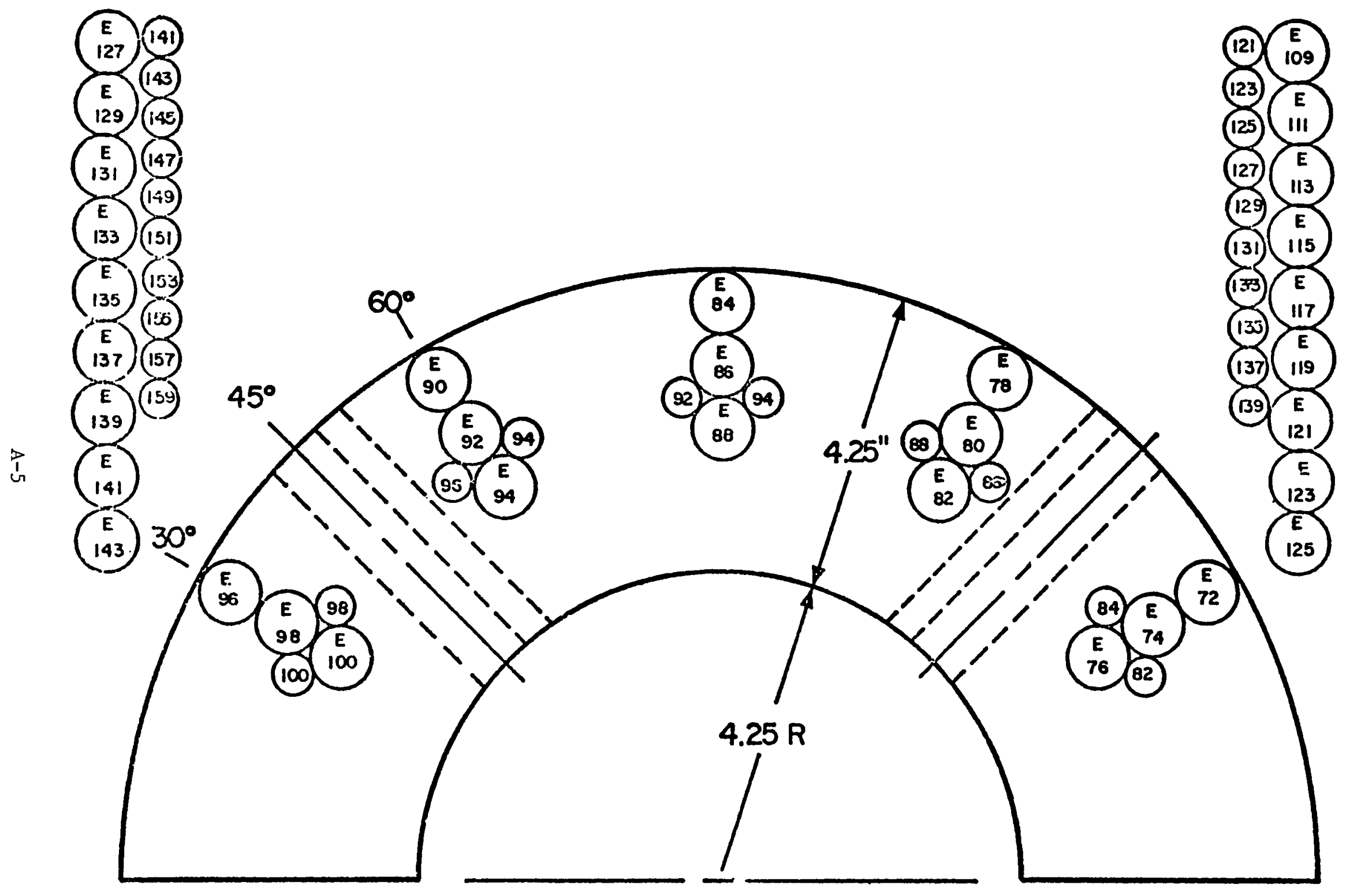

Fig. A-1. Edge Section 1BE (sheet 3 of 5) 
0

0 


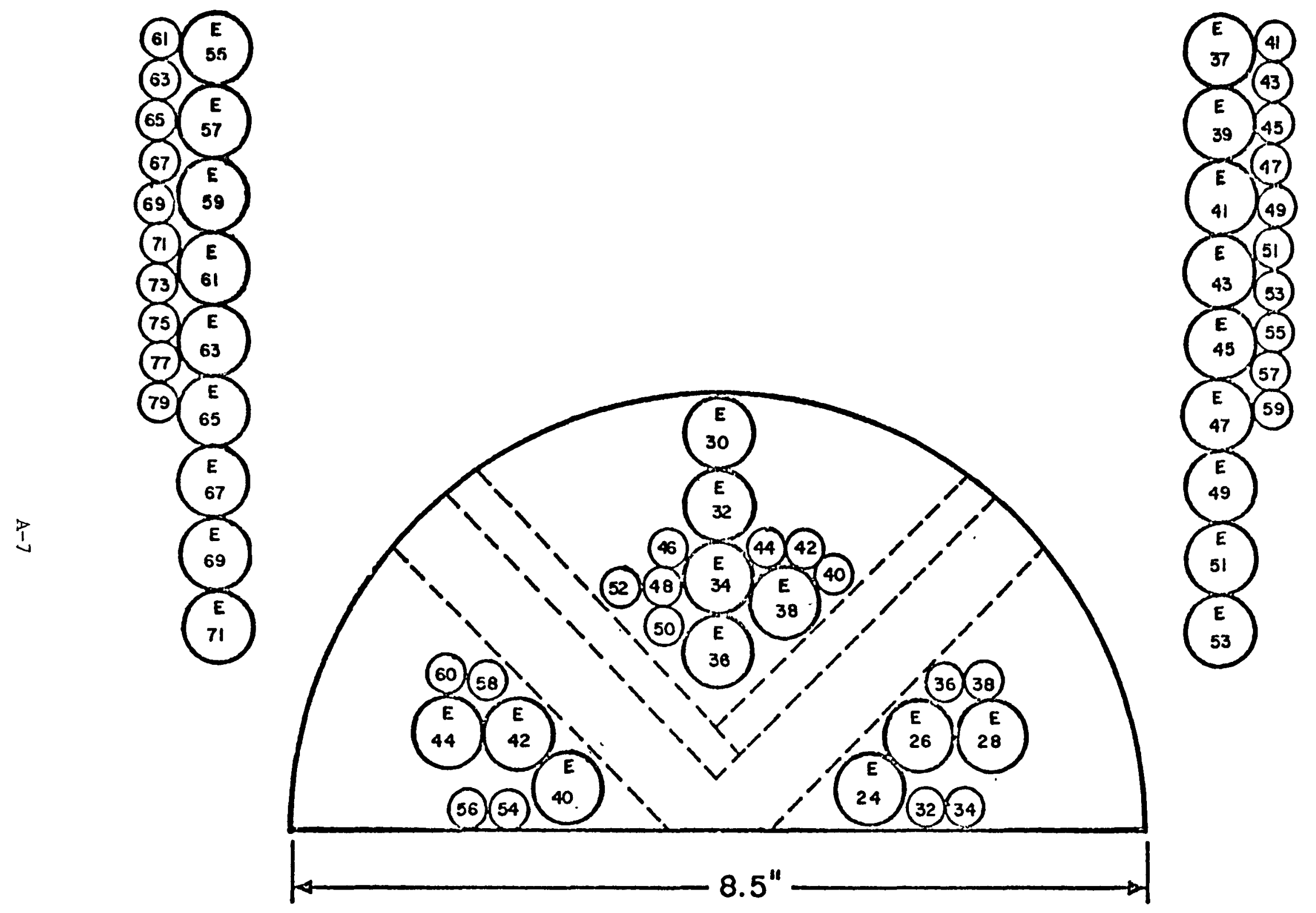

Fig. A-1. Center Section $1 \mathrm{BC}$ (sheet 5 of 5 ) 


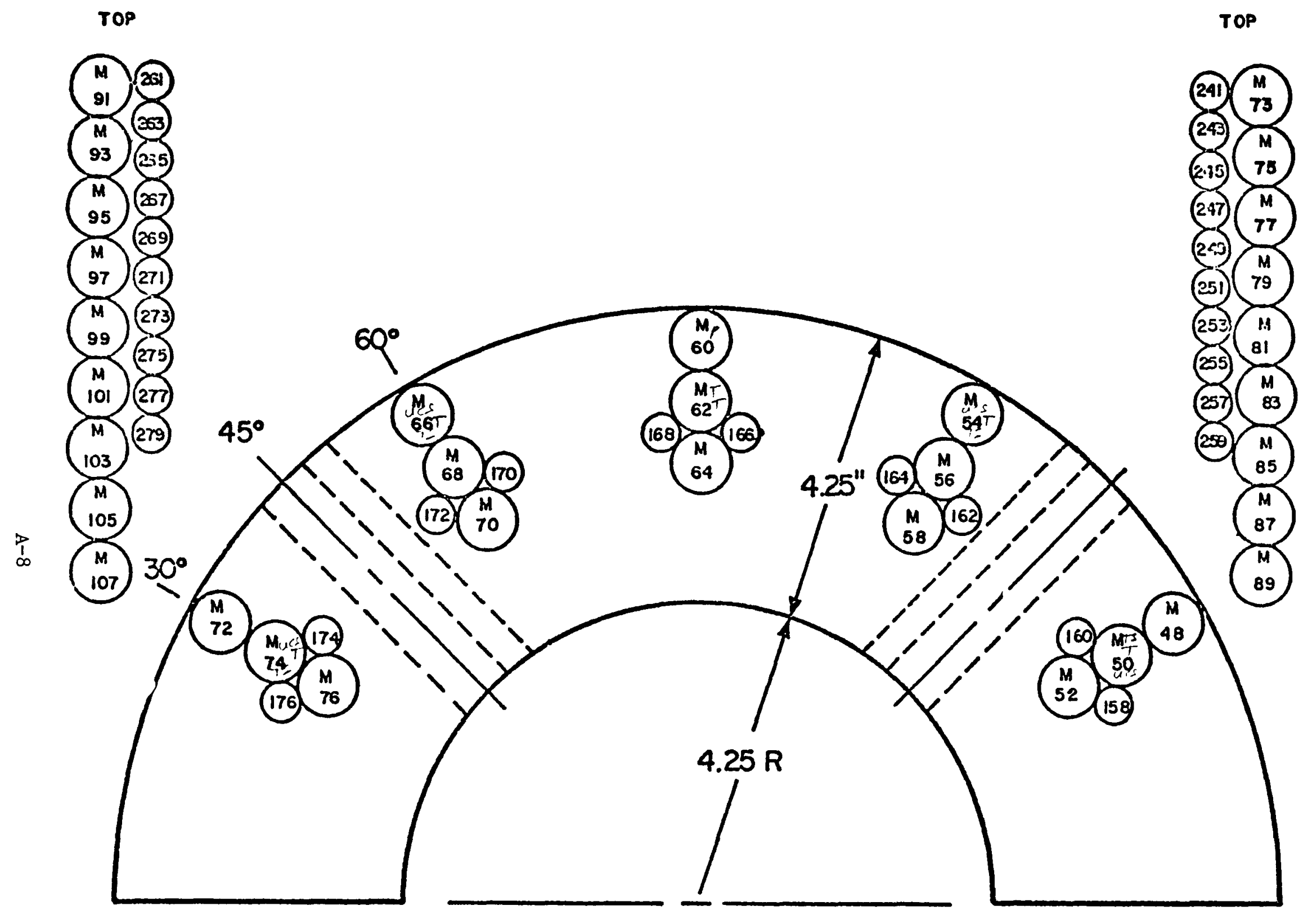

Fig. A-2. Edge Section $3 \mathrm{AE}$ (sheet 1 of 4) 


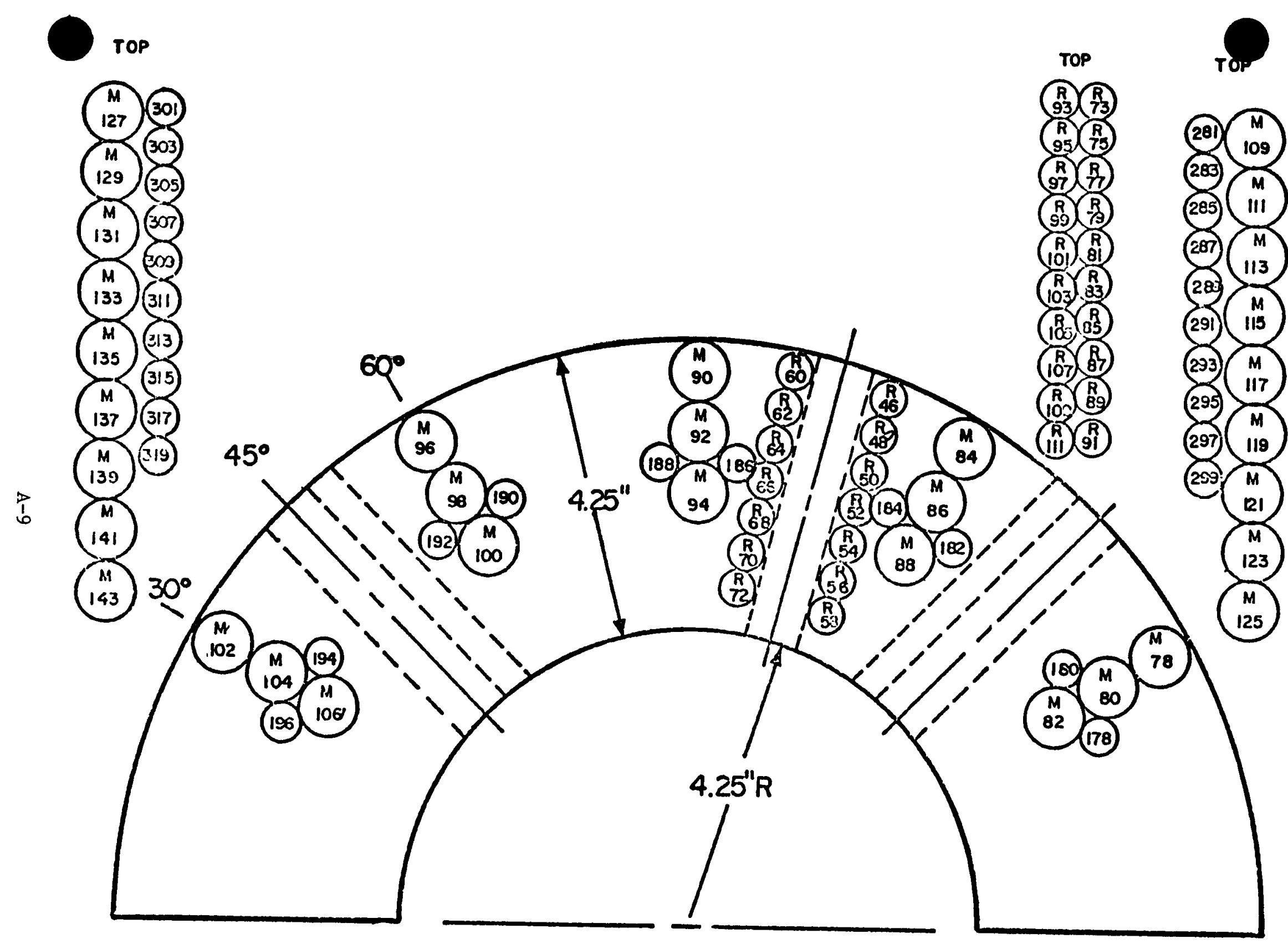

Fig. A-2. Edge Section 3BE (sheet 2 of 4) 


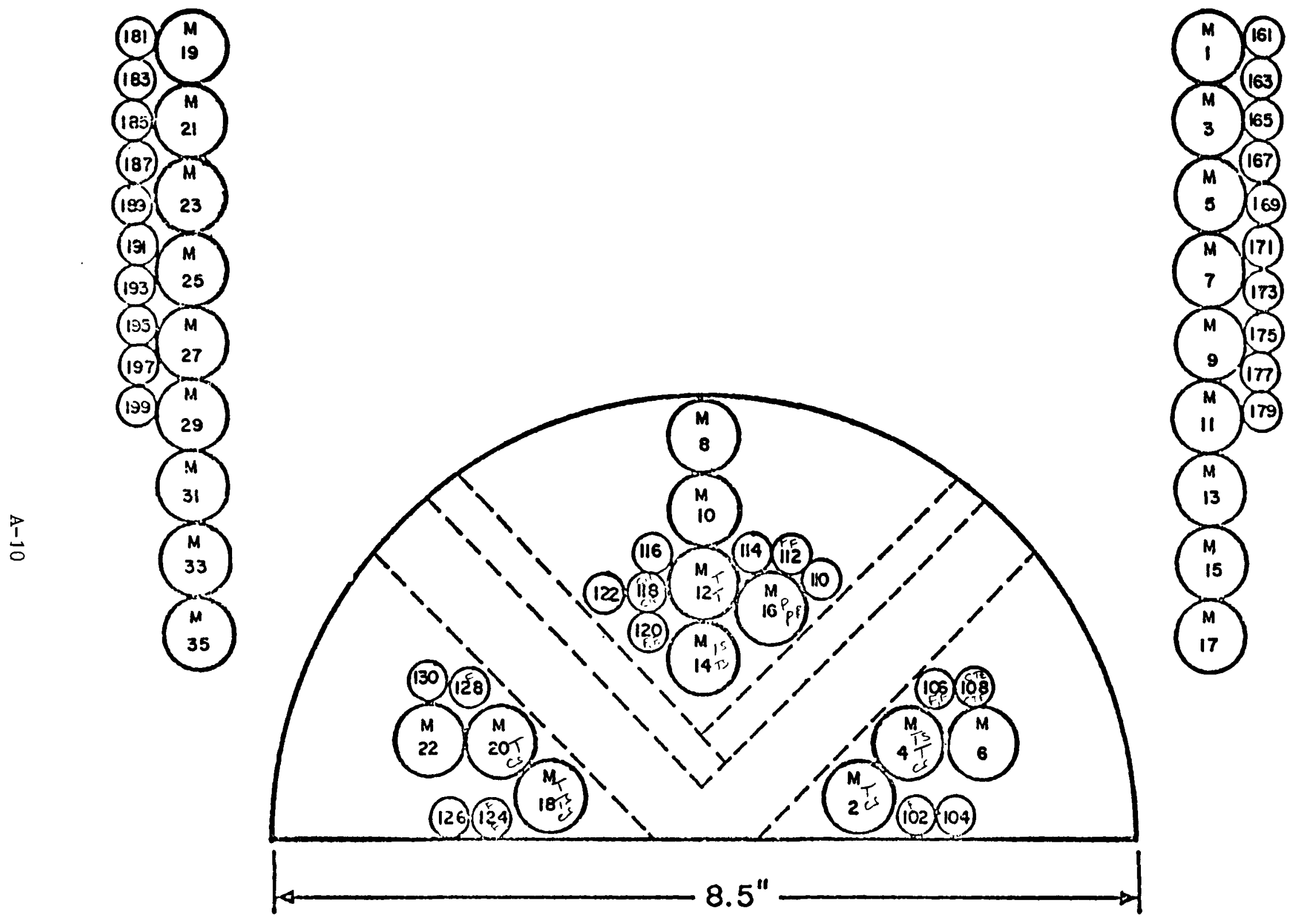

Fig. A-2. Center Section 3AC (sheet 3 of 4) 

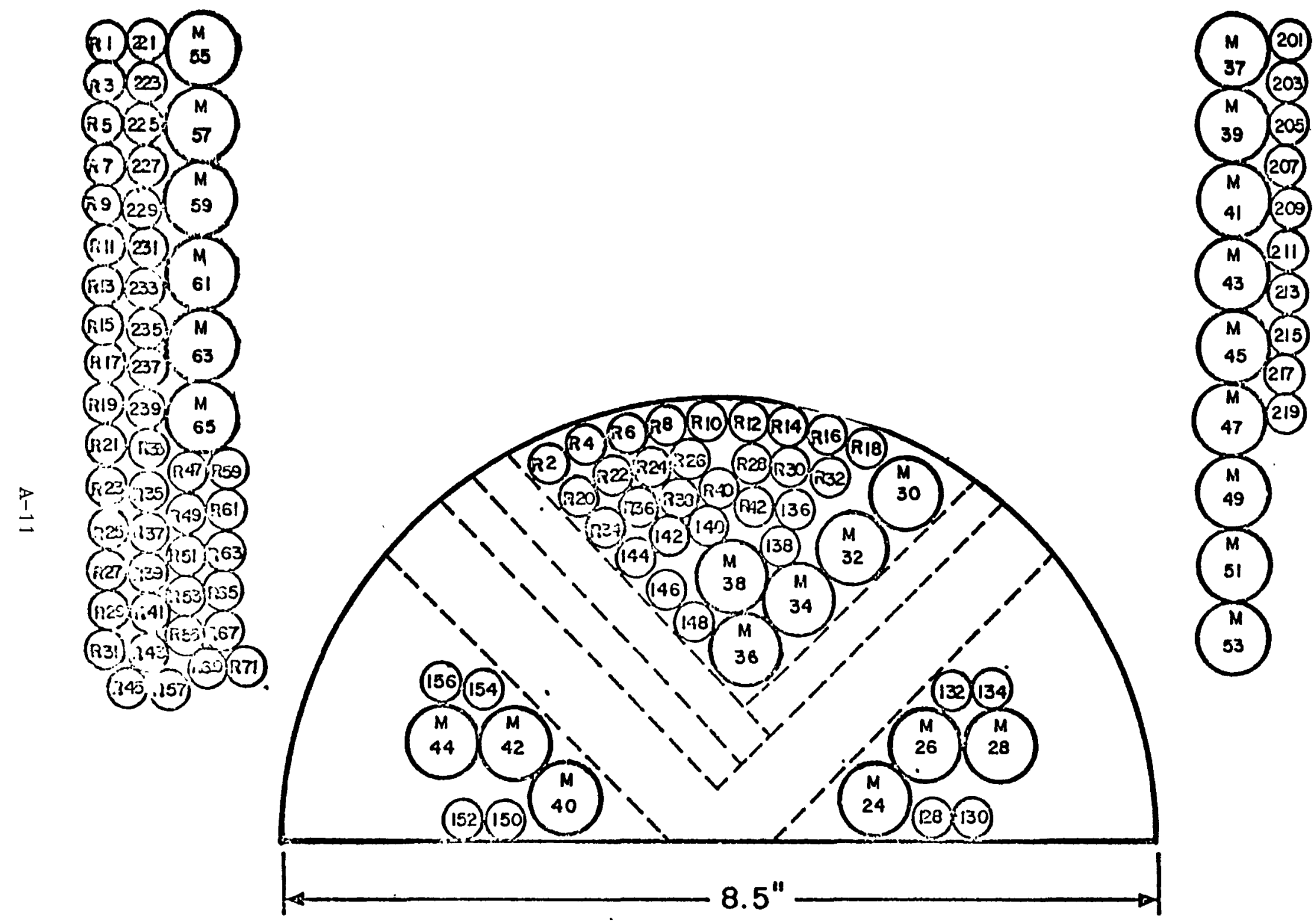

45

M 219

47219

$M$
49

$M$
51

Si

$M$
53

Fig. A-2. Center Section 3BC (sheet 4 of 4) 


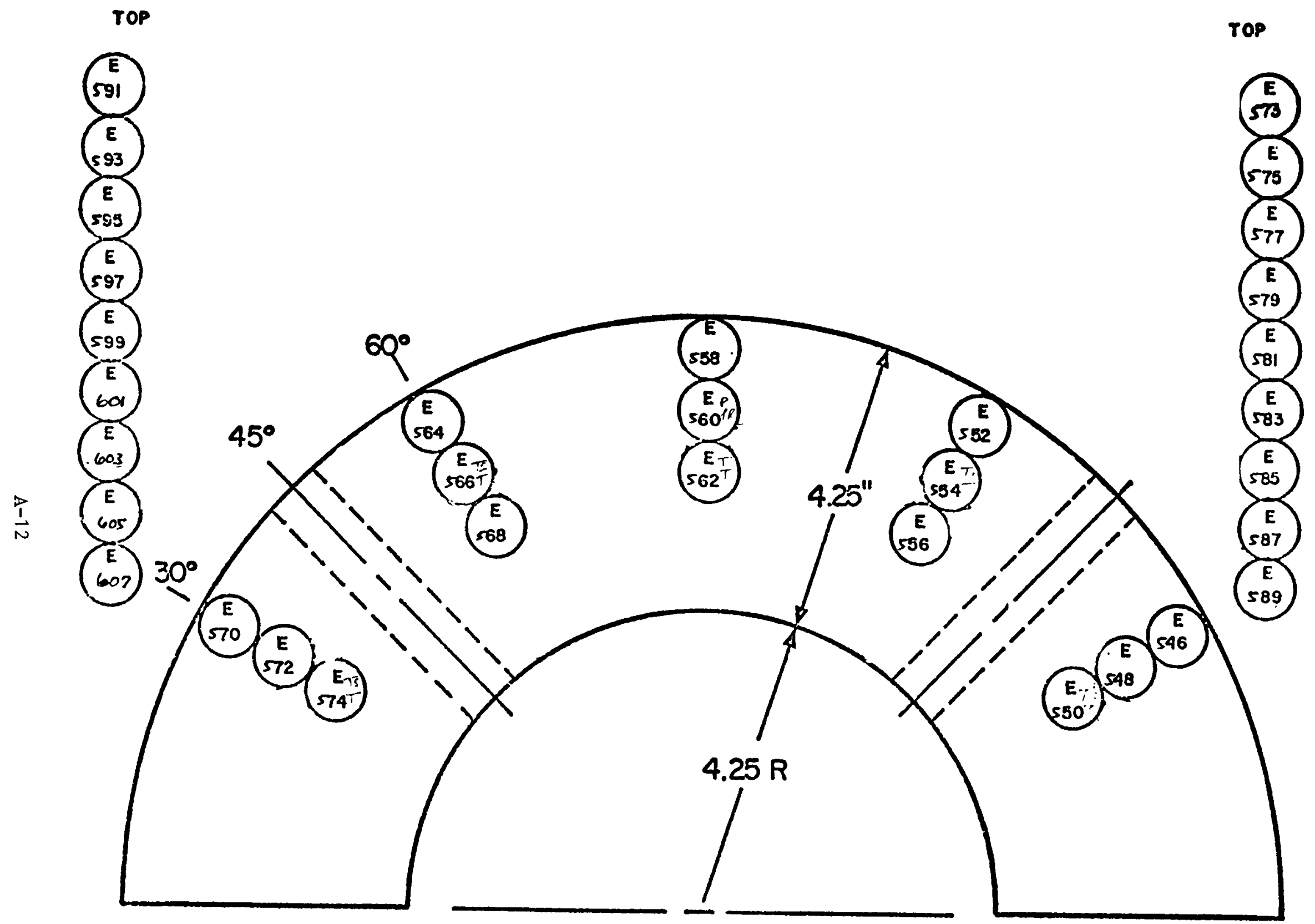

Fig. A-3. Edge Section 5AE (sheet 1 of 4) 


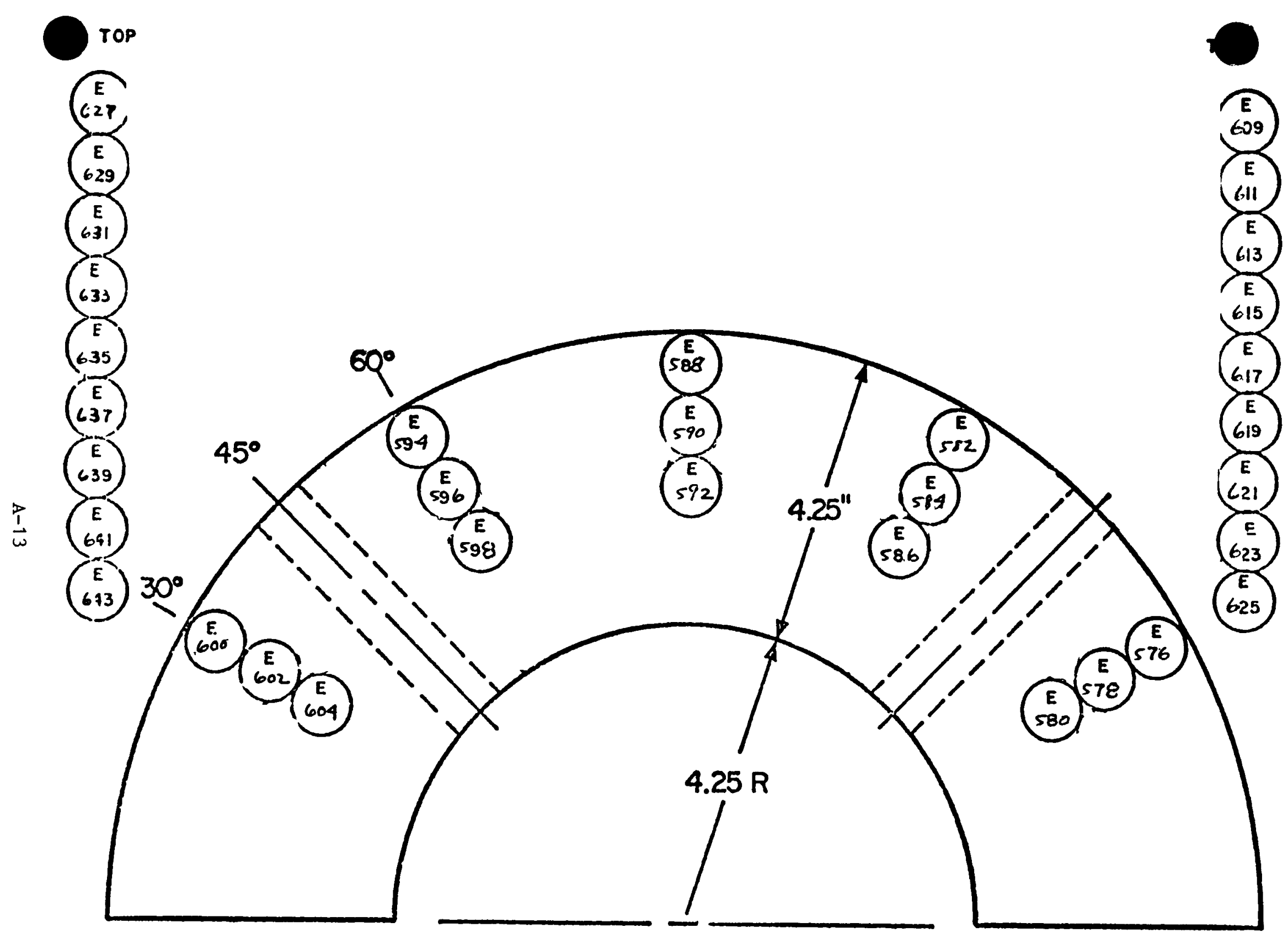

Fig. A-3. Edge Section 5BE (sheet 2 of 4) 


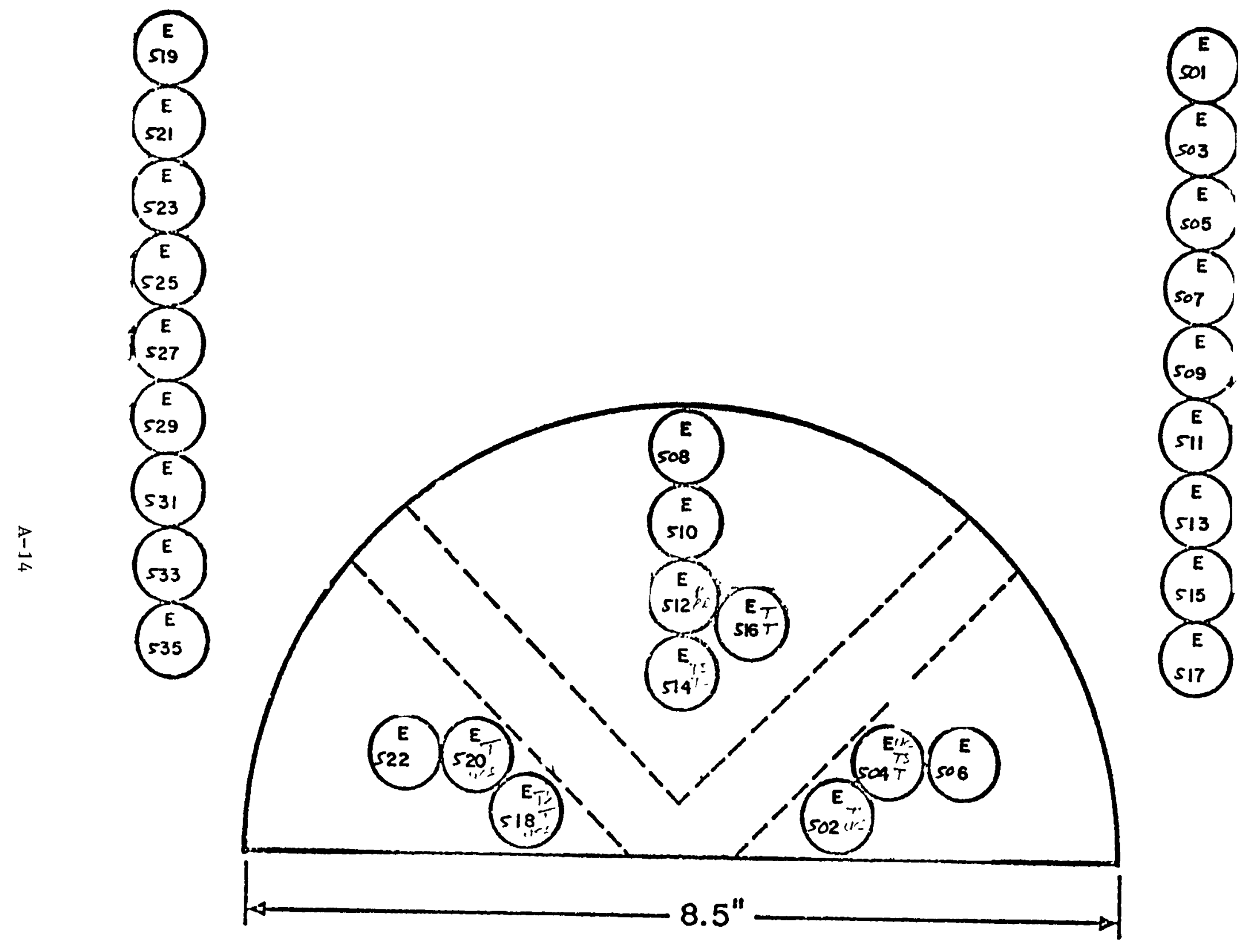

Fig. A-3. Center Section 5AC (sheet 3 of 4) 


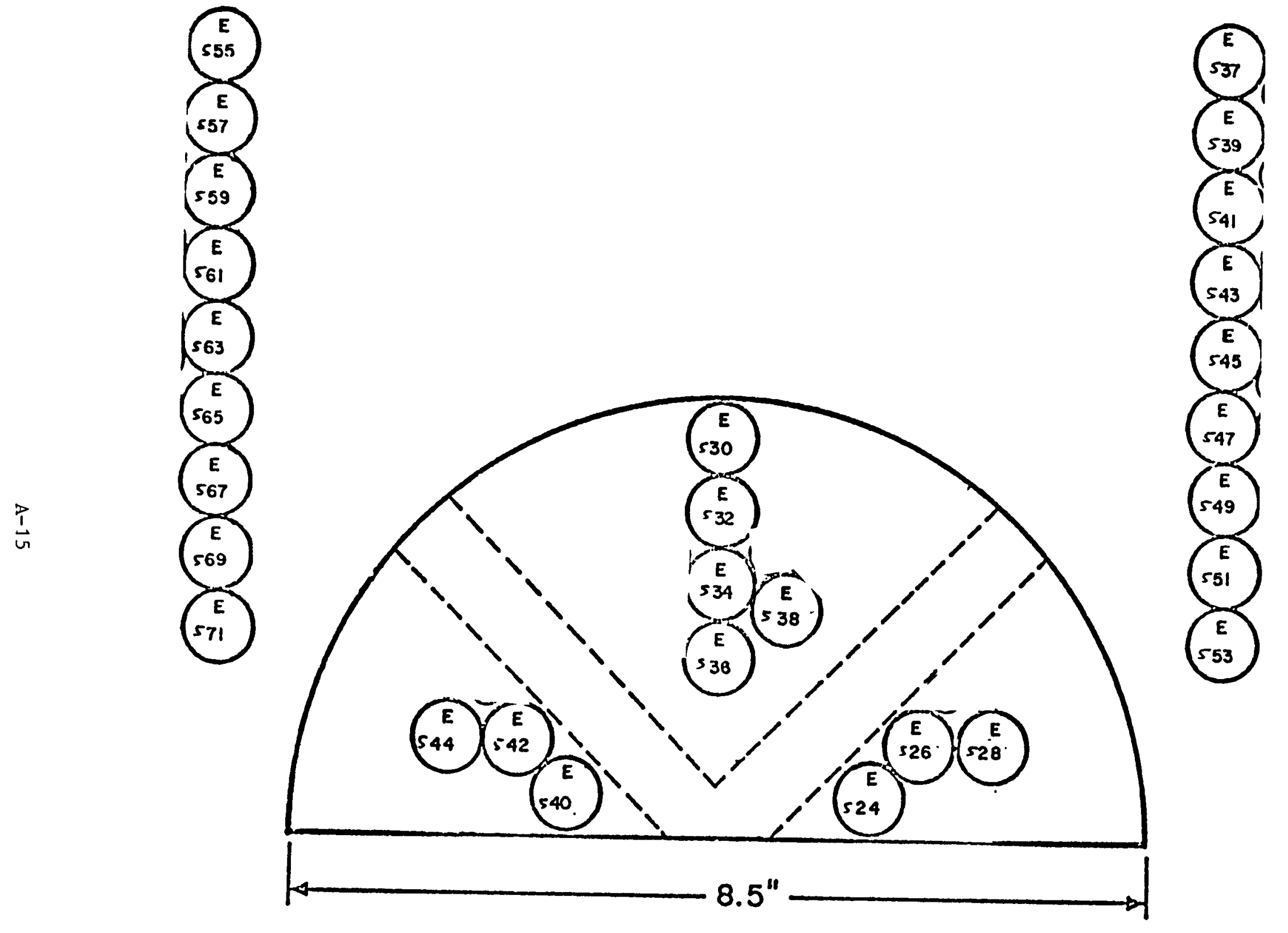

Fig. A-3. Center Section 5BC (sheet 4 of 4 ) 
APPENDIX B

INDIVIDUAL SPECIMEN DATA

B-1 
-

0 
TABLE B-1

TENSILE PROPERTIES OF H-451 GRAPHITE

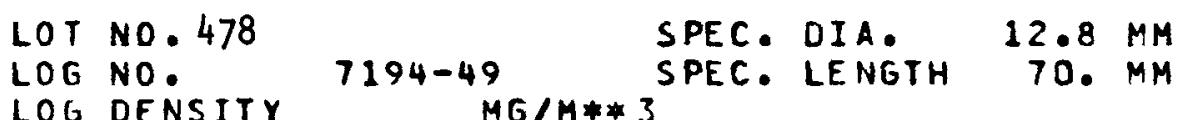

\begin{tabular}{|c|c|c|c|c|c|c|c|c|}
\hline $\begin{array}{l}\text { SPECIMEN } \\
\text { NUMBER }\end{array}$ & \multicolumn{2}{|c|}{$\begin{array}{l}\text { OR IENT- } \\
\text { A I ION }\end{array}$} & $\begin{array}{l}\text { LOCA- } \\
\text { TION }\end{array}$ & $\begin{array}{r}\text { DENSITY } \\
\text { (MG/M**3}\end{array}$ & $\begin{array}{l}\text { YOUNGS } \\
\text { IMODUL US } \\
\text { (GPA) }\end{array}$ & $\begin{array}{l}\text { PERM - } \\
\text { ANENT } \\
\text { SET } \\
\text { (PCT) }\end{array}$ & $\begin{array}{l}\text { FRAC- } \\
\text { TURE } \\
\text { STRAIN } \\
\text { (PCT) }\end{array}$ & $\begin{array}{l}\text { TENSILE } \\
\text { STRENGTH } \\
\text { (MPA) }\end{array}$ \\
\hline $\begin{array}{r}1 A C-E \\
- \\
- \\
- \\
- \\
- \\
- \\
- \\
- \\
- \\
1 B C- \\
- \\
- \\
- \\
- \\
- \\
- \\
- \\
- \\
-\end{array}$ & $\begin{array}{r}4 A \\
14 A \\
14 B \\
18 B \\
2 B \\
4 B \\
16 A \\
16 B \\
18 A \\
20 B \\
26 A \\
36 A \\
36 A \\
40 B \\
24 B \\
26 B \\
34 A \\
34 B \\
40 A \\
42 B\end{array}$ & $\begin{array}{l}A X \\
A X \\
A X \\
A X \\
A X \\
A X \\
A X \\
A X \\
A X \\
A X \\
A X \\
A X \\
A X \\
A X \\
A X \\
A X \\
A X \\
A X \\
A X \\
A X\end{array}$ & $\begin{array}{l}E C \\
E C \\
E C \\
E C \\
E C \\
E C \\
E C \\
E C \\
E C \\
E C \\
E C \\
E C \\
E C \\
E C \\
E C \\
E C \\
E C \\
E C \\
E C \\
E C\end{array}$ & $\begin{array}{l}1.736 \\
1.738 \\
1.731 \\
1.732\end{array}$ & $\begin{array}{l}8.4 \\
8.9 \\
9.2 \\
8.6\end{array}$ & $\begin{array}{l}.009 \\
.030 \\
.012 \\
.017\end{array}$ & $\begin{array}{l}.192 \\
.231 \\
.227 \\
.221\end{array}$ & $\begin{array}{l}12.2 \\
13.7 \\
14.7 \\
13.5 \\
14.0 \\
14.3 \\
15.8 \\
14.4 \\
14.7 \\
11.5 \\
12.7 \\
10.7 \\
14.6 \\
11.7 \\
15.7 \\
13.9 \\
12.7 \\
12.7 \\
10.6 \\
14.5\end{array}$ \\
\hline & & MEA & & 1.735 & $\begin{array}{r}8.5 \\
11.24 \mathrm{M}\end{array}$ & .017 & .209 & $\begin{array}{l}13.4 \\
1946 . P S I)\end{array}$ \\
\hline & & STO & DEV. & .003 & $1.09 \mathrm{M}$ & (PSI) & .024 & $\begin{array}{l}1.5 \\
222 . P S I)\end{array}$ \\
\hline
\end{tabular}


TABLE B-1 (Continued)

TENSILE PROPERTIES OF H 451 GRAPHITE
LOT NO. 478
LOG NO.
LOG DENSITY

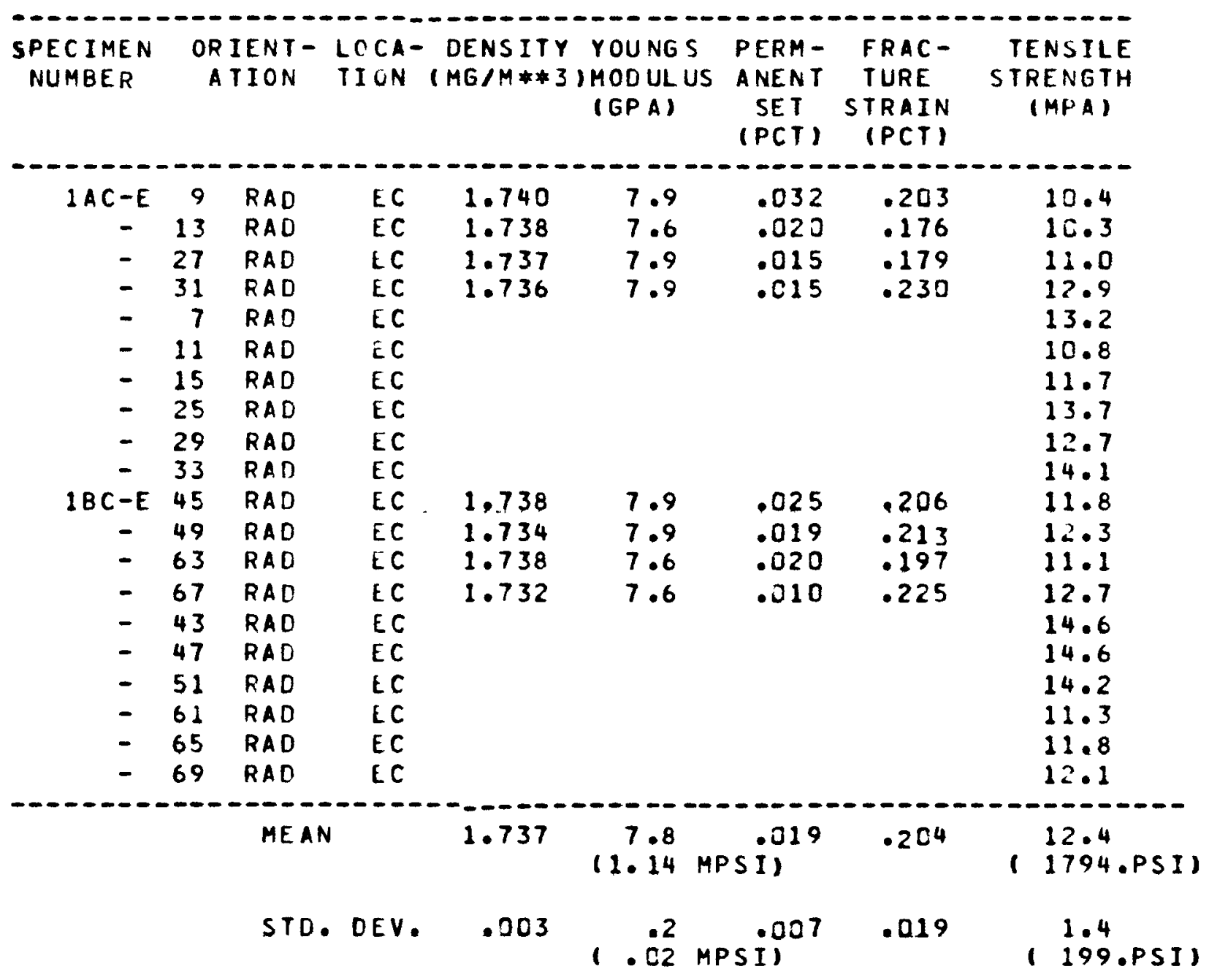


TABLE B-1 (Continued)

TENSILE PROPERTIES OF H-451 GRAPHITE

$\begin{array}{llll}\text { LOT NO. } 478 & & \text { SPEC. DIA. } & 12.8 \mathrm{MM} \\ \text { LOG NO. } & 7194-49 & \text { SPEC. LENGTH } & 70 . \mathrm{MM}\end{array}$ LOG DENSIIY ....... MG/M**3

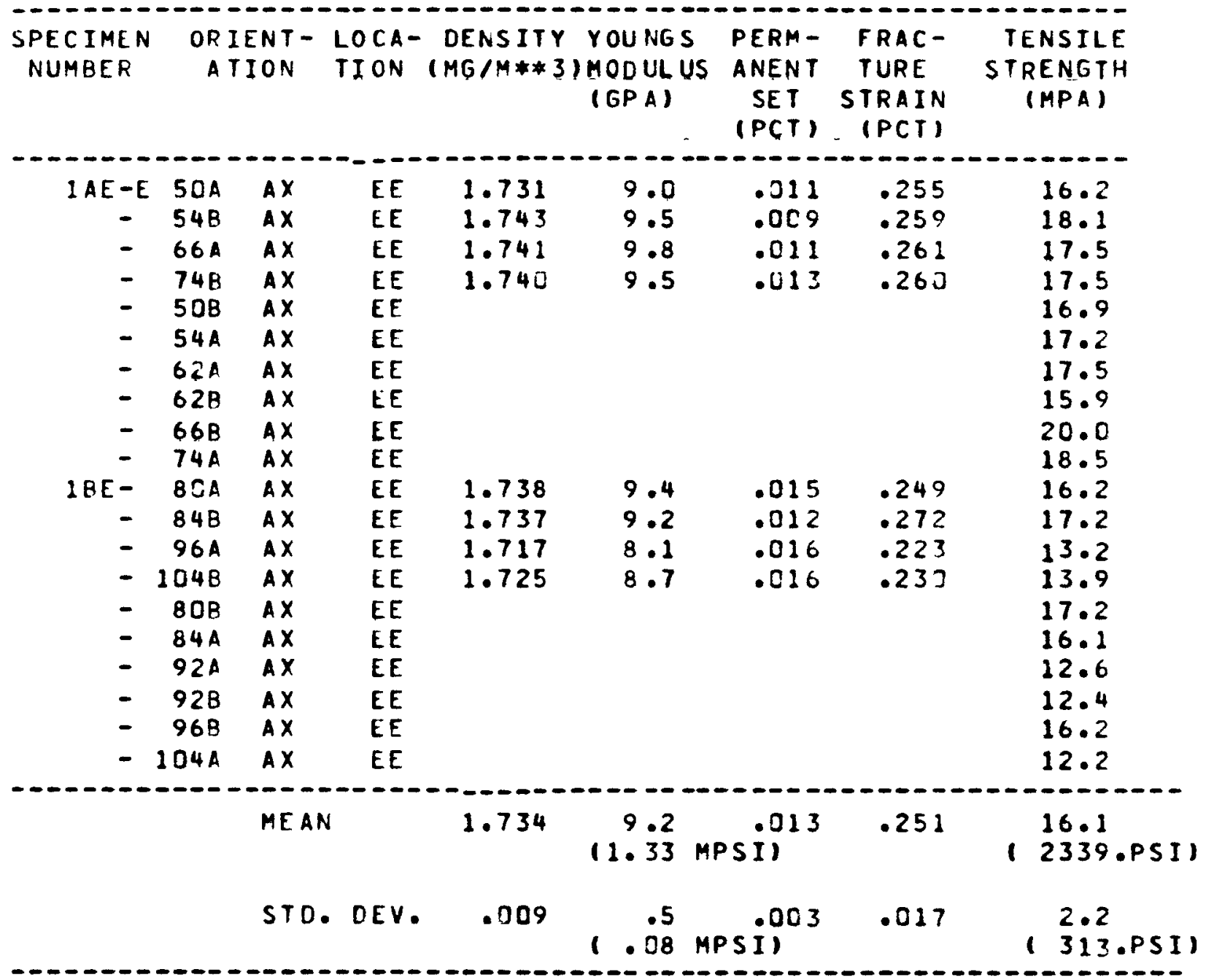




\section{TABLE B-1 (Continued)}

TENSILE PROPERTIES OF H-451 GRAPHITE

LOT NO. 478

LOG NO.

LOG DENSITY
SPEC. DIA.

SPEC. LENGTH 70.8 MM

\begin{tabular}{|c|c|c|c|c|c|c|c|c|}
\hline $\begin{array}{l}\text { SPECIMEN } \\
\text { NUMBER }\end{array}$ & \multicolumn{2}{|c|}{$\begin{array}{l}\text { ORIENT- } \\
\text { ATION }\end{array}$} & $\begin{array}{l}\text { LOCA- } \\
\text { TION }\end{array}$ & $\begin{array}{l}\text { DENS ITY } \\
\text { I MG/M**3 }\end{array}$ & $\begin{array}{l}\text { YOUNG } \\
\text { IMODUL US } \\
\text { (GPA) }\end{array}$ & $\begin{array}{l}\text { PERM - } \\
\text { ANENT } \\
\text { SET } \\
\text { (PCT) }\end{array}$ & $\begin{array}{l}\text { FRAC- } \\
\text { TURE } \\
\text { STRAIN } \\
\text { (PCT) }\end{array}$ & $\begin{array}{l}\text { TENSILE } \\
\text { STRENGTH } \\
\text { (MPA) }\end{array}$ \\
\hline $\begin{array}{r}1 A E-E \\
- \\
- \\
- \\
= \\
= \\
- \\
= \\
1 B E- \\
- \\
- \\
- \\
- \\
- \\
- \\
- \\
-\end{array}$ & $\begin{array}{l}79 \\
83 \\
99 \\
103 \\
77 \\
81 \\
85 \\
97 \\
101 \\
105 \\
115 \\
119 \\
133 \\
137 \\
113 \\
117 \\
121 \\
131 \\
135 \\
139\end{array}$ & $\begin{array}{l}\text { RAD } \\
\text { RAD } \\
\text { RAD } \\
\text { RAD } \\
\text { RAD } \\
\text { RAD } \\
\text { RAD } \\
\text { RAD } \\
\text { RAD } \\
\text { RAD } \\
\text { RAD } \\
\text { RAD } \\
\text { RAD } \\
\text { RAD } \\
\text { RAD } \\
\text { RAD } \\
\text { RAD } \\
\text { RAD } \\
\text { RAD } \\
\text { RAD }\end{array}$ & $\begin{array}{l}E E \\
L E \\
E E \\
\text { EE } \\
E E \\
\text { EE } \\
E E \\
E E \\
E E \\
E E \\
E E \\
\text { EE } \\
\text { LE } \\
E E \\
E E \\
\text { LE } \\
E E \\
\text { EE } \\
L E \\
L E\end{array}$ & $\begin{array}{l}1.736 \\
1.739 \\
1.738 \\
1.742\end{array}$ & $\begin{array}{l}7.8 \\
8.2 \\
7.7 \\
8.3\end{array}$ & $\begin{array}{l}.021 \\
.0220 \\
.016 \\
.015\end{array}$ & $\begin{array}{l}.296 \\
.287 \\
.278 \\
.286\end{array}$ & $\begin{array}{l}15.9 \\
15.4 \\
15.2 \\
15.6 \\
15.3 \\
16.6 \\
20.1 \\
15.3 \\
15.5 \\
15.4 \\
16.2 \\
16.5 \\
13.8 \\
13.3 \\
16.6 \\
14.1 \\
14.1 \\
15.5 \\
16.0 \\
13.8\end{array}$ \\
\hline & & ME A & $v$ & 1.735 & $\begin{array}{r}8.0 \\
11.16\end{array}$ & PSII & .279 & $(2249 . P S I)$ \\
\hline & & STI & DEV & .006 & 1.04 & PSII & .024 & ( $\begin{array}{l}1.5 \\
211 . P S I)\end{array}$ \\
\hline
\end{tabular}


TABLE B-1 (Continued)

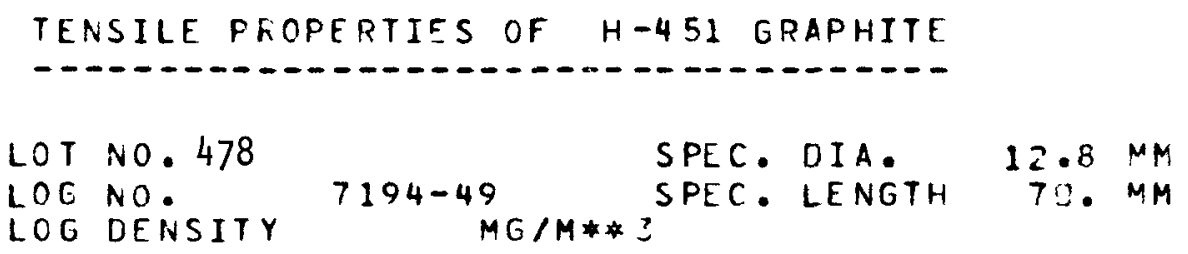

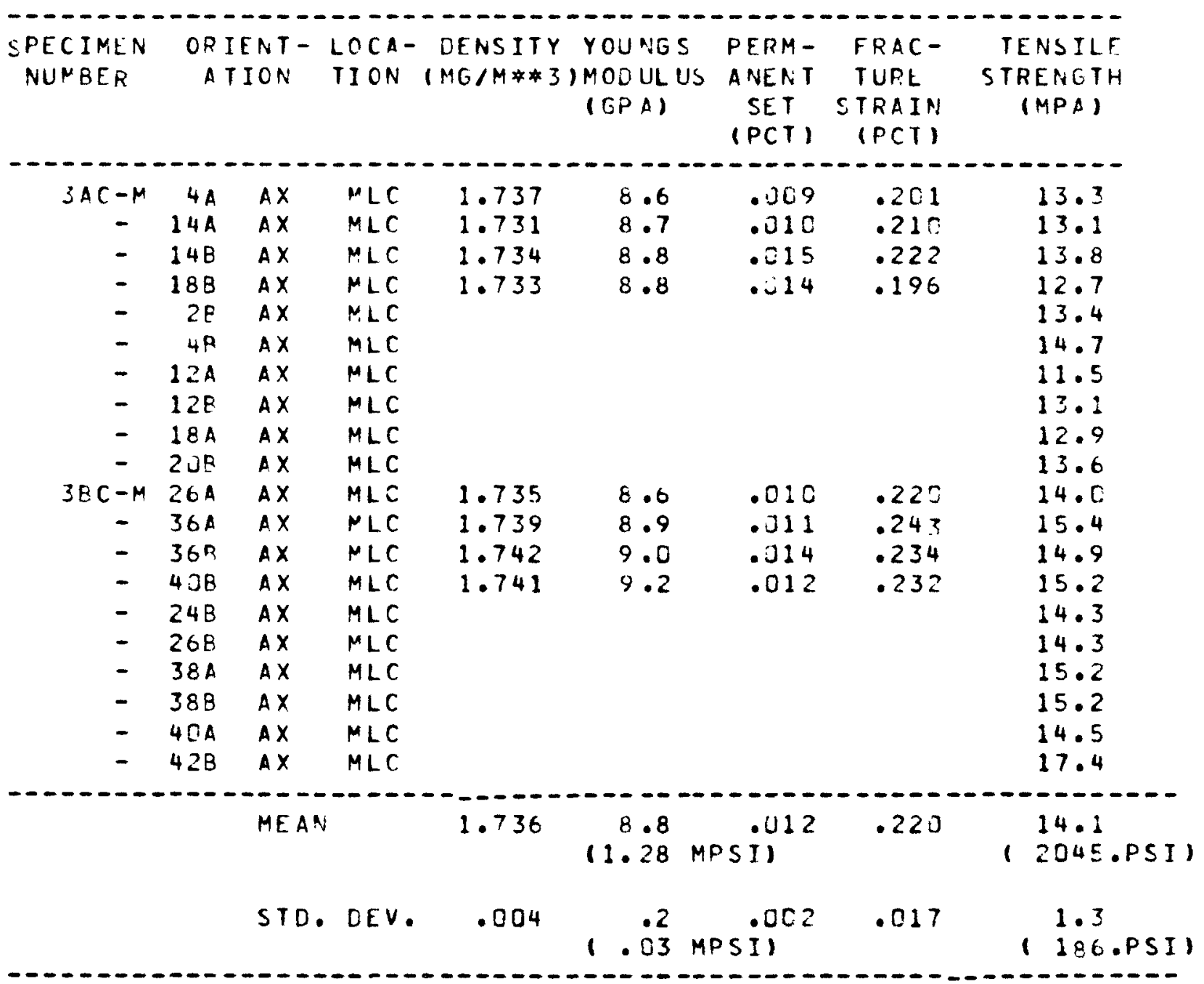


TABLE B-1 (Continued)

TENSILE PROPERTIES OF H-451 GRAPHITE

TENSILE PROPERTIES OF

LOT NO. 478 SPEC. DIA. $12.8 \mathrm{MM}$

LOG NO. 7194-49 SPEC. LENGTH 70. MM

LOG DENSITY MG/M* 3

\begin{tabular}{|c|c|c|c|c|c|c|c|c|}
\hline $\begin{array}{l}\text { SPECIMEN } \\
\text { NUMBER }\end{array}$ & & $\begin{array}{l}\text { IENT- } \\
\text { TION }\end{array}$ & $\begin{array}{l}\text { LOCA- } \\
\text { TION }\end{array}$ & $\begin{array}{l}\text { DENSITY } \\
\text { (MG/M**3 }\end{array}$ & $\begin{array}{l}\text { YOUNGS } \\
\text { MODUL US } \\
\text { (GPA) }\end{array}$ & $\begin{array}{l}\text { PERM - } \\
\text { ANENT } \\
\text { SET } \\
\text { (PCT) }\end{array}$ & $\begin{array}{l}\text { FRAC - } \\
\text { TURE } \\
\text { STRAIN } \\
\text { (PCT) }\end{array}$ & $\begin{array}{l}\text { TENSILE } \\
\text { STRENGTH } \\
\text { (MPA) }\end{array}$ \\
\hline$\ldots-n$ & $-\infty$ & $-\pi$ & $=$ & $\ldots$ & ---0 & $\cdots-\cdots$ & $--\infty-\infty$ & 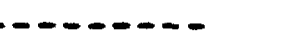 \\
\hline $3 A C-M$ & 9 & RAD & $M L C$ & 1.738 & 7.9 & . & .230 & 13.1 \\
\hline- & 27 & RAD & $M L C$ & 1.728 & 7.7 & .020 & .182 & $1 \mathrm{C} .3$ \\
\hline - & & RAD & $M L C$ & 1.730 & $7 \cdot 3$ & .021 & .124 & $7 \cdot 2$ \\
\hline - & 7 & RAD & MLC & & & & & 13.2 \\
\hline & & RAD & $M L C$ & & & & & 13.0 \\
\hline & & RAD & $M L C$ & & & & & 10.7 \\
\hline & & RAD & MLC & & & & & $12 \cdot 1$ \\
\hline & 29 & RAD & MLC & & & & & 10.6 \\
\hline - & 33 & RAD & $M L C$ & & & & & $12 \cdot 0$ \\
\hline $3 B C-M$ & 45 & RAD & MLC & 1.739 & 7.7 & .021 & .163 & 10.2 \\
\hline - & 49 & RAD & MLC & 1.739 & 7.6 & .014 & .186 & 10.9 \\
\hline - & 57 & RAD & $M L C$ & 1.737 & 7.7 & .012 & .240 & 13.1 \\
\hline & & RAD & MLC & 1.740 & 7.6 & .017 & .231 & 12.7 \\
\hline - & 43 & RAD & $M L C$ & & & & & 12.7 \\
\hline & 47 & RAD & $M L C$ & & & & & 12.6 \\
\hline - & 51 & RAD & $M L C$ & & & & & 10.9 \\
\hline & 55 & RAD & MLC & & & & & 13.6 \\
\hline - & 59 & RAD & $M L C$ & & & & & 13.0 \\
\hline - & 63 & RAD & $M L C$ & & & & & 10.3 \\
\hline & & . & --1 & & & $\ldots$ & $\cdots$ & $\cdots \cdots \cdots-\cdots$ \\
\hline & & MEAI & & 1.736 & 7.7 & .017 & 199 & $\begin{array}{l}11.7 \\
1698 . P S T,\end{array}$ \\
\hline & & STO. & DEV. & .004 & $1.03 N$ & Sil) & .038 & $\left(\begin{array}{l}1.6 \\
227 . P S I)\end{array}\right.$ \\
\hline
\end{tabular}




\section{TABLE B-1 (Continued)}

TENSILE PROPERTIES OF H-451 GRAPHITE

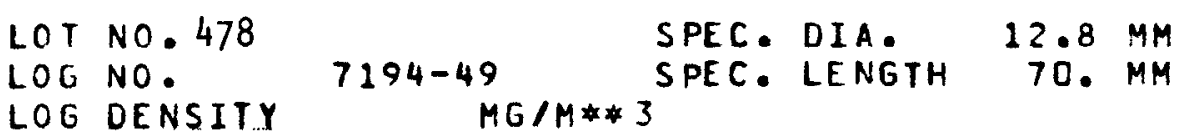

\begin{tabular}{|c|c|c|c|c|c|c|c|c|}
\hline $\begin{array}{l}\text { SPECIMEN } \\
\text { NUMBER }\end{array}$ & \multicolumn{2}{|c|}{$\begin{array}{l}\text { OR IENT - } \\
\text { A TION }\end{array}$} & $\begin{array}{l}\text { LOCA- } \\
\text { TION }\end{array}$ & $\begin{array}{l}\text { DENSITY } \\
(M G / M * * 3)\end{array}$ & $\begin{array}{l}\text { YOUNG S } \\
\text { IMOUUL US } \\
\text { (GPA) }\end{array}$ & $\begin{array}{l}\text { PERM - } \\
\text { ANEN T } \\
\text { SET } \\
\text { (PCT) }\end{array}$ & $\begin{array}{l}\text { FRAC- } \\
\text { TURE } \\
\text { STRAIN } \\
\text { (PCT) }\end{array}$ & $\begin{array}{l}\text { TENSILE } \\
\text { STRENGTH } \\
\text { (MPA) }\end{array}$ \\
\hline-- & -- & $=$ & & & ---- & $\cdots$ & --- & $-\cdots-$ \\
\hline $\begin{array}{c}3 A E-M \\
-\end{array}$ & $\begin{array}{l}5 C A \\
54 R\end{array}$ & $\begin{array}{l}A X \\
A X\end{array}$ & $\begin{array}{l}\text { MLE } \\
\text { MLE }\end{array}$ & $\begin{array}{l}1.745 \\
1.743\end{array}$ & $\begin{array}{l}10.0 \\
10.0\end{array}$ & $\begin{array}{l}.013 \\
.016\end{array}$ & $\begin{array}{l}.29 ? \\
.275\end{array}$ & $\begin{array}{l}19 \cdot 1 \\
18.5\end{array}$ \\
\hline - & 66A & $A X$ & MLE & 1.739 & 9.6 & .024 & .273 & 17.8 \\
\hline - & $\begin{array}{l}74 B \\
50 B\end{array}$ & $\begin{array}{l}A X \\
A X\end{array}$ & $\begin{array}{l}\text { MLE } \\
\text { MLE }\end{array}$ & 1.733 & 9.4 & .010 & .274 & $\begin{array}{l}18.0 \\
19.6\end{array}$ \\
\hline - & $54 \mathrm{~A}$ & $A X$ & MLE & & & & & 17.6 \\
\hline - & $62 \mathrm{~A}$ & $A X$ & MLE & & & & & 15.9 \\
\hline - & 628 & $A X$ & MLE & & & & & $17 \cdot 3$ \\
\hline - & $\begin{array}{l}66 B \\
74 A\end{array}$ & $\begin{array}{l}A X \\
A X\end{array}$ & $\begin{array}{l}\text { MLE } \\
M L E\end{array}$ & & & & & $\begin{array}{l}16.1 \\
18.8\end{array}$ \\
\hline 3BE- & $80 A$ & $A X$ & MLE & 1.742 & $10 \cdot 0$ & .015 & .259 & 17.6 \\
\hline - & $86 \mathrm{~B}$ & $A X$ & MLE & 1.749 & 10.0 & .011 & .289 & 19.4 \\
\hline - & $96 \mathrm{~A}$ & $A X$ & MLE & 1.754 & 10.0 & .009 & .296 & 20.2 \\
\hline - & $104 \mathrm{~B}$ & $A X$ & MLE & 1.741 & 9.1 & .010 & .274 & 18.7 \\
\hline - & $8 O B$ & $A X$ & $M L E$ & & & & & 19.9 \\
\hline - & $84 \mathrm{~A}$ & $A X$ & MLE & & & & & 18.7 \\
\hline & $92 A$ & $A X$ & MLE & & & & & 20.4 \\
\hline & $92 \mathrm{~B}$ & $A X$ & $M L E$ & & & & & 18.8 \\
\hline & $96 \mathrm{~B}$ & $A X$ & MLE & & & & & 20.6 \\
\hline - & $104 \mathrm{~A}$ & $A X$ & MLE & & & & & 19.0 \\
\hline- & $-\infty$ & 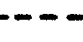 & $-\infty$ & & & -7 & - & $-\cdots-\cdots$ \\
\hline & & MEA & & 1.743 & $\begin{array}{r}9.8 \\
11.41 \mathrm{M}\end{array}$ & PSIJ & .279 & $\begin{array}{l}18.6 \\
(2698 . P S I)\end{array}$ \\
\hline & & STD & DEV. & .006 & 1.04 & (PSI) & .012 & $\left(\begin{array}{l}1.3 \\
187 . P S I)\end{array}\right.$ \\
\hline
\end{tabular}


TABLE B-1 (Continued)

TENSILE PROPERTIES OF H-451 GRAPHITE

TENSILE PROPERTIES OF

\begin{tabular}{|c|c|c|c|c|c|}
\hline LOT & $\begin{array}{l}\text { No. } 478 \\
\text { No. }\end{array}$ & 7194 & $\begin{array}{l}\text { SPEC. } \\
\text { SPEC. }\end{array}$ & $\begin{array}{l}\text { DIA. } \\
\text { LENGTH }\end{array}$ & $\begin{array}{r}12.8 \mathrm{MM} \\
70 . \mathrm{MM}\end{array}$ \\
\hline ? & NSITY & & & & \\
\hline
\end{tabular}

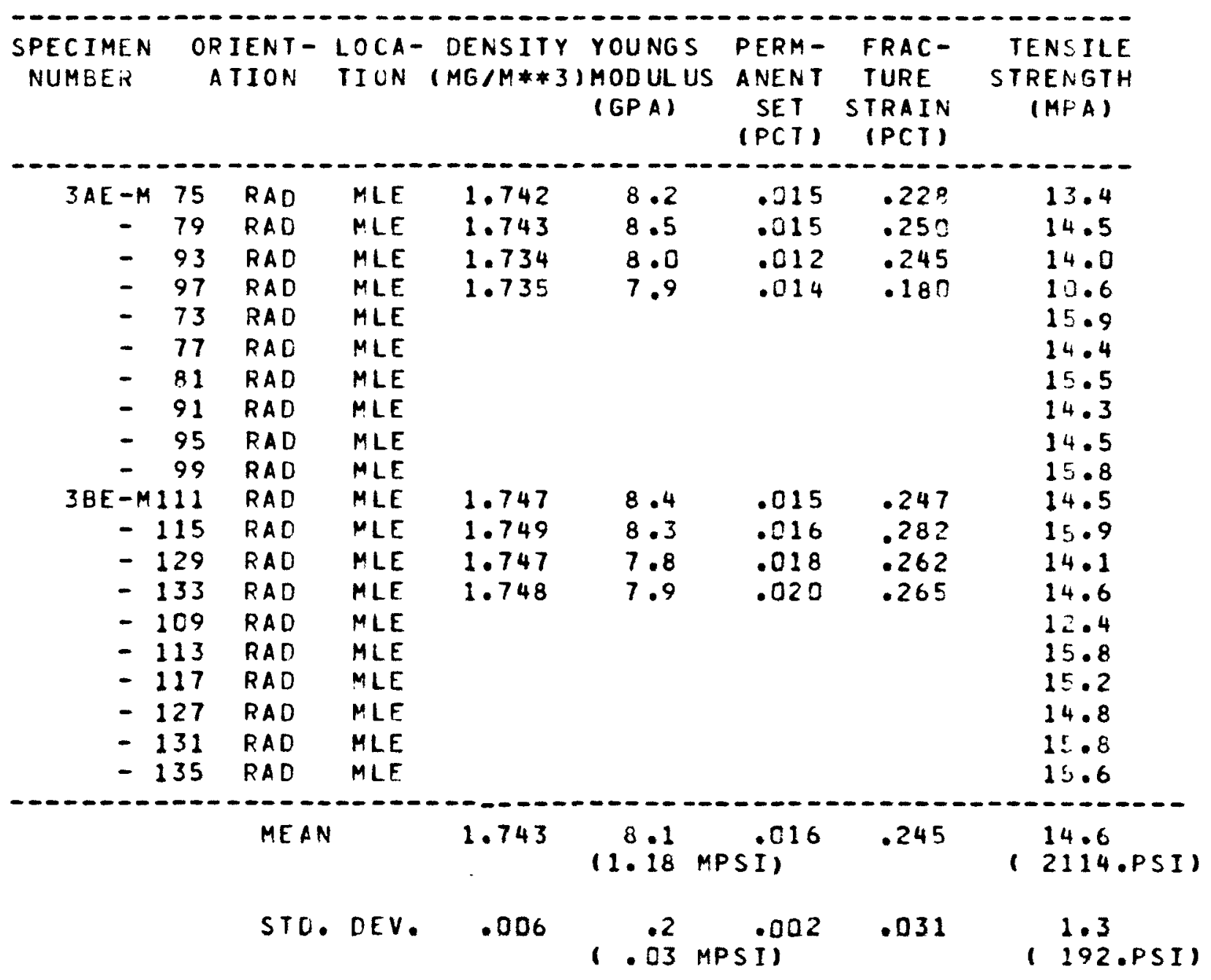


TABLE B-2

TENSILE PROPERTIES OF H-451 GRAPHITE

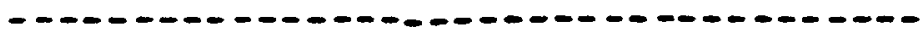

$\begin{array}{lllr}\text { LOT NO.478 } & & \text { SPEC. DIA. } & 12.8 \mathrm{MH} \\ \text { LOG NO. } & 7194-52 & \text { SPEC. LENGTH } & 70 . \mathrm{MM}\end{array}$

$\begin{array}{lllr}\text { LOT NO.478 } & & \text { SPEC. DIA. } & 12.8 \mathrm{MH} \\ \text { LOG NO. } & 7194-52 & \text { SPEC. LENGTH } & 70 . \mathrm{MM}\end{array}$

LOG DENSITY $M G / M * * 3$

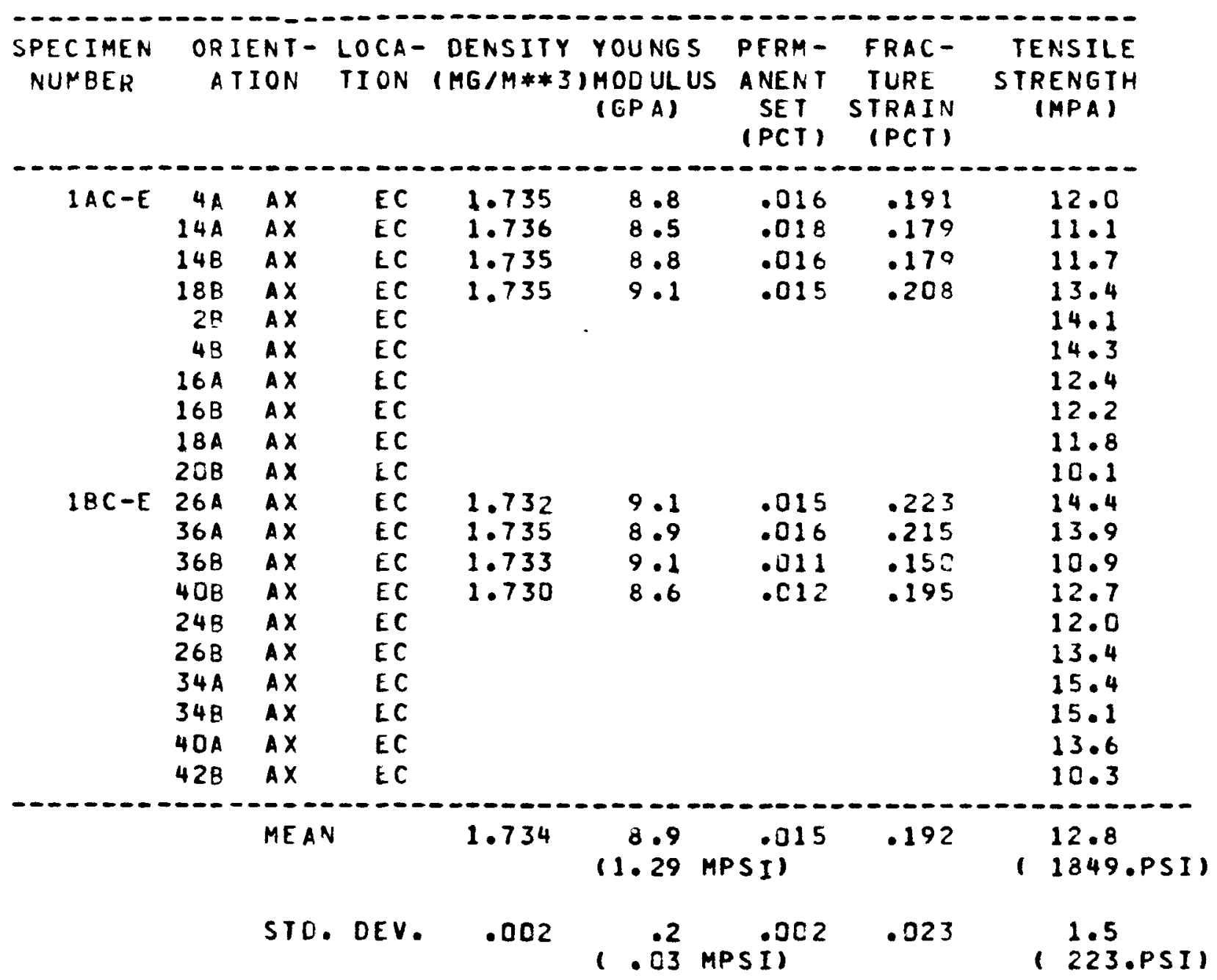


TABLE B-2 (Continued)

TENSILF PROPERTIES OF H-451 GRAPHITE

$\begin{array}{llll}\text { LOT NO.478 } & & \text { SPEC. DIA. } & 12.8 \mathrm{MM} \\ \text { LOG NO. } & 7194-52 & \text { SPEC. LENGTH } & 70 . \mathrm{MM}\end{array}$

LOG NO. TOG DENSITY

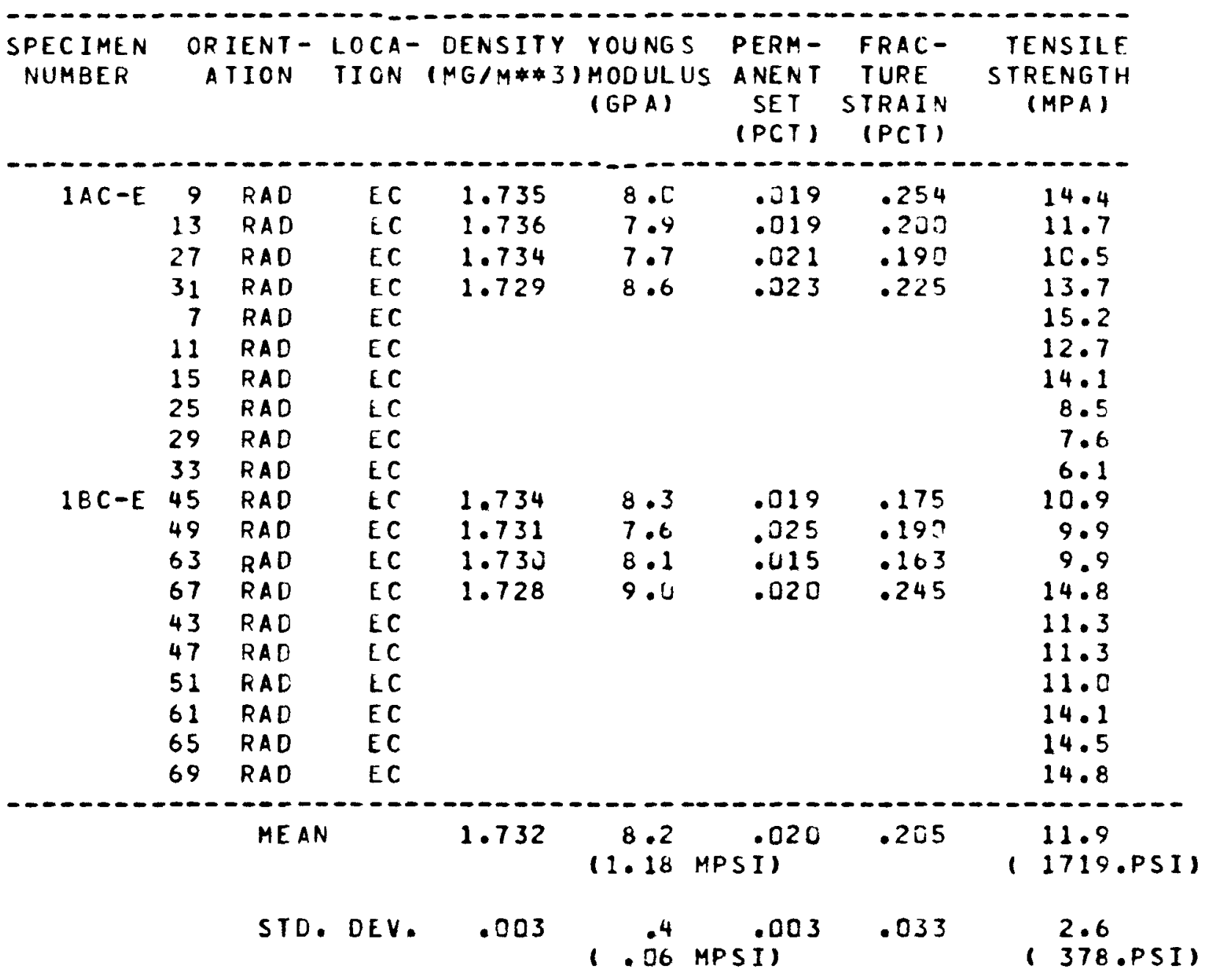


TABLE B-2 (Cont inued)

TENSILE PROPERTIES OF H-451 GRAPHITE

$\begin{array}{llll}\text { LOT NO.478 } & & \text { SPEC. DIA. } & 12.8 \mathrm{MM} \\ \text { LOG NO. } & 7194-52 & \text { SPEC. LENGTH } & 70 . \mathrm{MM}\end{array}$ LOG DENSITY _.. MGLM**3..

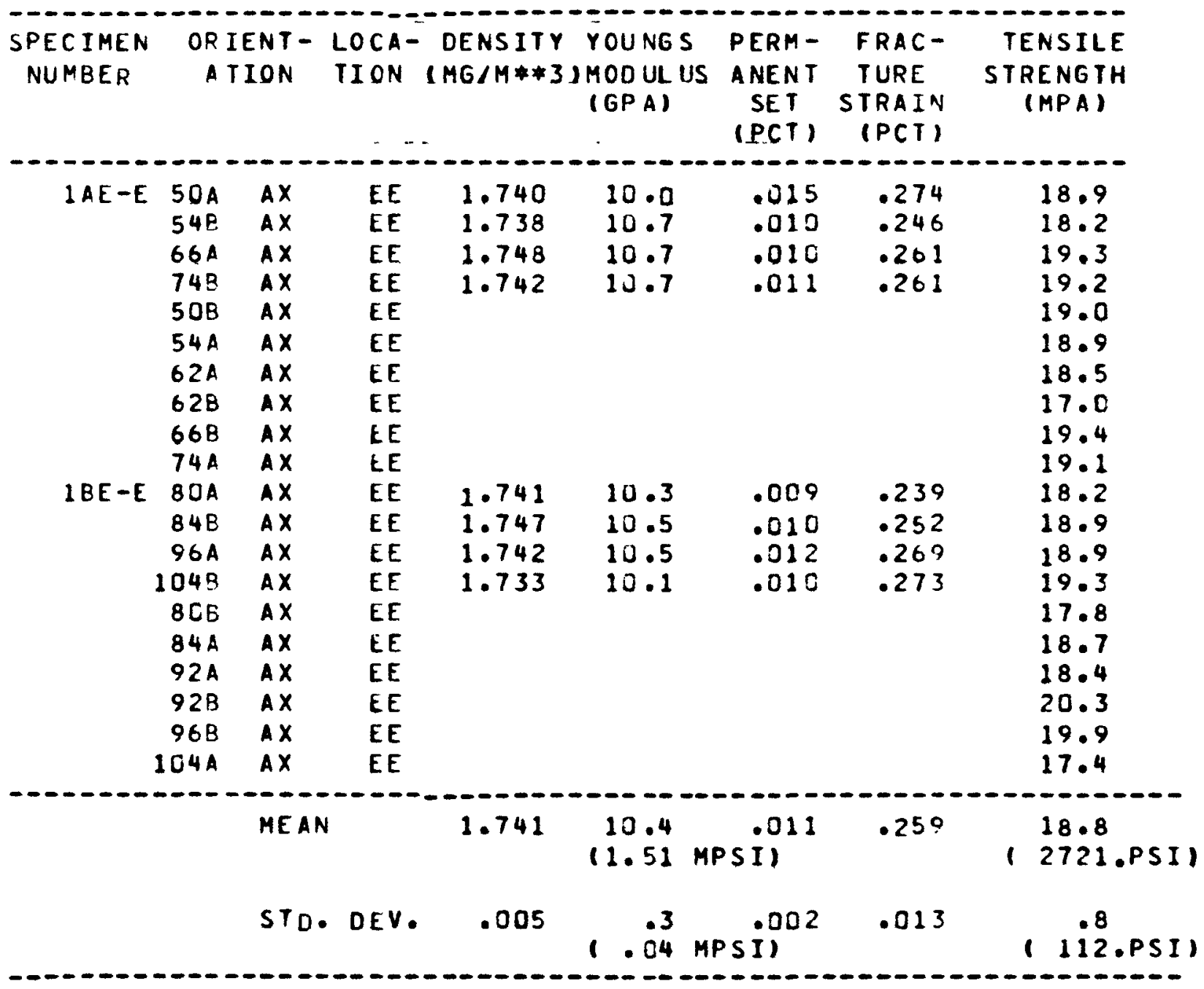


TABLE B-2 (Continued)

TENSILE PROPERTIES OF H-451 GRAPHITE

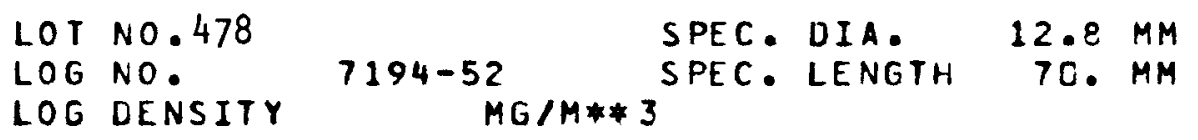

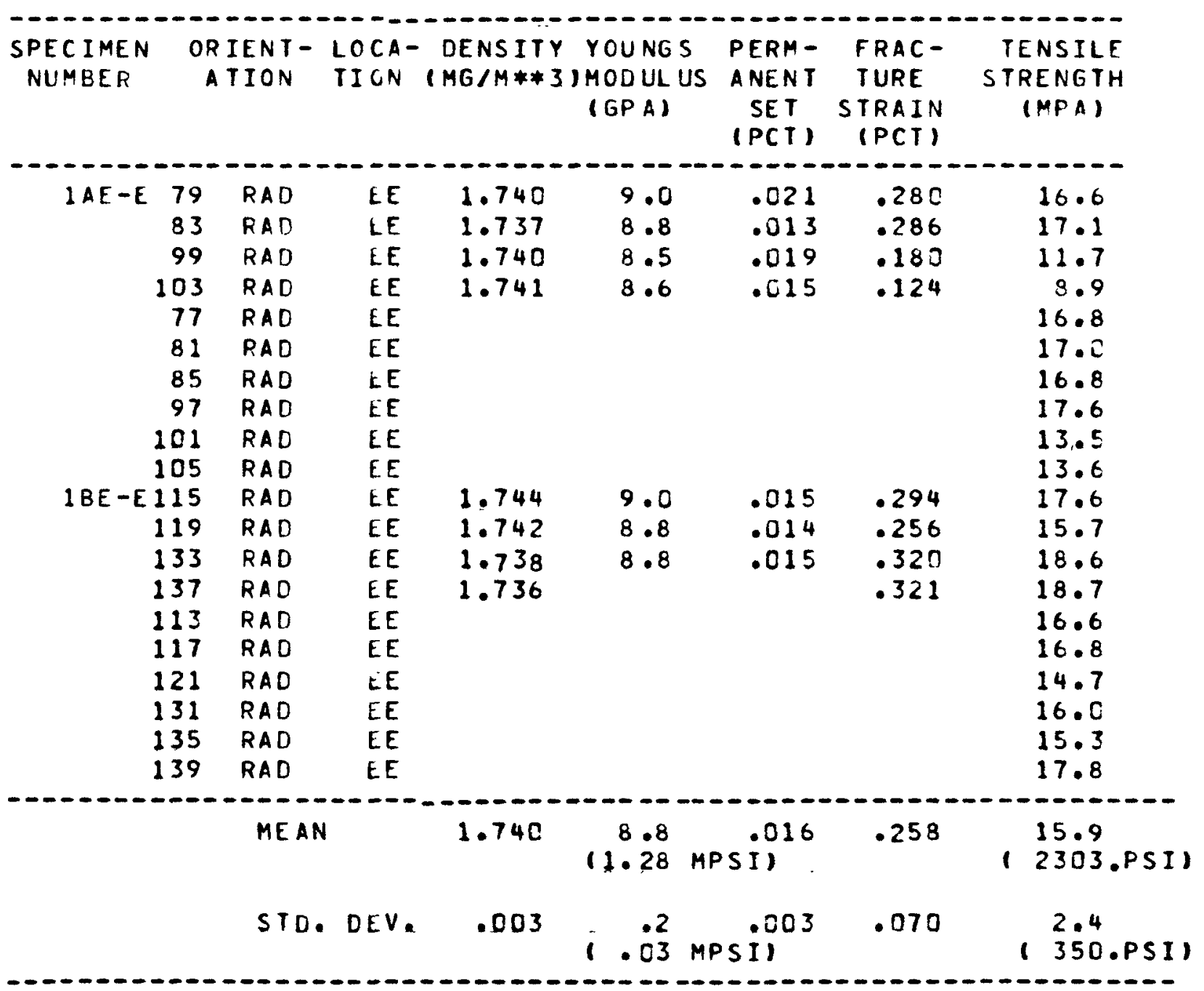


TABLE B-2 (Continued)

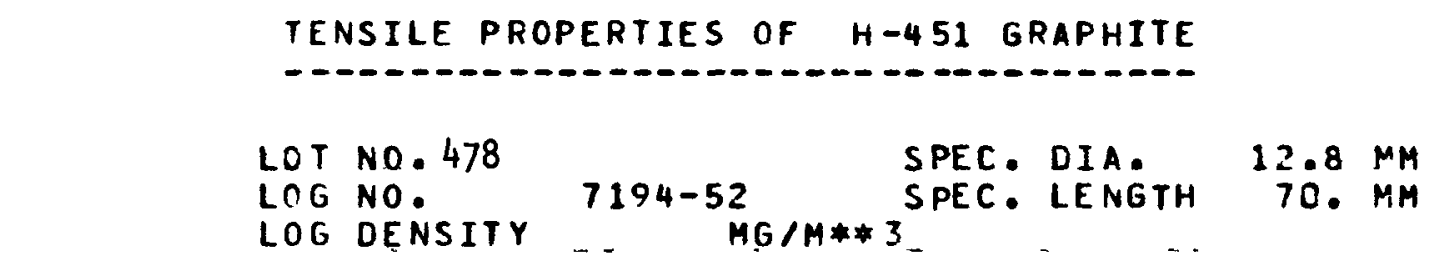

\begin{tabular}{|c|c|c|c|c|c|c|c|c|}
\hline $\begin{array}{l}\text { SPECIMEN } \\
\text { NUMBER }\end{array}$ & \multicolumn{2}{|c|}{$\begin{array}{l}\text { OR IENT- } \\
\text { ATION }\end{array}$} & $\begin{array}{l}\text { LOCA- } \\
\text { TION }\end{array}$ & $\begin{array}{l}\text { DENSITY } \\
\text { IMG/H* }\end{array}$ & $\begin{array}{l}\text { YOUNGS } \\
\text { IMODULUS } \\
\text { (GPA) }\end{array}$ & $\begin{array}{l}\text { PERM - } \\
\text { ANENT } \\
\text { SET } \\
\text { (PCT) }\end{array}$ & $\begin{array}{l}\text { FRAC- } \\
\text { TURE } \\
\text { STRAIN } \\
\text { (PCT) }\end{array}$ & $\begin{array}{l}\text { TENSILE } \\
\text { STRENGTH } \\
\text { (MPA) }\end{array}$ \\
\hline $3 B C-M$ & $\begin{array}{r}4 \mathrm{~A} \\
14 \mathrm{~A} \\
14 \mathrm{~B} \\
18 \mathrm{~B} \\
2 \mathrm{~B} \\
4 \mathrm{~B} \\
12 \mathrm{~A} \\
12 \mathrm{~B} \\
18 \mathrm{~A} \\
20 \mathrm{~B} \\
26 \mathrm{~A} \\
36 \mathrm{~A} \\
36 \mathrm{~B} \\
4 \mathrm{CB} \\
24 \mathrm{~B} \\
26 \mathrm{~B} \\
38 \mathrm{~A} \\
38 \mathrm{~B} \\
40 \mathrm{~A} \\
42 \mathrm{~B}\end{array}$ & $\begin{array}{l}A X \\
A X \\
A X \\
A X \\
A X \\
A X \\
A X \\
A X \\
A X \\
A X \\
A X \\
A X \\
A X \\
A X \\
A X \\
A X \\
A X \\
A X \\
A X \\
A X\end{array}$ & $\begin{array}{l}M L C \\
M L C \\
M L C \\
M L C \\
M L C \\
M L C \\
M L C \\
M L C \\
M L C \\
M L C \\
M L C \\
M L C \\
M L C \\
M L C \\
M L C \\
M L C \\
M L C \\
M L C \\
M L C \\
M L C\end{array}$ & $\begin{array}{l}1.731 \\
1.726 \\
1.720 \\
1.718\end{array}$ & $\begin{array}{r}10.0 \\
9.8 \\
9.4 \\
9.8\end{array}$ & $\begin{array}{l}.015 \\
.011 \\
.010 \\
.016\end{array}$ & $\begin{array}{l}.244 \\
.189 \\
.215 \\
.222\end{array}$ & $\begin{array}{l}16.7 \\
13.7 \\
15.1 \\
15.5 \\
17.5 \\
16.4 \\
15.8 \\
14.9 \\
15.4 \\
15.0 \\
15.5 \\
15.1 \\
14.7 \\
16.2 \\
14.2 \\
14.8 \\
15.9 \\
15.8 \\
15.8 \\
16.1\end{array}$ \\
\hline & & MEAI & - & 1.724 & $\begin{array}{r}9.7 \\
11.41 \mathrm{M}\end{array}$ & PSII & .221 & $\begin{array}{l}15.5 \\
12248\end{array}$ \\
\hline & & STD & DEV. & .004 & $1.04 \mathrm{~m}$ & PSII & .018 & $1 \quad 126$ \\
\hline
\end{tabular}


TABLE B-2 (Cont inued)

TENSILE PFOPERTIES OF H-451 GRAPHITE

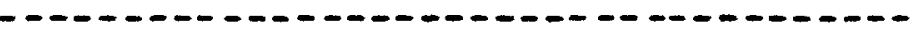

\begin{tabular}{|c|c|c|c|c|c|}
\hline $\begin{array}{l}\operatorname{LOT} \\
\operatorname{LOG}\end{array}$ & $\begin{array}{l}\text { NC. } 478 \\
\text { NC. }\end{array}$ & $7194-52$ & $\begin{array}{l}\text { SPEC. } \\
\text { SPEC. }\end{array}$ & $\begin{array}{l}\text { DIA. } \\
\text { LENGTH }\end{array}$ & $\begin{array}{c}12.8 \\
70 .\end{array}$ \\
\hline 0 & DENSITY & & & & \\
\hline
\end{tabular}

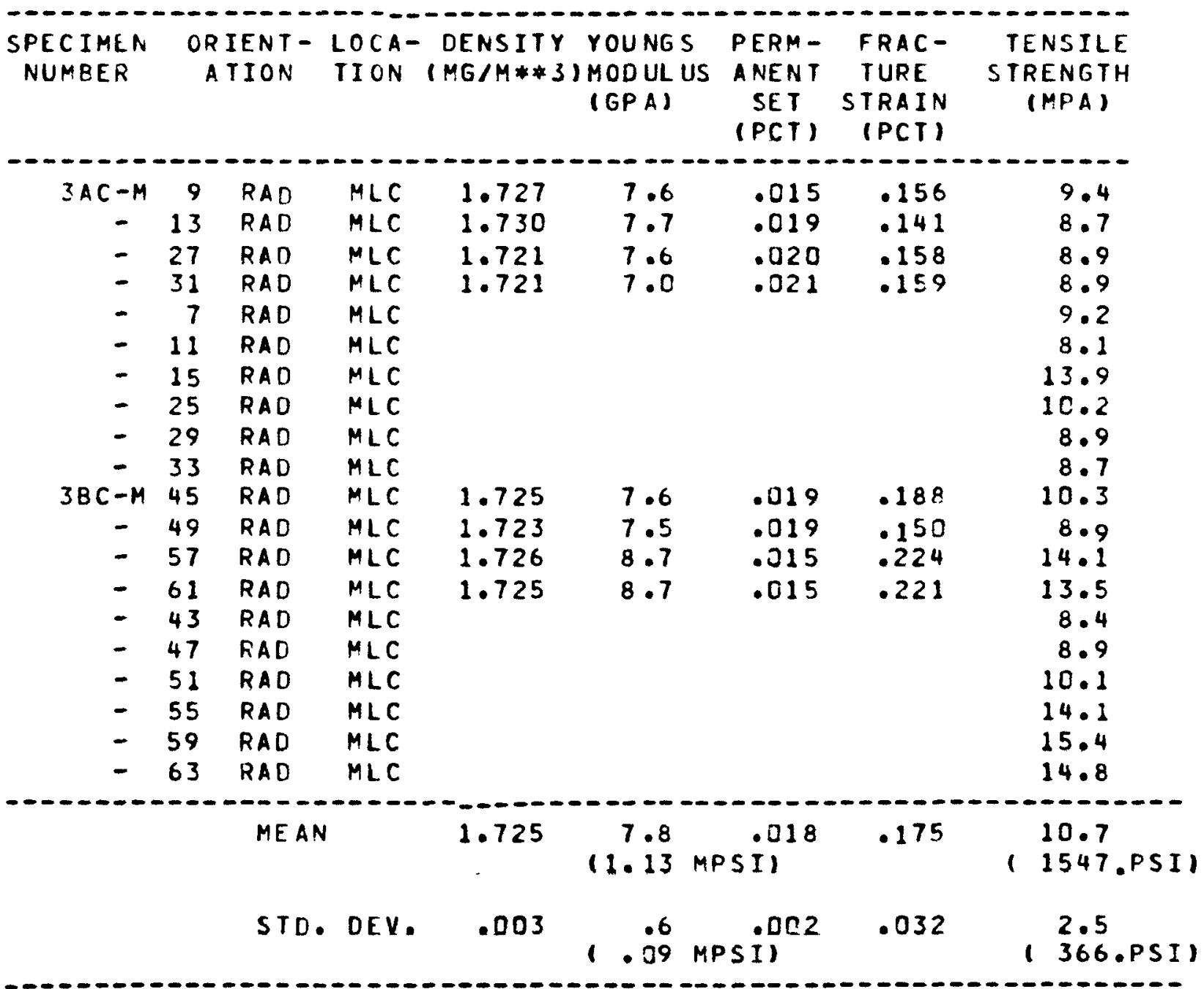


TABLE B-2 (Continued)

TENSILE PFOPERTIES OF H-451 GRAPHITE

TENSILE PROPERTIES OF

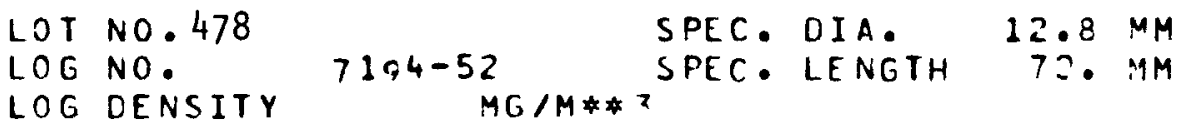

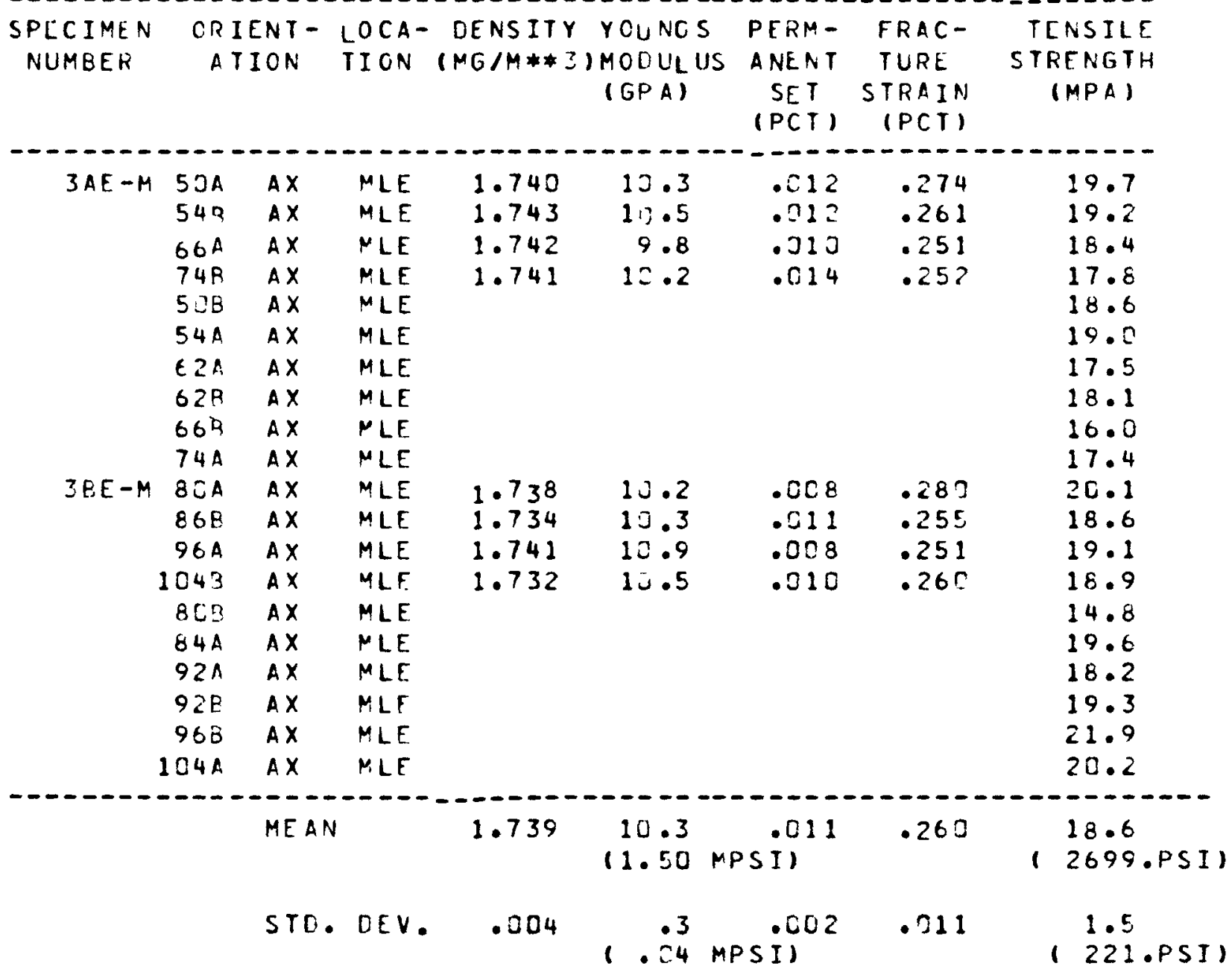


TABLE B-2 (Continued)

TENSILE PFOPERTIES OF H-451 GRAPHITE

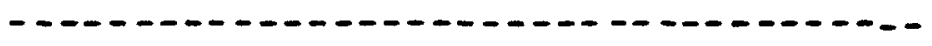

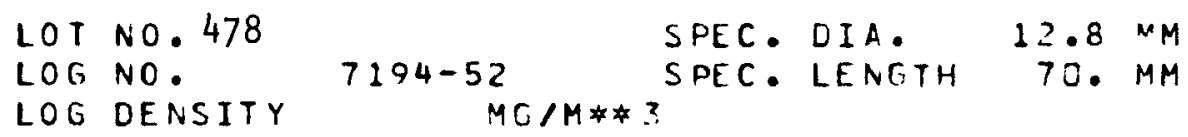

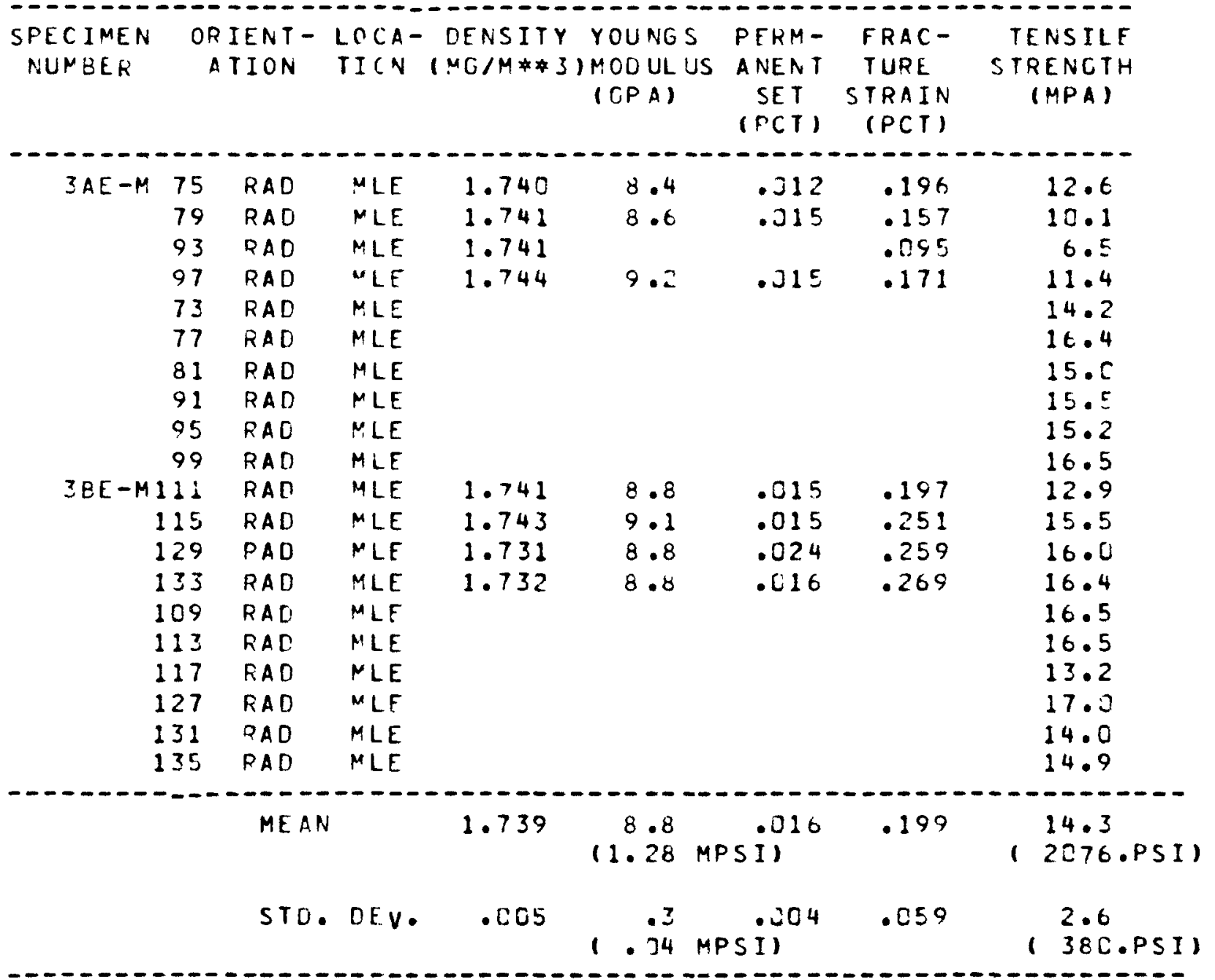


TABLE B-2 (Continued)

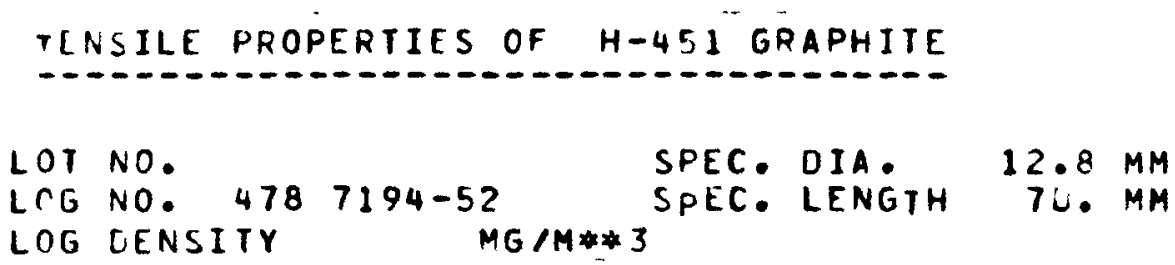

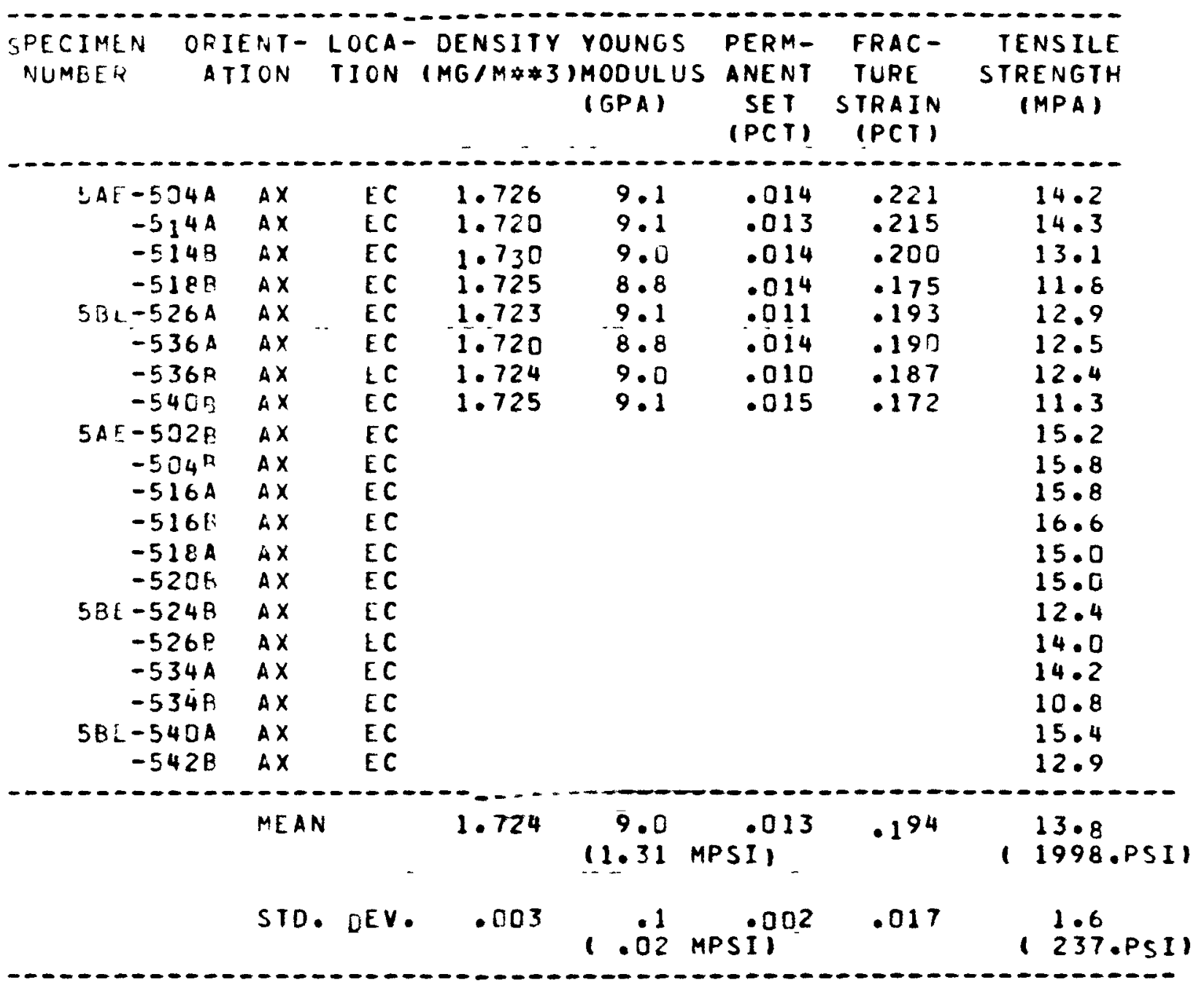


TABLE B-2 (Continued)

TENSILE PROPERTIES OF H-4E1 GRAPHITE
LOT NO.
LOG NO. $4787194-52$
LOG OENSITY

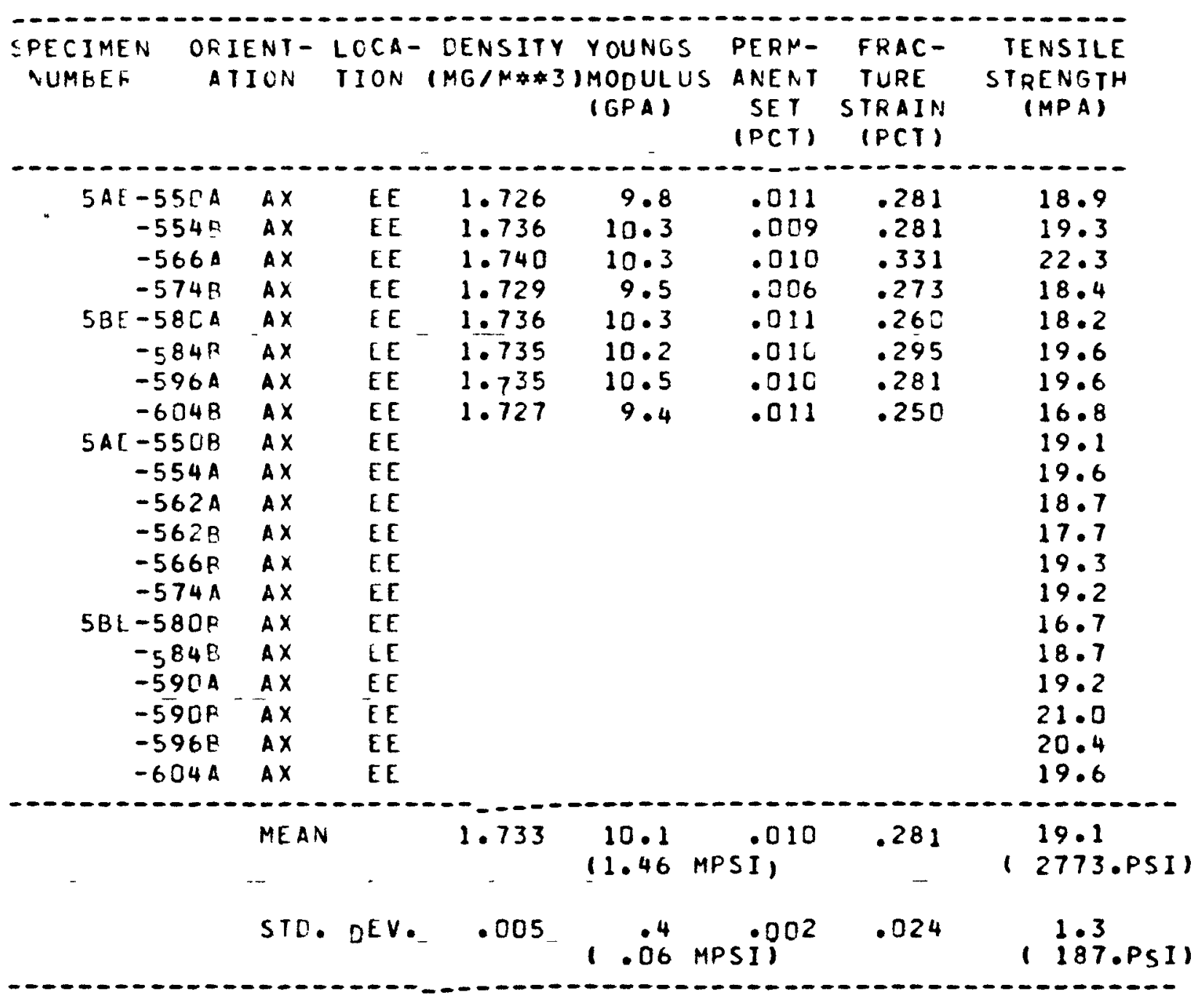


TABLE B-2 (Continued)

TENSILE PROPERTIES OF H-451 GRAPHITE
LOT NO.
LOG NO. $4787194-52 \quad$ SPEC. DIA.
LOC DENSITY

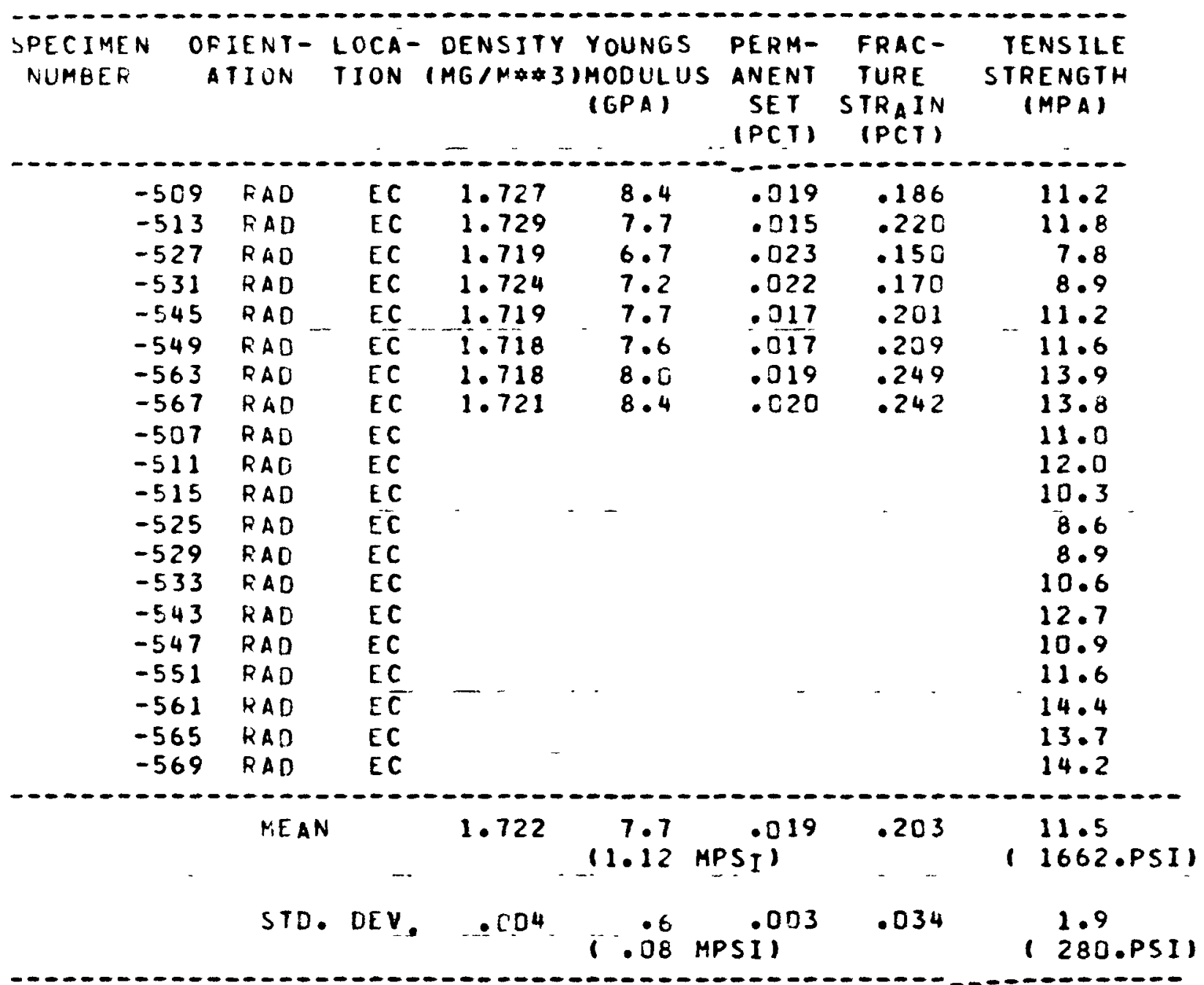


TABLE B-2 (Continued)

TENSILE PROPERTILS OF H-451 GRAPHITE -..
LOT NG.
SPEC. DIA.
LOG NO. $4787194-52$
SPEC. LENGTH
$12.8 M M$
LOG OENSITY
$M G / M * 3$
70. MM

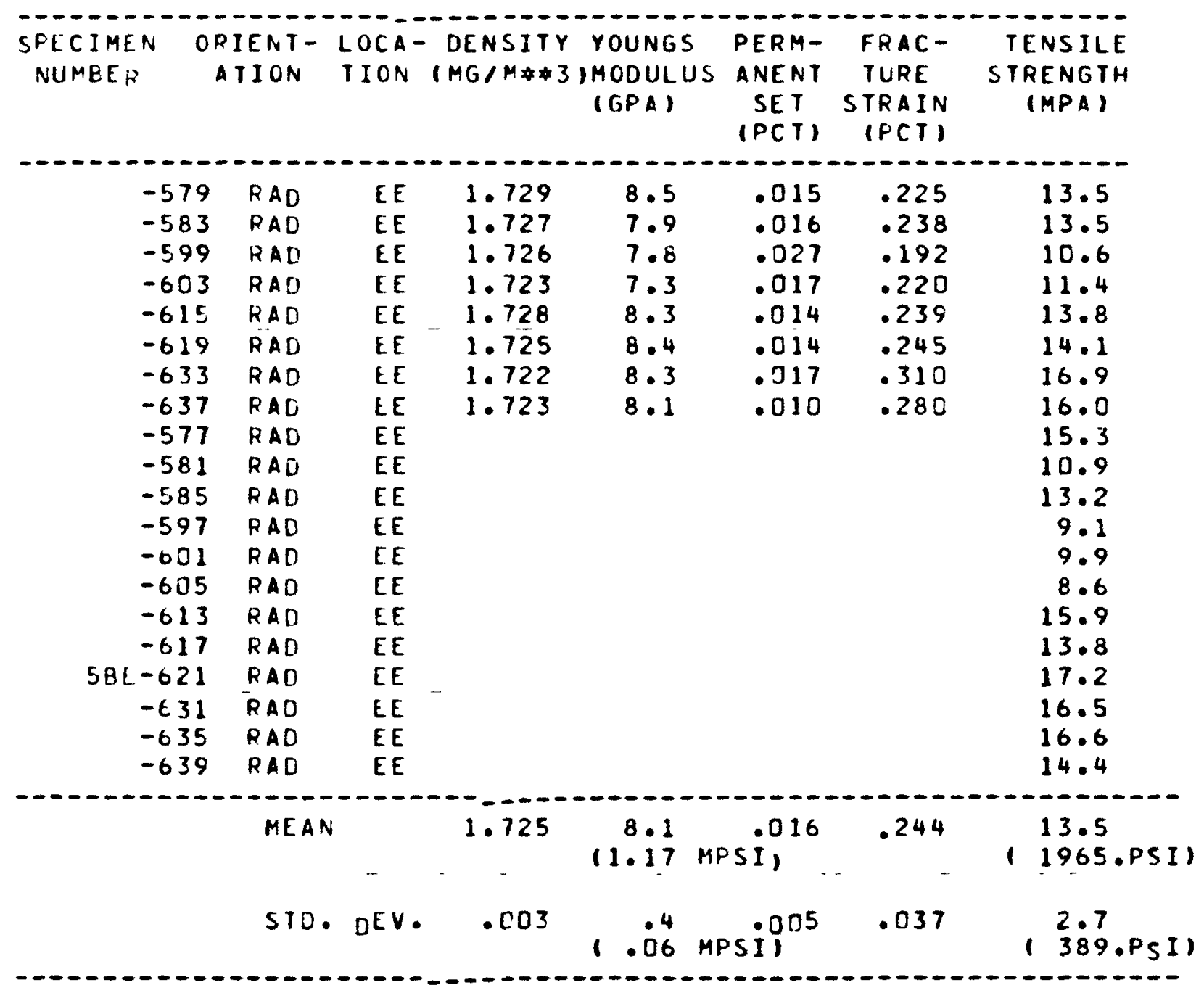


TABLE B-3

TENSILE PROPERTIES CF H-45i GRAPHITE

LOT NO. 478
LOG NO.
LOG DENSITY

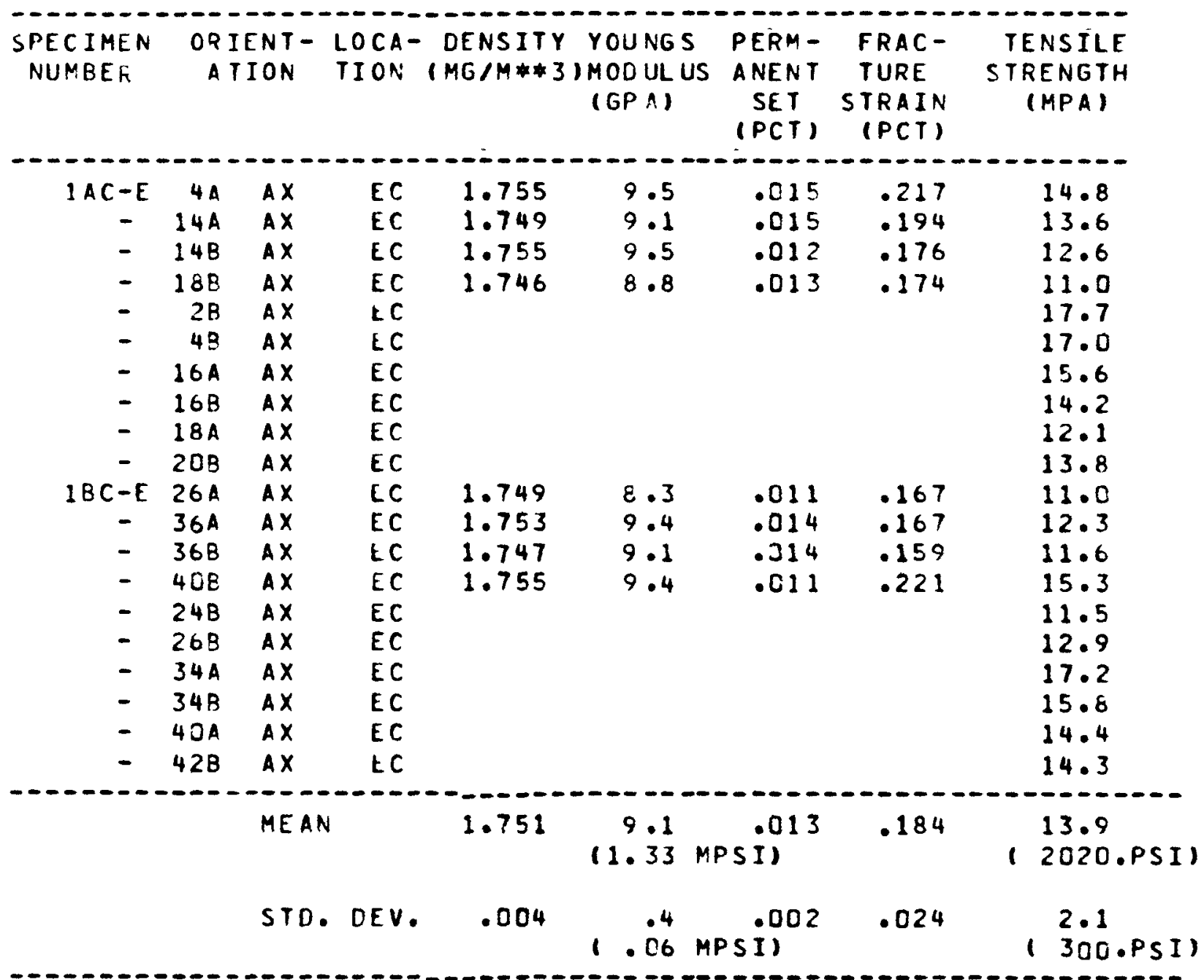


TABLE B-3 (Continued)

TENSILE PROPERTIES OF H-451 GRAPHITE

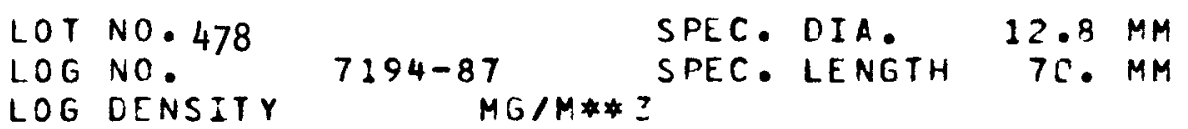

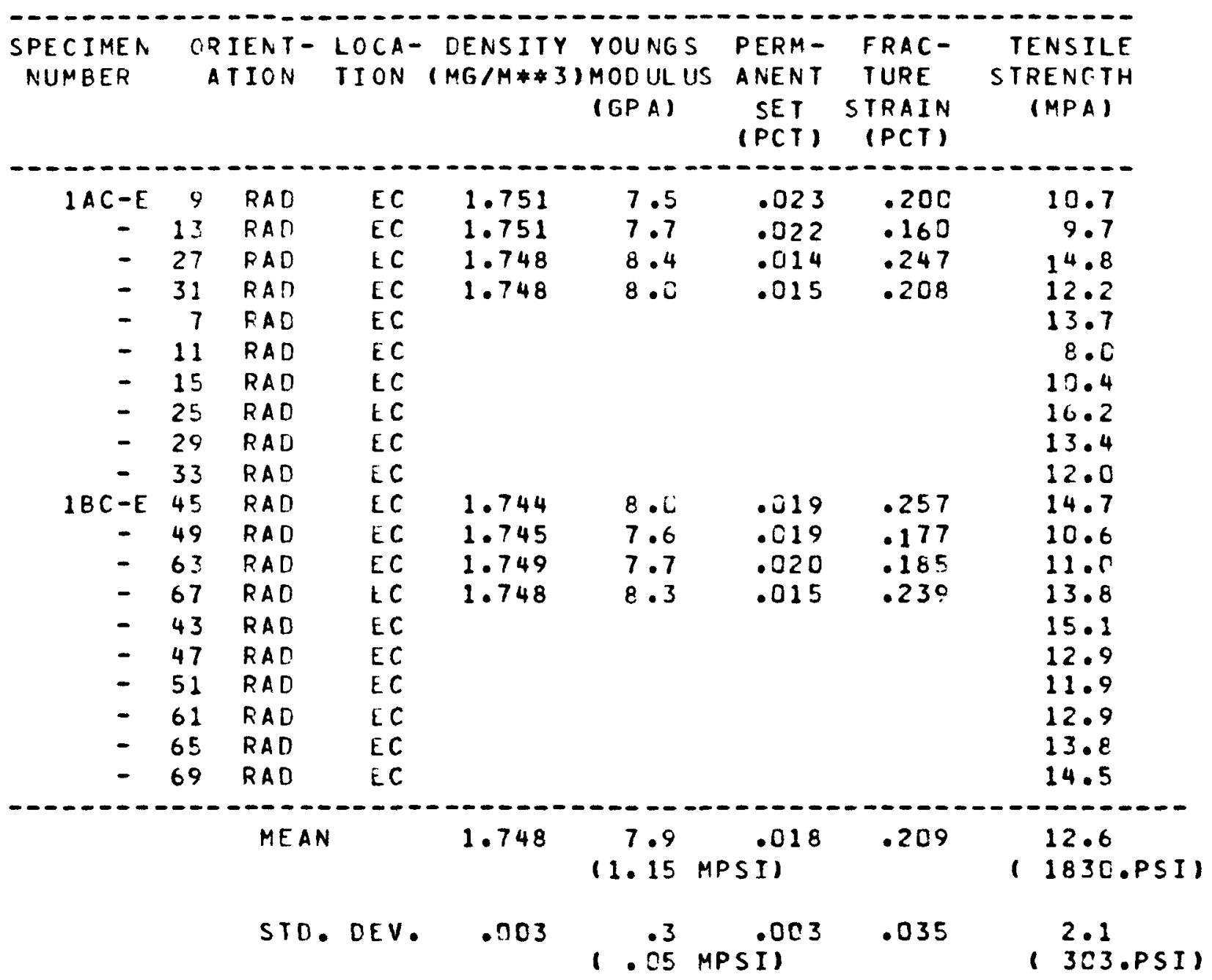


TABLE B-3 (Continued)

TENSILE PROPERTIES OF H-45் GRAPHITE

- .
$\operatorname{LOT} N O \cdot 478$
LOG NO.
SPEC. DIA.
$12.8 \mathrm{MM}$
LOC DENSITY
$7194-87$
$M G / M * 3$
SPEC. LENGTH 70. MM

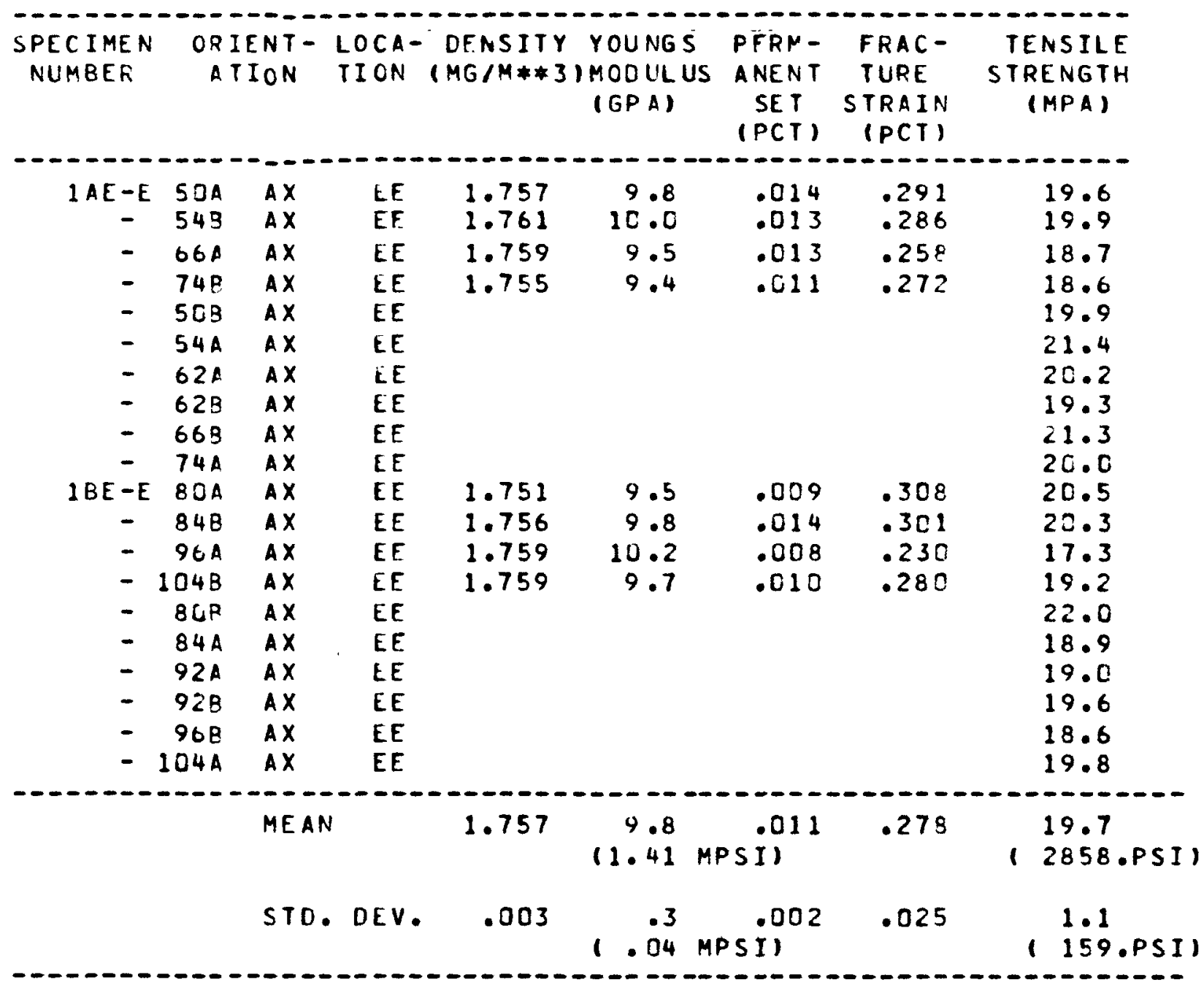


TABLE B-3 (Cont inued)

TENSILE PROPERTIES OF H-451 GRAPHITE

- - - - - - - - - - - - - - - - - - - - - - -

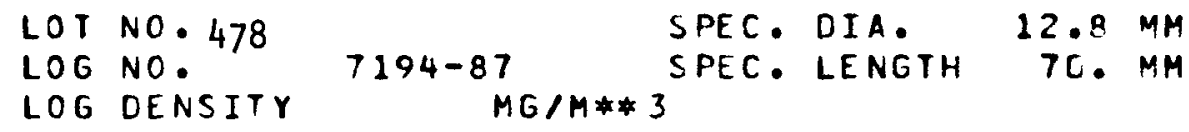

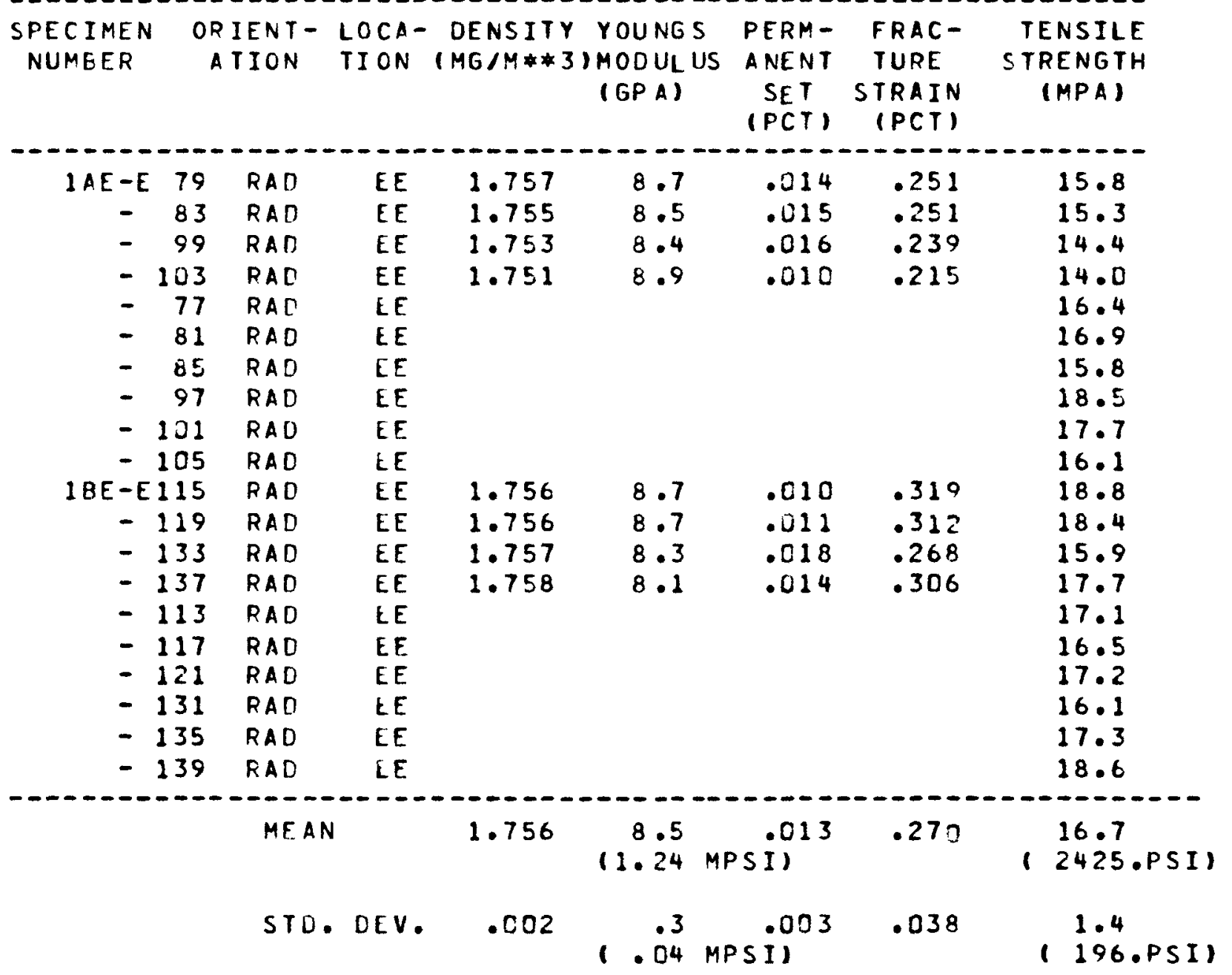


TABLE B-3 (Continued)

TENSILE PROPERTIES OF H-451 GPAPHITE

TENSILE PROPERTIES OF H

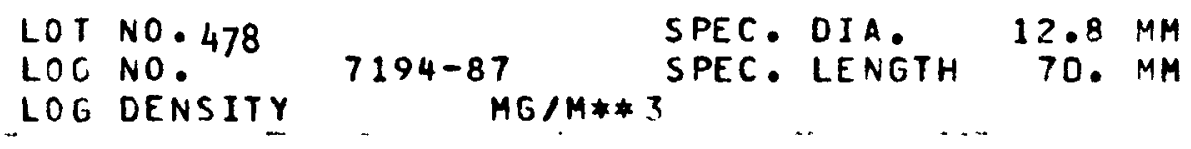

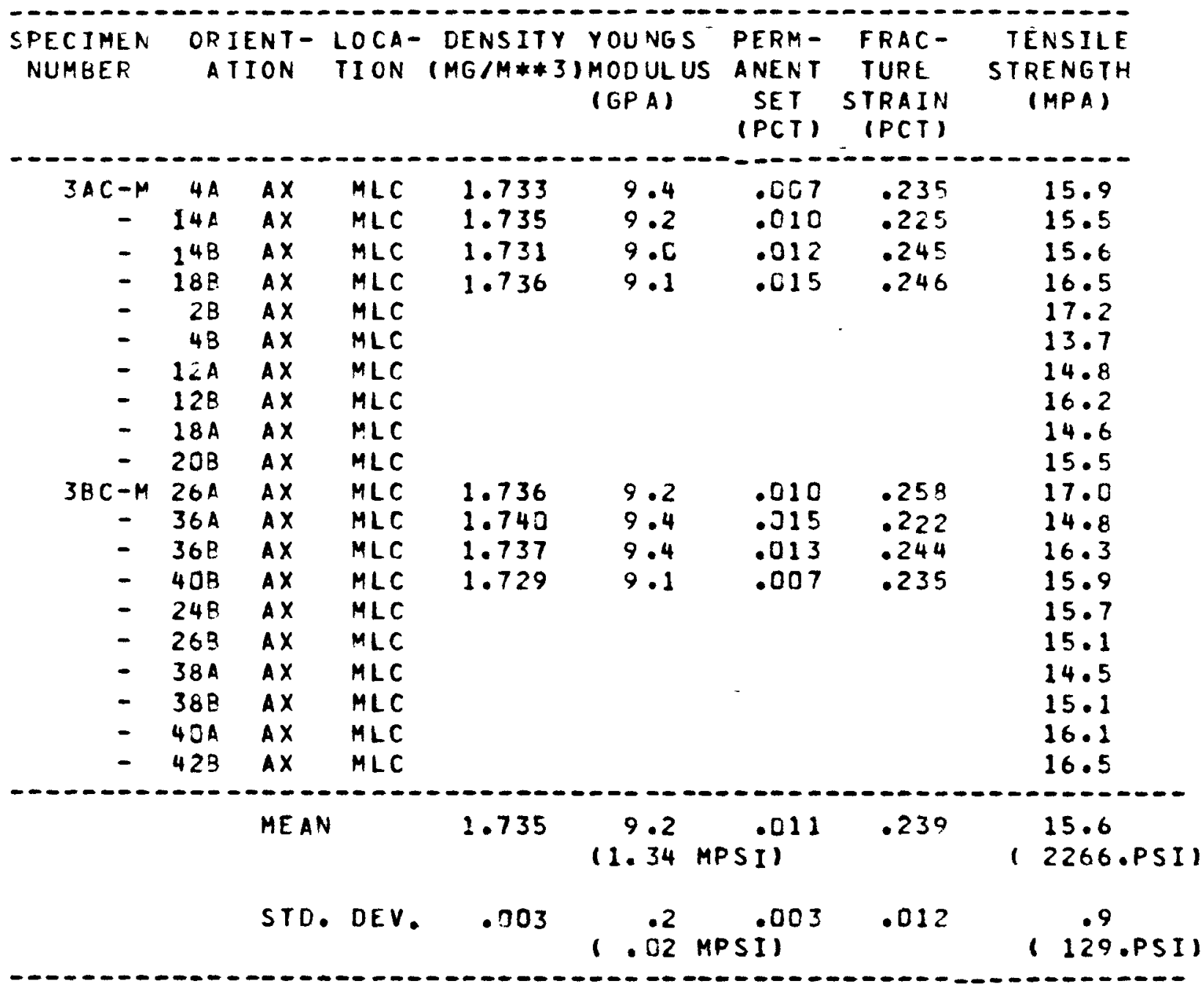


TABLE B-3 (Continued)

TENSILE PROPERTIES OF H-451 GRAPHITE

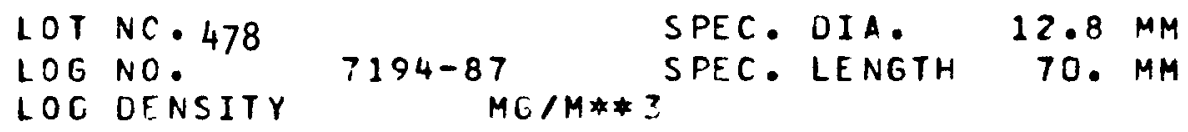

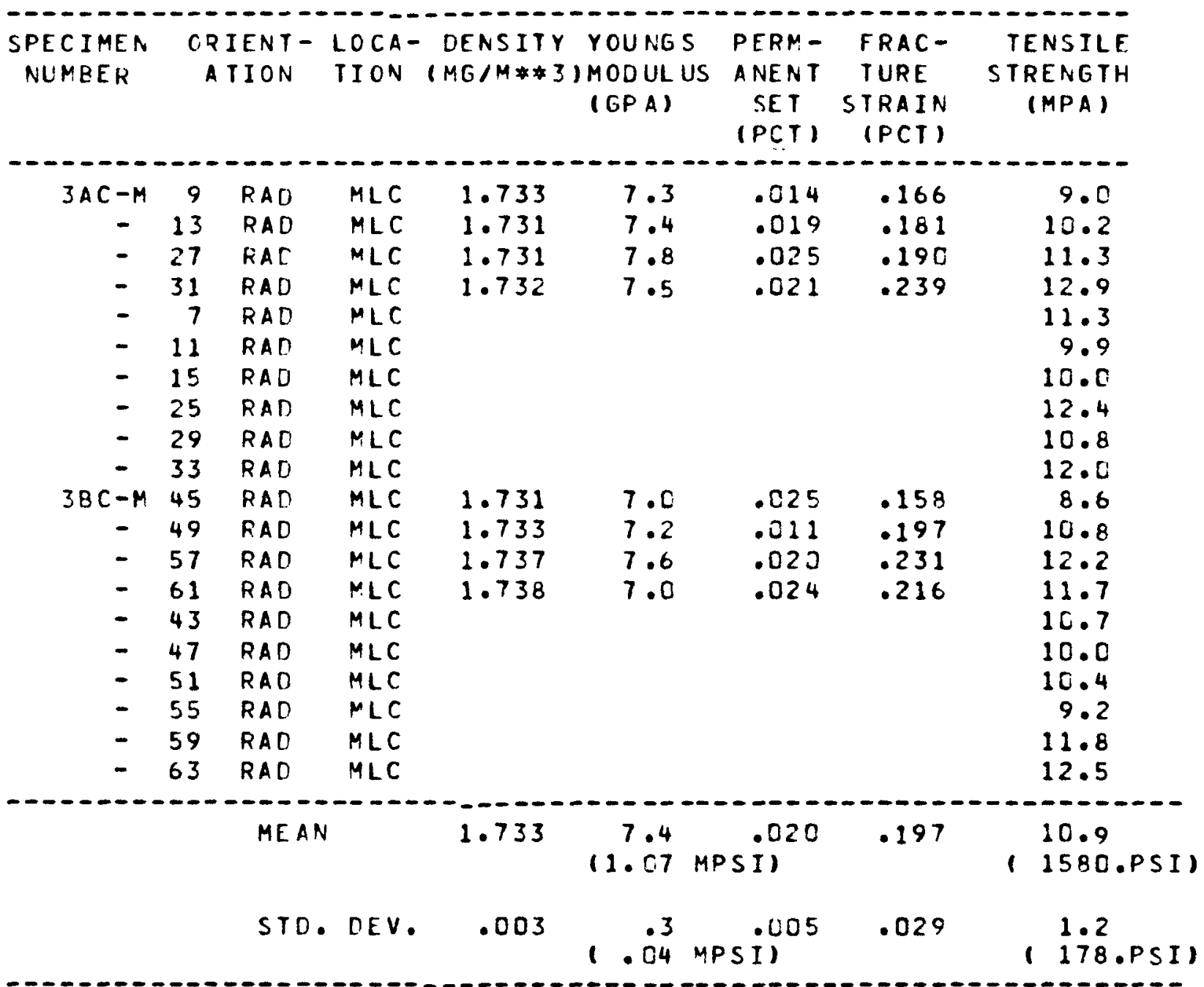


TABLE B-3 (Cont inued)

TENSILE PROPERTIES OF H-451 GRAPHITE
LOT NO. 478
LOG NO.
$\begin{array}{ll}\text { SPEC. DIA. } & 12.8 \mathrm{MM} \\ \text { SPEC. LENGTH } & 7 . \text { MM }\end{array}$
LOC DIENSITY
$7194-87$ $M G / M * * 3$

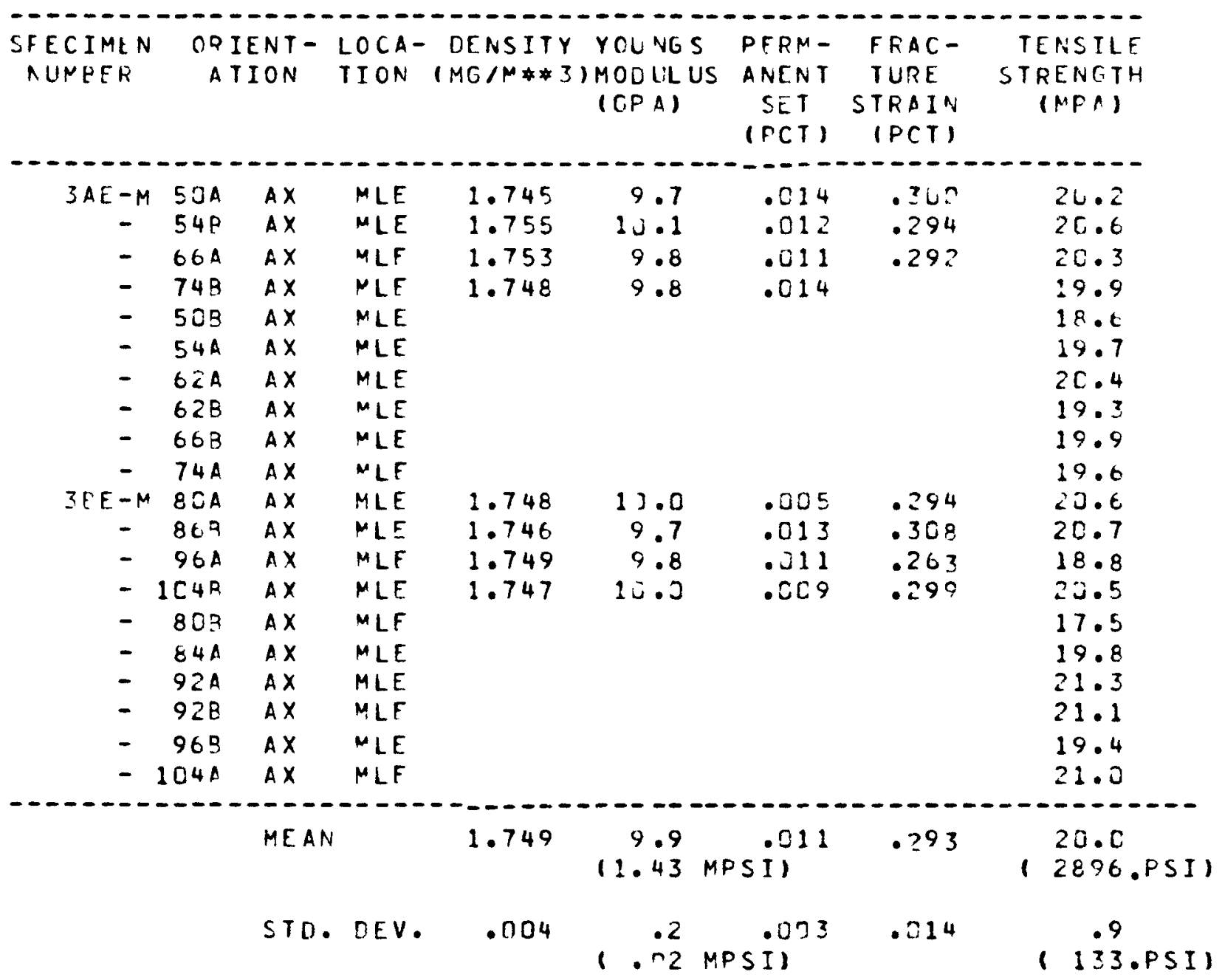


TABLE B-3 (Continued)

TENSILE PFOPERTIES OF H-451 GRAPHITE

LOT NO. 478
LOC NO. SPEC. DIA.

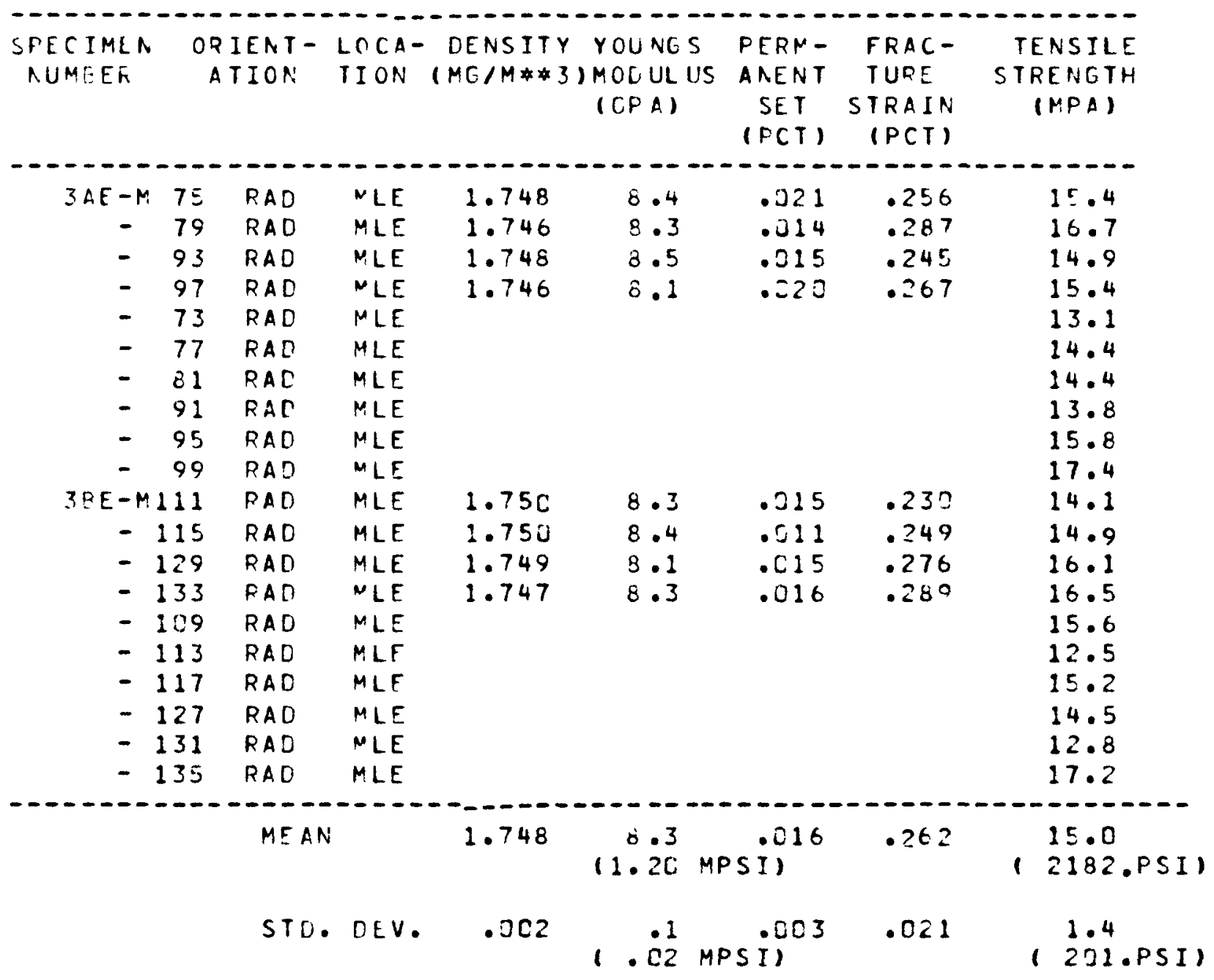


TABLE B-4

FLFXIIRAL PROPERTIFS OF H-45I GRAPHITF

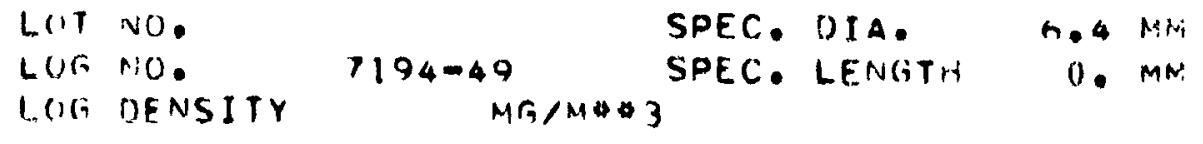

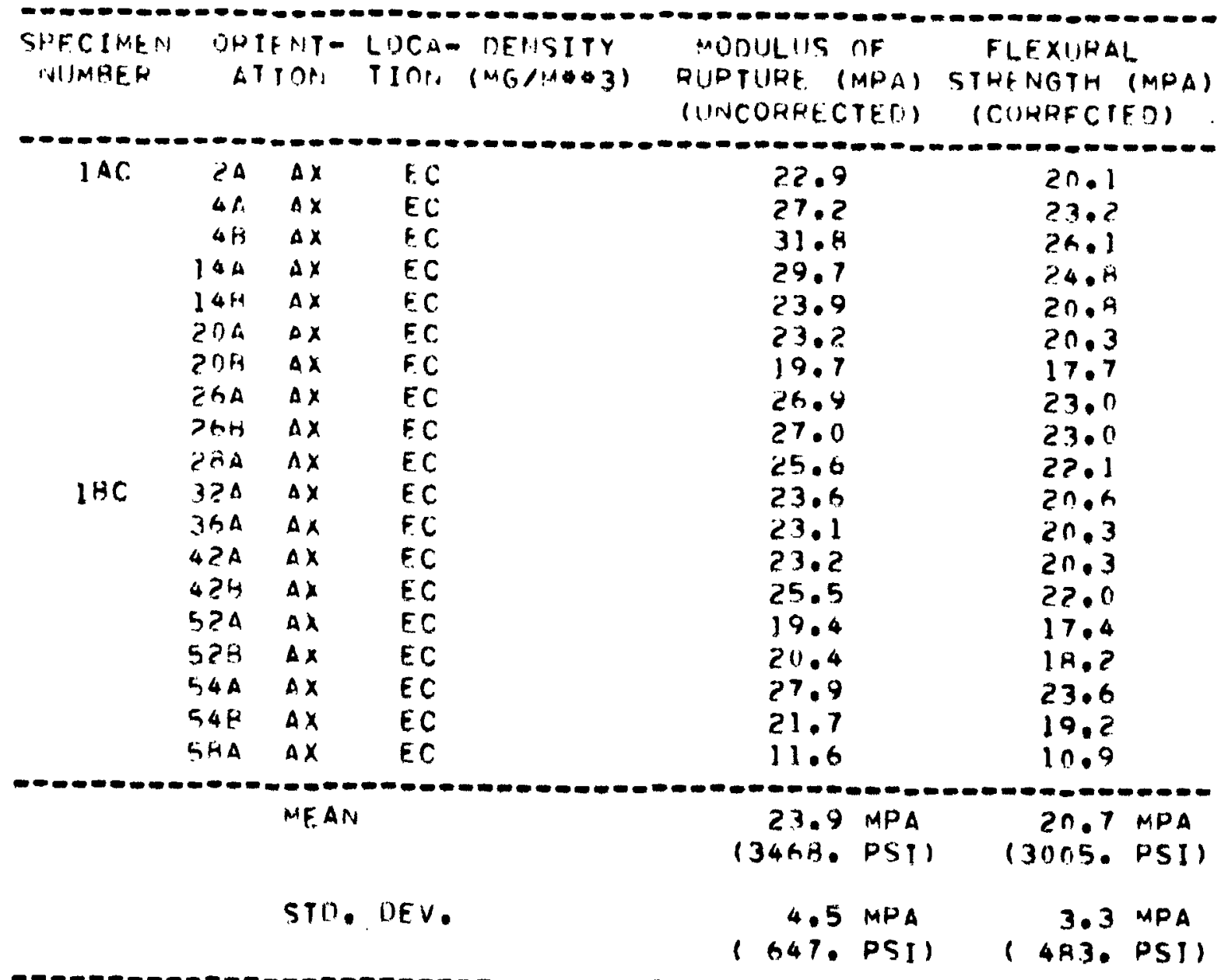


TABLE B-4 (Continued)

FLEXIIRAL PROPEFTIES OF H-45I GHAPHITE

- - - - - - - - - - - - - - - - - - - - - - - - - - - - - - - - - -

LOI NO. SPEC. DIA. $\quad 0.4 \mathrm{MM}$

Lor. AR.

LOG DENSITY

7194-49 SPEC. LENGTH O. MM $M G / M * 3$

\begin{tabular}{|c|c|c|c|c|c|c|c|}
\hline $\begin{array}{l}\text { SPFCIMEN } \\
\text { NIIMOER }\end{array}$ & \multicolumn{2}{|c|}{$\begin{array}{l}\text { ORIEINT- } \\
\text { ATIDT. }\end{array}$} & $\begin{array}{l}\text { LOCA- DENSITY } \\
\text { TIOIN (MG/M*3) }\end{array}$ & $\begin{array}{l}\text { MODULUS } \\
\text { HUPTURE } \\
\text { IUNCORREC }\end{array}$ & $\begin{array}{l}\text { OF } \\
\text { (MPA) } \\
\text { CTED) }\end{array}$ & $\begin{array}{l}\text { FLEXIIRAL } \\
\text { STRENGTH } \\
\text { ICORECTE }\end{array}$ & $\begin{array}{l}L \\
(M P A) \\
E D)\end{array}$ \\
\hline $\begin{array}{l}1 A C \\
1 A C\end{array}$ & $\begin{array}{l}1 \\
5 \\
7 \\
11 \\
11 \\
23 \\
25 \\
29 \\
31 \\
35 \\
41 \\
45 \\
47 \\
51 \\
53 \\
63 \\
65 \\
69 \\
71 \\
75\end{array}$ & 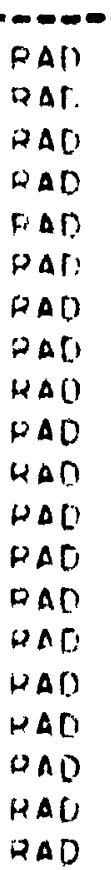 & $\begin{array}{l}E C \\
E C \\
E C \\
E C \\
E C \\
E C \\
E C \\
E C \\
E C \\
E C \\
E C \\
E C \\
E C \\
E C \\
E C \\
E C \\
E C \\
E C \\
E C \\
E C\end{array}$ & $\begin{array}{l}28.7 \\
24.2 \\
22.3 \\
23.9 \\
21.4 \\
23.7 \\
24.9 \\
23.7 \\
23.4 \\
23.2 \\
29.6 \\
28.9 \\
29.2 \\
22.8 \\
25.4 \\
29.6 \\
29.2 \\
2.4 .4 \\
25.4 \\
20.4\end{array}$ & $\begin{array}{l}7 \\
2 \\
3 \\
9 \\
4 \\
7 \\
9 \\
7 \\
4 \\
2 \\
6 \\
9 \\
2 \\
0 \\
4 \\
6 \\
2 \\
4 \\
4 \\
4\end{array}$ & $\begin{array}{l}23.6 \\
20.7 \\
19.4 \\
20.5 \\
19.8 \\
20.4 \\
21.2 \\
20.4 \\
20.2 \\
20.0 \\
24.2 \\
23.8 \\
24.0 \\
19.7 \\
21.5 \\
24.2 \\
23.9 \\
20.9 \\
21.6 \\
18.0\end{array}$ & $-\infty$ \\
\hline & & MEA & & $\begin{array}{r}25.2 \\
13658\end{array}$ & $\begin{array}{l}\text { MPA } \\
\text { PSTI }\end{array}$ & $\begin{array}{r}21.4 \\
13098 .\end{array}$ & $\begin{array}{l}\text { MPA } \\
\text { PSII }\end{array}$ \\
\hline & & ST & DEV. & $\begin{array}{r}2.9 \\
1425 .\end{array}$ & $\begin{array}{l}\text { MPA } \\
\text { PSII }\end{array}$ & $\begin{array}{r}1.9 \\
1280 .\end{array}$ & $\begin{array}{l}\text { MPA } \\
\text { PSI) }\end{array}$ \\
\hline
\end{tabular}


TABLE B-4 (Continued)

FLFXIIKAL PRCPERTIES UF H-45I GRAPHTTE

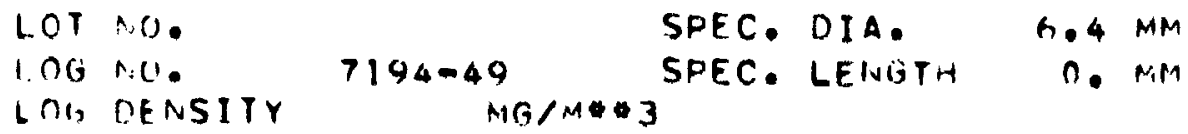

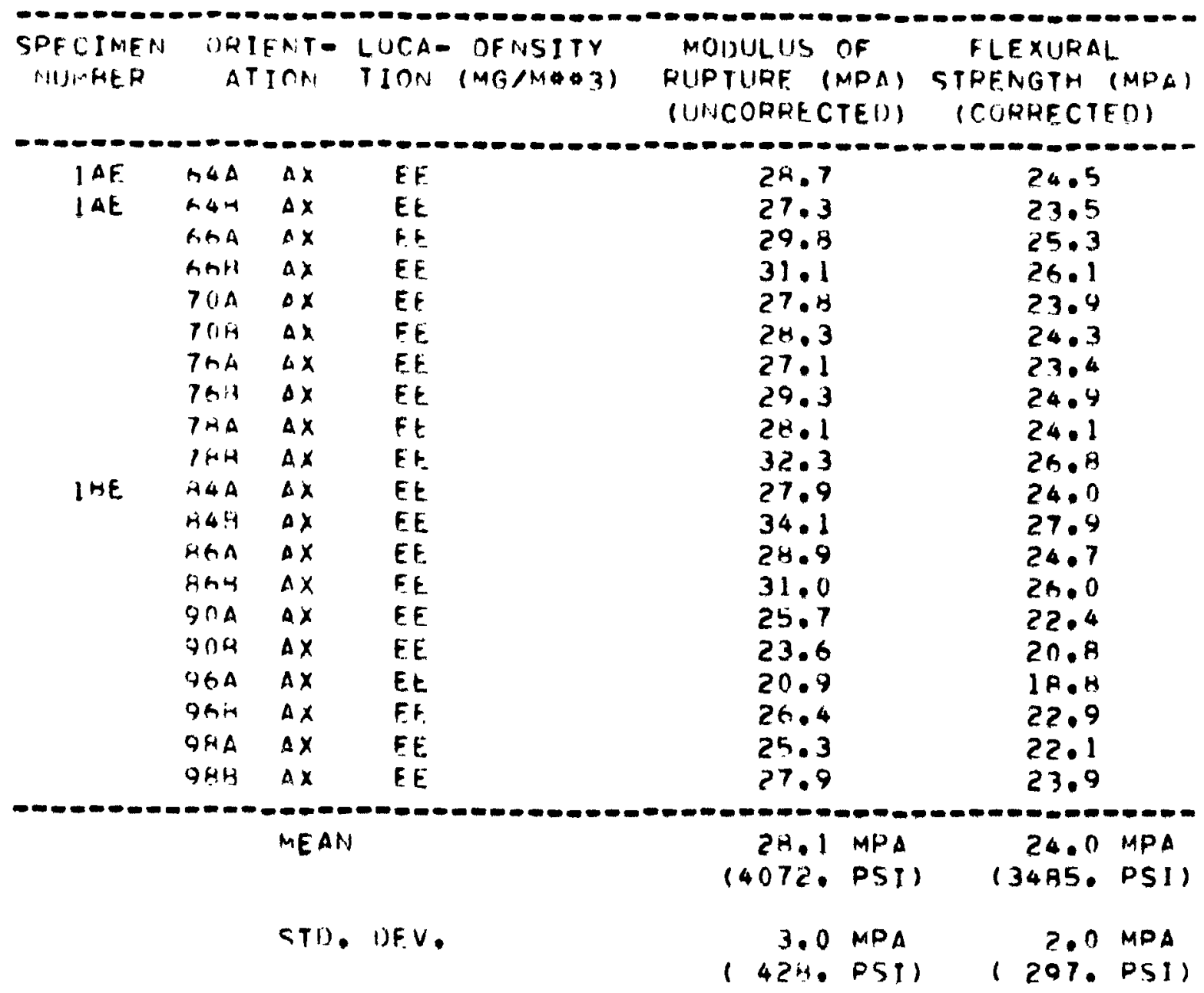


TABLE B-4 (Continued)

FLEXIHAL PHOPERTIFS OF Ha451 GRAPHITE

- - - - - - - - - - - - - - - - - - - - - - - - - - - - - - - -
LOT NOB.
SPE.C. DIA.
LOC. MO.
LOG UEASITY
$7194-49$
SPEC. LFNGTH
6.4 MM $M G / M \$ 3$

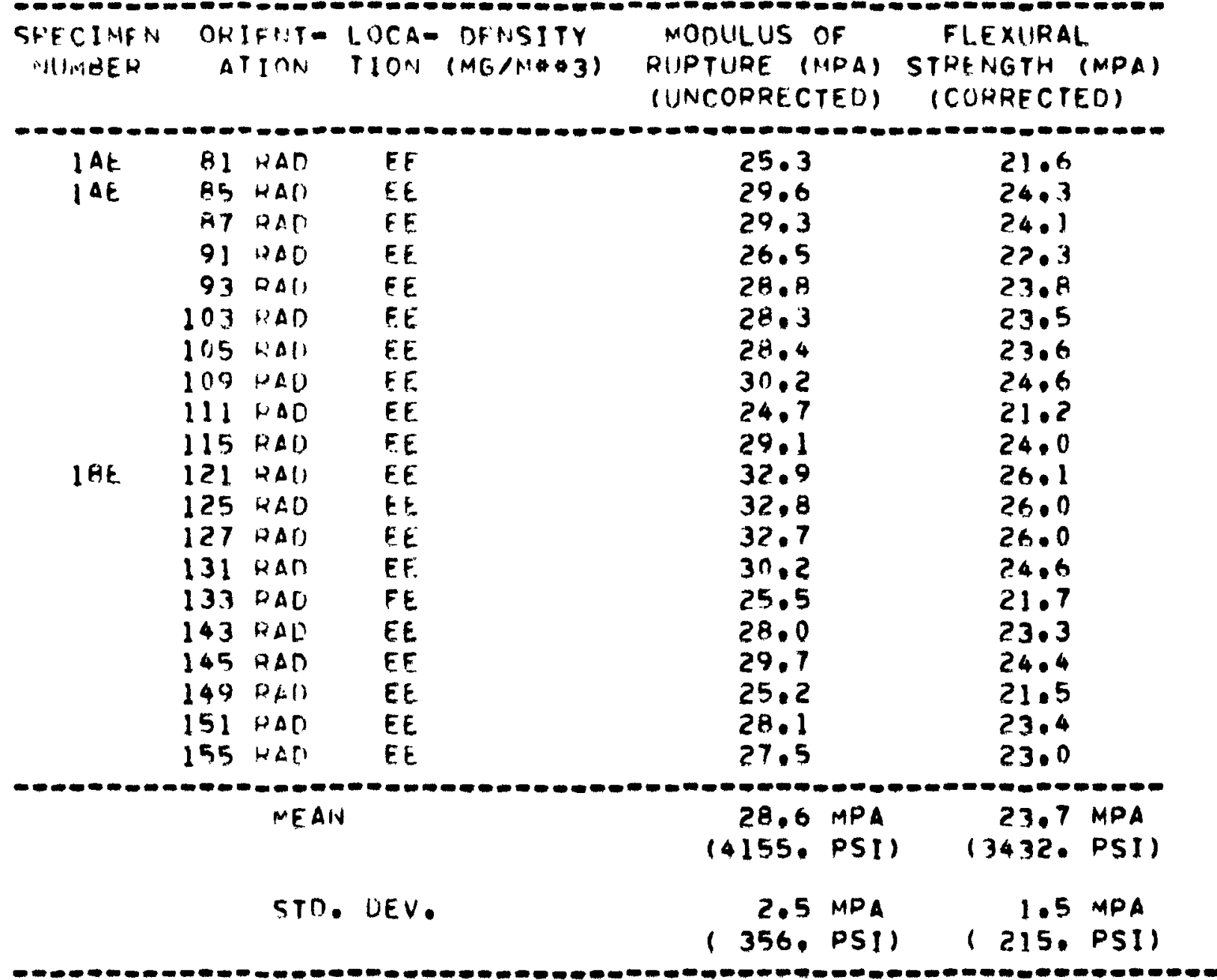


TABLE B-4 (Continued)

FLEXIIRAL PHOPERTIES OF H=45I GRAPHITF

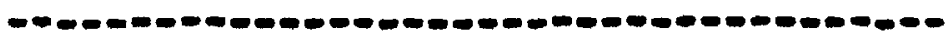

LUT NO.
LOG MPEC. OIA.
LUG DENSIIY $7194-4.4 \mathrm{MM}$
SPEC. LENGTH

\begin{tabular}{|c|c|c|c|c|c|c|}
\hline $\begin{array}{l}\text { SPFCIMEA } \\
\text { IUIMEER }\end{array}$ & \multicolumn{2}{|c|}{$\begin{array}{l}\text { ORIEATE } \\
\text { ATION }\end{array}$} & $\begin{array}{l}\text { LOCA- DENSITY } \\
\text { TION (MG/M: }\end{array}$ & $\begin{array}{l}\text { MODULUS OF } \\
\text { RUIPTURE (MPA) } \\
\text { (UNCORKECTED) }\end{array}$ & $\begin{array}{l}\text { FLEXURAL } \\
\text { STRENGTH } \\
\text { ICORRECTE }\end{array}$ & $\begin{array}{l}\text { L } \\
\text { (MPA) } \\
\text { ED) }\end{array}$ \\
\hline $3 A C$ & $\begin{array}{l}1 \cap 2 A \\
106 A \\
106 A \\
112 A \\
112 A \\
120 A \\
120 A \\
124 A \\
124 \mathrm{~A} \\
128 A \\
132 A \\
134 A \\
136 A \\
142 A \\
142 B \\
152 A \\
152 A \\
154 A \\
154 A \\
154 A\end{array}$ & $\begin{array}{l}\Delta x \\
\Delta x \\
\Delta x \\
\Delta x \\
\Delta x \\
\Delta x \\
\Delta x \\
\Delta x \\
\Delta x \\
\Delta x \\
\Delta x \\
\Delta x \\
\Delta x \\
\Delta x \\
\Delta x \\
\Delta x \\
\Delta x \\
\Delta x \\
\Delta x \\
\Delta x\end{array}$ & $\begin{array}{l}M L C \\
M L C \\
M L C \\
M L C \\
M L C \\
M L C \\
M L C \\
M L C \\
M L C \\
M L C \\
M L C \\
M L C \\
M L C \\
M L C \\
B L C \\
M L C \\
M L C \\
M L C \\
M L C \\
M L C\end{array}$ & $\begin{array}{l}24.4 \\
24.7 \\
19.5 \\
23.4 \\
26.2 \\
21.8 \\
25.1 \\
28.0 \\
21.7 \\
26.9 \\
22.6 \\
25.2 \\
26.7 \\
29.2 \\
23.6 \\
23.0 \\
32.6 \\
25.8 \\
29.9 \\
26.5\end{array}$ & $\begin{array}{l}21.3 \\
21.5 \\
17.6 \\
20.9 \\
22.6 \\
19.4 \\
21.8 \\
23.9 \\
19.3 \\
23.1 \\
20.0 \\
21.9 \\
22.9 \\
24.6 \\
21.8 \\
20.3 \\
26.7 \\
22.3 \\
25.1 \\
22.8\end{array}$ & \\
\hline & & ME AI & 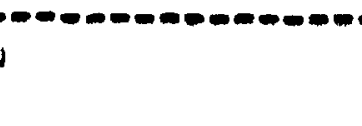 & $\begin{array}{l}25.4 \text { MPA } \\
(3678 . \text { PST) }\end{array}$ & $\begin{array}{r}22.0 \\
13194 .\end{array}$ & $\begin{array}{l}\text { MPA } \\
\text { PSII) }\end{array}$ \\
\hline & & STD & UEV. & 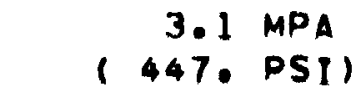 & 1314 & $\begin{array}{l}\text { MPA } \\
\text { PSII }\end{array}$ \\
\hline
\end{tabular}


TABLE B-4 (Continued)

FLEXUMAL PROPFPTIES OF H=45I GRAPHITE

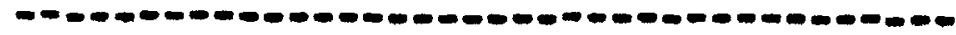

LOT NO.
LOC NPEC. DIA.

\begin{tabular}{|c|c|c|c|c|c|c|}
\hline $\begin{array}{l}\text { PEC I MEN } \\
\text { MIIMAEE }\end{array}$ & \multicolumn{2}{|c|}{$\begin{array}{l}\text { OHIFNT- } \\
\text { ATION }\end{array}$} & $\begin{array}{l}\text { LOCA- DENSITY } \\
\text { TION (MG/HOST) }\end{array}$ & $\begin{array}{l}\text { MODULUS OF } \\
\text { HUPTURE (MPA) } \\
\text { (UNCORRECTED) }\end{array}$ & $\begin{array}{l}\text { FLFXIIRAL } \\
\text { STRFNGTH } \\
\text { ICORRECTE }\end{array}$ & $\begin{array}{l}\text { L } \\
\text { (MPA) } \\
\text { ED) }\end{array}$ \\
\hline $3 \mathrm{PC}$ & $\begin{array}{l}161 \\
165 \\
167 \\
171 \\
173 \\
183 \\
185 \\
189 \\
191 \\
195 \\
201 \\
205 \\
207 \\
211 \\
213 \\
223 \\
225 \\
229 \\
231 \\
235\end{array}$ & 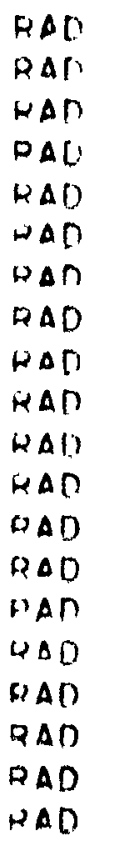 & $\begin{array}{l}M L C \\
M L C \\
M L C \\
M L C \\
M L C \\
M L C \\
M L C \\
M L C \\
M L C \\
M L C \\
M L C \\
M L C \\
M L C \\
M L C \\
M L C \\
M L C \\
M L C \\
M L C \\
M L C \\
M L C\end{array}$ & $\begin{array}{l}26.7 \\
19.0 \\
23.3 \\
21.9 \\
21.2 \\
17.7 \\
22.2 \\
22.4 \\
22.5 \\
21.4 \\
20.6 \\
20.0 \\
21.5 \\
25.6 \\
21.9 \\
25.4 \\
19.2 \\
27.2 \\
22.2 \\
21.9\end{array}$ & $\begin{array}{l}22.3 \\
16.9 \\
20.0 \\
19.1 \\
18.5 \\
15.9 \\
19.2 \\
19.7 \\
19.5 \\
18.7 \\
18.1 \\
17.6 \\
18.0 \\
21.5 \\
19.0 \\
21.4 \\
17.0 \\
22.6 \\
19.2 \\
19.1\end{array}$ & \\
\hline & & MEAR & & $\begin{array}{c}22.2 \text { MPA } \\
(3216 . \text { PSI })\end{array}$ & $\begin{array}{r}19.2 \\
12781\end{array}$ & $\begin{array}{l}\text { MPA } \\
\text { PSII }\end{array}$ \\
\hline & & STD. & DEV. & $\begin{array}{c}2.5 \text { MPA } \\
(367 . \text { PSI })\end{array}$ & $\begin{array}{r}1.9 \\
(256 .\end{array}$ & $\begin{array}{l}\text { MPA } \\
\text { PSI) }\end{array}$ \\
\hline
\end{tabular}


TABLE B-4 (Continued)

FIEAURAL PHORFRTIFS OF H-45I GRAPHITF

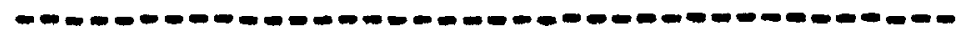
LIT No).
SPEC. DIA.
Leic, ori.
SPEC. LENGTH
$6.4 \mathrm{NM}$
LUE, DFNSIIY
$7194-40$ $19 / 11003$
0. $M M$

\begin{tabular}{|c|c|c|c|c|c|c|}
\hline $\begin{array}{l}\text { SOECIMEN } \\
\text { WIMAER }\end{array}$ & \multicolumn{2}{|c|}{$\begin{array}{c}\text { UPIFWTE } \\
\text { MTINII }\end{array}$} & $\begin{array}{l}\text { LUCA- NENSITY } \\
1101+(4 G / M \triangle 3)\end{array}$ & $\begin{array}{l}\text { MUDULISS OF } \\
\text { RIIP TURE (MP AA) } \\
\text { (IIPCISHRECTED) }\end{array}$ & $\begin{array}{l}\text { FLEXIIRAL } \\
\text { SIRENGTH } \\
\text { ICORRECTE }\end{array}$ & $\begin{array}{l}\text { L } \\
\text { EOPA) } \\
\text { EOPA) }\end{array}$ \\
\hline $3 \cap \mathrm{E}$ & 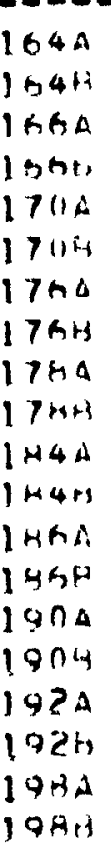 & $\begin{array}{l}\Delta x \\
\Delta x \\
\Delta x \\
\Delta x \\
\Delta x \\
\Delta x \\
\Delta x \\
\Delta x \\
\Delta x \\
\Delta x \\
A x \\
\Delta x \\
\Delta x \\
\Delta x \\
\Delta x \\
\Delta x \\
\Delta x \\
\Delta x \\
\Delta x \\
\Delta x\end{array}$ & 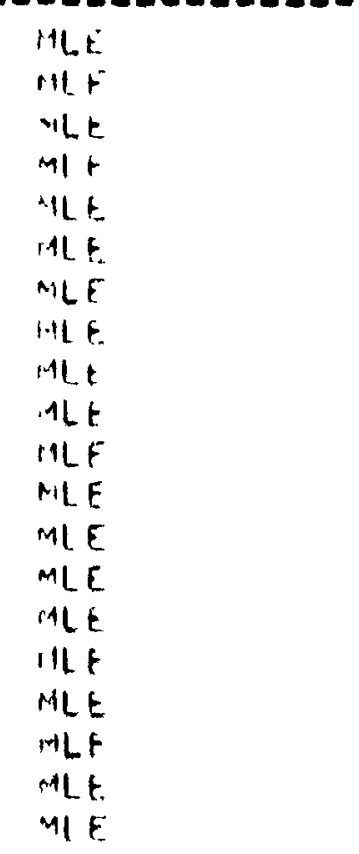 & $\begin{array}{l}33.6 \\
34.8 \\
31.1 \\
32.4 \\
33.9 \\
29.2 \\
32.9 \\
32.0 \\
30.3 \\
27.2 \\
35.7 \\
30.4 \\
31.5 \\
35.0 \\
31.1 \\
37.5 \\
35.2 \\
33.8 \\
34.1 \\
32.3\end{array}$ & $\begin{array}{l}28.1 \\
29.4 \\
26.4 \\
27.3 \\
24.3 \\
25.1 \\
27.4 \\
27.1 \\
25.9 \\
23.8 \\
29.4 \\
25.9 \\
26.7 \\
29.0 \\
25.8 \\
30.4 \\
29.1 \\
28.7 \\
28.4 \\
27.2\end{array}$ & \\
\hline & & $N \in A T$ & & $\begin{array}{l}37.7 \text { MPA } \\
(4737 \text {. PSI) }\end{array}$ & $\begin{array}{r}27.4 \\
13977 .\end{array}$ & $\begin{array}{l}\text { MPA } \\
\text { PSII }\end{array}$ \\
\hline & & STU. & UFV. & $\begin{array}{r}2.5: 4 P A \\
(362 . P S 1)\end{array}$ & $\begin{array}{r}1.6 \\
1235 .\end{array}$ & $\begin{array}{l}\text { MPA } \\
\text { PSII }\end{array}$ \\
\hline
\end{tabular}


TABLE B-4 (Continued)

FLE XIRAL PROPERTIFS OF H=45I GRAPHTTE

FLPXIIRAL DK

LOT IN. SPEC. DIA. $6.4 \mathrm{MM}$

LOG NO. $7194-49$ SPEC. LENGTH O. MM

LOM NENSITY MG/M\$?

\begin{tabular}{|c|c|c|c|c|c|c|}
\hline $\begin{array}{l}\text { FFCIAFN } \\
\text { PHINAER }\end{array}$ & \multicolumn{2}{|c|}{$\begin{array}{l}\text { ORIEVT- } \\
\text { ATTON }\end{array}$} & $\begin{array}{l}\text { LUCA- DENSITY } \\
\text { TION (M(B/WO3) }\end{array}$ & $\begin{array}{l}\text { HODULUS OF } \\
\text { HUPTIIRE (WPA) } \\
\text { (INCORRECTED) }\end{array}$ & $\begin{array}{l}\text { FLEXIIRA } \\
\text { STRENGTH } \\
\text { ICORRECT }\end{array}$ & $\begin{array}{l}L \\
(M P A) \\
(D)\end{array}$ \\
\hline उAE & $\begin{array}{l}241 \\
245 \\
247 \\
251 \\
253 \\
203 \\
207 \\
249 \\
273 \\
275 \\
281 \\
285 \\
247 \\
291 \\
293 \\
303 \\
305 \\
309 \\
311 \\
315\end{array}$ & 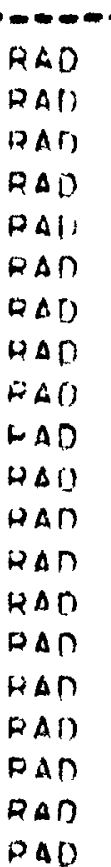 & $\begin{array}{l}\text { MLE } \\
\text { MLE } \\
M L E \\
M I E \\
\text { MLE } \\
\text { MILE } \\
\text { MLE } \\
\text { MLE } \\
\text { MLE } \\
\text { MLE } \\
\text { MLE } \\
\text { MLE } \\
\text { MLE } \\
\text { MLE } \\
M L E \\
\text { MLE } \\
\text { MLE } \\
\text { MLEE } \\
\text { MLE } \\
\text { MLLE }\end{array}$ & $\begin{array}{l}22.8 \\
30.9 \\
28.1 \\
26.9 \\
26.8 \\
22.3 \\
25.3 \\
21.5 \\
24.4 \\
23.3 \\
28.6 \\
28.0 \\
29.3 \\
25.6 \\
30.2 \\
23.4 \\
28.7 \\
27.1 \\
23.9 \\
28.4\end{array}$ & $\begin{array}{l}19.9 \\
25 . ? \\
23.5 \\
22.7 \\
22.6 \\
19.5 \\
21.6 \\
18.9 \\
21.0 \\
20.2 \\
23.9 \\
22.4 \\
24.3 \\
21.8 \\
24.8 \\
20.4 \\
23.9 \\
22.8 \\
20.7 \\
23.7\end{array}$ & \\
\hline & & MEA & & $\begin{array}{c}26.3 \text { MPA } \\
(3813 . P 5 I)\end{array}$ & $\begin{array}{r}22.2 \\
13226 .\end{array}$ & $\begin{array}{l}\text { MPA } \\
\text { PSII) }\end{array}$ \\
\hline & & STO & UEV. & $\begin{array}{c}2.8 \text { MPA } \\
(407 . \text { PST) }\end{array}$ & $\begin{aligned} 1.9 \\
1270 .\end{aligned}$ & $\begin{array}{l}\text { MPA } \\
\text { PSI) }\end{array}$ \\
\hline
\end{tabular}


TABLE B-5

FLEXURAL PROPERTIES OF H-451 GRAPHITF

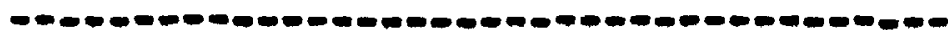

LOT NO.

LOG NO.

SPEC. OIA.

$6.4 \mathrm{MM}$

LOG DENSITY

$7194-52$

SPEC. LENGTH

$0 . M M$ $M G / M \cdot 3$

\begin{tabular}{|c|c|c|c|c|c|c|}
\hline $\begin{array}{l}\text { SPECIMFN } \\
\text { NUMAEED }\end{array}$ & \multicolumn{2}{|c|}{$\begin{array}{l}\text { DRIENT- } \\
\text { ATION }\end{array}$} & $\begin{array}{l}\text { LOCA- NENSITY } \\
\text { IION (MG/MOB) }\end{array}$ & $\begin{array}{l}\text { MODULUS OF } \\
\text { RUPTURE (MPA) } \\
\text { (UNCORRECTED) }\end{array}$ & $\begin{array}{l}\text { FLEXURA } \\
\text { STRENGTH } \\
\text { ICORRECT }\end{array}$ & $\begin{array}{l}\operatorname{l} \\
(M P A)\end{array}$ \\
\hline $1 A C$ & $\begin{array}{l}2 A \\
4 A \\
4 A \\
14 A \\
14 A \\
20 A \\
20 B \\
26 A \\
26 B \\
24 A \\
32 A \\
36 A \\
36 A \\
42 A \\
42 B \\
52 A \\
52 B \\
54 A \\
54 B \\
5 R A\end{array}$ & $\begin{array}{l}\Delta x \\
\Delta x \\
\Delta x \\
\Delta x \\
\Delta x \\
A x \\
\Delta x \\
A X \\
\Delta x \\
\Delta x \\
\Delta x \\
\Delta x \\
\Delta x \\
A x \\
\Delta x \\
A x \\
\Delta x \\
\Delta x \\
\Delta x \\
\Delta x\end{array}$ & $\begin{array}{l}E C \\
E C \\
E C \\
E C \\
E C \\
E C \\
E C \\
E C \\
E C \\
E C \\
E C \\
E C \\
E C \\
E C \\
E C \\
E C \\
E C \\
E C \\
E C \\
E C\end{array}$ & $\begin{array}{l}25.9 \\
27.5 \\
28.0 \\
16.0 \\
25.4 \\
20.1 \\
25.4 \\
24.7 \\
28.2 \\
30.4 \\
26.4 \\
28.8 \\
19.1 \\
24.8 \\
29.1 \\
29.4 \\
27.1 \\
23.2 \\
23.5 \\
29.6\end{array}$ & $\begin{array}{l}22.4 \\
23.6 \\
23.9 \\
14.8 \\
22.1 \\
18.1 \\
22.1 \\
21.6 \\
24.1 \\
25.5 \\
22.8 \\
24.4 \\
17.3 \\
21.6 \\
24.6 \\
24.8 \\
23.3 \\
20.4 \\
20.7 \\
24.9\end{array}$ & \\
\hline & & & & $\begin{array}{c}25.6 \text { MPA } \\
(3718 . \text { PSI) }\end{array}$ & $\begin{array}{r}22.2 \\
13213 .\end{array}$ & $\begin{array}{l}\text { MPA } \\
\text { PSII }\end{array}$ \\
\hline & & STO. & DEV. & $\begin{array}{c}3.8 \text { MPA } \\
(548 . \text { PSI) }\end{array}$ & $\begin{array}{r}2.8 \\
1404 .\end{array}$ & $\begin{array}{l}\text { MPA } \\
\text { PSII }\end{array}$ \\
\hline
\end{tabular}


TABLE B-5 (Continued)

FLF XIITAL PROPERTIES OF H=451 GRAPHITE

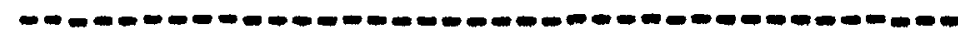

\begin{tabular}{|c|c|c|c|c|c|}
\hline $\begin{array}{l}\text { LOT } \\
\text { LOG }\end{array}$ & $\begin{array}{l}\text { NO: } \\
\text { No: }\end{array}$ & $7194-52$ & $\begin{array}{l}\text { SPEC. } \\
\text { SPEC. }\end{array}$ & $\begin{array}{l}\text { DIA. } \\
\text { LENGTH }\end{array}$ & $\begin{array}{r}0.4 \mathrm{MM} \\
0 . \mathrm{MM}\end{array}$ \\
\hline or: & DENSITY & & & & \\
\hline
\end{tabular}

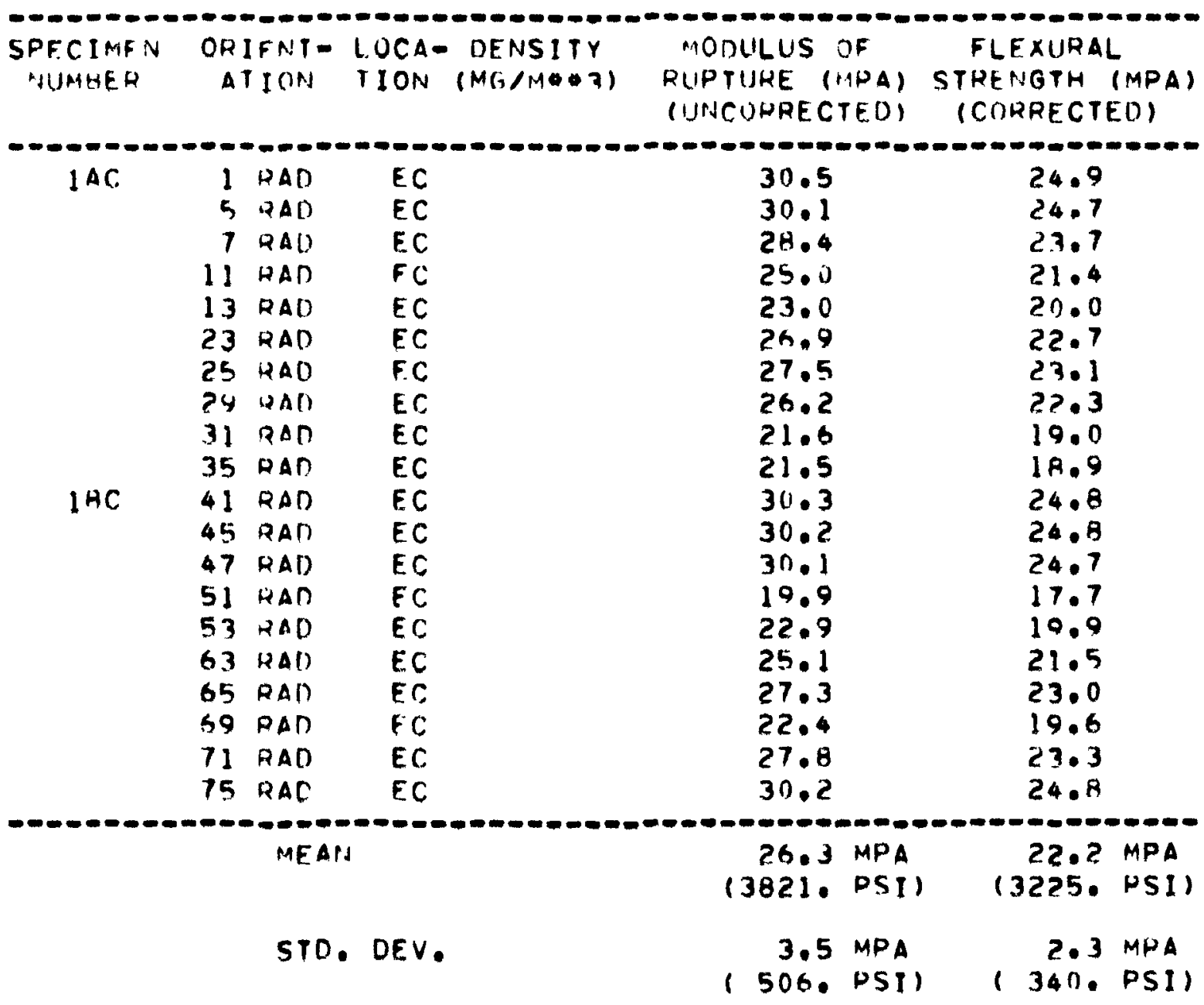


TABLE B-5 (Continued)

FLEXIIRAL PROPERTIES OF HO45I GRAPHITF
LOT NO.
LOG NO. SPEC. DIA.
LOG NENSITY $7194-5$ ?
LOG/M

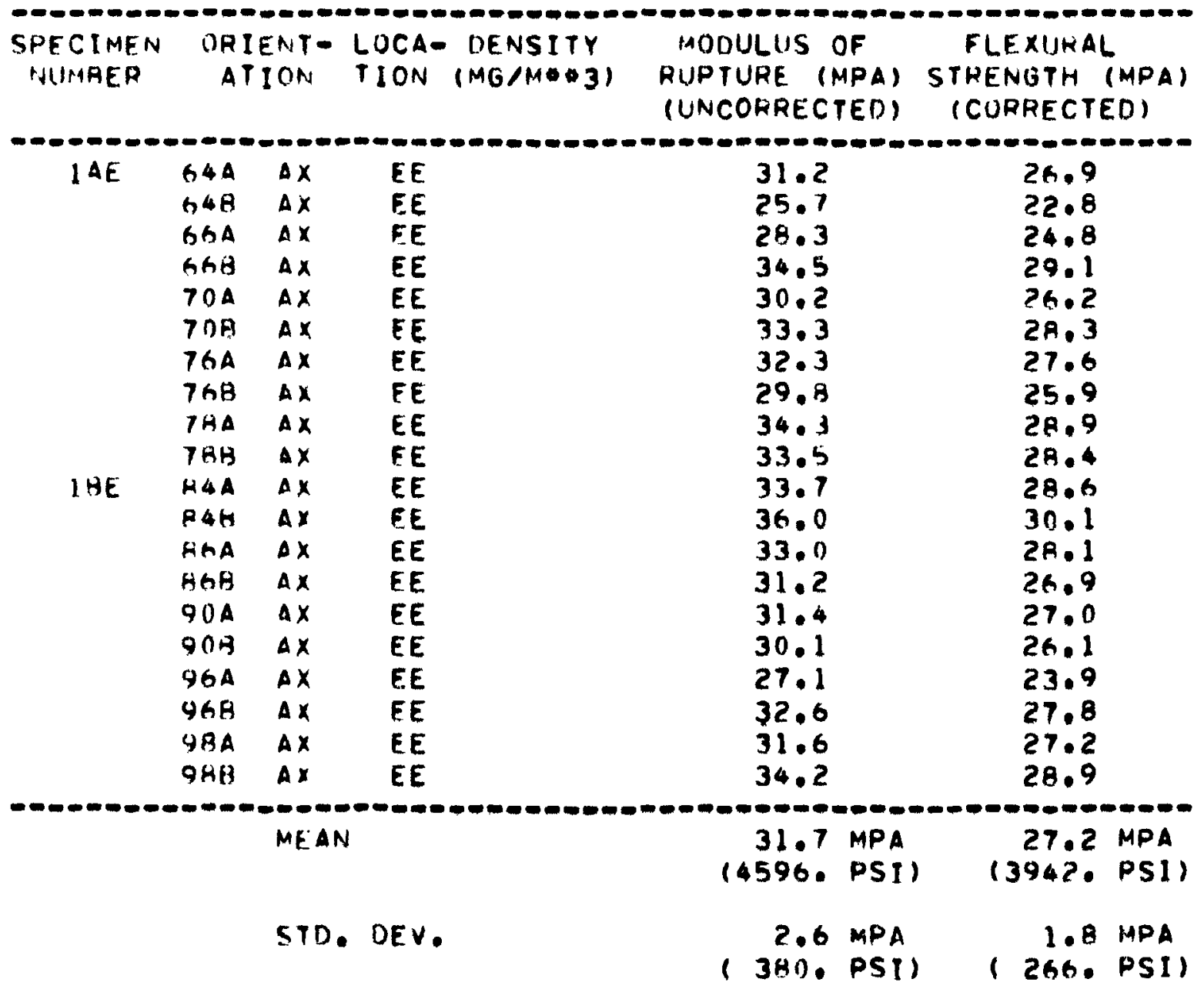


TABLE B-5 (Continued)

FLEXIRAL PROPERTIES OF M-451 GRAPHITE

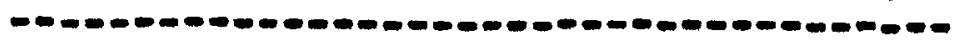

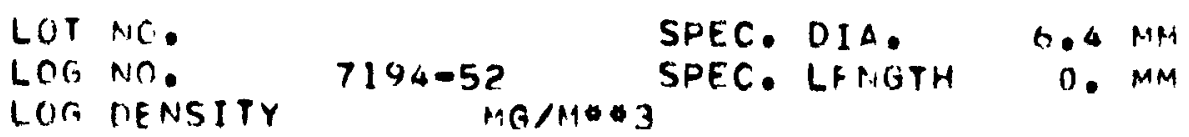

\begin{tabular}{|c|c|c|c|c|c|c|}
\hline $\begin{array}{l}\text { SPECIMEN" } \\
\text { NIIMAER }\end{array}$ & \multicolumn{2}{|c|}{$\begin{array}{l}\text { ORIENT- } \\
\text { ATIOI: }\end{array}$} & $\begin{array}{l}\text { LOCA- DENSITY } \\
\text { TION (MG/MOI) }\end{array}$ & $\begin{array}{l}\text { MODULUS OF } \\
\text { PUPTURE (MPA) } \\
\text { (INNCORRECTED) }\end{array}$ & $\begin{array}{l}\text { FLEXIIRAL } \\
\text { STRENGTH } \\
\text { ICORRECTE }\end{array}$ & $\begin{array}{l}L \\
\text { (MPA) } \\
\text { EO) }\end{array}$ \\
\hline $1 \Delta E$ & $\begin{array}{l}81 \\
85 \\
87 \\
91 \\
93 \\
103 \\
105 \\
109 \\
111 \\
115 \\
121 \\
125 \\
127 \\
131 \\
133 \\
143 \\
145 \\
149 \\
151 \\
155\end{array}$ & 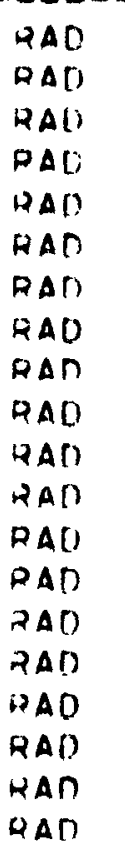 & $\begin{array}{l}E E \\
F E \\
E E \\
F E \\
E E \\
E E \\
E E \\
E E \\
E E \\
E E \\
E E \\
E E \\
E E \\
E E \\
E E \\
E E \\
E E \\
E E \\
E E \\
E E\end{array}$ & $\begin{array}{l}32.0 \\
28.5 \\
32.1 \\
29.5 \\
28.9 \\
26.5 \\
30.8 \\
30.4 \\
31.2 \\
29.8 \\
34.7 \\
32.6 \\
23.1 \\
28.9 \\
26.0 \\
28.8 \\
32.2 \\
32.1 \\
30.1 \\
30.8\end{array}$ & $\begin{array}{l}26.4 \\
24.2 \\
26.5 \\
24.8 \\
24.4 \\
22.8 \\
25.6 \\
25.4 \\
25.9 \\
25.0 \\
26.7 \\
26.8 \\
20.4 \\
24.4 \\
22.5 \\
24.4 \\
26.5 \\
26.5 \\
25.2 \\
25.7\end{array}$ & \\
\hline & & MEA $A$ & & $\begin{array}{c}29.9 \text { MPA } \\
(4343 . \text { PSI })\end{array}$ & $\begin{array}{r}25.1 \\
13536 .\end{array}$ & $\begin{array}{l}\text { MPA } \\
\text { PSII }\end{array}$ \\
\hline & & STD. & UEV. & $\begin{array}{c}2.6 \text { MPA } \\
(381 . \text { PSI }\end{array}$ & $\begin{array}{r}1.7 \\
1250 .\end{array}$ & $\begin{array}{l}\text { MPA } \\
\text { PSII }\end{array}$ \\
\hline
\end{tabular}


TABLE B-5 (Continued)

FLEYIRAL PKOPEFTIFS IF H=451 GRAPHTTE

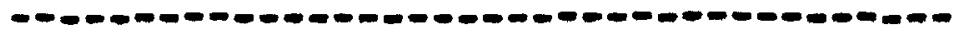

LUT NO.
LUG NOEC. DIA.
LUG NENSITY $7194-92$
SPFC. LFNGTH

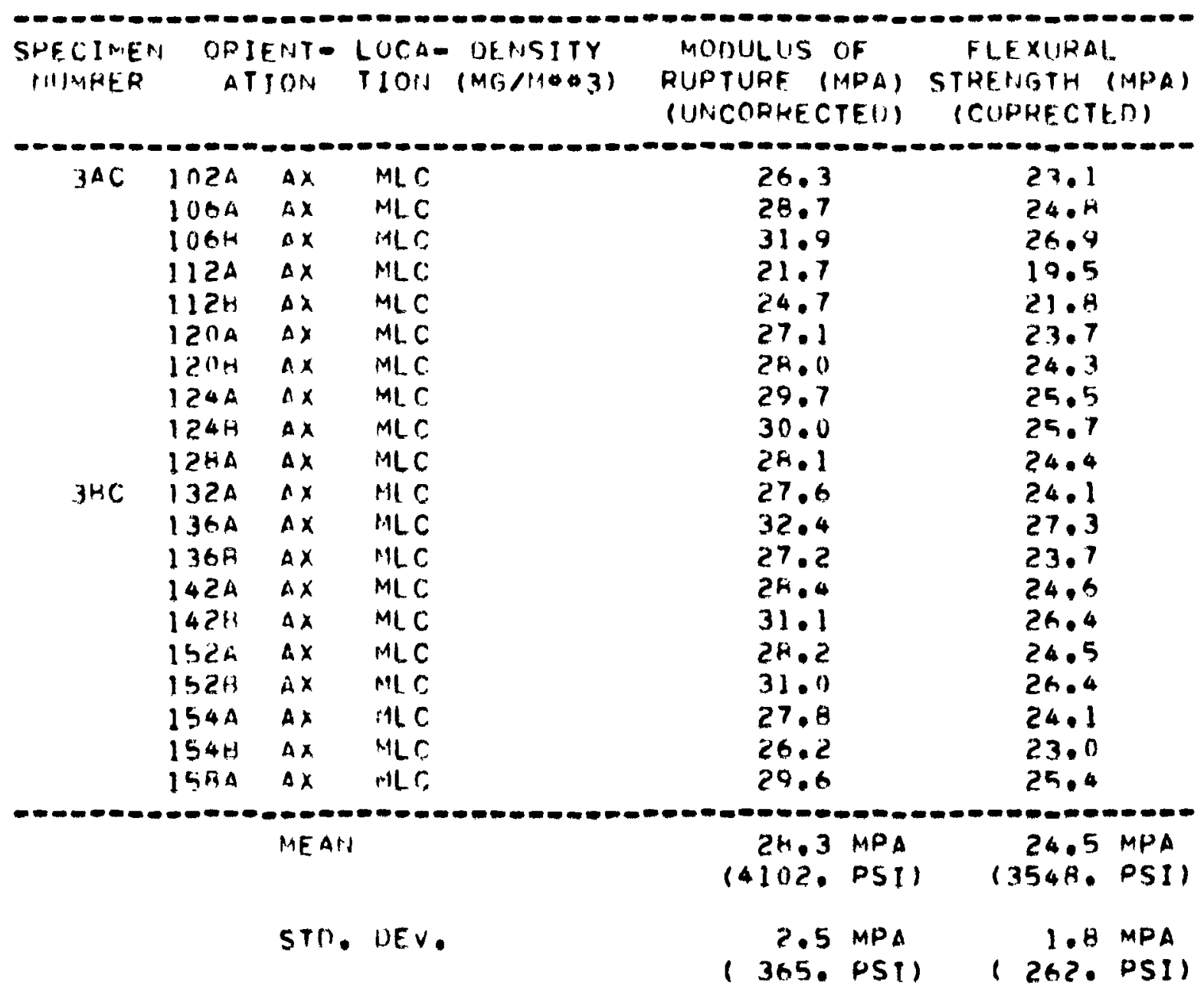


TABLE B-5 (Continued)

FLFXIJKAL PHOFEFTIFS (IF HO4SI GRAPHITE

LOT NO.
LOG NOE SPEC. DIA.
LOG MENSITY S.4 MM
LPEC. LENGTH

\begin{tabular}{|c|c|c|c|c|c|c|c|}
\hline $\begin{array}{l}\text { HEC CIMFN } \\
\text { PHIPEER }\end{array}$ & \multicolumn{2}{|c|}{$\begin{array}{l}\text { OHIEAT- } \\
\text { ATION }\end{array}$} & $\begin{array}{l}\text { LOCA = DENSITY } \\
\text { TIOH (M(G/MO3) }\end{array}$ & $\begin{array}{l}\text { NODULUS } \\
\text { RUPTURE I } \\
\text { IUNCUKREC }\end{array}$ & $\begin{array}{l}\text { OF } \\
(M P A) \\
\text { CTE[D) }\end{array}$ & $\begin{array}{l}\text { FLEXIIRAI } \\
\text { STRENGTH } \\
\text { ICORPECT }\end{array}$ & $\begin{array}{l}\text { L } \\
\text { (MPA) } \\
E(D)\end{array}$ \\
\hline $3 \wedge \mathrm{C}$ & $\begin{array}{l}161 \\
165 \\
167 \\
171 \\
173 \\
173 \\
185 \\
189 \\
191 \\
195 \\
201 \\
205 \\
207 \\
211 \\
213 \\
223 \\
225 \\
229 \\
231 \\
235\end{array}$ & 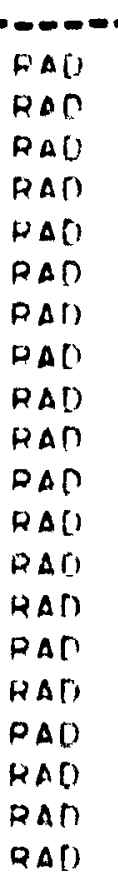 & $\begin{array}{l}\text { ALC } \\
M L C \\
M L C \\
M L C \\
M L C \\
M L C \\
M L C \\
M L C \\
M L C \\
M L C \\
M L C \\
M L C \\
M L C \\
M L C \\
M L C \\
M L C \\
M L C \\
M L C \\
M L C \\
M L C\end{array}$ & $\begin{array}{r}20.1 \\
2.3 .3 \\
22.5 \\
21.5 \\
22.5 \\
17.4 \\
11.9 \\
21.2 \\
9.0 \\
20.1 \\
16.8 \\
21.9 \\
20.7 \\
20.2 \\
20.1 \\
22.8 \\
22.5 \\
24.5 \\
26.8 \\
28.4\end{array}$ & $\begin{array}{l}0 \\
1 \\
3 \\
5 \\
5 \\
5 \\
4 \\
7 \\
2 \\
0 \\
1 \\
3 \\
9 \\
7 \\
2 \\
1 \\
3 \\
5 \\
5 \\
3 \\
4\end{array}$ & $\begin{array}{l}17.9 \\
20.1 \\
19.6 \\
19.4 \\
19.5 \\
15.6 \\
11.2 \\
18.6 \\
19.5 \\
17.7 \\
15.2 \\
19.1 \\
18.2 \\
17.8 \\
17.8 \\
19.8 \\
19.6 \\
20.4 \\
22.4 \\
23.4\end{array}$ & \\
\hline & & MEAR & & $\begin{array}{r}20.7 \\
13003 .\end{array}$ & $\begin{array}{l}\text { MPA } \\
\text { PSII }\end{array}$ & $\begin{array}{r}14.1 \\
12621 .\end{array}$ & $\begin{array}{l}\text { MPA } \\
\text { PSII }\end{array}$ \\
\hline & & STD & DE $V$. & $\begin{array}{r}4.4 \\
1645 .\end{array}$ & $\begin{array}{l}\text { MPA } \\
\text { PSII }\end{array}$ & $\begin{array}{r}3.4 \\
1498\end{array}$ & $\begin{array}{l}\text { MPA } \\
\text { PSII }\end{array}$ \\
\hline
\end{tabular}


TABLE B-5 (Continued)

\section{FLEXURAL PROPERTIES OF H=451 GRAPHITE}

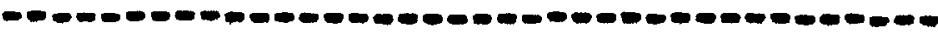

\begin{tabular}{|c|c|c|c|c|c|}
\hline $\begin{array}{l}10 T \\
\text { LOG }\end{array}$ & $\begin{array}{l}\text { NO. } \\
\text { NO. }\end{array}$ & $7194-52$ & $\begin{array}{l}\text { SPEC } \\
\text { SPEC }\end{array}$ & $\begin{array}{l}\text { DIA. } \\
\text { LENGTH }\end{array}$ & $\begin{array}{r}6.4 \mathrm{MM} \\
0 . \mathrm{MM}\end{array}$ \\
\hline 0 & DENSITY & & & & \\
\hline
\end{tabular}

SFECIMEN ORIENT- LUCA- OENSITY
NIMHER MOOULUS OF $\begin{gathered}\text { FLEXIRAL } \\ \text { RUPTURE (MPA) STRENGTH (MPA) } \\ \text { (UNCORRECTED) } \\ \text { (CORRECTEDI }\end{gathered}$


TABLE B-5 (Continued)

FLEXIRAL PRUPERTIES OF H-45I GRAPHTTE

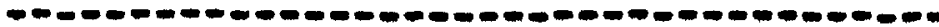

\begin{tabular}{|c|c|c|c|c|c|}
\hline LOT & $\begin{array}{l}\text { NO. } \\
\text { NG. }\end{array}$ & $7194-52$ & $\begin{array}{l}\text { SPEC. } \\
\text { SPEC. }\end{array}$ & $\begin{array}{l}\text { DIA. } \\
\text { LENGTH }\end{array}$ & $\begin{array}{r}6.4 \mathrm{MM} \\
0 . \mathrm{MM}\end{array}$ \\
\hline & DENSITY & & & & \\
\hline
\end{tabular}

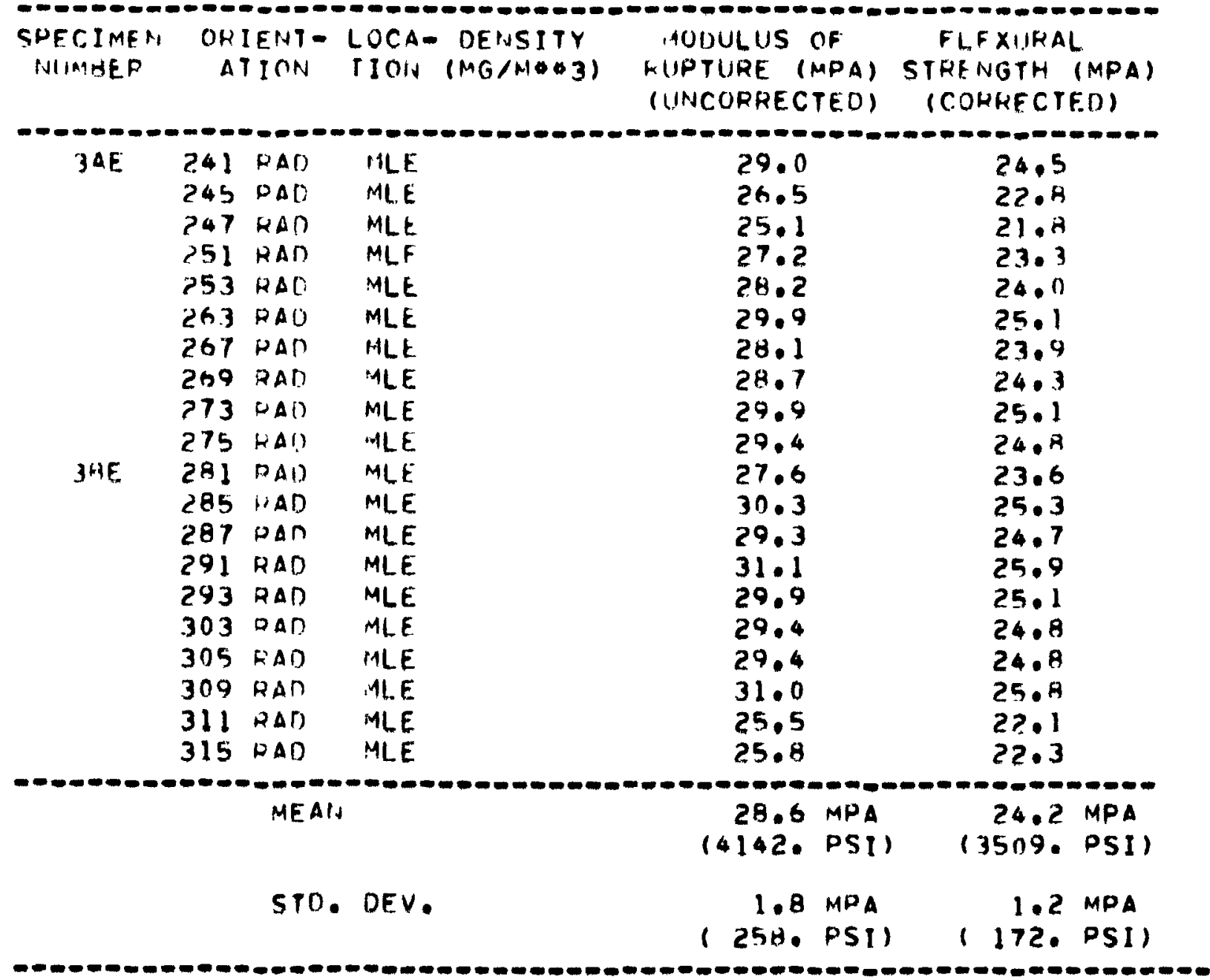


TABLE B-6

FLEXURAL PROPERTIES RF H=45I GRAPHITF

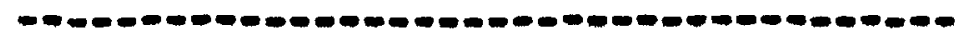

$\begin{array}{llll}\text { LOT INO. } & \text { SPEC. DIA. } & 6.4 \mathrm{MM} \\ \text { LOG NOE. } & 7194-87 & \text { SPEC. LENGTH } & 51 . \mathrm{MM} \\ \text { LOG NENSITY } & \text { MG/M } 3 & \end{array}$

\begin{tabular}{|c|c|c|c|c|c|c|}
\hline $\begin{array}{l}\text { SDECIMFN } \\
\text { NIIMAER }\end{array}$ & \multicolumn{2}{|c|}{$\begin{array}{l}\text { JRIENT- } \\
\text { ATION }\end{array}$} & $\begin{array}{l}\text { LOCA DENSITY } \\
\text { IION }(M G / M * 3)\end{array}$ & $\begin{array}{l}\text { MODULUS OF } \\
\text { RUPTURE (MPA) } \\
\text { (UNCORRECTED) }\end{array}$ & $\begin{array}{l}\text { FLEXIIRAI } \\
\text { STRENGTH } \\
\text { ICORRECTI }\end{array}$ & $\begin{array}{l}\text { L } \\
\text { (MPA) } \\
\text { ED) }\end{array}$ \\
\hline$I A C$ & $\begin{array}{l}7 A \\
4 A \\
4 A \\
14 A \\
14 B \\
20 A \\
20 A \\
26 A \\
26 A \\
28 A \\
32 A \\
36 A \\
36 A \\
42 A \\
42 B \\
52 A \\
52 A \\
54 A \\
54 B \\
58 A\end{array}$ & 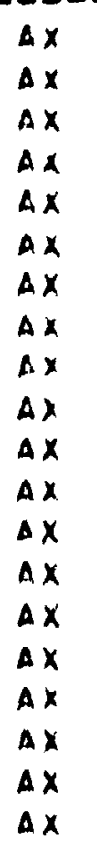 & $\begin{array}{l}E C \\
E C \\
E C \\
E C \\
E C \\
E C \\
E C \\
E C \\
E C \\
E C \\
E C \\
E C \\
E C \\
E C \\
E C \\
E C \\
E C \\
E C \\
E C \\
F C\end{array}$ & $\begin{array}{l}27.4 \\
32.5 \\
33.2 \\
31.4 \\
28.0 \\
28.1 \\
26.4 \\
26.4 \\
25.2 \\
27.9 \\
27.0 \\
27.7 \\
18.0 \\
32.0 \\
28.3 \\
29.3 \\
28.4 \\
30.2 \\
32.4 \\
27.1\end{array}$ & $\begin{array}{l}23.6 \\
27.0 \\
27.4 \\
26.6 \\
24.0 \\
24.1 \\
22.9 \\
23.1 \\
27.0 \\
24.0 \\
23.4 \\
23.9 \\
16.4 \\
26.7 \\
24.3 \\
24.9 \\
24.3 \\
25.5 \\
26.9 \\
23.4\end{array}$ & \\
\hline & & MEAI & & $\begin{array}{l}28.4 \text { MPA } \\
(4117 . \text { PSt) }\end{array}$ & $\begin{array}{r}24.2 \\
1351.4 .\end{array}$ & $\begin{array}{l}\text { MPA } \\
\text { PSII }\end{array}$ \\
\hline & & STD & DEV. & $\begin{array}{l}3.4 M P A \\
(492 . P S I)\end{array}$ & 1349. & $\begin{array}{l}\text { MPA } \\
\text { PSII }\end{array}$ \\
\hline
\end{tabular}


TABLE B-6 (Continued)

FLEXIIRAL PROPERTIES OF H=45I GRAPHITE

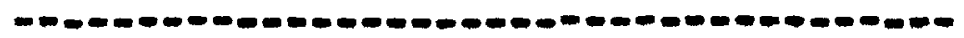

\begin{tabular}{|c|c|c|c|c|c|}
\hline & $\begin{array}{l}\text { N.1O. } \\
\text { NO. }\end{array}$ & $7194=87$ & $\begin{array}{l}\text { SPEC. } \\
\text { SPEC. }\end{array}$ & $\begin{array}{l}\text { OIA. } \\
\text { LENGTH }\end{array}$ & $\begin{array}{l}6.4 \mathrm{MM} \\
51 . \mathrm{MM}\end{array}$ \\
\hline 0 & DENSITY & & & & \\
\hline
\end{tabular}

\begin{tabular}{|c|c|c|c|c|}
\hline $\begin{array}{l}\text { SFECINES } \\
\text { NIIMAEK }\end{array}$ & $\begin{array}{l}\text { ORIEHT- } \\
\text { ATION }\end{array}$ & $\begin{array}{l}\text { IUCA- DENSITY } \\
\text { TION (MG/MOOZ) }\end{array}$ & $\begin{array}{l}\text { MODULUS OF } \\
\text { RUPTURE (MPA) } \\
\text { (INCOORECTED) }\end{array}$ & $\begin{array}{l}\text { FLEXUHAL } \\
\text { STRENGTH (MPA) } \\
\text { (CURRECTED) }\end{array}$ \\
\hline
\end{tabular}

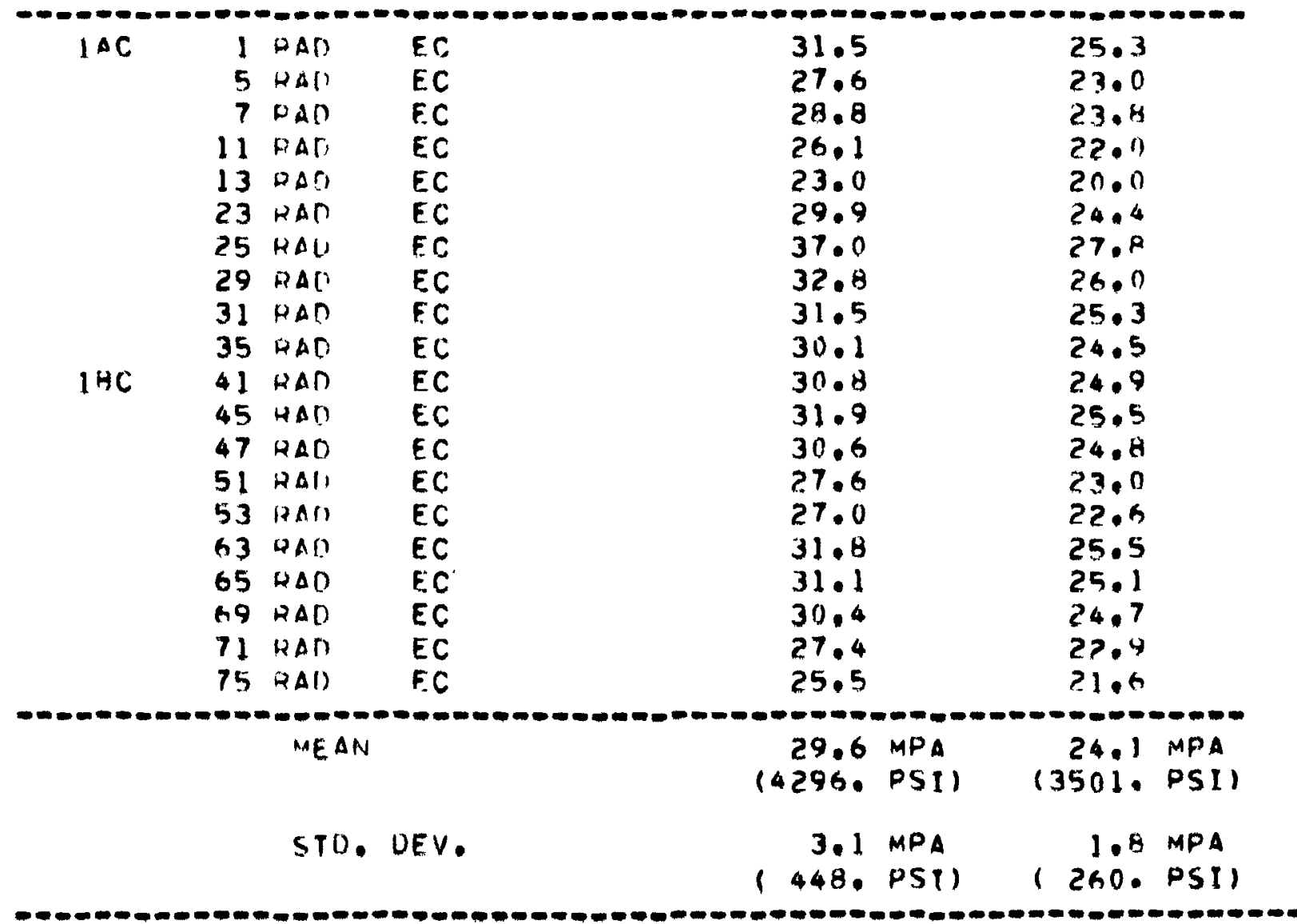


TABLE B-6 (Continued)

'LLEUPAL PPOPEPTIES OF H-45I GRAPHITE

-
LOT INO.
SPEC. LIA.
$6.4 M M$
LOE iNO.
$7194-87$
SPEC. LENGTH
:1. $M M$
LOG DENSITY $M C / M * 3$

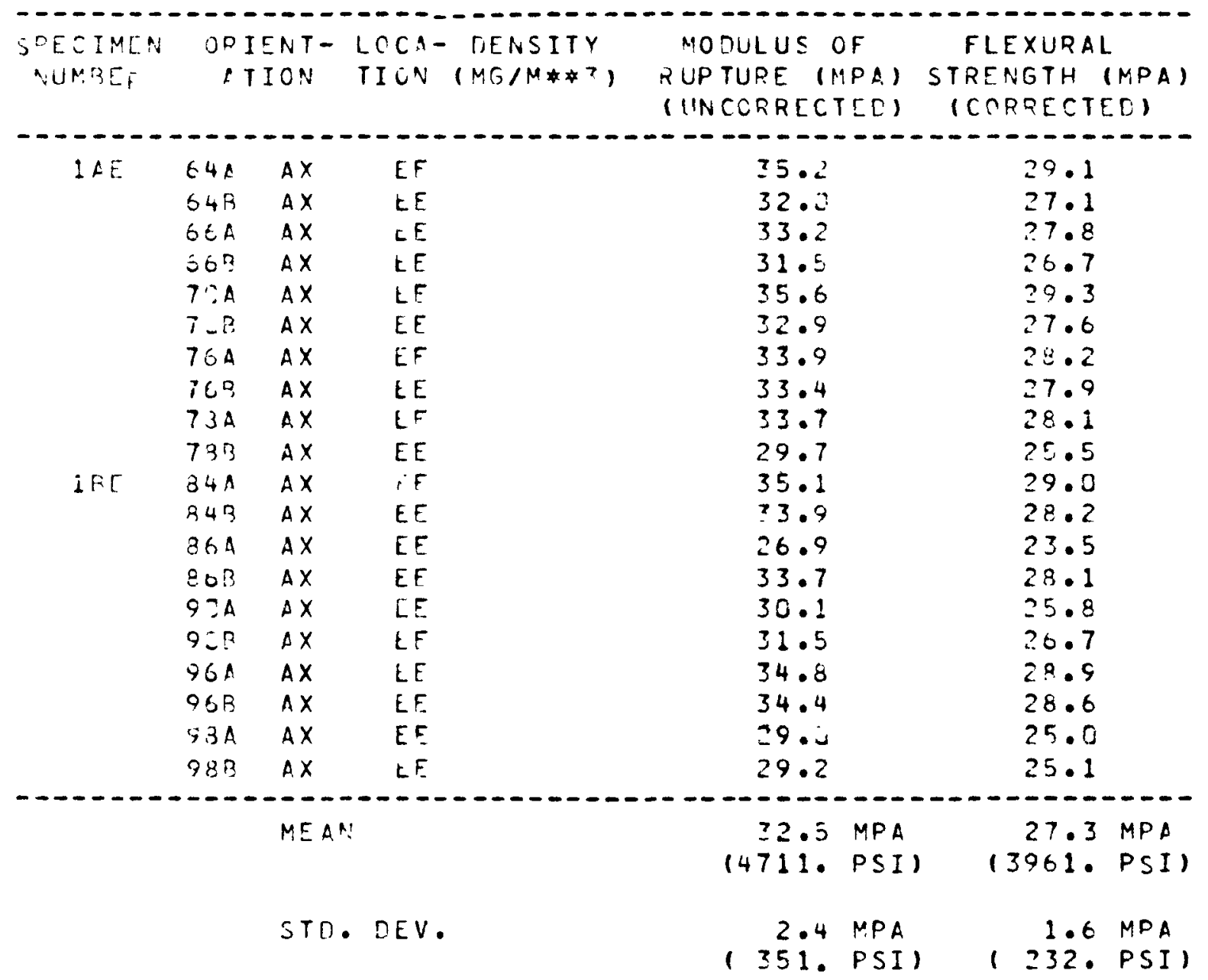


TABLE B-6 (Continued)

TLEXUFAL FROPERTIES OF H-451 GRAPHITE

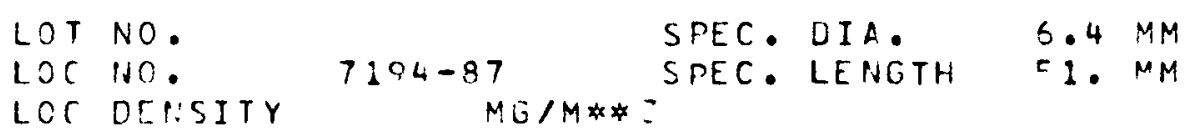

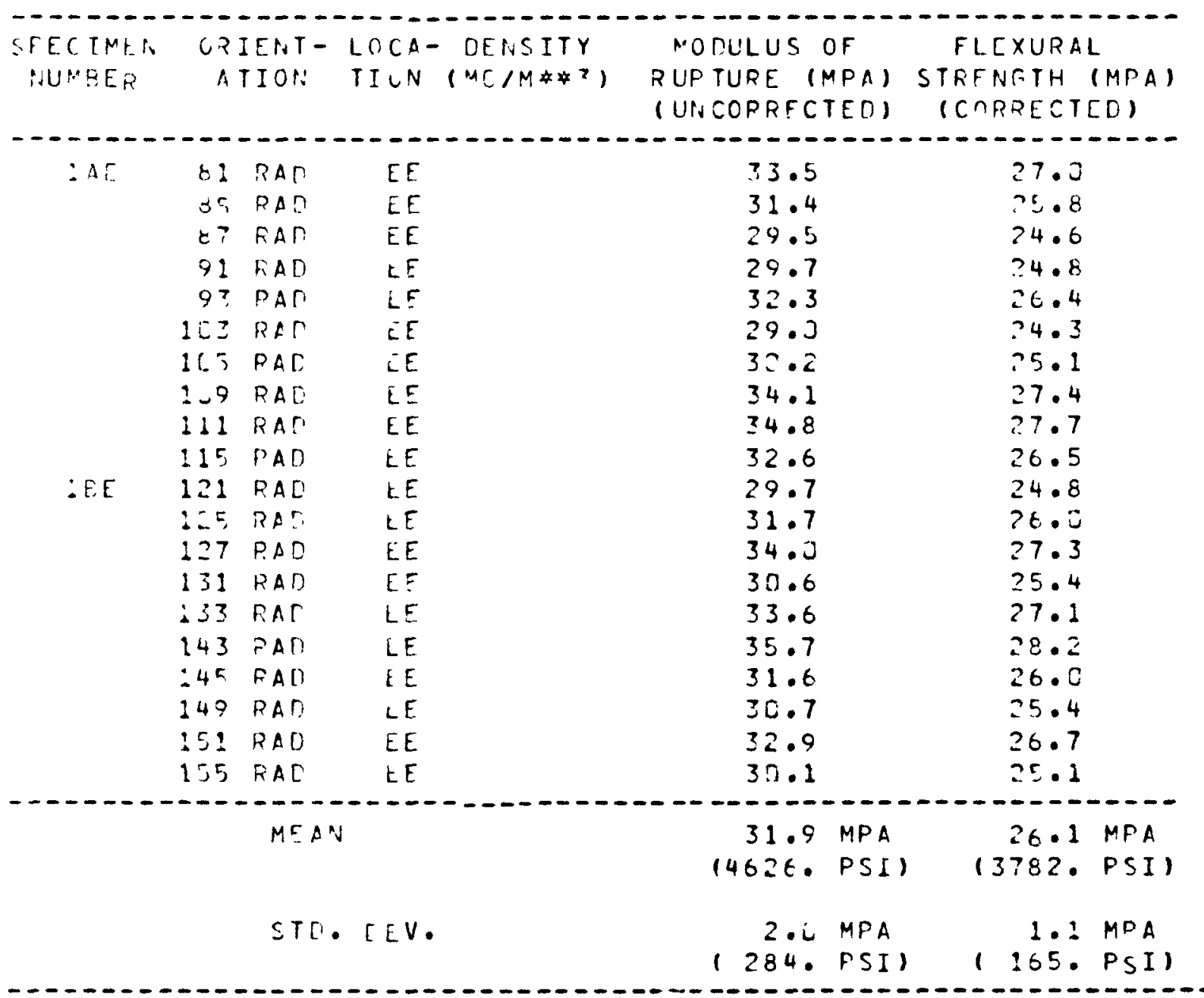


TABLE B-6 (Continued)

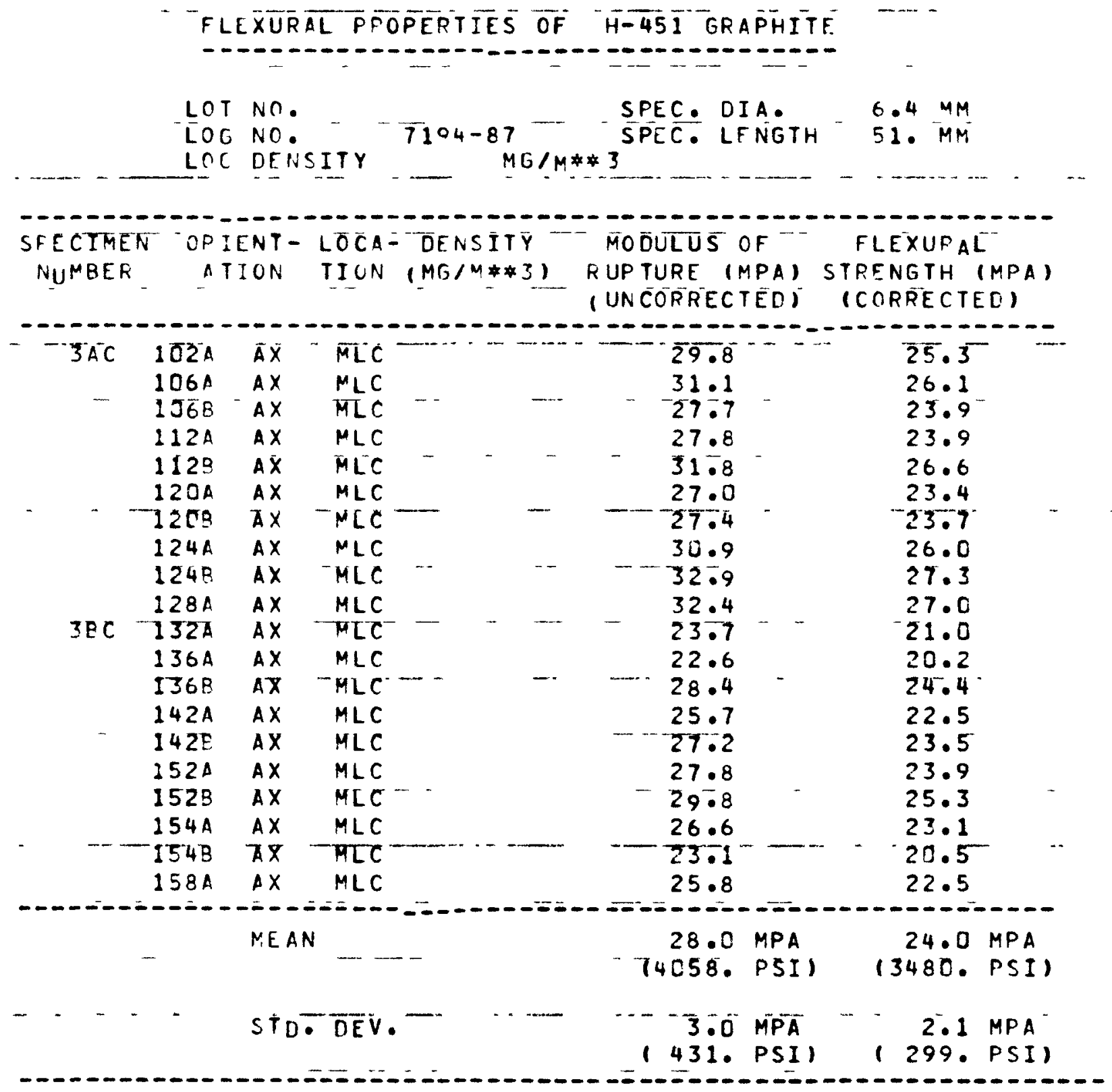


TABLE B-6 (Continued)

FLEXURAL PROPERTIES OF H-45! GR̈APHITE

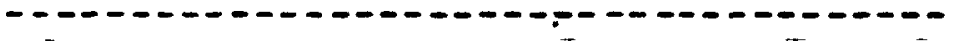

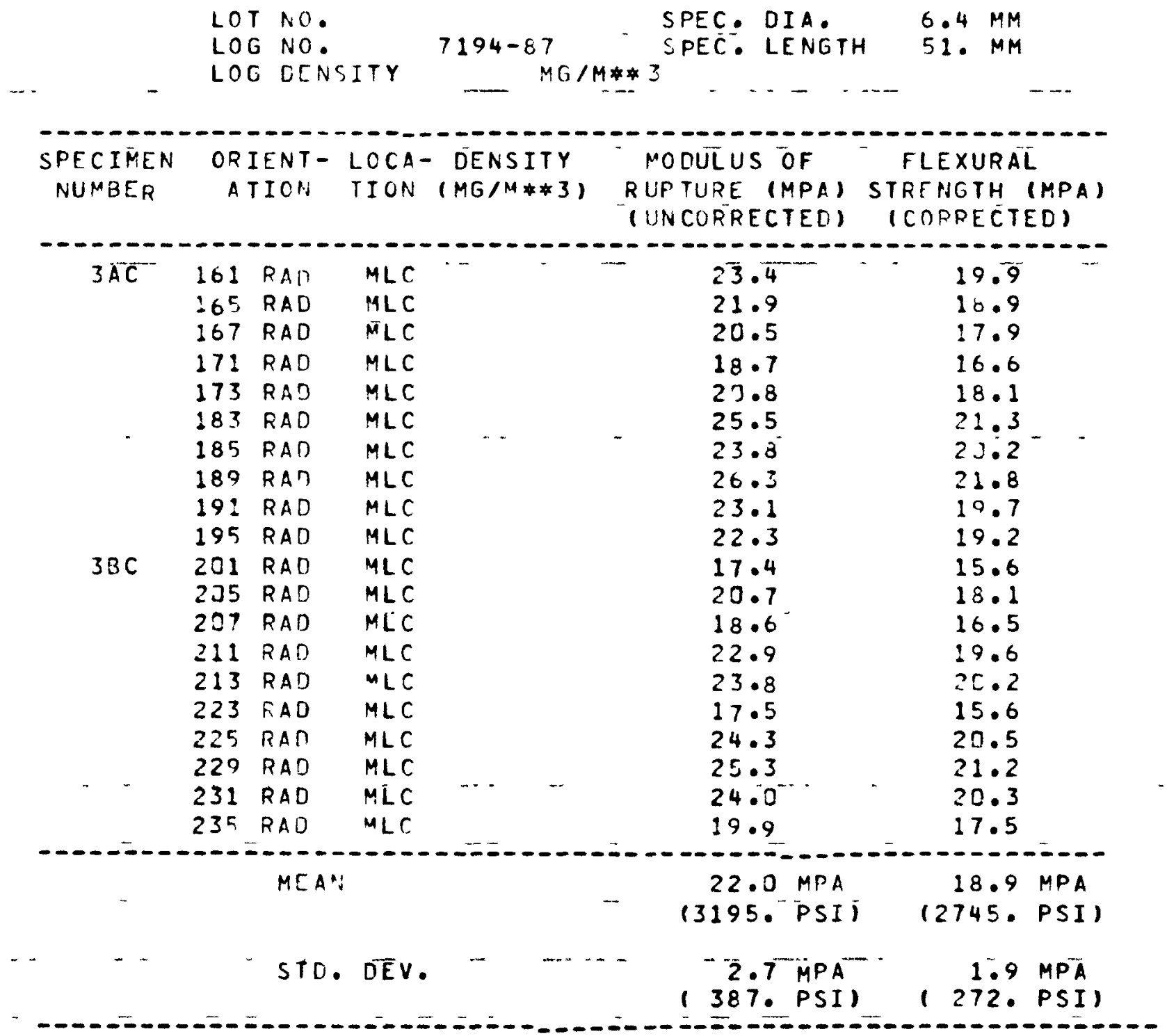


TABLE B-6 (Continued)

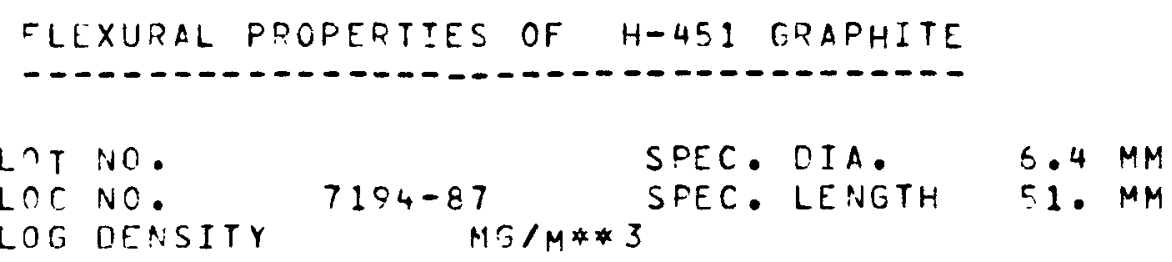

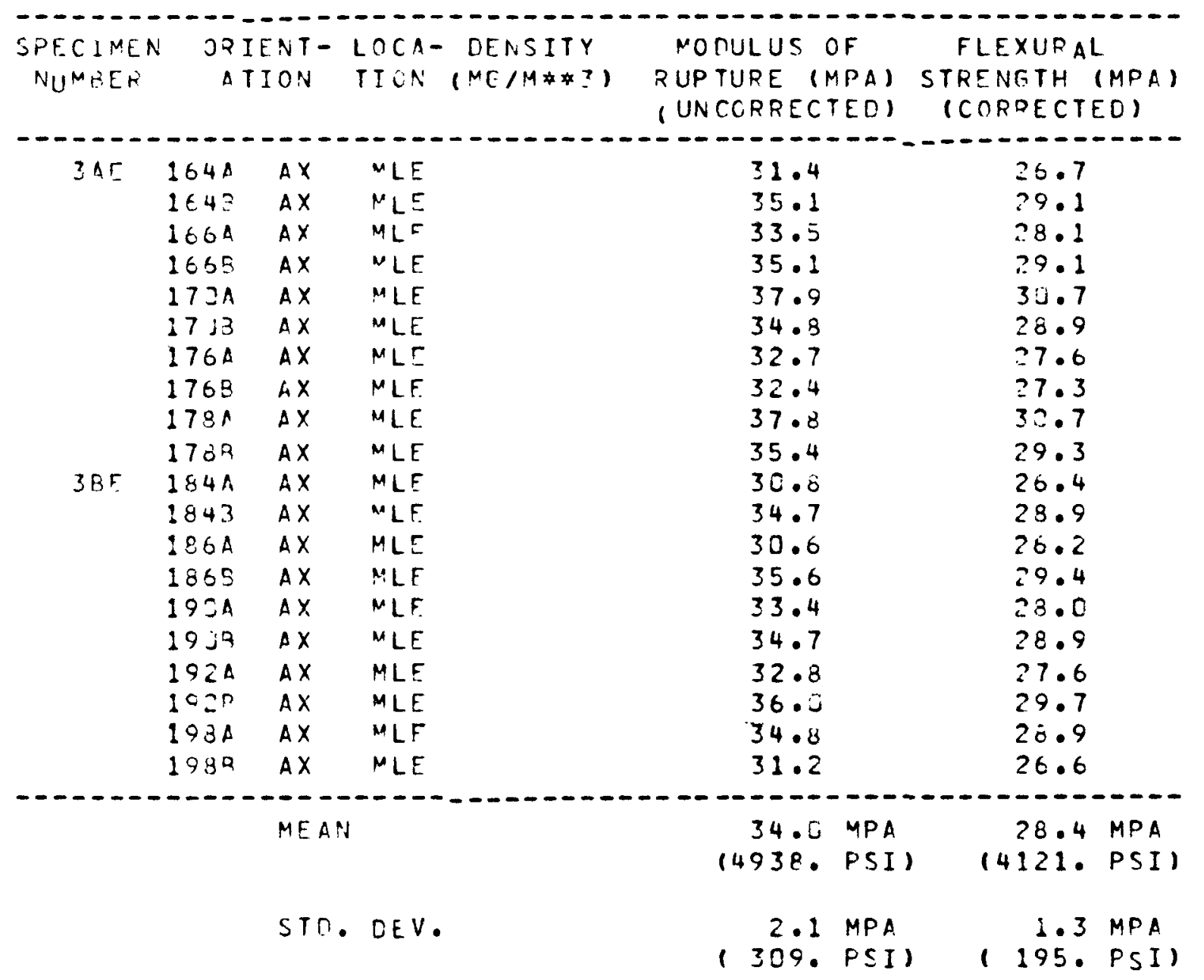


TABLE B-6 (Continued)

FIEXUFAL PRCPLRTIES OF H-451 RRAPHITE

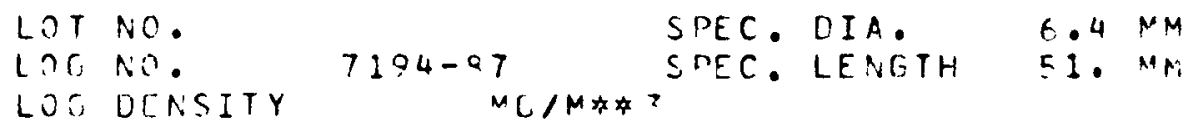

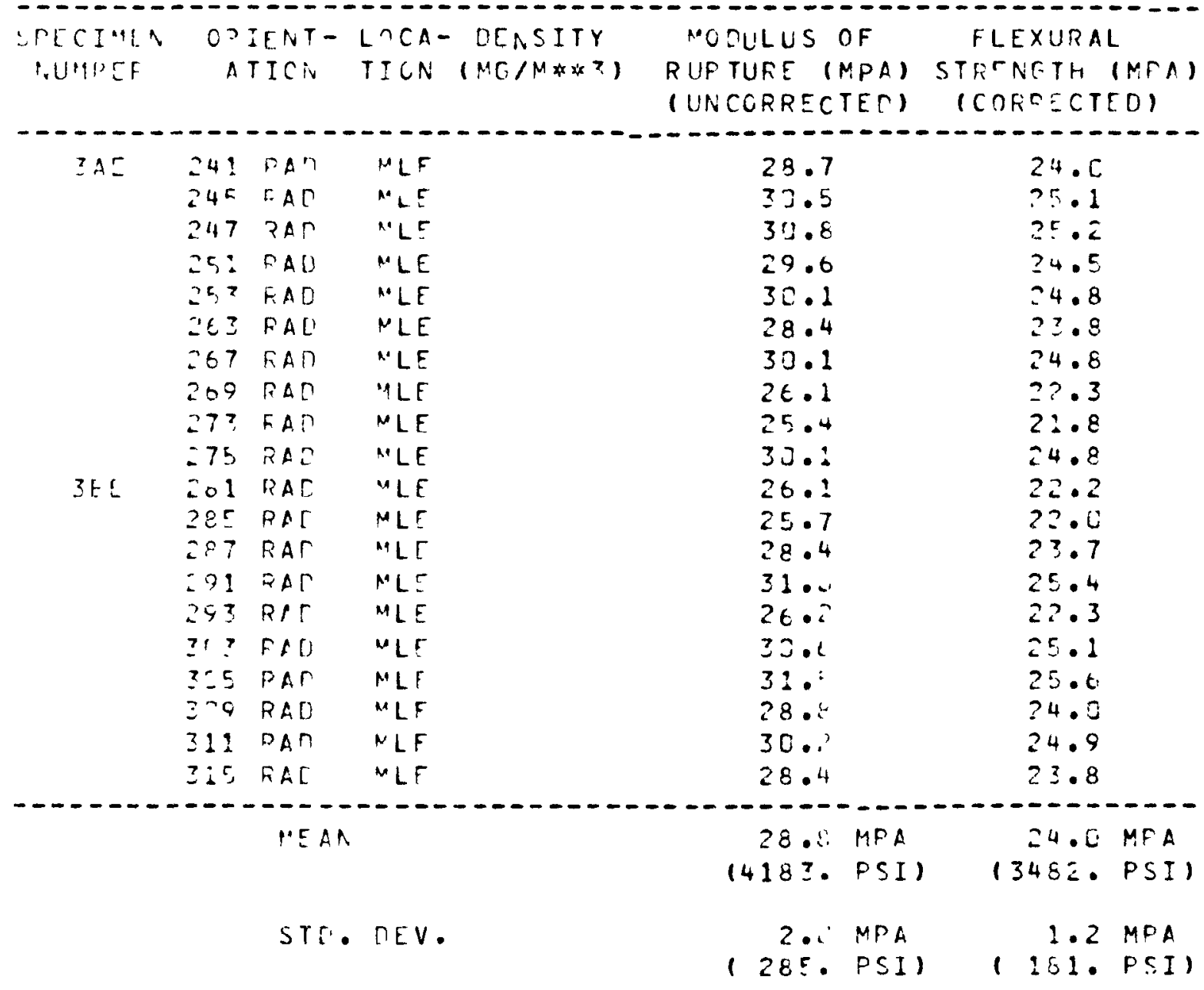


TABLE B-7

COMPRESSIVE PROFERTIES OF H-451 GRAPHITE

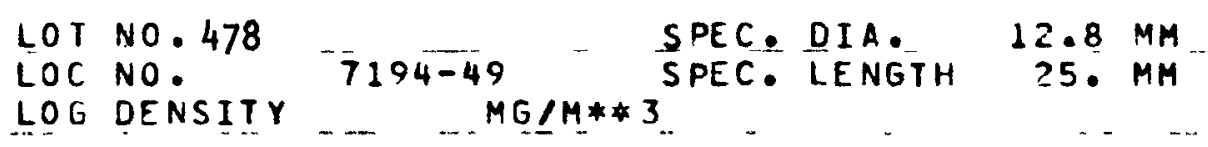

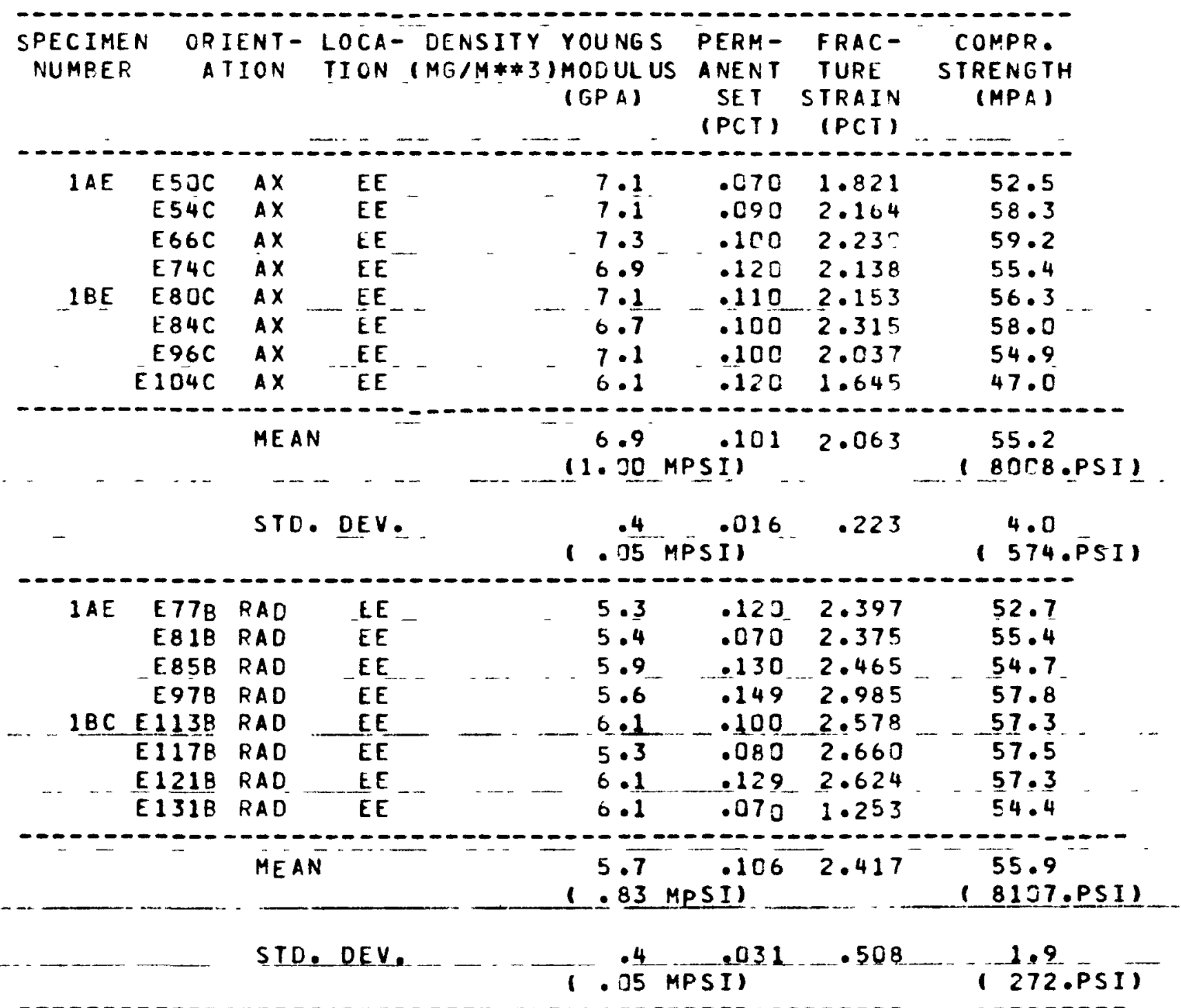


TABLE B-7 (Continued)

COMPRESSIVE PROPERTIES OF H-451 GRAPHITE

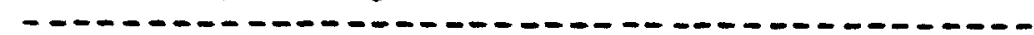

$\begin{array}{lllr}\text { LOI NO. } 478 & & \text { SPEC. DIA. } & 12.8 \mathrm{MM} \\ \text { LOG NO. } & 7194-49 & \text { SPEC. LENGTH } & 25 . \\ \text { LOG DENSITY } & \text { ME/M\#\# } & \end{array}$

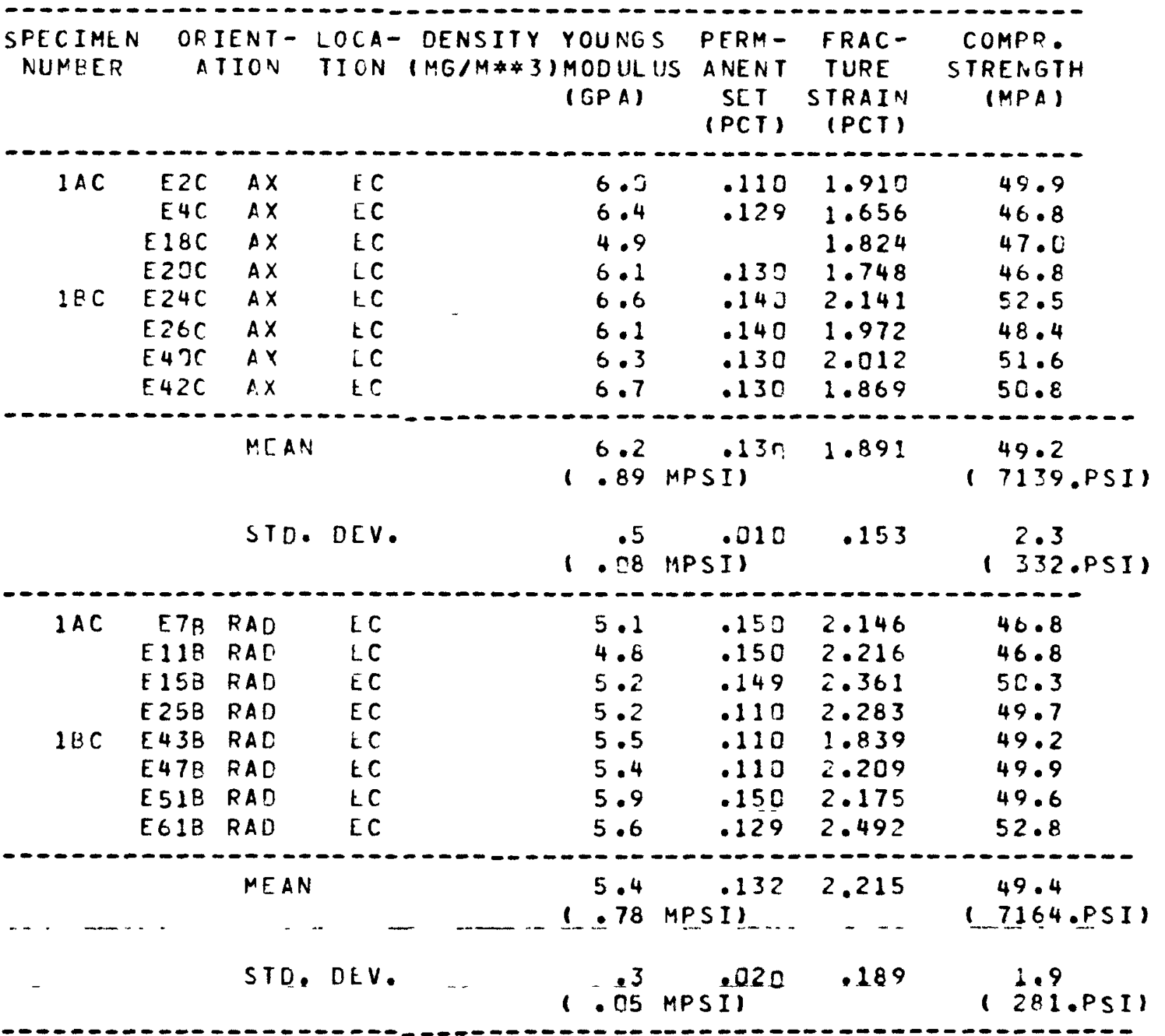


TABLE B-7 (Continued)

COMPRESSIVE PFOPEFTIES OF H-451 GRAPHITC

LOT NO.478
LOG NO. SPEC. OIA.
LOC DENSITY
LOS

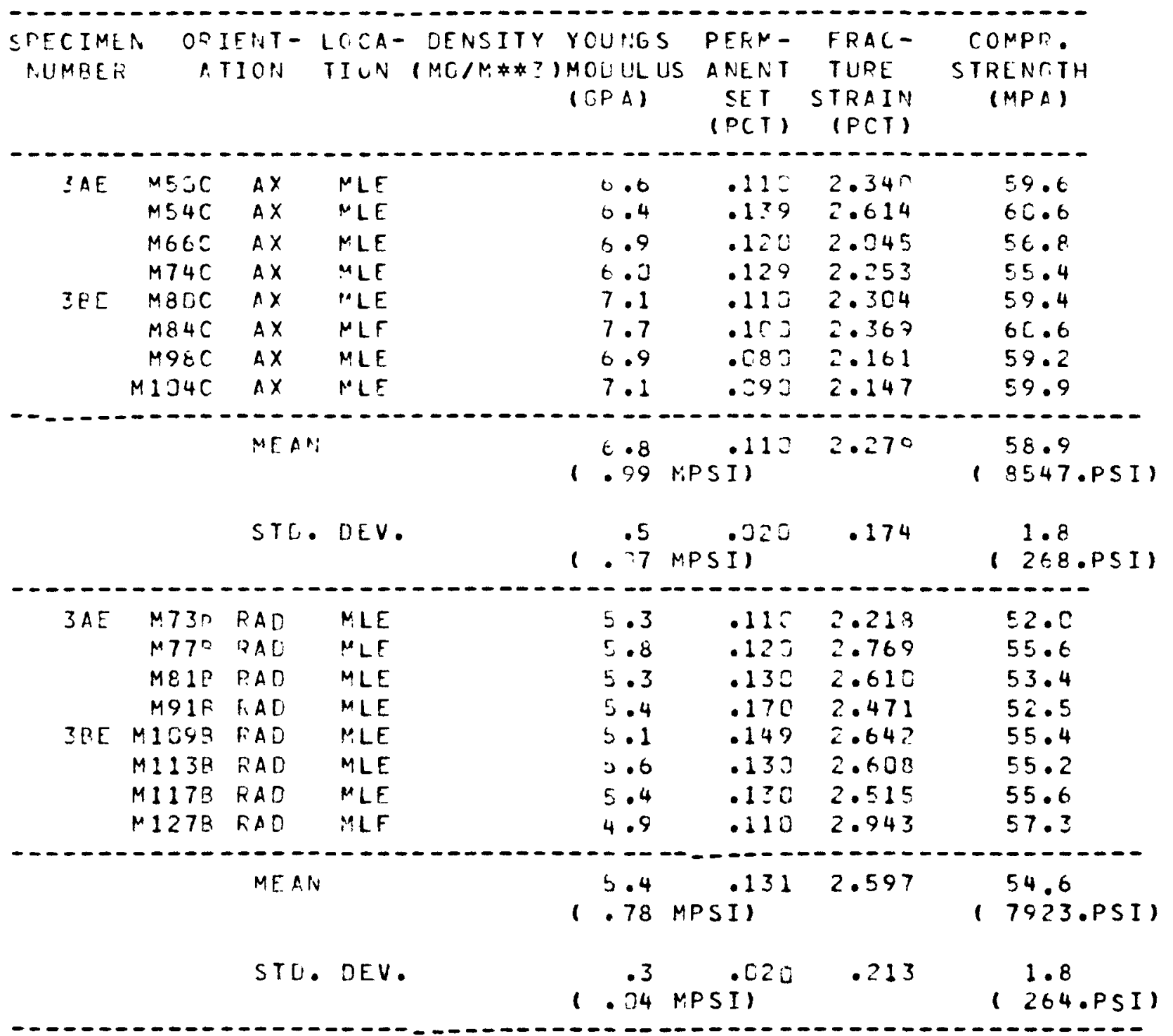


TABLE B-7 (Continued)

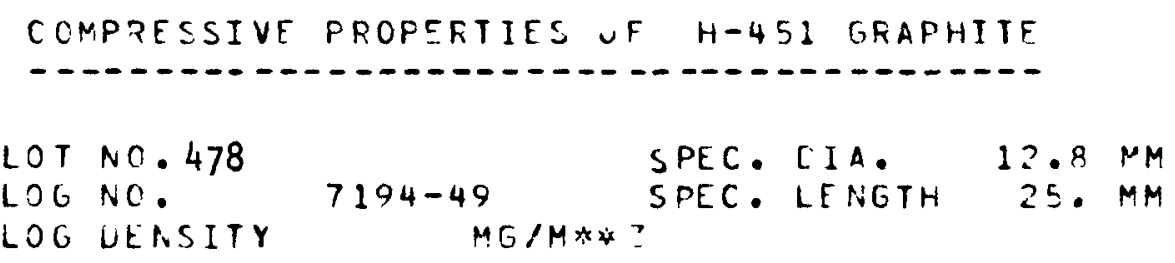

\begin{tabular}{|c|c|c|c|c|c|c|c|c|}
\hline $\begin{array}{l}\text { SPECIMEN } \\
\text { NUMQEF }\end{array}$ & \multicolumn{2}{|c|}{$\begin{array}{l}\text { ORIENT- } \\
\text { ATION }\end{array}$} & $\begin{array}{l}\text { LOCA- } \\
\text { TICN }\end{array}$ & $\begin{array}{l}\text { UENSITY } \\
(N G / M * 3)\end{array}$ & $\begin{array}{l}\text { YOURCS } \\
\text { IMOQULUS } \\
\text { (OPA) }\end{array}$ & $\begin{array}{c}\text { PERM- } \\
\text { S ANENT } \\
\text { SET } \\
(P C T)\end{array}$ & $\begin{array}{l}\text { FRAC- } \\
\text { TURE } \\
\text { STRAIN } \\
\text { (PCT) }\end{array}$ & $\begin{array}{l}\text { COMPE } \\
\text { STRENGTH } \\
\text { (MFA) }\end{array}$ \\
\hline---- & 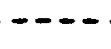 & $\ldots-$ & -5 & & $\cdots-$ & ----- & 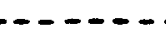 & $\cdots-\cdots$ \\
\hline \multirow[t]{4}{*}{$3 A C$} & $M=C$ & $A x$ & $M L C$ & & 0.3 & .120 & 2.035 & 50.8 \\
\hline & $M 4 C$ & $A X$ & $M L C$ & & 6.4 & .120 & $1.8 \geq 5$ & 51.3 \\
\hline & $M 18 \mathrm{C}$ & $A X$ & $M L C$ & & 3.6 & -1こi & 1.907 & $4 \dot{\varepsilon} \cdot 2$ \\
\hline & $M 20 C$ & $A X$ & $M L C$ & & 6.1 & $.14^{-}$ & 1.883 & 46.5 \\
\hline \multirow[t]{4}{*}{$3 B C$} & $M 24 C$ & $A X$ & MLC & & 0.6 & $.11=$ & 2.057 & $54 . \hat{I}$ \\
\hline & $M 2 \in C$ & $A X$ & $M L C$ & & 0.4 & .140 & 1.833 & 45.7 \\
\hline & $M 4=C$ & $A X$ & MLC & & $6 \cdot 3$ & .119 & 1.987 & 51.3 \\
\hline & 1920 & $A x$ & $M L C$ & & $t \cdot 3$ & .110 & 2.712 & 53.4 \\
\hline \multirow{2}{*}{\multicolumn{5}{|c|}{$\begin{array}{r}\text { MEAN } \\
\text { ME }\end{array}$}} & $--\infty$ & $\cdots$ & $\cdots---$ & $-\cdots$ \\
\hline & & & & & $\begin{array}{l}6.0 \\
.87 \mathrm{M}\end{array}$ & MPSII & 1.938 & $\left(\begin{array}{l}50.3 \\
7203 . P S I)\end{array}\right.$ \\
\hline & & STL. & - DEV. & & $1.14 \mathrm{~N}$ & MPSTI & .291 & $\left(\begin{array}{l}2.3 \\
327 . P S I)\end{array}\right.$ \\
\hline \multicolumn{9}{|c|}{ 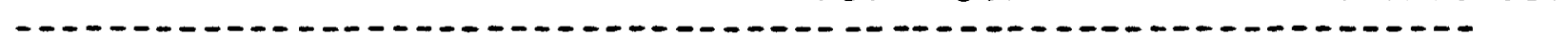 } \\
\hline \multirow[t]{4}{*}{$3 A C$} & 473 & FAO & MLC & & 5.4 & .132 & 2.407 & $51 \cdot 3$ \\
\hline & $M 12 \vec{R}$ & $2 A D$ & $M L C$ & & 5.2 & .172 & 2.379 & 52.3 \\
\hline & $M 15 \bar{\theta}$ & $2 A O$ & MLC & & 5.2 & .130 & 2.577 & 53.0 \\
\hline & $M 25 B$ & RAn & MLC & & 5.5 & .123 & $2.45 ?$ & 52.8 \\
\hline \multirow[t]{4}{*}{$3 B C$} & $M 433$ & RAD & $M L C$ & & $\leq .2$ & .170 & 2.549 & 52.0 \\
\hline & $M 47 B$ & PAD & $M L C$ & & 5.0 & .169 & 2.474 & 52.8 \\
\hline & $M 5 \perp B$ & PAD & $M L C$ & & 4.8 & .193 & 2.537 & $51 \cdot 1$ \\
\hline & $M 55 E$ & FAD & $M L C$ & & 5.1 & .142 & 2.714 & 53.9 \\
\hline \multirow{3}{*}{\multicolumn{5}{|c|}{$\begin{array}{r}\text { MEAN } \\
\cdots\end{array}$}} & & & & --- \\
\hline & & & & & 5.2 & .152 & 2.512 & 52.4 \\
\hline & & & & & $1.75 \mathrm{M}$ & MPS I I & & 17595 \\
\hline & & STL. & - DEV & & $1.22 \mathrm{M}$ & MPSII & .107 & $(1 \dot{3} 6$. PSI \\
\hline
\end{tabular}


TABLE B-8

COMPRESSIVE PROPERTIES OF H-451 GRAPHITE

- - - - - - - - - - - - - - - - - - - - - -
LOT NO. 478
SPEC. DIA.
$12.9 \mathrm{MM}$
LOG NO.
$7194-52$
SPEC • LENGTH
25. $M M$
LOC DENSITY $M G / M * 3$

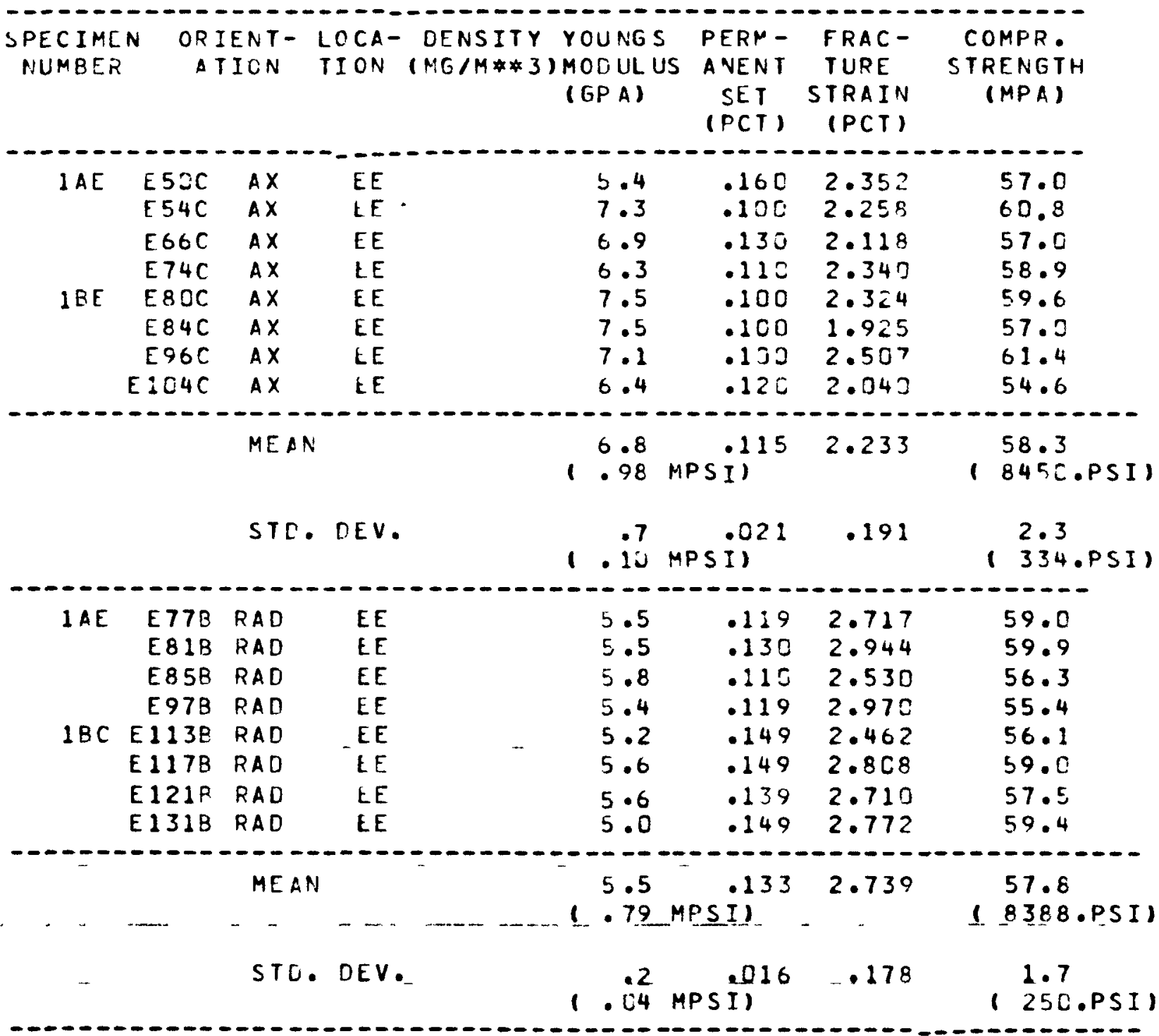


TABLE B-8 (Continued)

CIMPRESSIVE PROPERTIES $\checkmark r$ H-451 GRAPHITE

$\begin{array}{lllr}\text { LOT NO.478 } & & \text { SPEC. OIA. } & 12.8 \mathrm{MM} \\ \text { LO, NO. } & 7194-52 & \text { SPEC. LENGTH } & 25 . \mathrm{MM}\end{array}$

LO, NO.

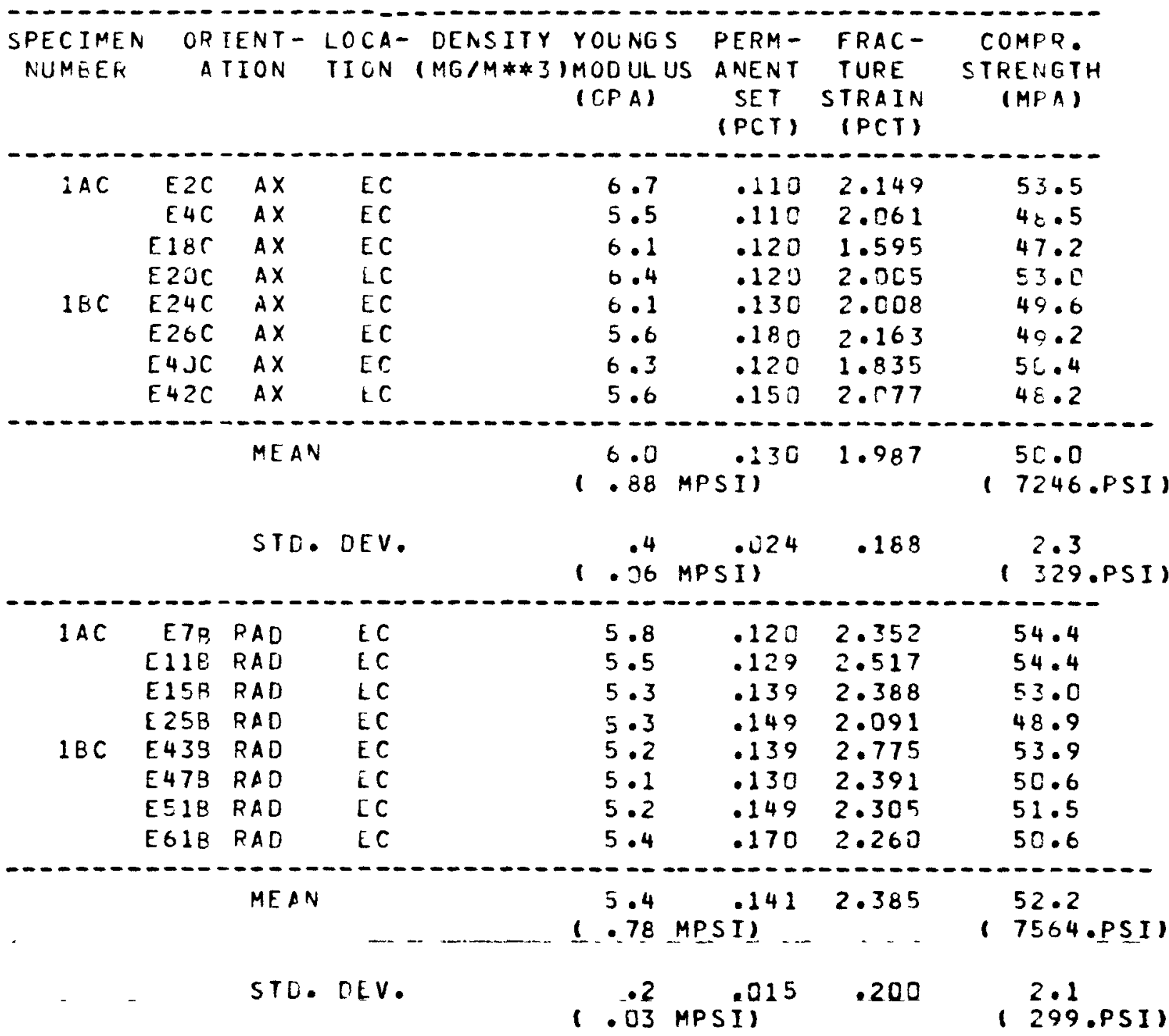


TABLE B-8 (Continued)

COMPRESSIV̄E PROPERTIES OF H-45i GRAPHITE

COMPRESSIVE PROPERT IES OF H

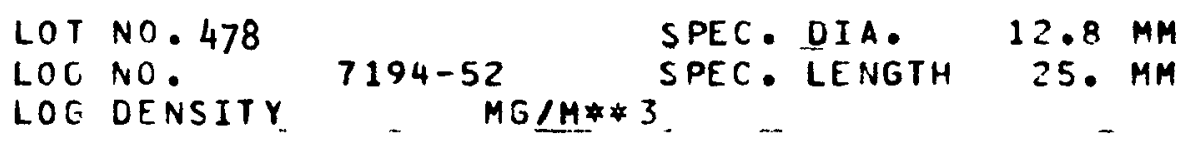

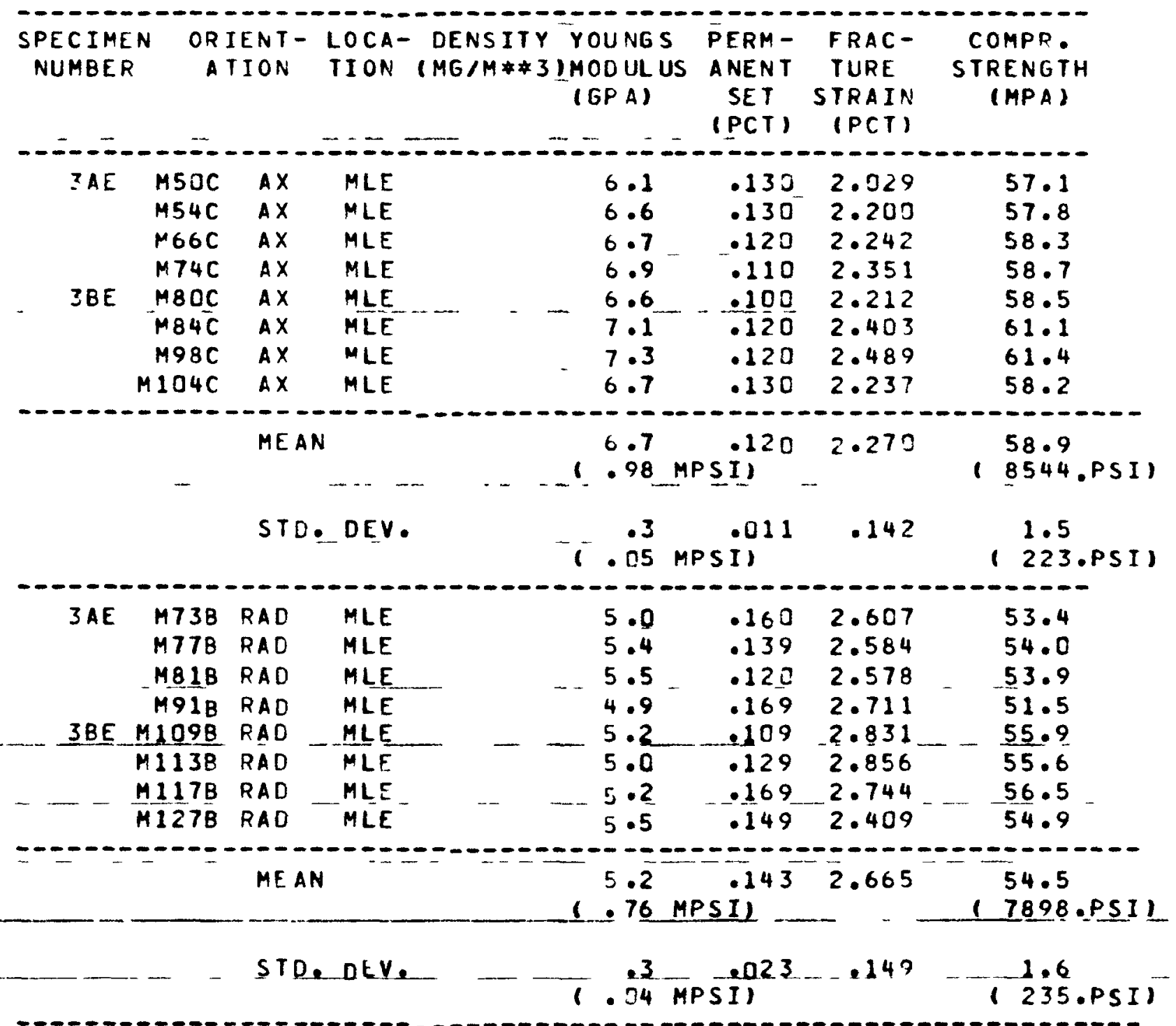


TABLE B-8 (Continued)

COMPRESSIVE PROPERTIES OF H-451 GRAPHITE

COMPRESSI

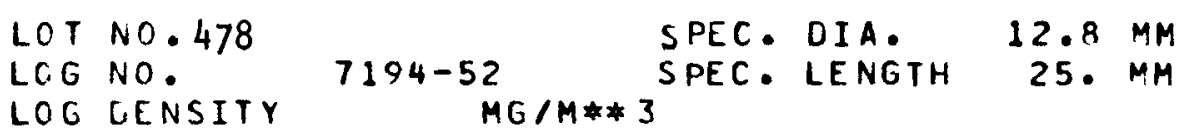

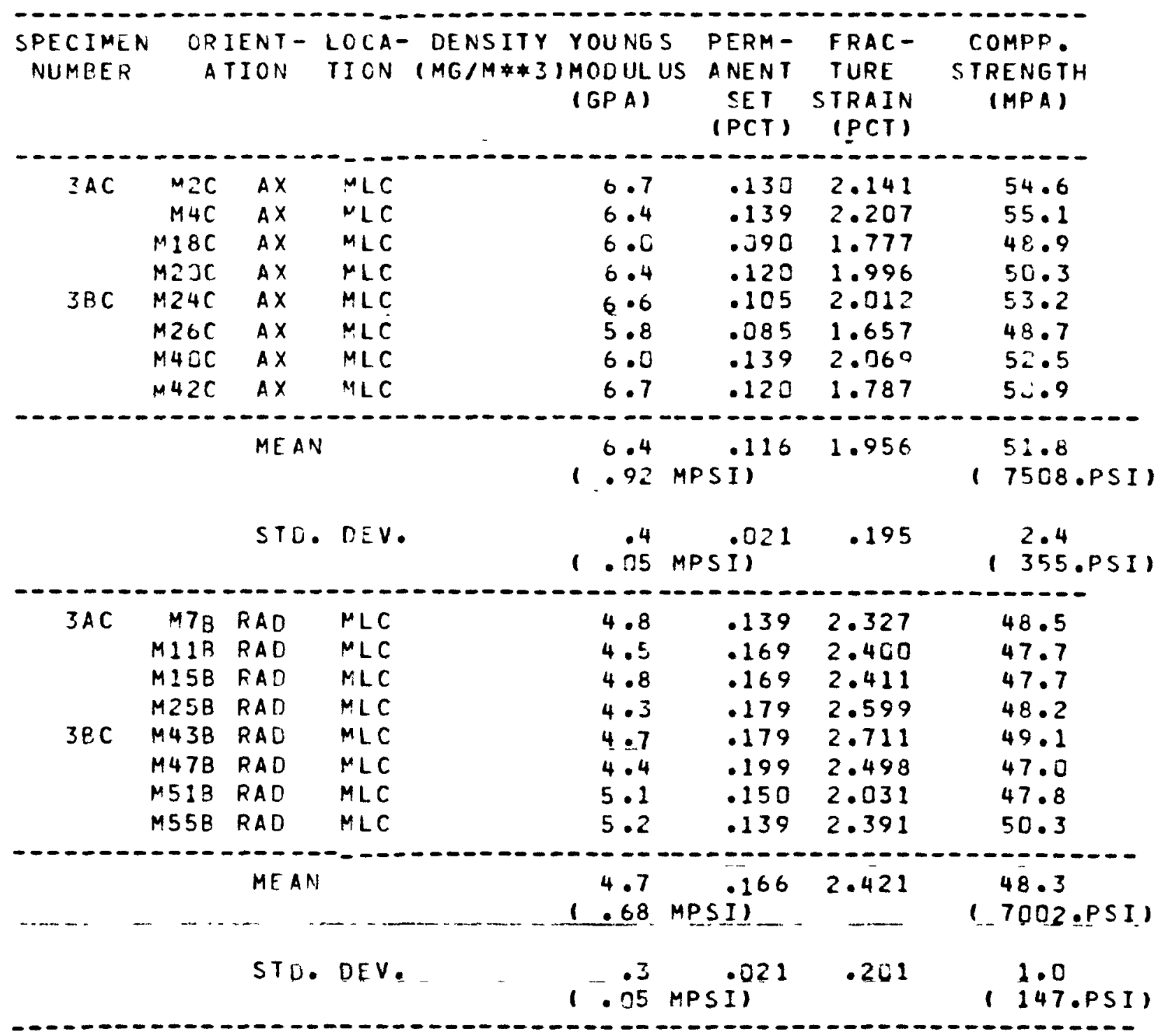


TABLE B-9

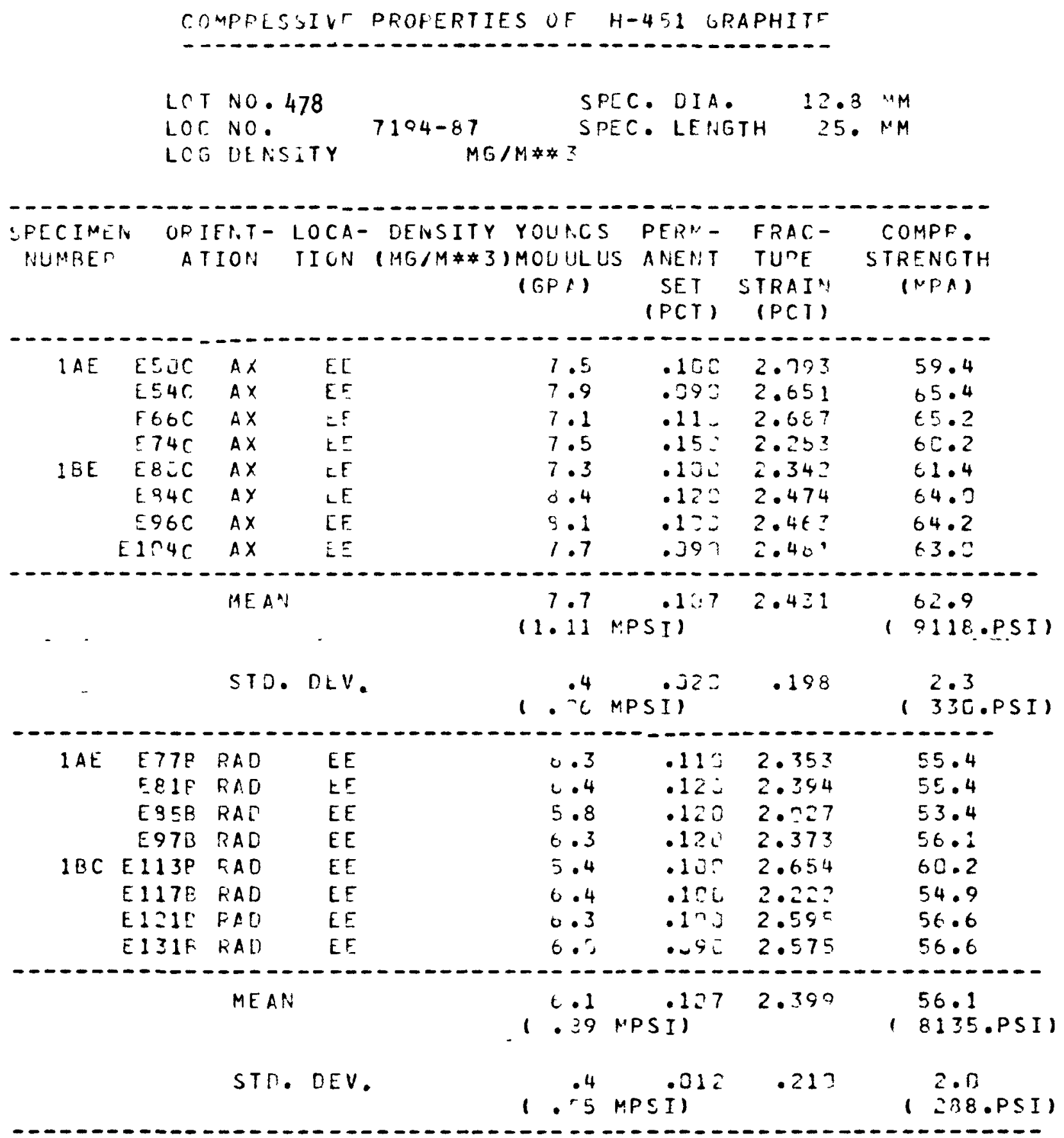


TABLE B-9 (Continued)

COMPFESSIVE PROPERTIES OF H-451 GRAPHITE

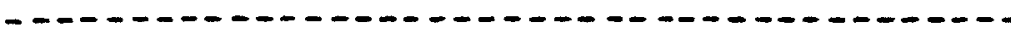

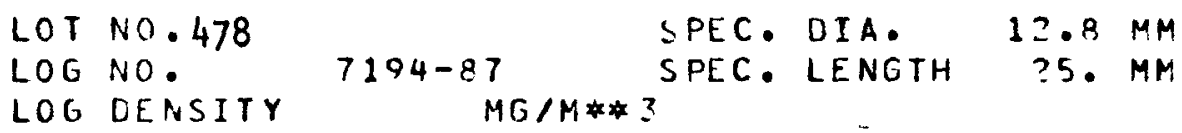

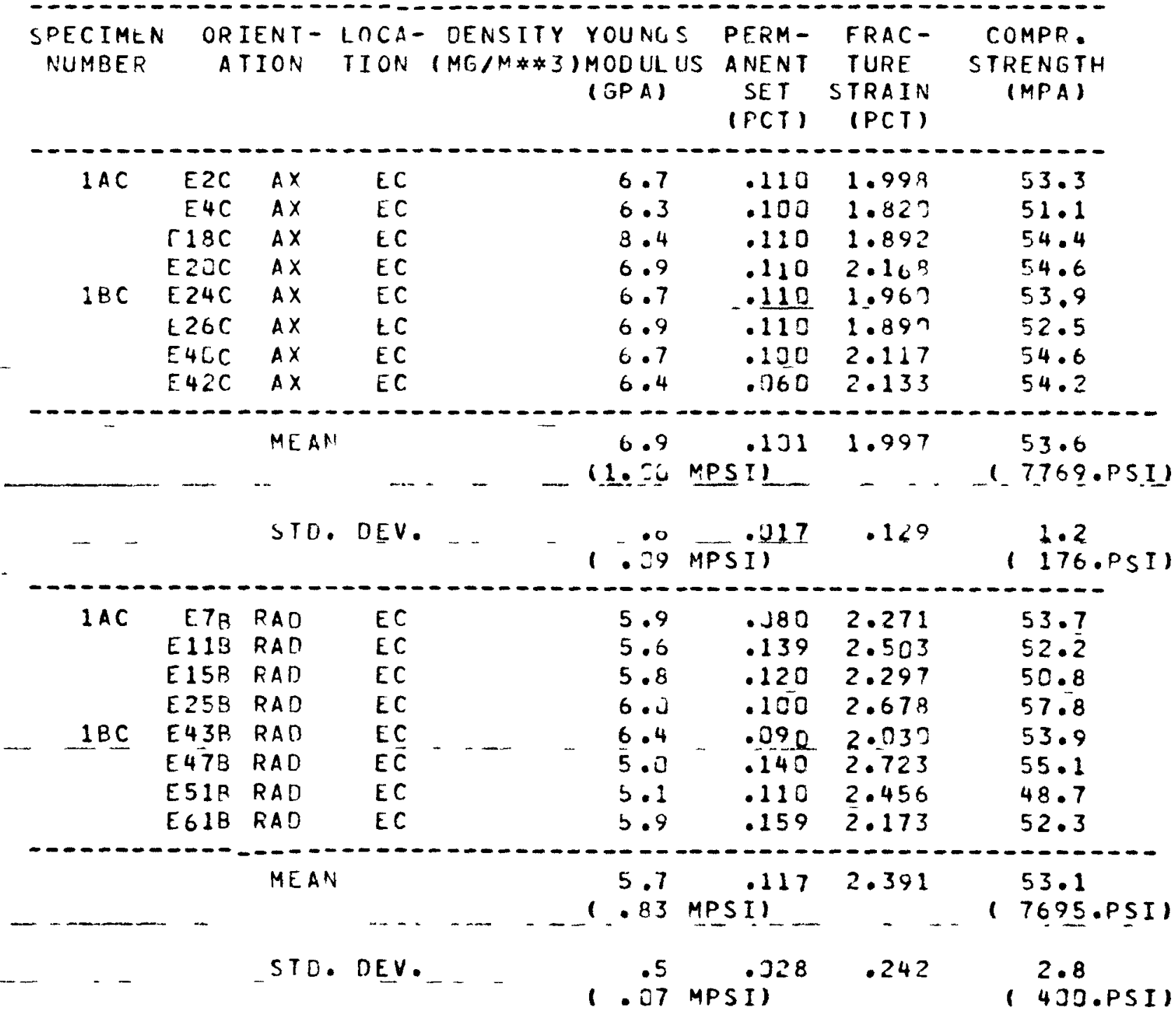


TABLE B-9 (Continued)

COMPRECSIVE TSOPERTIES UF H-4T1 GRAFHITE

$\begin{array}{llll}\text { LOT } N 0.478 & \text { SPEC. OIA. } & 12.3 \mathrm{MM} \\ \text { LOG NO. } & 7294-87 & \text { SPEC. LETGTH T.5. MM }\end{array}$ MG/Pi* 3

LOE DERSITY

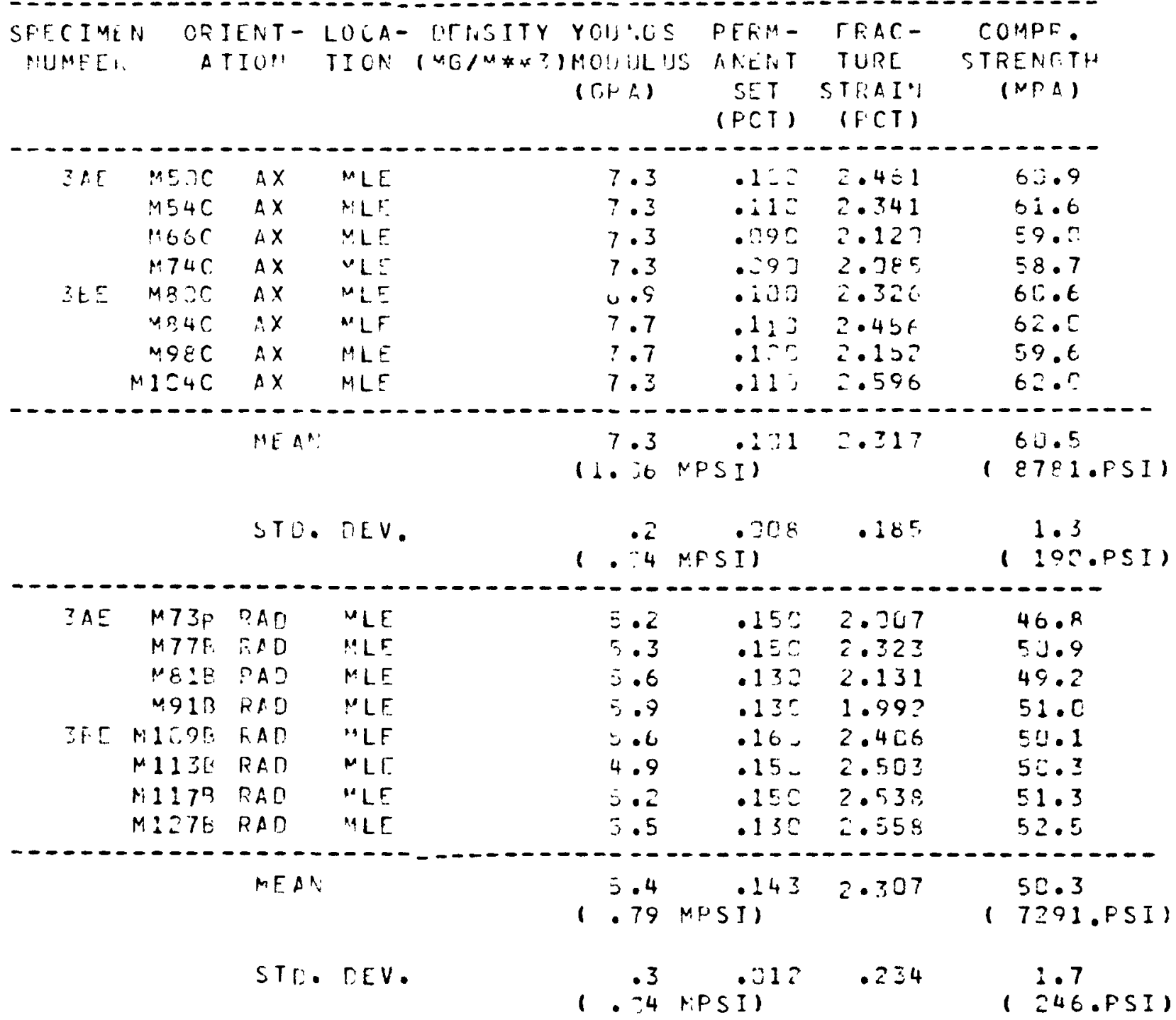


TABLE B-9 (Continued)

COMPRESSILE PROPTRTIES UF H-4EI GFAPHIT

-

LOT NO. 478
IOG NO.
LOC LENSITY

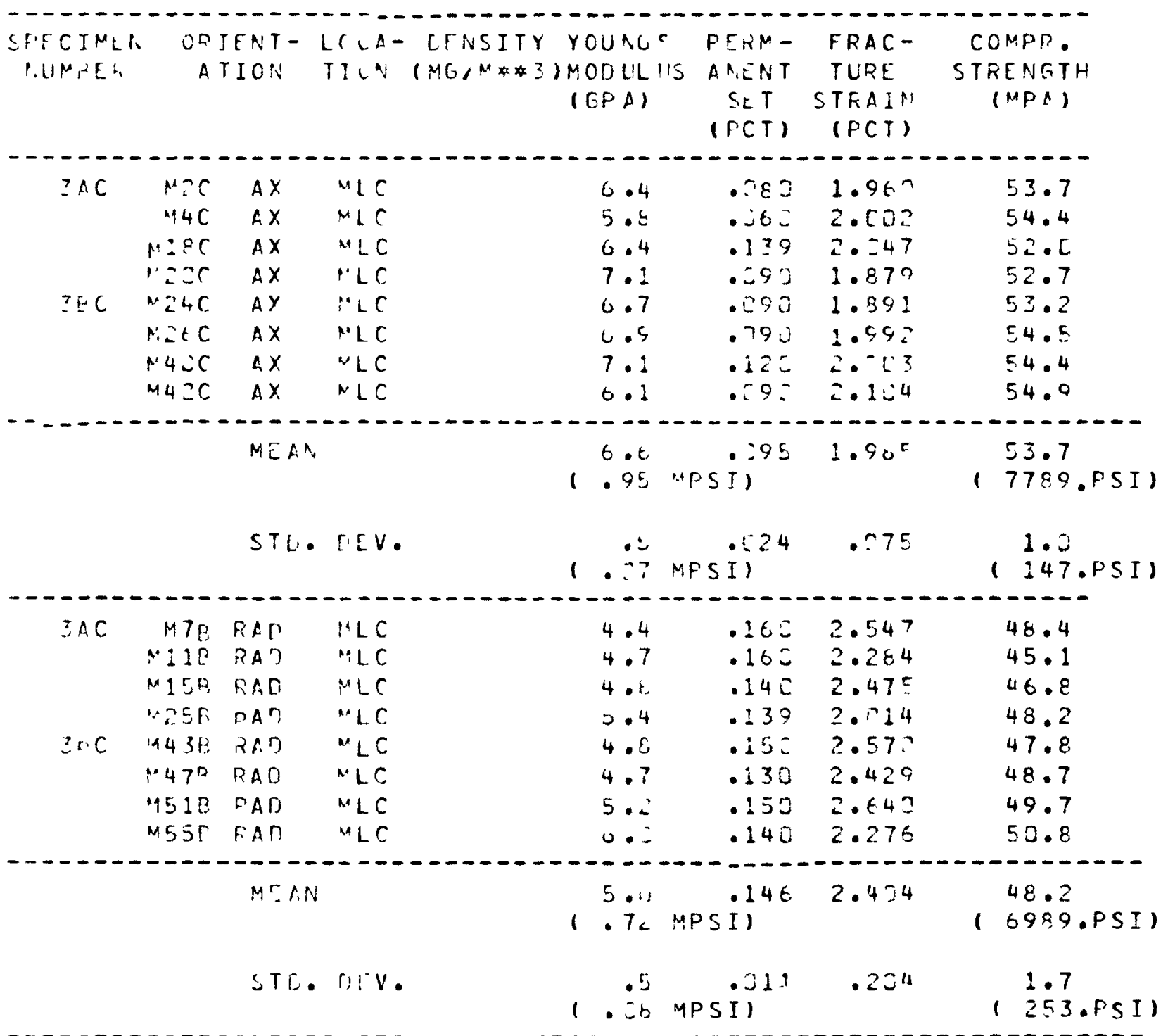


TABLE B-10

THERMAL EXPANSIVITY OF H-451 GRAPHITE

LOG 7194-49

\begin{tabular}{|c|c|c|c|c|}
\hline $\begin{array}{l}\text { Specimen } \\
\text { Number }\end{array}$ & Orientation & Location & $\begin{array}{l}\bar{\alpha}_{\mathrm{RT}-773 \mathrm{~K}} \\
\left(10^{-6} \mathrm{~K}^{-1}\right)\end{array}$ & $\begin{array}{l}\bar{\alpha}_{\mathrm{RT}-1173 \mathrm{~K}} \\
\left(10^{-6} \mathrm{~K}^{-1}\right)\end{array}$ \\
\hline $1 \mathrm{AE}-62 \mathrm{~A}$ & $A x$ & $\mathrm{EE}$ & 3.79 & 4.25 \\
\hline$-62 B$ & $A x$ & $\mathrm{EE}$ & 3.81 & 4.28 \\
\hline$-72 \mathrm{~A}$ & $\mathrm{Ax}$ & $\mathrm{EE}$ & 3.75 & 4.24 \\
\hline$-72 B$ & $A x$ & $\mathrm{EE}$ & 3.75 & 4.19 \\
\hline \multicolumn{3}{|c|}{ Mean } & 3.78 & 4.24 \\
\hline \multicolumn{3}{|c|}{ Std. dev. } & 0.03 & 0.04 \\
\hline $2 A C-108 A$ & $A x$ & MLC & 3.62 & 4.15 \\
\hline$-108 B$ & $A x$ & MLC & 3.69 & 4.16 \\
\hline$-118 A$ & $\mathrm{Ax}$ & MLC & 3.80 & 4.27 \\
\hline$-118 \mathrm{~B}$ & $\mathrm{Ax}$ & MLC & 3.76 & 4.24 \\
\hline \multicolumn{3}{|c|}{ Mean } & 3.72 & 4.21 \\
\hline \multicolumn{3}{|c|}{ Std. dev. } & 0.08 & 0.06 \\
\hline $1 \mathrm{AE}-83 \mathrm{~A}$ & $\operatorname{Rad}$ & $\mathrm{EE}$ & 4.51 & 4.95 \\
\hline$-8 \cdot 3 B$ & $\operatorname{Rad}$ & $\mathrm{EE}$ & 4.56 & 4.92 \\
\hline$-107 A$ & Rad & $\mathrm{EE}$ & 4.57 & 5.03 \\
\hline$-107 \mathrm{~B}$ & $\operatorname{Rad}$ & $\mathrm{EE}$ & 4.42 & 4.86 \\
\hline \multicolumn{3}{|c|}{ Mean } & 4.52 & 4.94 \\
\hline \multicolumn{3}{|c|}{ Std. dev. } & 0.07 & 0.07 \\
\hline $3 A C-169 A$ & $\operatorname{Rad}$ & MLC & 4.14 & 4.76 \\
\hline$-169 \mathrm{~B}$ & $\operatorname{Rad}$ & MLC & 4.25 & 4.80 \\
\hline$-187 A$ & $\operatorname{Rad}$ & MLC & 4.35 & 4.82 \\
\hline$-187 \mathrm{~B}$ & $\operatorname{Rad}$ & MLC & 4.34 & 4.80 \\
\hline \multicolumn{3}{|c|}{ Mean } & 4.27 & 4.80 \\
\hline \multicolumn{3}{|c|}{ Std. dev. } & 0.10 & 0.03 \\
\hline
\end{tabular}


TABLE B-11

THERMAL EXPANSIVITY OF H-451 GRAPHITE

LOG $7194-52$

\begin{tabular}{|c|c|c|c|c|}
\hline $\begin{array}{l}\text { Specimen } \\
\text { Number }\end{array}$ & Orientation & Location & $\begin{array}{l}\bar{\alpha}_{(\mathrm{RT}-773 \mathrm{~K})} \\
\left(10^{-6} \mathrm{~K}^{-1}\right)\end{array}$ & $\begin{array}{l}\bar{\alpha}_{(\mathrm{RT}-1173 \mathrm{~K})} \\
\left(10^{-6} \mathrm{~K}^{-1}\right)\end{array}$ \\
\hline $1 \mathrm{AC}-6 \mathrm{~A}$ & $\mathrm{Ax}$ & EC & 3.83 & 4.37 \\
\hline$-6 B$ & $\mathrm{Ax}$ & EC & 3.80 & 4.35 \\
\hline$-18 A$ & $\mathrm{Ax}$ & $\mathrm{EC}$ & 3.71 & 4.22 \\
\hline$-18 B$ & $\mathrm{Ax}$ & EC & 3.78 & 4.29 \\
\hline $1 \mathrm{BC}-34 \mathrm{~A}$ & $\mathrm{Ax}$ & EC & 3.84 & 4.27 \\
\hline$-34 B$ & $\mathrm{Ax}$ & EC & 3.90 & 4.40 \\
\hline$-48 \mathrm{~A}$ & $\mathrm{Ax}$ & EC & 3.87 & 4.35 \\
\hline$-48 \mathrm{~B}$ & $A x$ & EC & 3.53 & 4.12 \\
\hline \multicolumn{3}{|c|}{ Mean } & 3.78 & 4.30 \\
\hline \multicolumn{3}{|c|}{ Std. dev. } & 0.12 & 0.09 \\
\hline $1 \mathrm{AE}-62 \mathrm{~A}$ & $\mathrm{Ax}$ & $\mathrm{EE}$ & 3.68 & 4.13 \\
\hline$-62 B$ & $A x$ & $\mathrm{EE}$ & 3.73 & 4.18 \\
\hline$-72 \mathrm{~A}$ & $\mathrm{Ax}$ & $\mathrm{EE}$ & 3.87 & 4.20 \\
\hline$-72 B$ & $\mathrm{Ax}$ & $\mathrm{EE}$ & 3.97 & 4.43 \\
\hline $1 \mathrm{BE}-82 \mathrm{~A}$ & $\mathrm{Ax}$ & $\mathrm{EE}$ & 3.68 & 4.16 \\
\hline $82 B$ & $\mathrm{Ax}$ & $\mathrm{EE}$ & 4.32 & 4.47 \\
\hline $92 \mathrm{~A}$ & $\mathrm{Ax}$ & $\mathrm{EE}$ & 4.24 & $4 \cdot 30$ \\
\hline $92 \mathrm{~B}$ & $A x$ & $\mathrm{EE}$ & 4.42 & 4.59 \\
\hline \multicolumn{3}{|c|}{ Mean } & 3.99 & 4.31 \\
\hline \multicolumn{3}{|c|}{ Std. dev. } & 0.30 & 0.17 \\
\hline
\end{tabular}


TABLE B-11 (Continued)

\begin{tabular}{|c|c|c|c|c|}
\hline $\begin{array}{l}\text { Specimen } \\
\text { Number }\end{array}$ & Orientation & Location & $\begin{array}{l}{ }^{\bar{\alpha}}(\mathrm{RT}-773 \mathrm{~K}) \\
\quad\left(10^{-6} \mathrm{~K}^{-1}\right)\end{array}$ & $\begin{array}{r}\bar{\alpha}(\mathrm{RT}-1173 \mathrm{~K}) \\
\left(10^{-6} \mathrm{~K}^{-1}\right)\end{array}$ \\
\hline $3 A C-108 A$ & $\mathrm{Ax}$ & MLC & 3.39 & 4.26 \\
\hline$-108 \mathrm{~B}$ & $\mathrm{Ax}$ & MLC & 3.73 & 4.18 \\
\hline$-118 A$ & $A x$ & MLC & 3.76 & 4.21 \\
\hline$-118 \mathrm{~B}$ & $\mathrm{Ax}$ & MLC & 3.68 & 4.16 \\
\hline $3 B C-134 A$ & $\mathrm{Ax}$ & MLC & 3.63 & 4.19 \\
\hline$-134 B$ & $\mathrm{Ax}$ & MLC & 3.80 & 4.26 \\
\hline$-146 A$ & $\mathrm{Ax}$ & MLC & 3.51 & 4.14 \\
\hline$-146 B$ & $\mathrm{Ax}$ & MLC & 3.49 & 4.11 \\
\hline & Mean & & 3.62 & 4.19 \\
\hline & Std. dev. & & 0.15 & 0.05 \\
\hline $3 \mathrm{AE}-162 \mathrm{~A}$ & $A x$ & MLE & 3.82 & 4.21 \\
\hline$-162 B$ & $A x$ & MLE & 3.71 & 4.15 \\
\hline$-172 A$ & $A x$ & MLE & 3.75 & 4.21 \\
\hline$-172 B$ & $A x$ & MLE & 3.69 & 4.16 \\
\hline $3 \mathrm{BE}-182 \mathrm{~A}$ & $\mathrm{Ax}$ & MLE & 3.85 & 4.29 \\
\hline$-182 B$ & $A x$ & MLE & 3.65 & 4.16 \\
\hline$-192 \mathrm{~A}$ & $A x$ & MLE & 3.72 & 4.21 \\
\hline$-192 B$ & $A x$ & MLE & 3.66 & 4.21 \\
\hline & Mean & & 3.73 & 4.20 \\
\hline & Std. dev. & & 0.07 & 0.05 \\
\hline $1 \mathrm{AC}-9 \mathrm{~A}$ & $\operatorname{Rad}$ & EC & 4.54 & 4.94 \\
\hline$-9 B$ & $\operatorname{Rad}$ & $\mathrm{EC}$ & 4.32 & 4.80 \\
\hline$-27 A$ & $\operatorname{Rad}$ & $\mathrm{EC}$ & 4.42 & 4.90 \\
\hline$-27 B$ & $\operatorname{Rad}$ & $\mathrm{EC}$ & 4.58 & 4.96 \\
\hline $1 \mathrm{BC}-49 \mathrm{~A}$ & Rad & $\mathrm{EC}$ & 4.50 & 4.99 \\
\hline$-49 B$ & $\operatorname{Rad}$ & $\mathrm{EC}$ & 4.40 & 4.90 \\
\hline$-67 A$ & Rad & $\mathrm{EC}$ & 4.54 & 4.98 \\
\hline$-67 B$ & $\operatorname{Rad}$ & $\mathrm{EC}$ & 4.33 & 4.63 \\
\hline \multicolumn{3}{|c|}{ Mean } & 4.46 & 4.89 \\
\hline \multicolumn{3}{|c|}{ Std. dev. } & 0.10 & 0.12 \\
\hline
\end{tabular}


TABLE B-11 (Continued)

\begin{tabular}{|c|c|c|c|c|}
\hline $\begin{array}{l}\text { Specimen } \\
\text { Number }\end{array}$ & Orientation & Location & $\begin{array}{l}\bar{\alpha}_{(\mathrm{RT}-773 \mathrm{~K})} \\
\quad\left(10^{-6} \mathrm{~K}^{-1}\right)\end{array}$ & $\begin{array}{r}{ }^{\bar{\alpha}}(\mathrm{RT}-1173 \mathrm{~K}) \\
\left(10^{-6} \mathrm{~K}^{-1}\right)\end{array}$ \\
\hline $1 \mathrm{AE}-83 \mathrm{~A}$ & Rad & $\mathrm{EE}$ & 5.08 & 5.49 \\
\hline$-83 B$ & $\operatorname{Rad}$ & $\mathrm{EE}$ & 4.57 & 5.08 \\
\hline$-107 \mathrm{~A}$ & $\operatorname{Rad}$ & $\mathrm{EE}$ & 4.76 & 5.29 \\
\hline$-107 \mathrm{~B}$ & $\operatorname{Rad}$ & $\mathrm{EE}$ & 4.54 & 5.08 \\
\hline $1 \mathrm{BE}-123 \mathrm{~A}$ & $\operatorname{Rad}$ & EE & 4.74 & 5.23 \\
\hline$-123 B$ & Rad & $\mathrm{EE}$ & 4.49 & 5.00 \\
\hline$-147 \mathrm{~A}$ & $\operatorname{Rad}$ & EE & 4.74 & 5.27 \\
\hline$-147 \mathrm{~B}$ & $\operatorname{Rad}$ & $\mathrm{EE}$ & 4.65 & 5.12 \\
\hline \multicolumn{3}{|c|}{ Mean } & 4.70 & 5.20 \\
\hline \multicolumn{3}{|c|}{ Std. dev. } & 0.19 & 0.16 \\
\hline $3 A C-169 A$ & Rad & MLC & 4.44 & 4.90 \\
\hline$-169 \mathrm{~B}$ & Rad & MLC & 4.40 & 4.87 \\
\hline$-187 A$ & Rad & MLC & 4.23 & 4.75 \\
\hline$-187 \mathrm{~B}$ & $\operatorname{Rad}$ & MLC & 4.50 & 4.93 \\
\hline $3 B C-209 A$ & $\operatorname{Rad}$ & MLC & 4.34 & 4.83 \\
\hline$-209 B$ & $\operatorname{Rad}$ & MLC & $4 \cdot 13$ & 4.66 \\
\hline$-227 A$ & $\operatorname{Rad}$ & MLC & 4.38 & 4.86 \\
\hline$-227 B$ & Rad & MLC & 4.40 & 4.83 \\
\hline \multicolumn{3}{|c|}{ Mean } & 4.35 & 4.83 \\
\hline \multicolumn{3}{|c|}{ Std. dev } & 0.12 & 0.09 \\
\hline $3 \mathrm{AE}-249 \mathrm{~A}$ & Rad & MLE & 4.36 & 4.83 \\
\hline$-249 B$ & Rad & MLE & 4.43 & 4.88 \\
\hline$-265 A$ & Rad & MLE & 4.64 & 4.99 \\
\hline$-265 B$ & Rad & MLE & 4.56 & 4.98 \\
\hline $3 B E-289 A$ & Rad & MLE & 4.54 & 4.99 \\
\hline$-289 B$ & $\operatorname{Rad}$ & MLE & 4.31 & 4.85 \\
\hline$-307 \mathrm{~A}$ & Rad & MLE & 3.77 & 4.65 \\
\hline$-307 B$ & $\mathrm{Rad}$ & MLE & 3.95 & 4.92 \\
\hline \multicolumn{3}{|c|}{ Mean } & 4.32 & 4.89 \\
\hline \multicolumn{3}{|c|}{ Std. dev. } & 0.31 & 0.11 \\
\hline
\end{tabular}


TABLE B-12

THEPMAL CORCUCTIVITY OF H-451 GRAPHITE

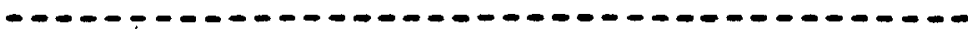

LCT NUMBE:

478

LOG NUMBEF:

$7194-49$

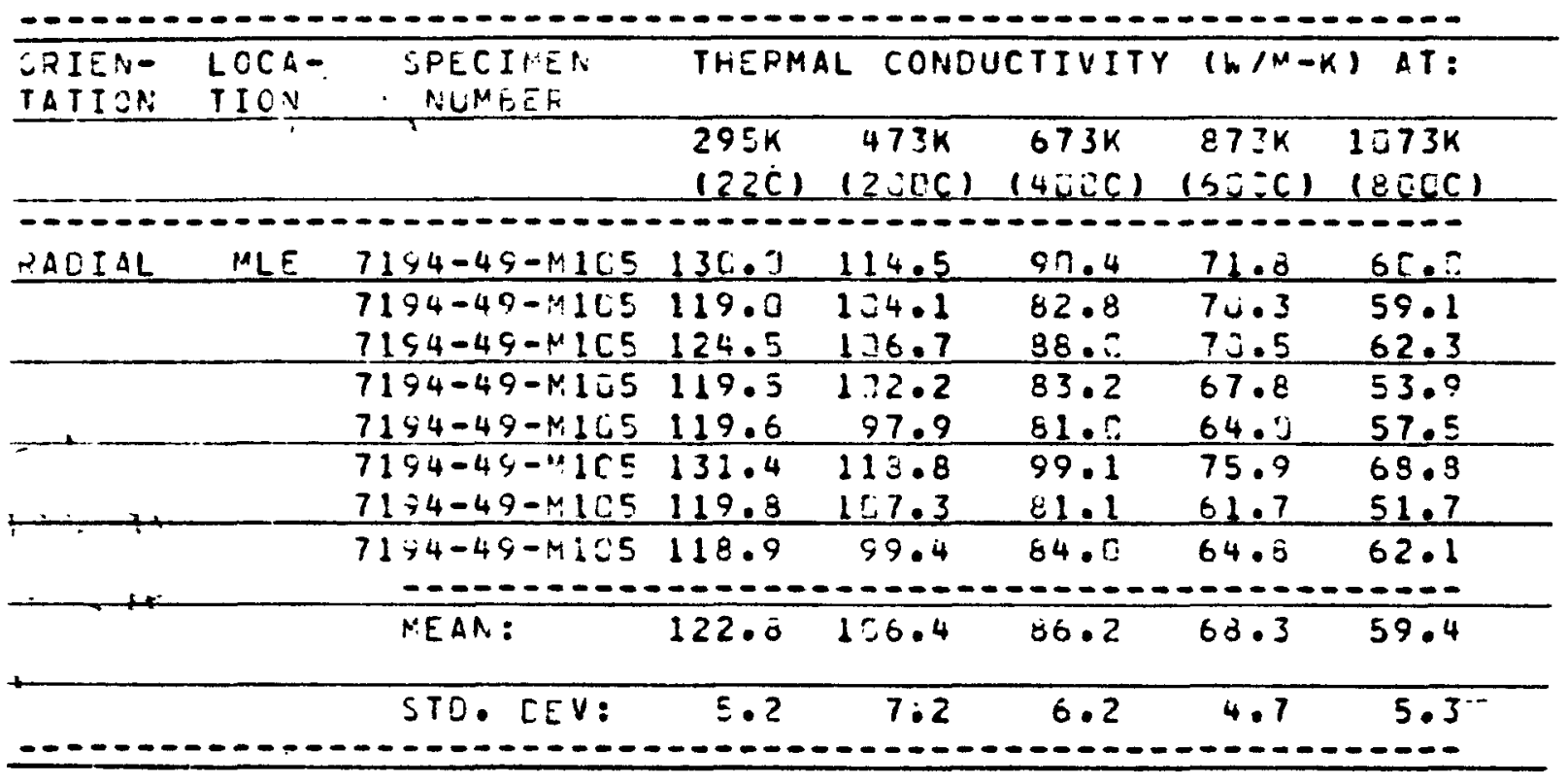


TABLE B-12 (Continued)

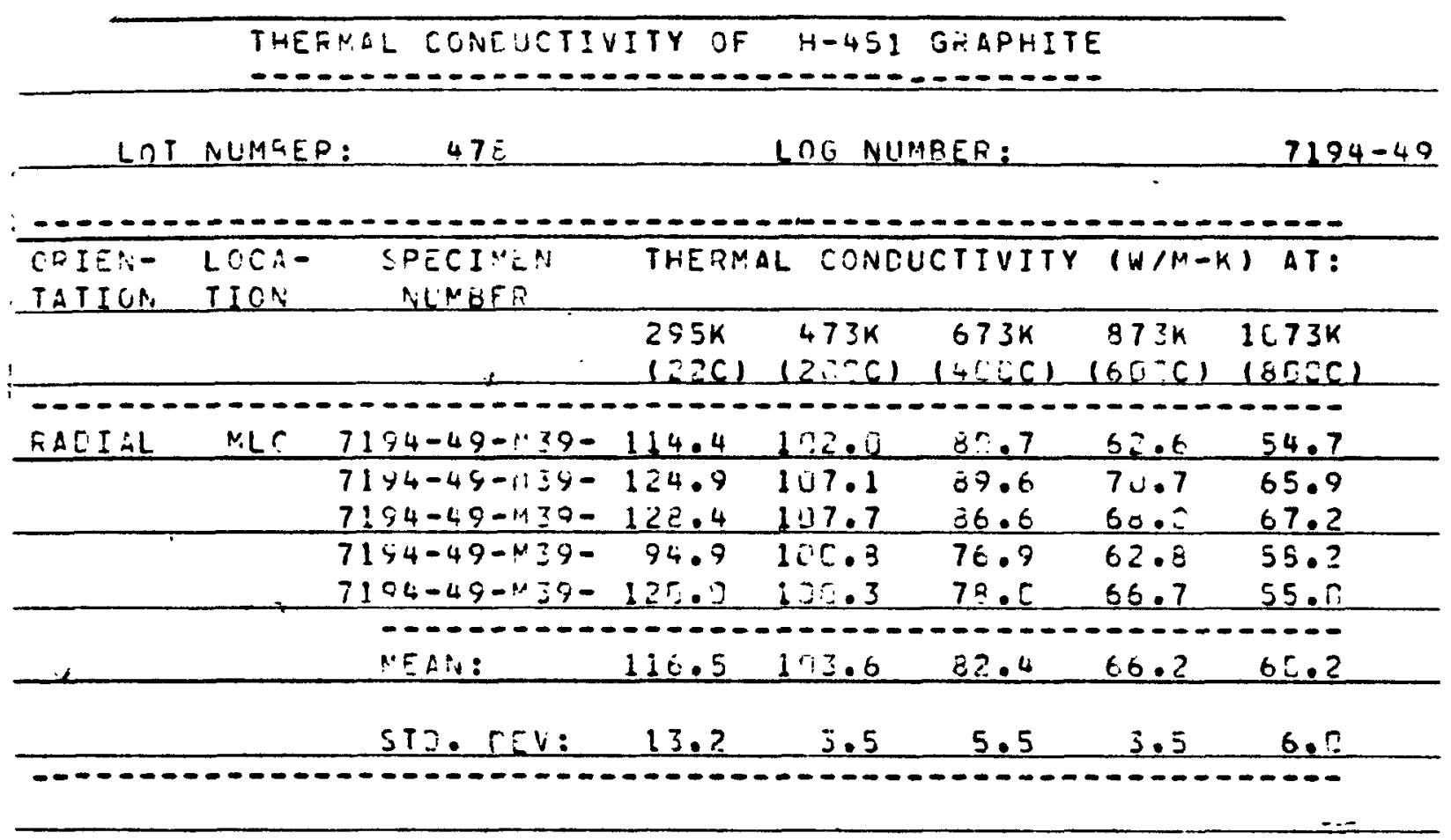


TABLE B-13

THLRTIAL CONOUTCTIVITY OF H-451 GEAPHITE
LOT NUMELK:
478
LOG NUMBER:
$7 f 94-52$

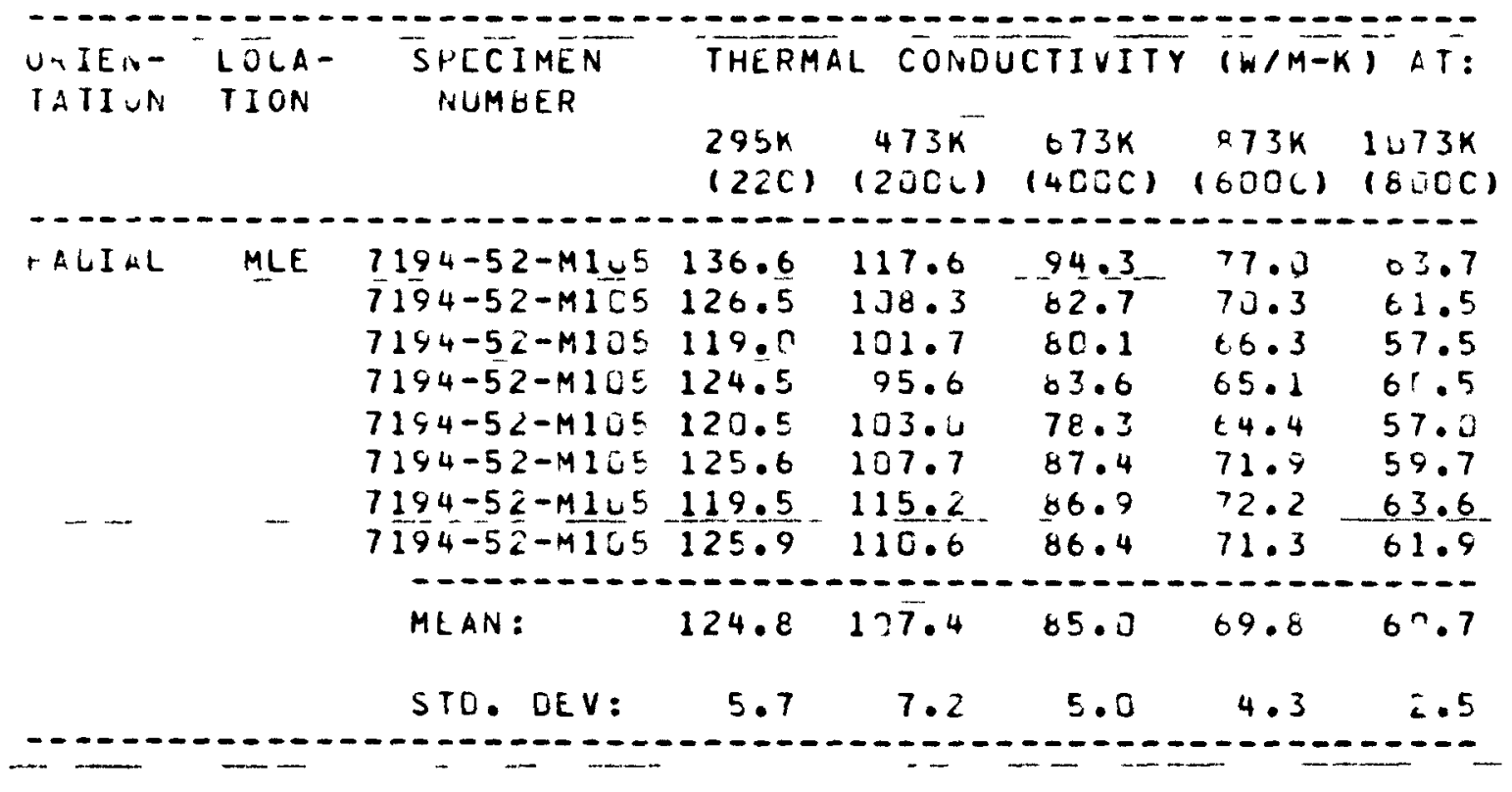


TABLE B-13 (Continued)

- THLRMAL CONDUCTIVITTY OF - H-4 TI GRAPHITE

LOT NUMBER: $478 \quad$ LOG NUMBER: 7f94-52

\begin{tabular}{|c|c|c|c|c|c|c|c|}
\hline \multirow[t]{2}{*}{$\begin{array}{l}\text { UHIE IN- } \\
\text { IAII IN }\end{array}$} & \multirow[t]{2}{*}{$\begin{array}{l}\text { LOCA- } \\
\text { II GN }\end{array}$} & \multirow[t]{2}{*}{$\begin{array}{c}\text { SPECIMEN } \\
\text { NUMECR }\end{array}$} & \multicolumn{3}{|c|}{ THERMAL } & $(n / M-K)$ & 1 AT: \\
\hline & & & $\begin{array}{l}295 k \\
(22 C)\end{array}$ & $\begin{array}{c}473 k \\
(2 \cup C C)\end{array}$ & $\begin{array}{c}673 K \\
(4 n O C)\end{array}$ & $\begin{array}{c}873 K \\
(600 C)\end{array}$ & $\begin{array}{l}1073 k \\
(8 C u C)\end{array}$ \\
\hline & $-\cdots$ & 20 & $-\infty-\infty$ & $-\cdots$ & $\cdots-\cdots$ & $\cdots \cdots$ & $\cdots$ \\
\hline$A X I A L$ & MLE & $\begin{array}{l}7194-52-M 98- \\
7194-52-M 98- \\
7194-52-M 98- \\
7194-52-M 98- \\
7194-52-M 98- \\
7194-52-M 98- \\
7194-52-M 98- \\
7194-52-M 98-\end{array}$ & $\begin{array}{l}122.3 \\
142.0 \\
134.2 \\
128.3 \\
131.1 \\
131.2 \\
137.3 \\
136.8\end{array}$ & $\begin{array}{l}115.4 \\
124.2 \\
121.7 \\
106.3 \\
120.1 \\
115.7 \\
118.3 \\
116.4\end{array}$ & $\begin{array}{r}89.7 \\
103.1 \\
92.7 \\
92.8 \\
94.2 \\
97.4 \\
98.7 \\
92.2\end{array}$ & $\begin{array}{l}75.6 \\
80.8 \\
70.5 \\
65.5 \\
77.5 \\
77.5 \\
79.2 \\
74.5\end{array}$ & $\begin{array}{l}67.3 \\
63.1 \\
57.6 \\
06.2 \\
67.4 \\
66.2 \\
69.5 \\
63.4\end{array}$ \\
\hline & & $m-m-m-n$ & $m-\infty-\infty$ & $\ldots$ & $=$ & 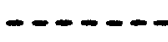 & - \\
\hline & & MEAN: & 132.9 & 117.3 & $95 \cdot 1$ & $75 \cdot 2$ & 65.0 \\
\hline & & STO. DEV: & 6.1 & 5.4 & $4 \cdot 3$ & 5.3 & 3.5 \\
\hline
\end{tabular}


TABLE B-T3 (Continued)

THE PMAL CONOUCTIVITY OF H-45I GFAPHITE
LOT NUMBLR:
478
LOG NUMBLR:
$1+94-52$

\begin{tabular}{|c|c|c|c|c|c|c|c|}
\hline \multirow[t]{2}{*}{$\begin{array}{l}\text { ORIE IN- } \\
\text { TAIIUN }\end{array}$} & \multirow[t]{2}{*}{$\begin{array}{l}\text { LOCA- } \\
\text { TIGN }\end{array}$} & \multirow[t]{2}{*}{$\begin{array}{l}\text { SPECIMEN } \\
\text { NUMEER }\end{array}$} & THERMAL & \multicolumn{2}{|c|}{ CONDUCTIVITY } & $(n / M-K)$ & J $A T:$ \\
\hline & & & $\begin{array}{l}295 K \\
(22 C)\end{array}$ & $\begin{array}{c}473 K \\
(2 J 0 C)\end{array}$ & $\begin{array}{l}673 K \\
(400 C)\end{array}$ & $\begin{array}{c}873 k \\
(603()\end{array}$ & $\begin{array}{l}1073 k \\
(8 \cap u C)\end{array}$ \\
\hline$\Delta L I A L$ & $M L C$ & $\begin{array}{l}7194-52-M 3 S- \\
7194-52-M 39- \\
7194-52-M 39- \\
7194-52-M 39- \\
7194-52-M 39- \\
7194-52-M 39- \\
7144-52-M 39- \\
7194-52-M 39-\end{array}$ & $\begin{array}{l}125 \cdot 3 \\
119.9 \\
104.5 \\
117.3 \\
128 \cdot 5 \\
123.1 \\
116 . C \\
122.1\end{array}$ & $\begin{array}{r}100.7 \\
98.1 \\
87.1 \\
100.2 \\
109.1 \\
103.0 \\
99.0 \\
110.6\end{array}$ & $\begin{array}{l}87.2 \\
00.1 \\
73.7 \\
81.1 \\
80.3 \\
82.7 \\
78.6 \\
85.6\end{array}$ & $\begin{array}{l}74.1 \\
66.7 \\
57.5 \\
69.4 \\
60.2 \\
69.0 \\
66.0 \\
72.2\end{array}$ & $\begin{array}{l}63.8 \\
58.6 \\
47.5 \\
62.5 \\
58.7 \\
61.9 \\
56.3 \\
59.3\end{array}$ \\
\hline & & MEAN: & 119.6 & 102.7 & 81.2 & 68.0 & 58.3 \\
\hline & & STO. OEV: & $7 \cdot 3$ & $\partial .5$ & 4.2 & 5.5 & 4.9 \\
\hline
\end{tabular}


TABLE B-13 (Continued)

THERMAL LONOUCTIVITY OF H-4ST GRAPHITE
LOI NUMBLR: 473 LOG INUMELR:

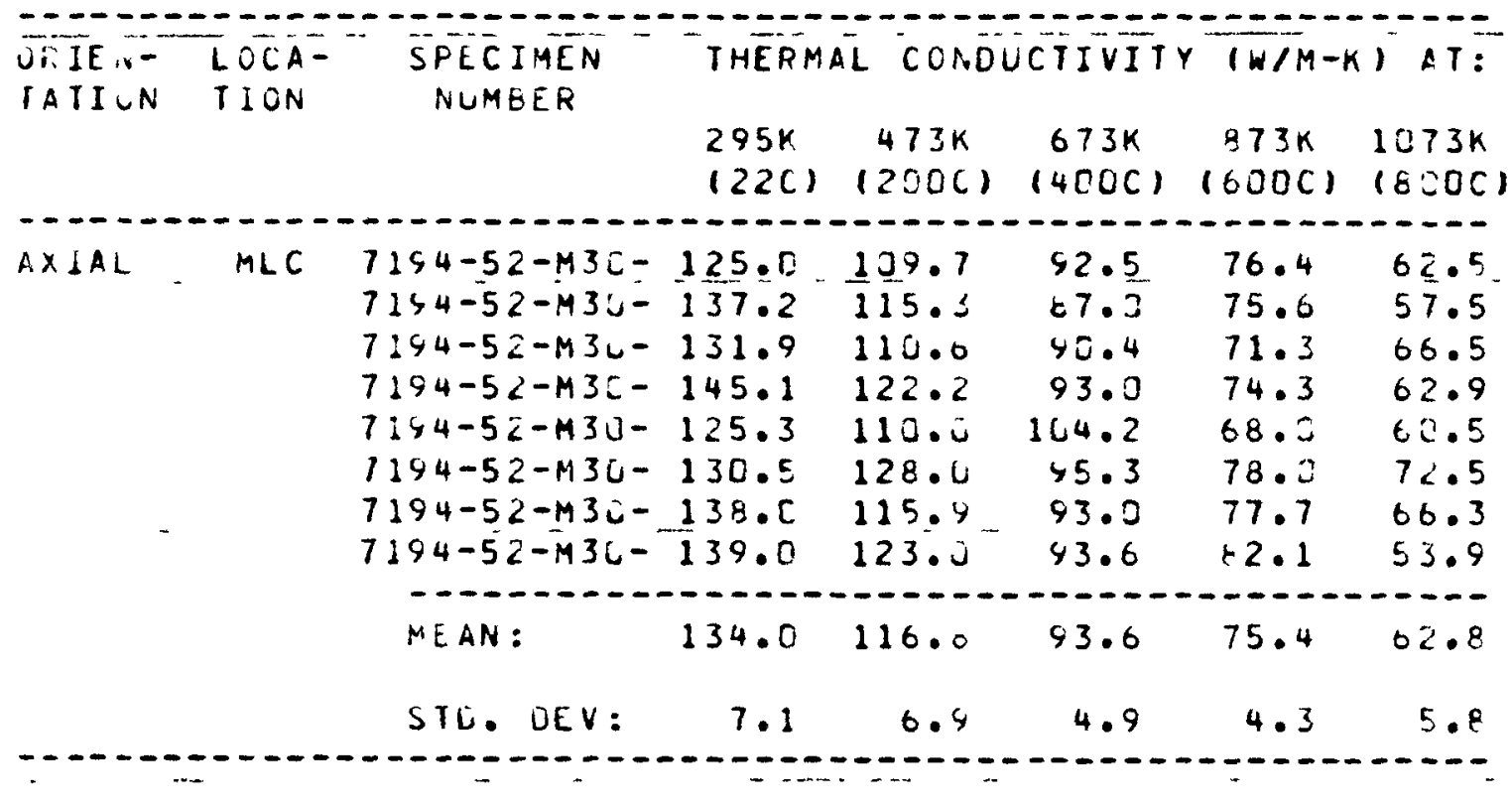


TABLE B-14

CHEMICAL PURITY

LOG 7194-49

SPECTROCHEMICAL BEPORT

GENERAL ATOMIC COMPANY

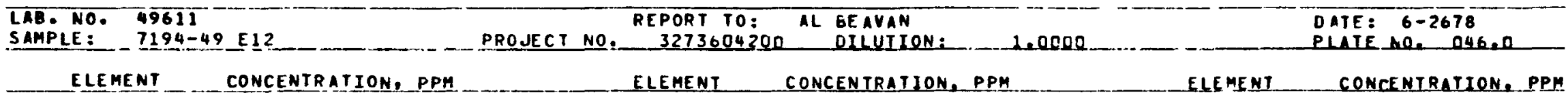

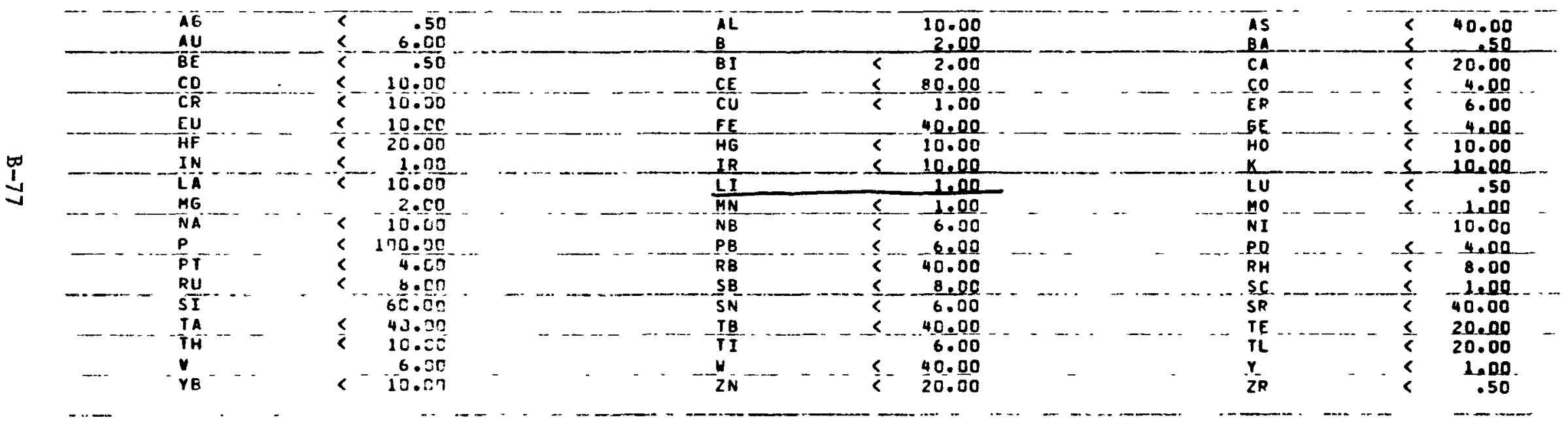

CONCENTRATION BASEO ON ORGINAL SAMPLE BEFORE DILUTION WITH DILUEN

TEANS GREATER THAN

$\checkmark$ MEANS LESS THAN THE SENSITIVITYCF THE SPECTROGRAPHIC PROCEDURE USED

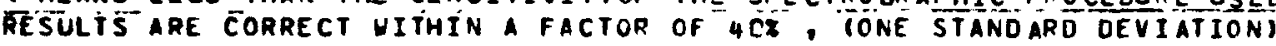




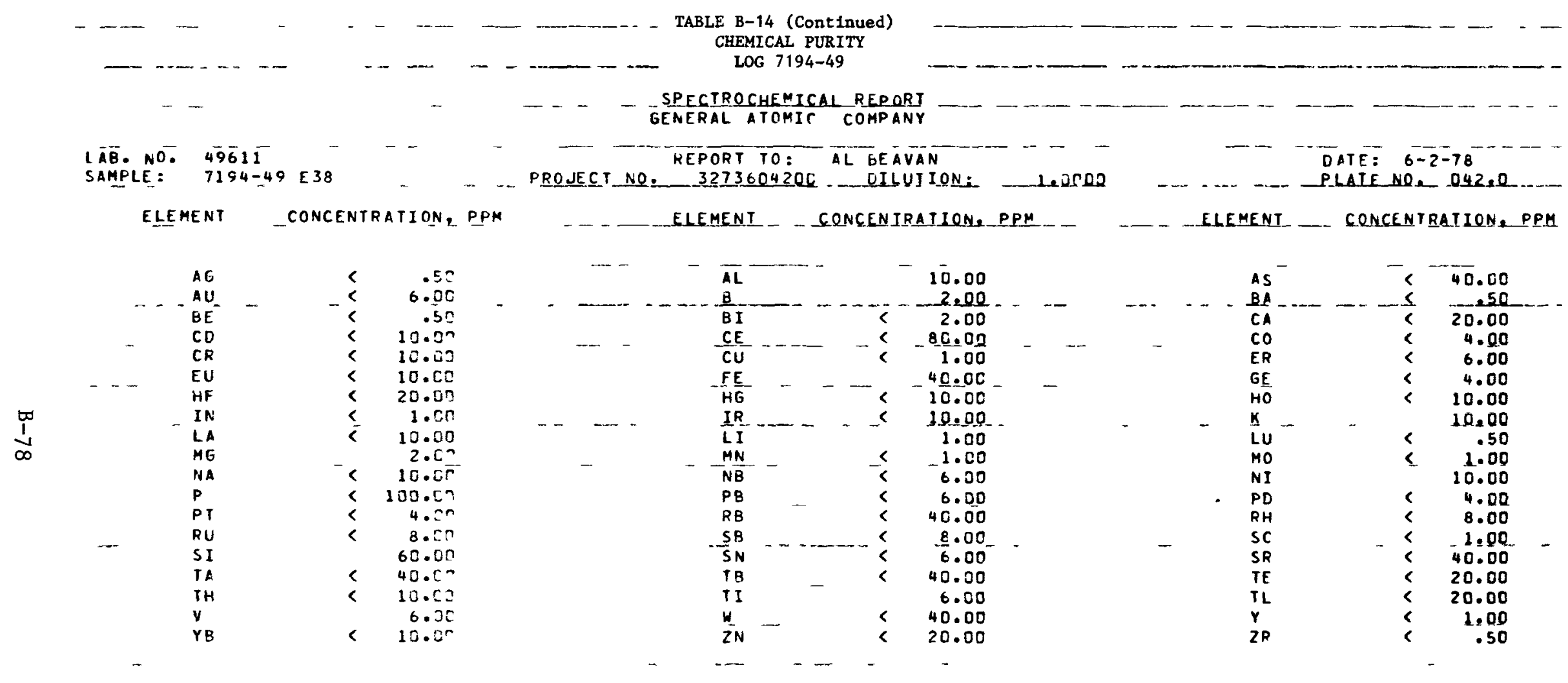

CONCENTRATION BASED ON OPGINAL SAMFLE BEFORE OILUTION WITH UILUENT $>$ MEANS GREATER THAN

\& MEANS LESS THAN THE SENSITIVITYOF THE SPECTROGRAPHIC PPOCEDURE USED RESULTS ARE CORRECT HITHIN A FACTLF OF 4 TZ, IONE STANDARD DFVIATIONI 
LAB. NO. 49611 SAMPLE: . 7194-49E60..

-..- TEPORT TO: TL GEAVAN

PROJECI NO. 3273604202 DEILUTION: D.0000 DATE: $6-2-78 \ldots$ ELEMENT CONCENTRATION, PPM _. CONCENTRATION, PPM
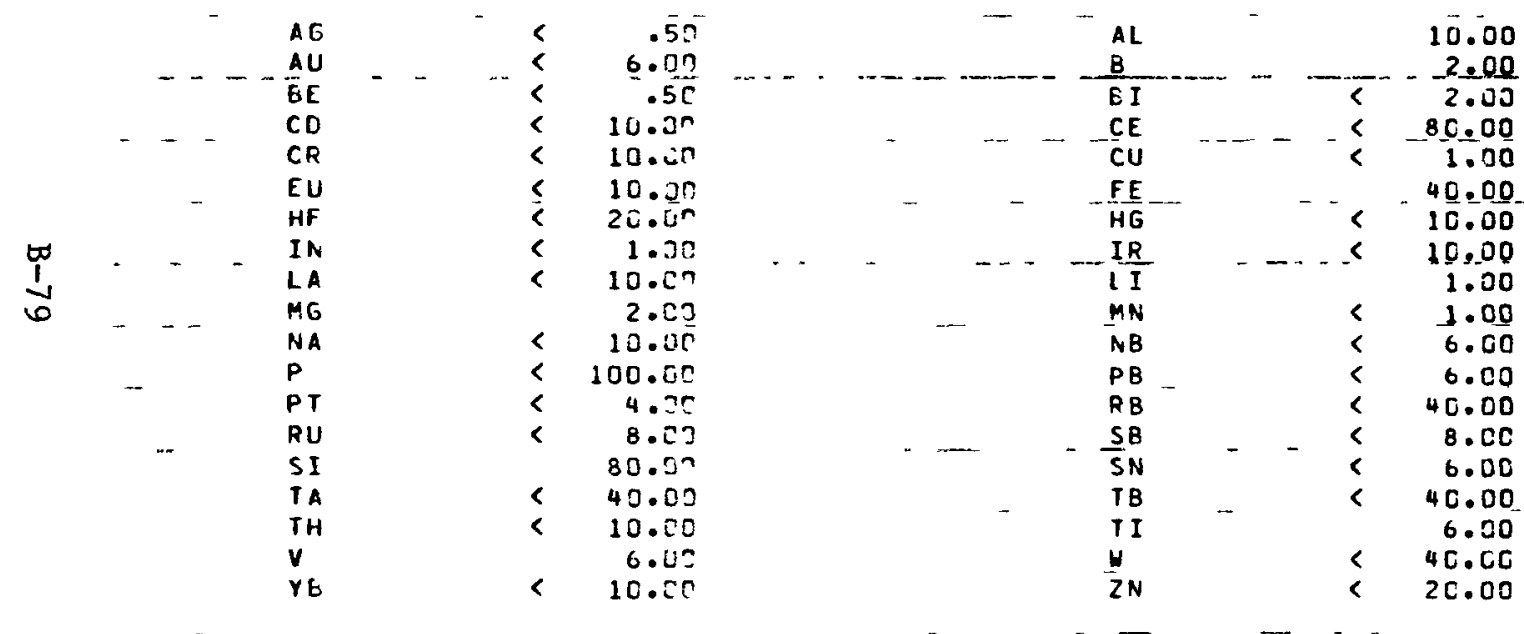

10.00
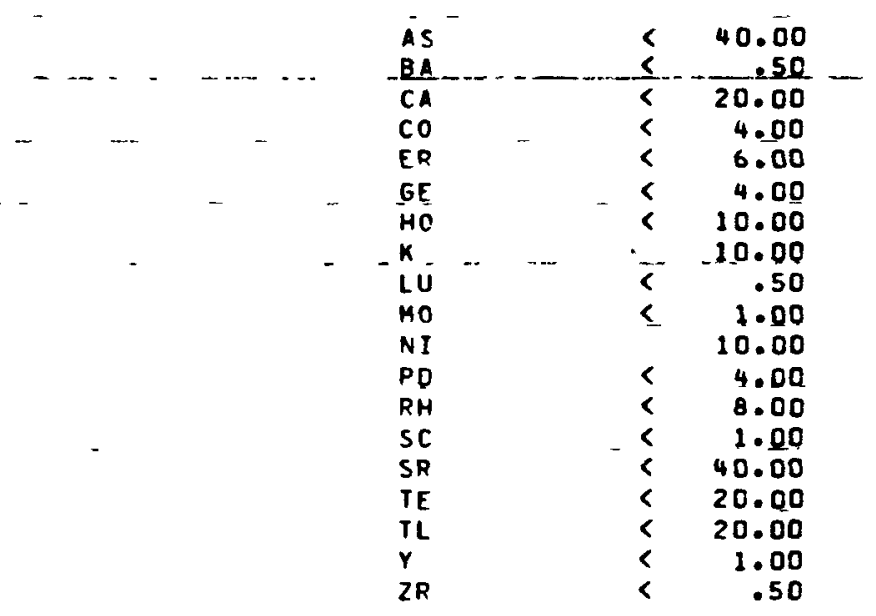

CONCENTRATION BASED ON ORGINAL SAMPLF BEFORE DILUTION WITH DILUENT

> MEANS GREATER THAN

< MEANS LESS THAN THE SENSITIVITYOF THE SPECTROGRAPHIC PROCEDURE USEN

RESULTS ARE CORRECT WITHIN A FACTOP CF $40 \dot{*}$, (ONE STAÑDARD DEVIATION) 
TABLE B-14 (Continued) CHEMICAL PURITY

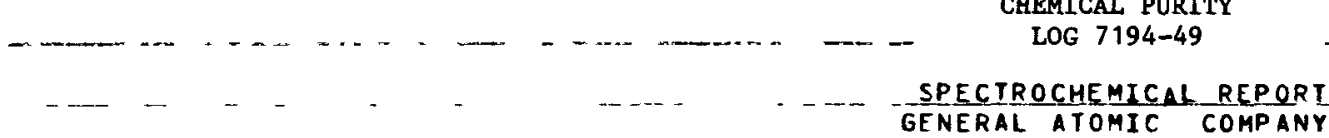

LAB. NOE. 49611 SAMPLE: 7194-49E90

REPORT TO: AL BEAVAN
PROJECT NOS 32736 C4200 - UILUTION: - 1.0000 ELEMENT - CONCENTRAIION. PPM -.
DATE: $6-2-78$ PLATE NO. 042.0
ELEMENT CONCENTRATION, PPM

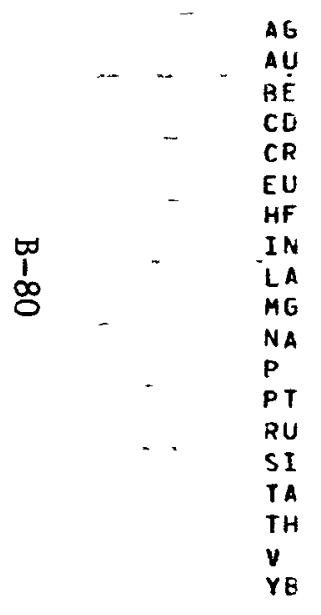

\begin{tabular}{|c|c|c|}
\hline & $<$ & $.5^{n}$ \\
\hline & $<$ & 6.0 \\
\hline & $<$ & .5 \\
\hline - & $<$ & $\begin{array}{l}10.00 \\
10.00\end{array}$ \\
\hline & $<$ & 10.00 \\
\hline & $<$ & 20.00 \\
\hline & $<$ & $1 . \subseteq ?$ \\
\hline & $<$ & $10.0 n$ \\
\hline & $<$ & $10 . \mathrm{cr}$ \\
\hline & $<$ & 130.00 \\
\hline & $<$ & 4.01 \\
\hline & $<$ & 8.03 \\
\hline & & 100.3 \\
\hline & $<$ & 43.50 \\
\hline & $<$ & 10.00 \\
\hline & $<$ & $\begin{array}{r}6.00 \\
13.00\end{array}$ \\
\hline
\end{tabular}

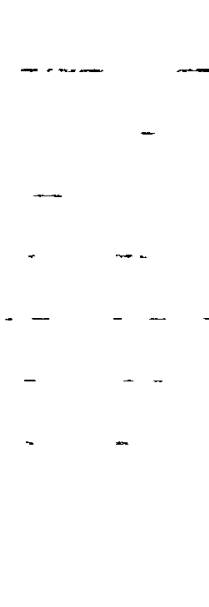

ELEMENT _ CONCENIRATION, PPM

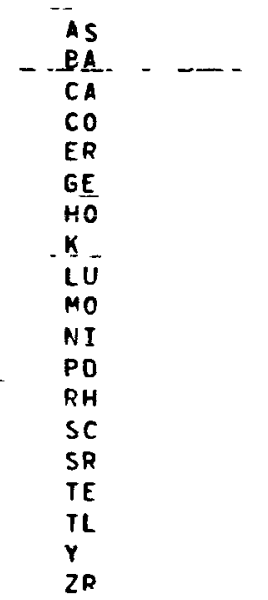

CONCENTRATION BASED ON ORGINAL SAMPLE BEFORE OILUTION WITH DILUENT 3 MEANS GREATER THAN

6 MEANS LESS THAN THE SENSITIVITYOF THE SPECTROGRAPHIC PROCEDURE USED RESULTS ARE CORRECT WITHIN A FACTOR OF 408 , (ONE STANDARD OEVIATION) 
TABLE B-14 (Continued)

CHEMICAL PURITY

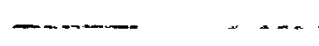

-... -

LOG $7194-49$

LAB. NO. $4 \overline{9} 611$

SAMPLE:- 7194-49M16

$-\cdots$

- SPECIROCHEMICAL REPORI

PROJECT NO. REPORT TO: AL GFAVAN

DATE: $6-2-78$

ELEMENT CONCENTRATION, PPM

_._ELEMENI - CONCENTPAIION, PPM _. . . _ ELEMENT _. CONCENIRATION, PPM

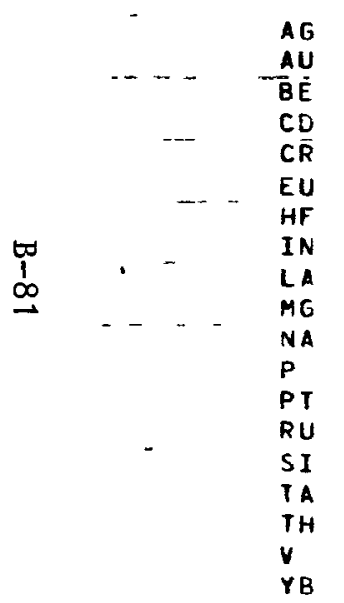

$<\quad .50$
$<\quad 6.00$
$<\quad 10.00$
$<\quad 10.00$
$<\quad 10.00$
$<\quad 20.00$
$<\quad 1.00$
$<\quad 20.00$
$<\quad 10.00$
$<\quad 40.00$
$<\quad 8.05$
$<\quad 80.05$
$<\quad 40.20$
$<\quad 6.00$
$<\quad 10.07$

$\cdots \cdots$

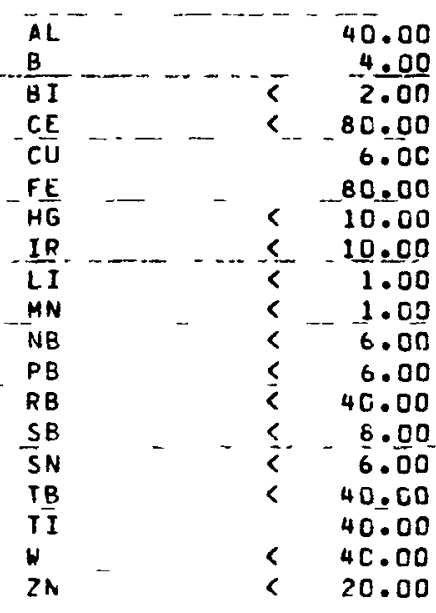

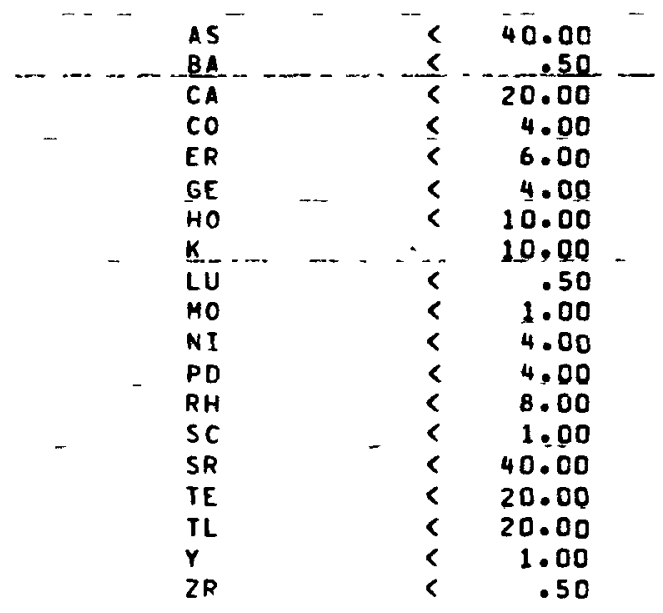

CONCENTRATION BASED ON OPGINAL SAMPLE BEFORE DILUTION WITH DILUENT

2 MEANS GREATFR THAN

< MEANS LESS THAN THE SENSITIVITYOF THE SPECTROGRAPHIC PROCEDURE USED

RESULTS ARE CORRECT WITHIN A FACTUR OF 4 CX, IONE STANDARD OEVIATIONI 
TABLE B-14 (Continued)

CHEMICAI PURITY
LOG $7194-49$

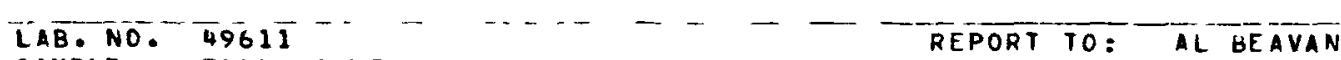

SAMPLE: - 7194-49 434

PROJECT NO: 3273604200 OILUTION: - 1.0000

DATE: 6-2-78

ELEMENT__ CONCENTRATION, PPM

ELEMENI -.. CONCENTRATIQNE PPM

ELEMENT

CONCENTRAIION, PPM
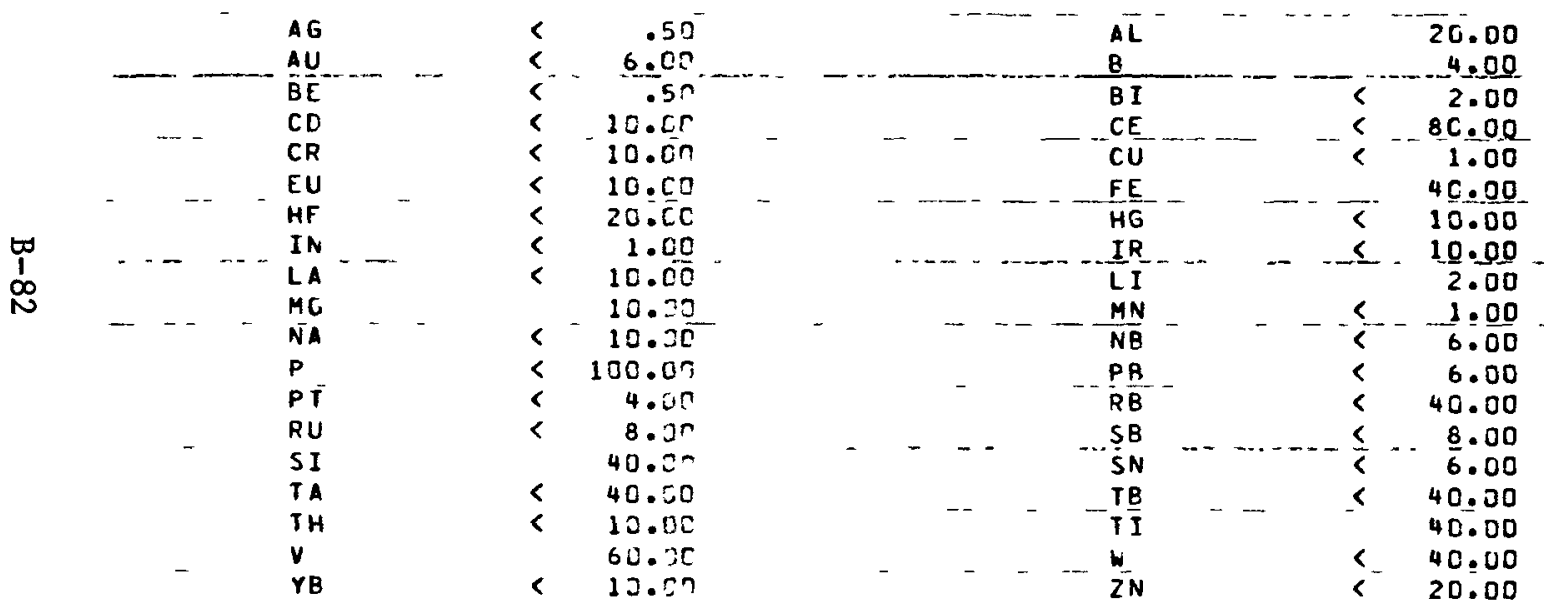

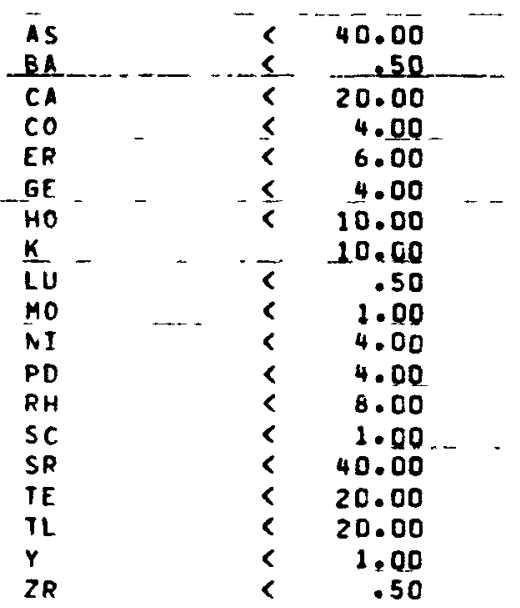

CONCENTRATION BASED ON OPGINAL SAMPLE BEFORE OILUTION WITH OILUENT MEANS GREATER THAN

C MEANS LESS THAN THE SENSITIVITYOF THE SPECTROGRAPHIC PROCEDURE USE

RESULTS ARE CORRECT WITHIN A FACTGR OF 4 ËZ, (ONE STANDARD DEVIATION) 
TABLE B-14 (Continued)

CHEMICAL PURITY
LOG 7194-49

LAB. $\overline{N O} \bar{O} \quad 4 \overline{9} 611$ SPECTROCHEMICAL REPQRI
ENERAL ATOMIC COMPANY

SAMPLE: $\quad 7194-49$ M60

REPORT TO: AL BEAVAN

HROJECT NO. 327360420 R L UILUIION:__. 1.0000

DATE: $\quad 6-2-78$

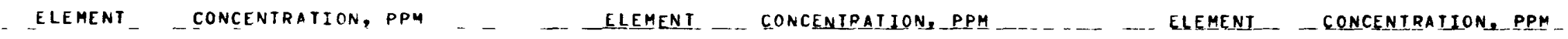

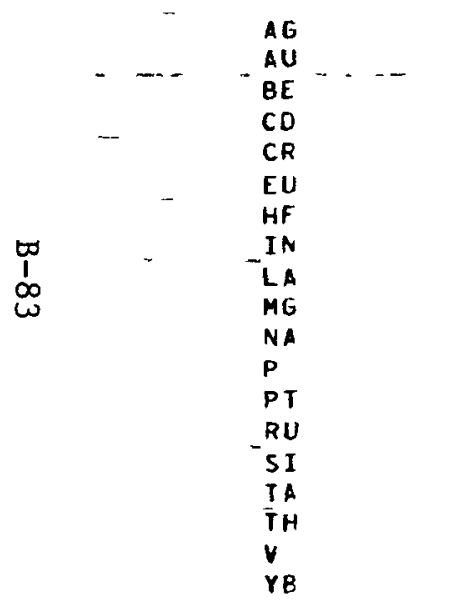

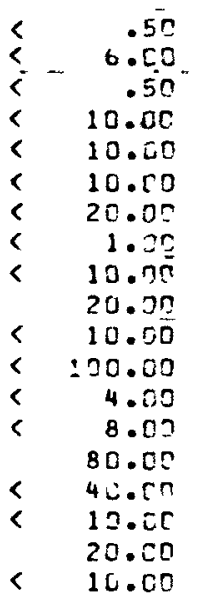

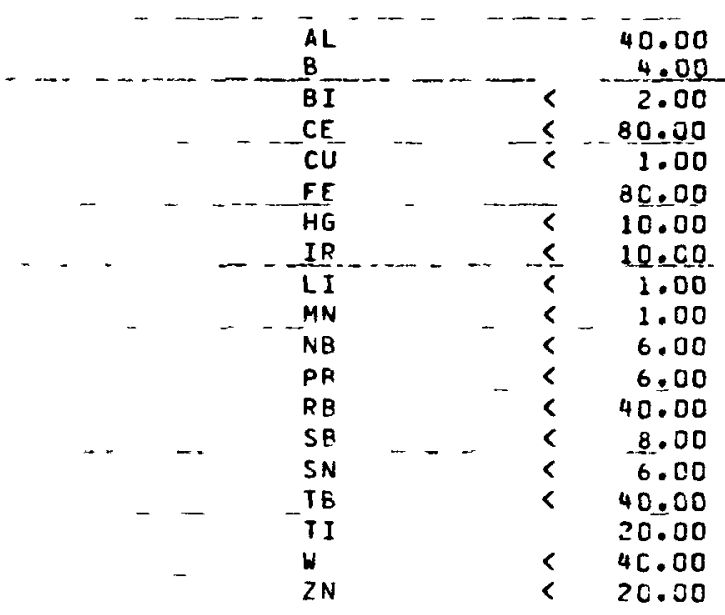

40.00
4.00
2.00
80.00
1.00
80.00
10.00
10.00
1.00
1.00
6.00
6.00
40.00
8.00
6.00
40.00
20.00
40.00
20.00

$\cdots \cdots$

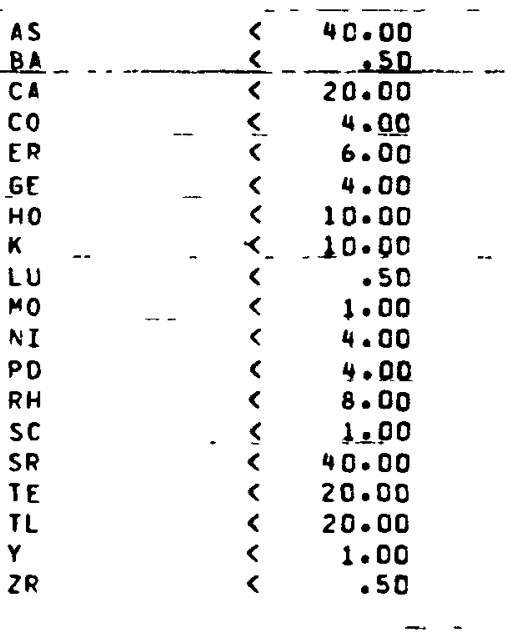

CONCENTRATION BASED ON OFGIIAAL SAMPLF BEFORE DILUTION WITH DILUENT

$>$ MEANS GREATER THAN

< MEANS LESS THAN THE SENSITIVITYOF THE SPECTROGRAPHIC PPOCEDURE USED

RESÜLTS ARE CORRECT HITHIN A FACTCP OF 4 CZ, IONE STANDARD DEVIATION) 

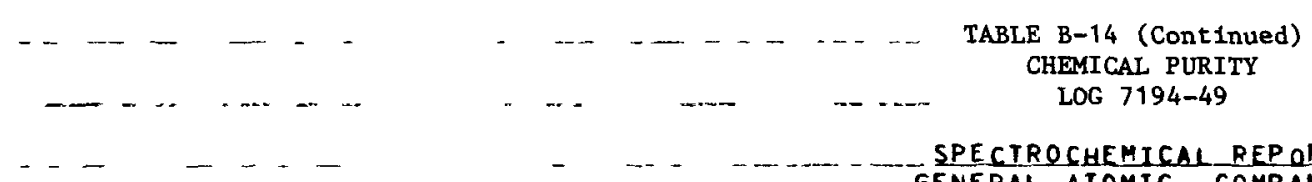
CHEMICAL PURITY

$-\cdots+\cdots$ - -1 SPECTROCHEMICAL REP ORT

LAB. NO. 49611 SAMPLE: $7194-49$ M9C .. PROJECT NO. REPORT TO: 3273604200 AL EEAVAN - - - - - -

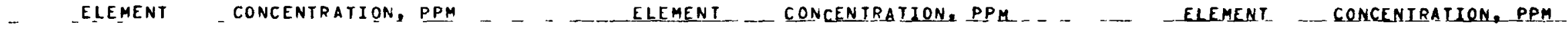
_ELEMENT _ CONCENTRATION, PPM _ _ _ ELEMENT _ CONGENTRAIION, PPM _ _ _ _ ELEMENT _. CONCENIRALION, PPM

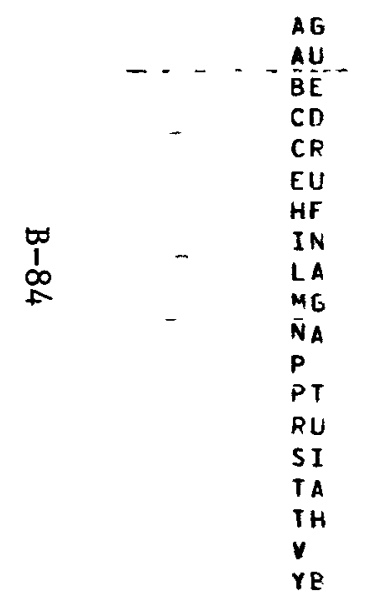

$\begin{array}{lr}< & .50 \\ < & 6.00 \\ < & 10.50 \\ < & 10.00 \\ < & 10.00 \\ < & 20.00 \\ < & 1.00 \\ <\quad 10.00 \\ <\quad 40.00 \\ <\quad 10.00 \\ <\quad 100.00 \\ <\quad 4.00 \\ & 8.00 \\ <\quad 40.00 \\ <\quad 10.00 \\ <\quad 10.00 \\ & 10.00\end{array}$

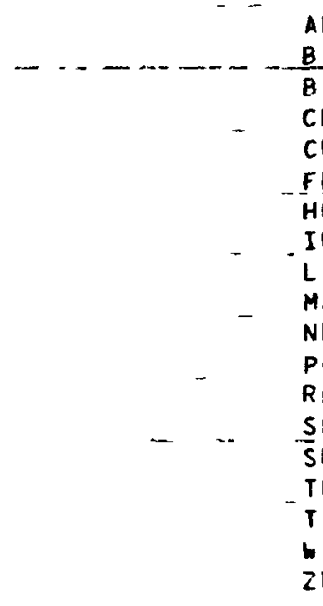

\begin{tabular}{|c|c|c|c|}
\hline $\begin{array}{l}\text { AS } \\
\text { BA }\end{array}$ & $\ldots$ & $<$ & 40.00 \\
\hline$C A$ & & $<$ & 20.00 \\
\hline CO & & $<$ & 4.00 \\
\hline$E R$ & & $<$ & 6.00 \\
\hline $\mathbf{G E}$ & & $<$ & 4.00 \\
\hline HO & & $<$ & 10.00 \\
\hline K & & & 10.00 \\
\hline LU & & $<$ & .50 \\
\hline MO & & $<$ & 1.00 \\
\hline NI & & $<$ & 4.00 \\
\hline$P D$ & & $<$ & 4.00 \\
\hline RH & & $<$ & 8.00 \\
\hline SQC & - & $\leq$ & 1.00 \\
\hline$S R$ & & $<$ & 40.00 \\
\hline TE & & $<$ & 20.00 \\
\hline $\mathrm{TL}$ & & $<$ & 20.00 \\
\hline $\mathbf{Y}$ & & $<$ & $1 . \mathrm{CO}$ \\
\hline $2 R$ & & & 8.00 \\
\hline
\end{tabular}

CONCENTRATION BASED ON ORGINAL SAMPLE BEFORE DILUTION WITH OILUENT $>$ MEANS GREATER THAN

S MEANS LESS THAN THE SENSITIVITYOF THE SPECTROGRAPHIC PROCEOURE USEO RESULTS ARE CORRECT WITHIN A FACTOF OF 4 CE, IONE STANDARD OEVIATIONI 
- $-\ldots \ldots \ldots$ TABLE B-15

LOG 7194-52

-

TAB. NO. 49611

PROJECT REPORT TO: AL EEAVAN

-_ ELEMENT _ CONCENTRATION, PPM _ _. _ ELEMENT _ CONCENTRATION, PPM _-_ _- ELEMENT

DATE: $6-2-78$

PLATE NQ. Q Q 22.0

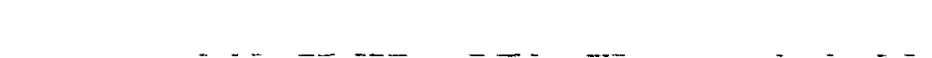

ELEENT CONCENTRAIION, PPM.

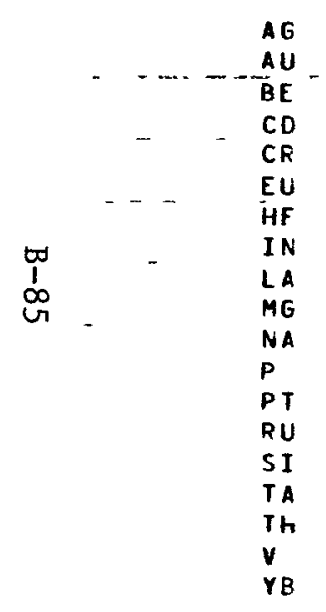

\begin{tabular}{|c|c|}
\hline & \\
\hline$<$ & $.5 \mathrm{C}$ \\
\hline$<$ & 6.07 \\
\hline$<$ & .50 \\
\hline$<$ & 10.00 \\
\hline$<$ & 10.00 \\
\hline$<$ & 10.00 \\
\hline$<$ & 20.00 \\
\hline$<$ & 1.00 \\
\hline$<$ & $10.5 \bar{c}$ \\
\hline & 10.50 \\
\hline$<$ & 10.00 \\
\hline$<$ & 100.00 \\
\hline$<$ & 4.00 \\
\hline$<$ & 8.70 \\
\hline & $40.0 \mathrm{C}$ \\
\hline$<$ & $40.5 n$ \\
\hline$<$ & 10.00 \\
\hline & 20.50 \\
\hline & ? \\
\hline
\end{tabular}

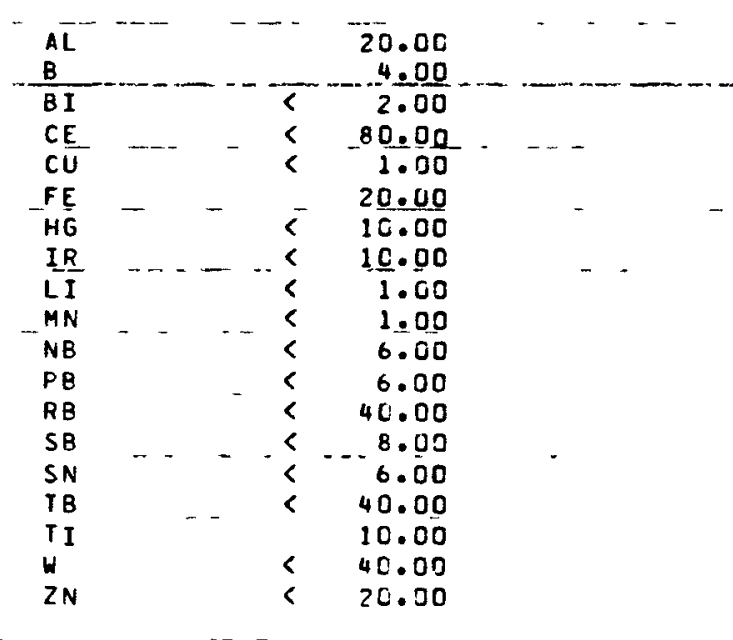

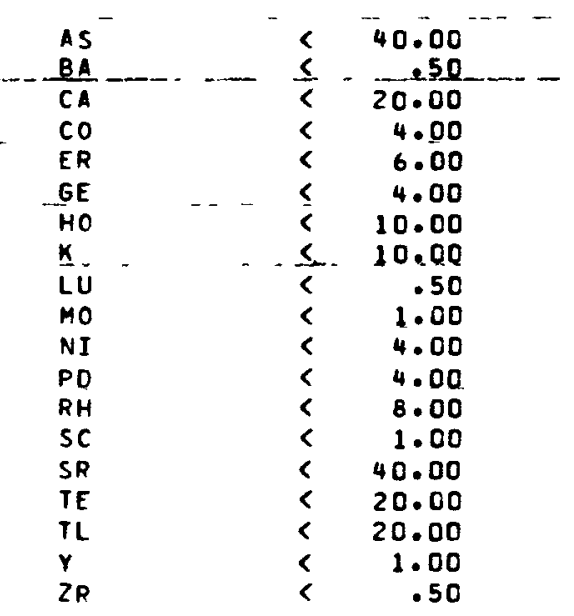

CONCENTRATION BASED ON ORGINAL SAMPLE BEFORE DILUTION WITH CILUENT

$>$ MEANS GREATER THAN

C MEANS LESS THAN THE SENSITIVITYLF THE SPECTROGRAPHIC PROCEOURE USEO

PESULLTS ARE CORRECT WITHIN A FACTOR OF 4 IZ, IONE STANDARD OFVIATIONI 
TABLE B-15 (Continued)

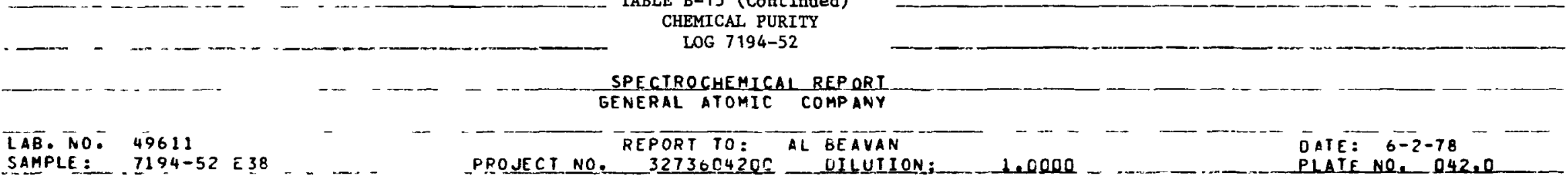

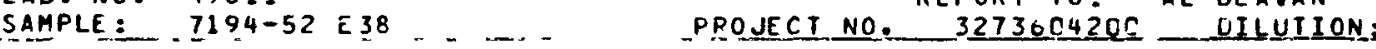

ELEMENT

CONCENTRATION, PPM

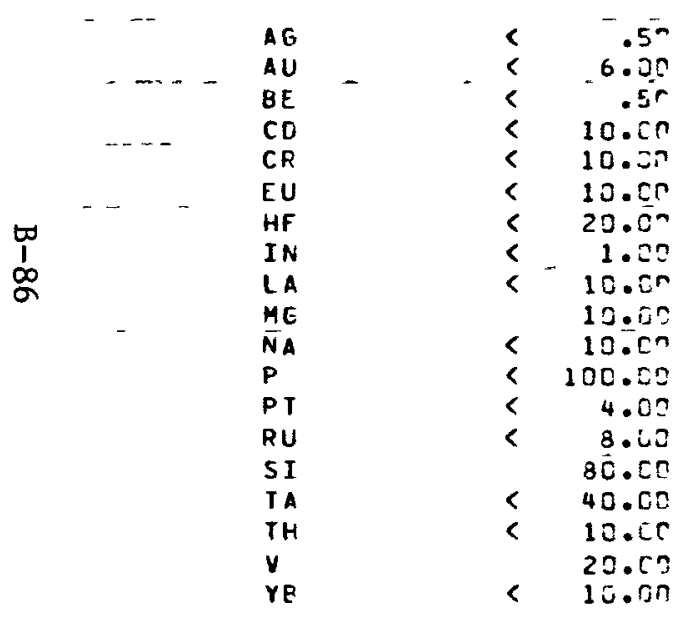

CONCENTRATION BASED ON OFGINAL SAMPLE BEFORE OILUTION WITH DILUENT

$>$ MEANS GREATER THAV

C MEANS LESS THAN THE SENSITIVIIYTOF THE SPECTROGRAPHIC PROCERURE USEN

RFSULTS ARE CORRECT WITHIN A FACTOR OF 4 CX, (ONE STANOARO DEVIATION) 


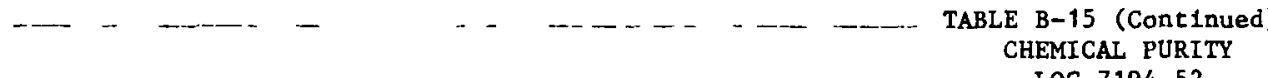

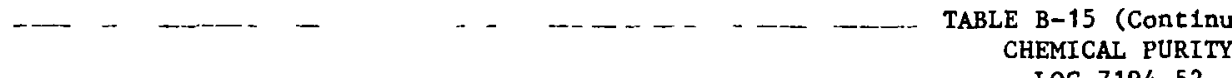

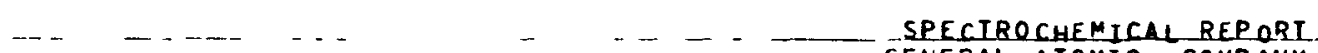

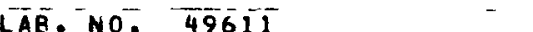

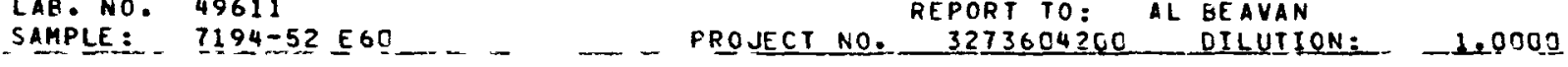

DATE: $6-2-78$

PLATE NQ. 042.0

- ELEMENT - _CONCENTRATION, PPY _ _ _ ELEMENT _ C CONCENIRAIION, PPM

ELE MENT

CONCENTRATION, PPH
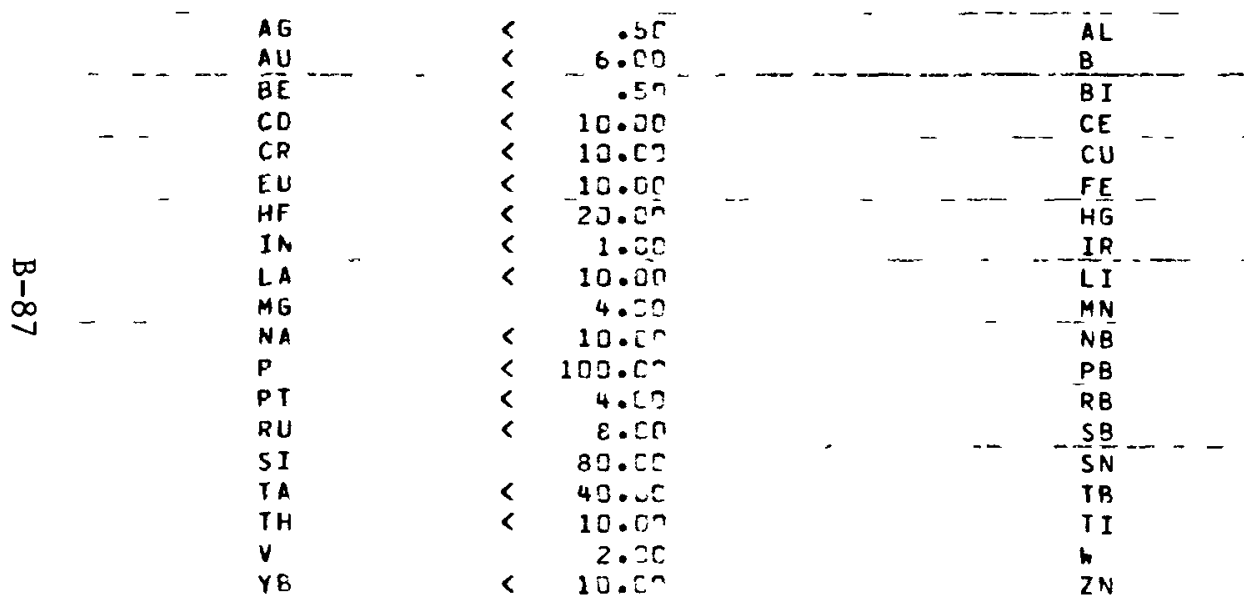

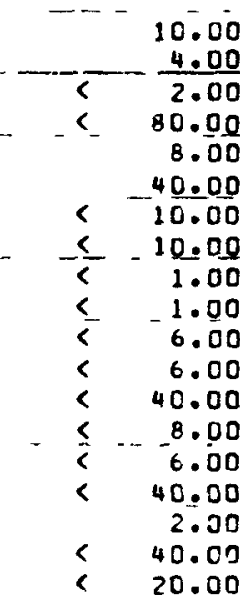

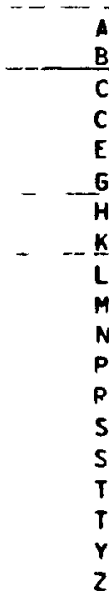

\begin{tabular}{|c|c|c|c|}
\hline $\begin{array}{l}\text { is } \\
\text { BA }\end{array}$ & & & $\begin{array}{r}40.00 \\
.50 \\
\end{array}$ \\
\hline$C A$ & & $<$ & 20.00 \\
\hline $\begin{array}{ll}\text { CO } \\
\text { CO }\end{array}$ & $-\quad-\ldots$ & $\leq$ & -4.00 \\
\hline $\begin{array}{l}\text { ER } \\
\text { GE }\end{array}$ & & < & $\begin{array}{l}6.00 \\
4.00\end{array}$ \\
\hline HO & & k & 10.00 \\
\hline$k$ & - & $\therefore$ & مئ0. \\
\hline LU & & $<$ & .50 \\
\hline MO & & $\leq$ & 1.00 \\
\hline N I & & $<$ & 4.00 \\
\hline PO & & $\leq$ & 4.00 \\
\hline PH & & $<$ & 8.00 \\
\hline SC & & $<$ & 1.00 \\
\hline SR & & $<$ & 40.00 \\
\hline TE & & $<$ & 20.00 \\
\hline TL & & $<$ & 20.00 \\
\hline Y & & 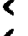 & 1.00 \\
\hline $2 \mathrm{R}$ & 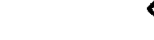 & 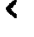 & .50 \\
\hline
\end{tabular}

CONCENTRATION BASED ON ORGINAL SAMPLF REFOPE OILUTION WITH DILUENT

2 MEANS GREATER THAN

C MEANS LESS THAN THE SENSIIIVITYOF THF SPECTROGRAPHIC PROCEOURE USEO

RESULTS ARE CORRECT WITHIN A FACTCR OF 4 SE, IONE STANDARO DEVIATIONI 
TABLE B-15 (Continued)

CHEMICAL PURITY

LOG 7194-52

SPECTROCHEMICAL REPORT

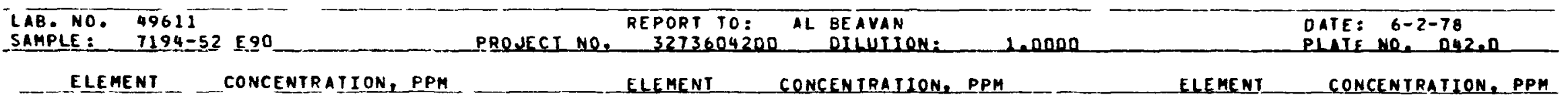

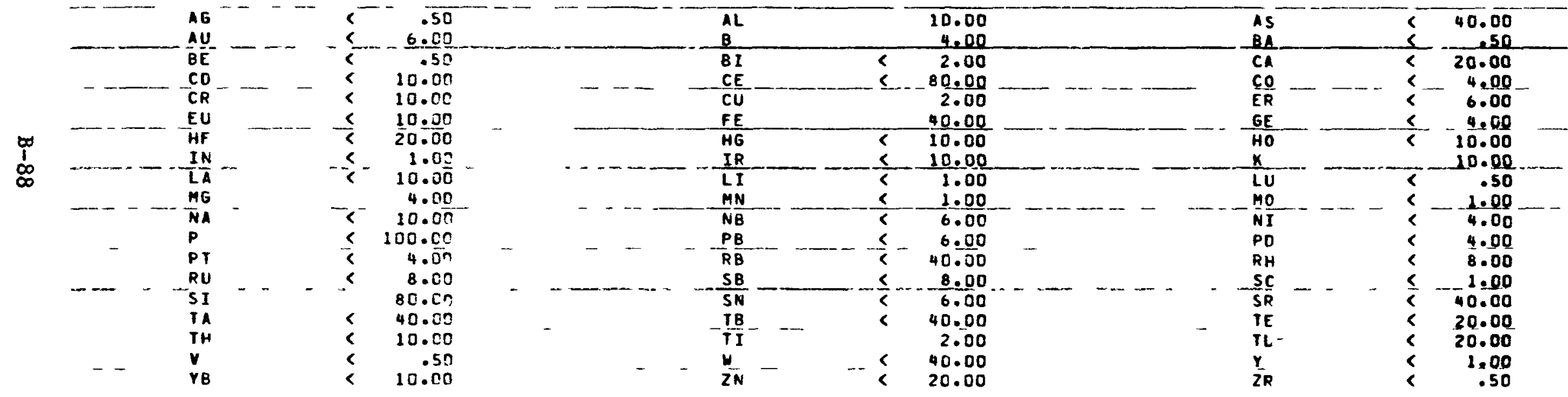

CONCENTRATION BASED ON ORGINAL SAMPLE BEFORE DILUT ION HITH DILUENT

S MEANS GREATER THAN

C MEANS LESS THAN THE SENSITIVITYOF THE SPECTROGRAPHIC PROCEDURE USED

RESULTS ARE CORRECT UITHIN A FACTOR OF 402 . (ONE STANDARD DEVIATION) 
TABLE B-15 (Continued)

CHEMICAL PURITY

LOG 7194-52

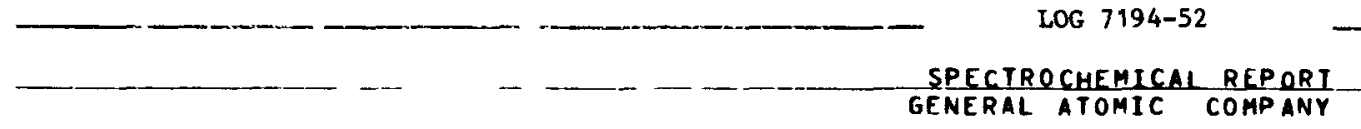

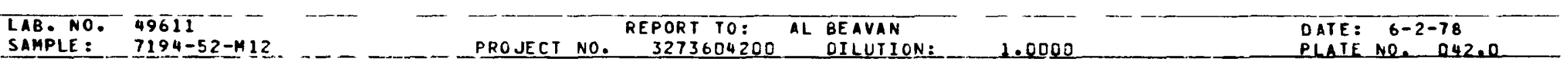

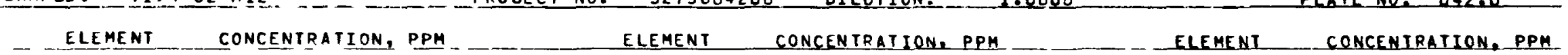

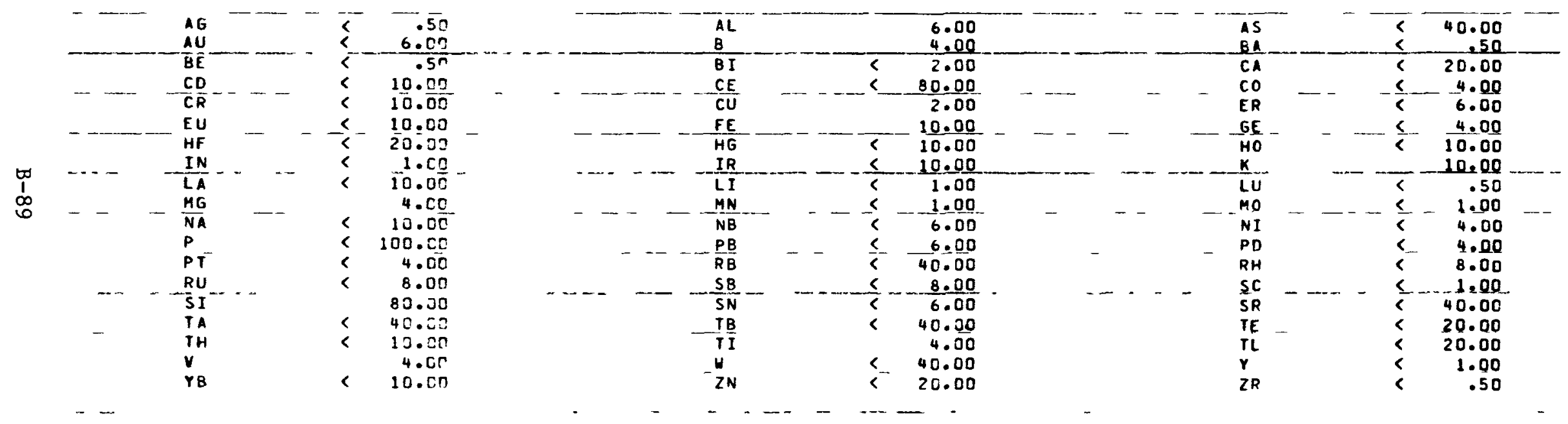

$-$

CONCENTKATION BASED ON ORGINAL SAMPLE BEFORE DILUTION HITH DILUENT

2 MEANS GREATER THAN

\ MEANS LESS THAN THE SENSITIVTTYOF THE SPECTROGRAPHTC PROCEDURE USEO

RESULTS ARE CORRECT WITHIN A FACTOD OF $40 \%$, IONE STANOARD DEVIATIONI 
TABLE B-15 (Continued)

CHEMICAL PURITY

\section{LOG 7194-52}

GEECTROCMEMICAL REPORT

$\overline{\mathrm{AB} \cdot} \overline{\mathrm{NO}} \cdot \overline{49 \overline{11}}$

SAMPLE: $7194: 52$ H 34

REPORT TO:

AL BEAVAN

DILUTLON:

DATE: $6-2-78$

ELEMENT _- CONCENTRAIION, PPM

ELEMENT CONCENIRAITON. PPH CONCENIRALION. PPM

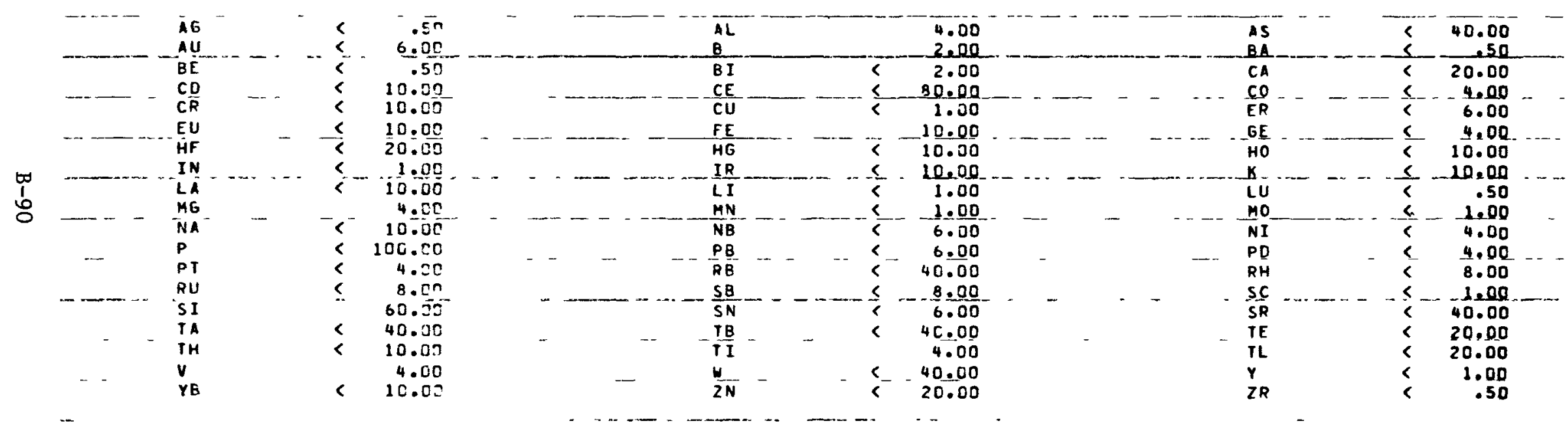

CONCENTRATION BASED ON ORGINAL SAMPLE BEFORE DILUTION WITH DILUENT MEANS GREATER THAN

C MEANS LESS THAN THE SENSITIVITYOF THE SPECTROGRAPHIC PROOCEDURE UNSED RESULTS ARE CORRECT WITHIN A FACTOR OF $40 \%$, (ONE STANDARD OEVIATION) 
TABLE B-15 (Continued)

CHEMICAI PURITY

LOG $7194-52$

SPECIROCHEMICAL REPORI
GENERAL ATOMIC COMPANY

\begin{tabular}{l} 
LAB. NO. 49611 \\
SAMPLE: $7194-52$ M6O REPORT TOE AL BEAVAN \\
\hline
\end{tabular}

DATE: $6-2-78$

PLATE NO. DU2,

ELEMENT CONCENIRATION, PPM ELEMENT CONCENTRATION. PPM

ELEMENI

CONCENIRAIION. PPA
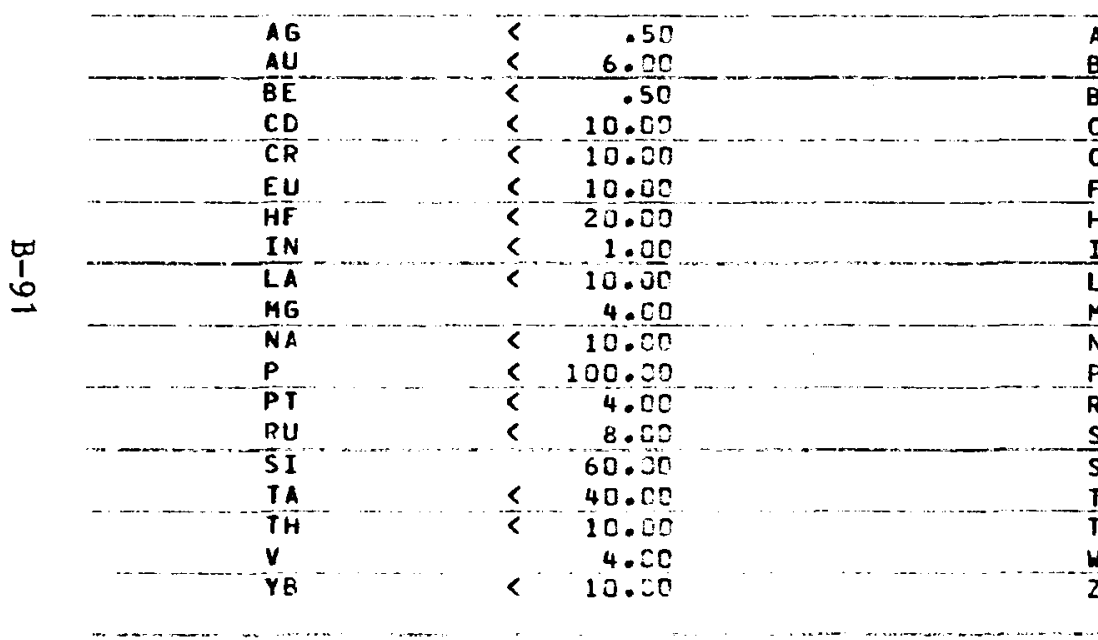

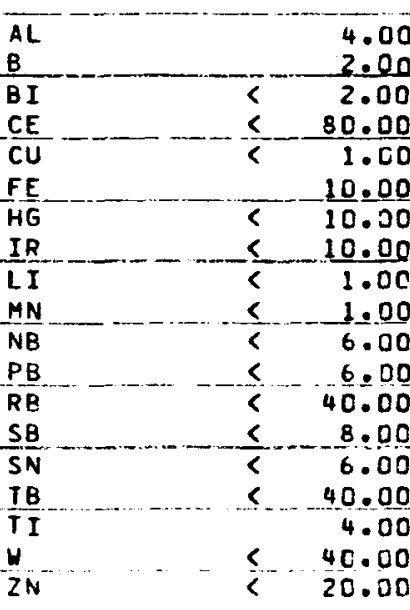

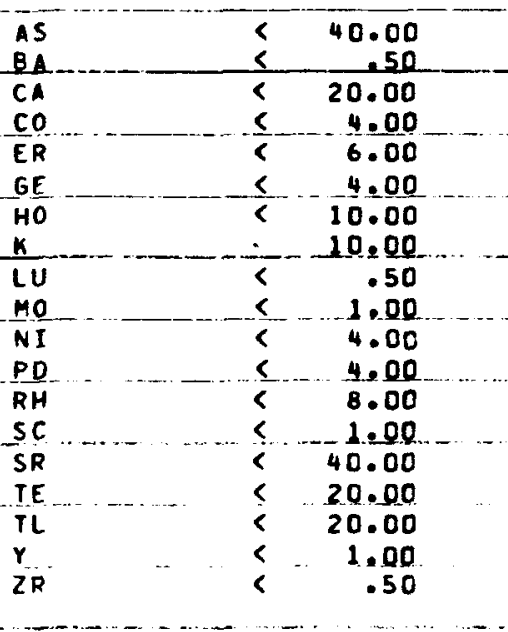

CONCENTRATION BASED ON ORGINAL SAMPLE BEFORE DILUTION WITH UILUENT

ᄀ MEANS GREATER THAN

C MEANS LESS THAN THE SENSITIVITYOF THE SPECTROGRAPHIC PROCEOURE USED

RESULTS ARE CORRECT WITHIN A FACTUR OF $40 X$, IONE STANOARO DEVIATIONI 
TABLE B-15 (Continued)

CHEMICAL PURITY

LOG $7194-52$

SPECTROCHEMICAL REPORT

GENERAL ATOMIC COMPANY

\begin{tabular}{l} 
LAB: NO. 49611 \\
SAMPLE: 7194-52 M9O REPORT TO: AL BEAVAN \\
\hline
\end{tabular}

ELEMENT - CONCENTRAIION, PPN

ELEMENT CONCENIRATION, PPM

ELEMENI

CONCENTRATION, PPM
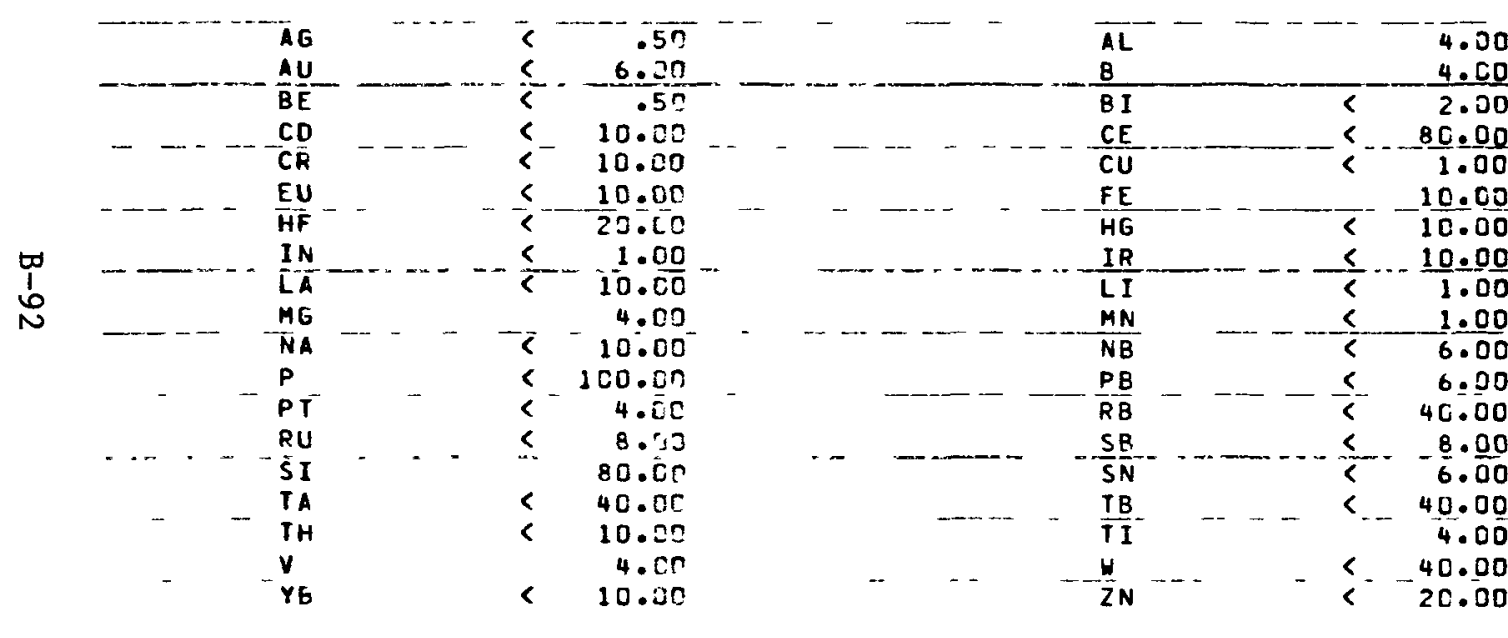

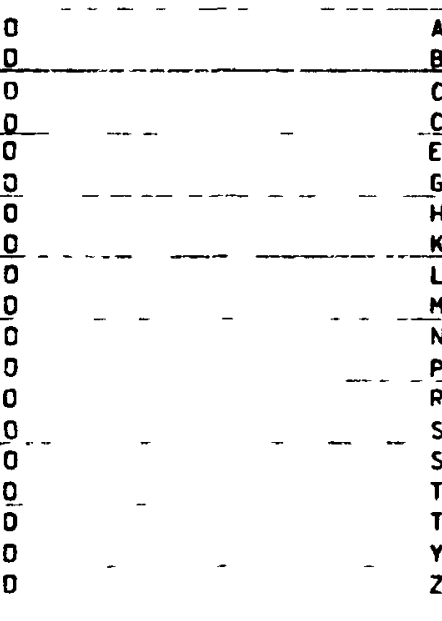

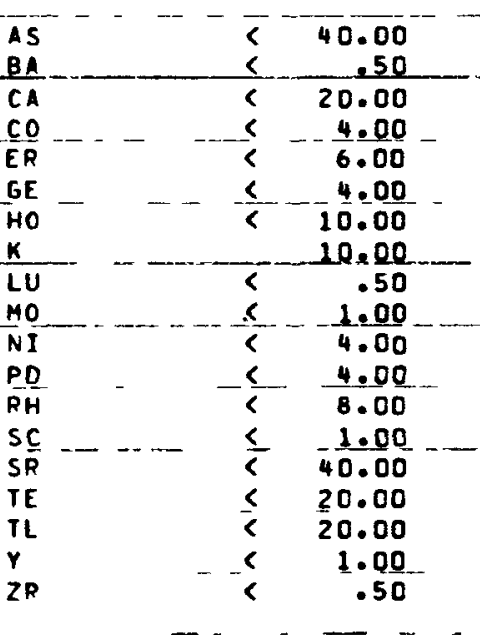

CONCENTRATION BASED ON ORGINAL SAMPLE BEFORE DILUTION HITH UILUENT

5 MEANS GREATER THAN

< MEANS LESS THAN THE SENSITIVITYOF THE SPECTROGRAPHIC PROCEDURE USEO

RESULTS ARE CORRECT WITHIN A FACTOR OF 4 OZ, (ONE STANDARD DEVIATION) 
TABLE B-16

CHEMICAL PURITY
LOG $7194-87$

SPECTROSHEMICAL REPORT

LAB. NO. 49611 REPORT TO: AL EEAVAN

3273604200

EILUIION

DATE: $6 \overline{6-2} \overline{-78}$

SAMPLE: $\quad 7194-87$ Ė12

ELEMEN

CONCENIRATIONE PPM

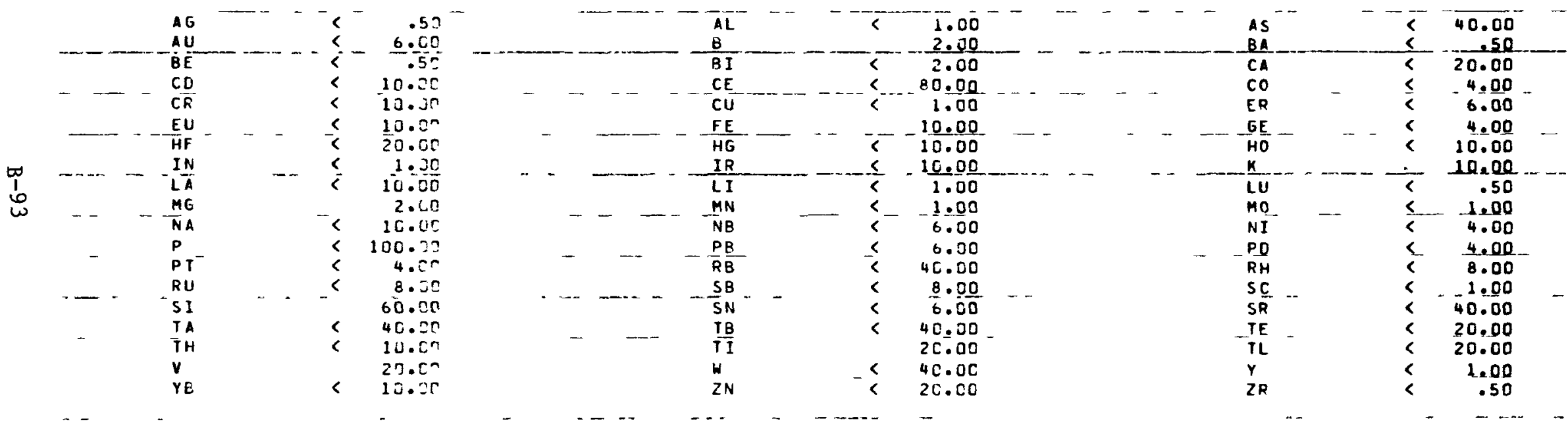

CONCENTRATION BASED ON ORGINAL SAMPLE BEFORE DILUTION WITH LILUENT

5 MEANS GREATER THAN

S MEANS LESS THAN THE SENSITIVITYCF THE SPECTROGRAPHIC PROCEDURE USEN

RESULTS ARE CORRECT WITHIN A FACTCP CF $40 Z$, (ONE STANDARO OEVIATION) 
TABLE B-16 (Continued)

CHRMICAL PURIT

LOG 7194-87

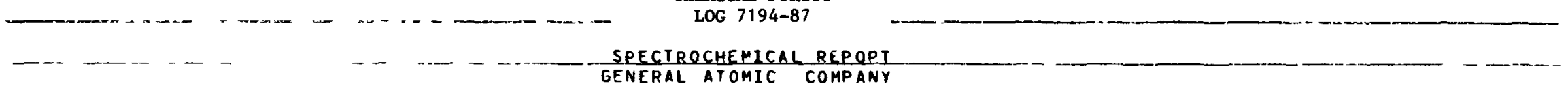

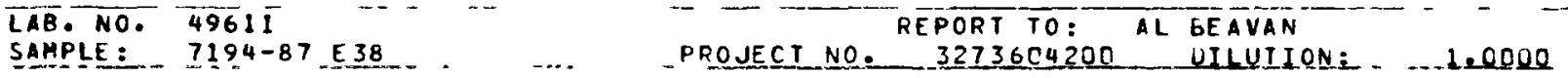

$\begin{array}{lll}\text { LAB. NO. } 4961 \overline{1} & \\ \text { SAHPLE: } & 7194-87 \text { E38 } & \text { REPORT TO: AL GEAVAN }\end{array}$

DATE: 6-2-78

DATE:

ELEMENT - CONCENTRATION, PPM _ _ _ ELEMENT CONCENIRATION PPM _ _ _ _ ELEMENT _ CONCENTRATION, PPM

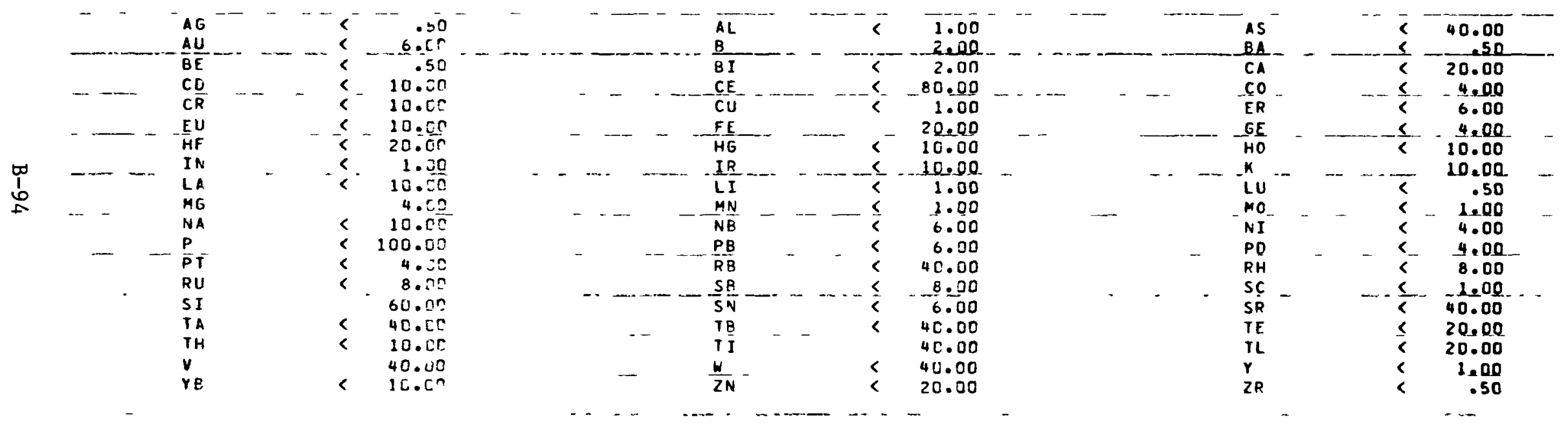

CONCENTRATION BASED ON ORGINAL SAMPLF BEFORE OILUTION WITH DILUENT

5 MEANS GREATER THAN

\ MEANS LESS THAN THE SENSITIVITYOF THE SPECTROGRAPHIC PROTERURE USEO

RESULTS ARE CORRECT WITHIN A FACTOR OF 4 EE, TONE STANOARE DEVIATIONI 
TABLE B-16 (Continued)

CHEMICAL PURITY

LOG 7194-87

SPECTROCHEMICAL REPORT

GENERAL ATOMIC COMPANY

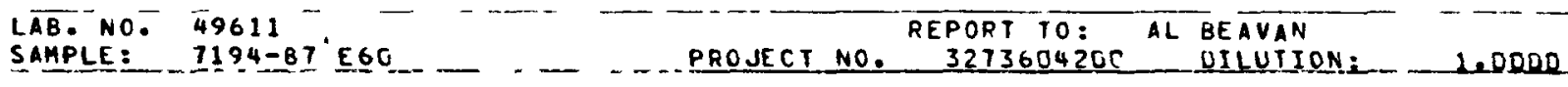

ELEMENT CONCENTRATION, PPM _ _._ELEMENT_COONCENIPATION, PPM -.

D.TE: $6-2-78$ PLAIE NO. 042 .

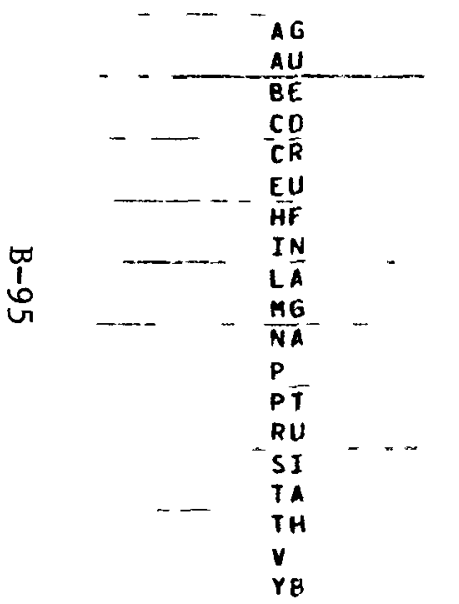

$\begin{array}{ll}< & -.50 \\ < & 6.00 \\ < & .50 \\ < & 10.00 \\ < & 10.00 \\ < & 10.00 \\ < & 20.00 \\ < & 1.00 \\ < & 10.00 \\ < & 2.00 \\ < & 10.07 \\ < & 100.07 \\ < & 4.05 \\ & 80.50 \\ < & 40.00 \\ < & 13.00 \\ < & 20.00 \\ < & 10.00\end{array}$
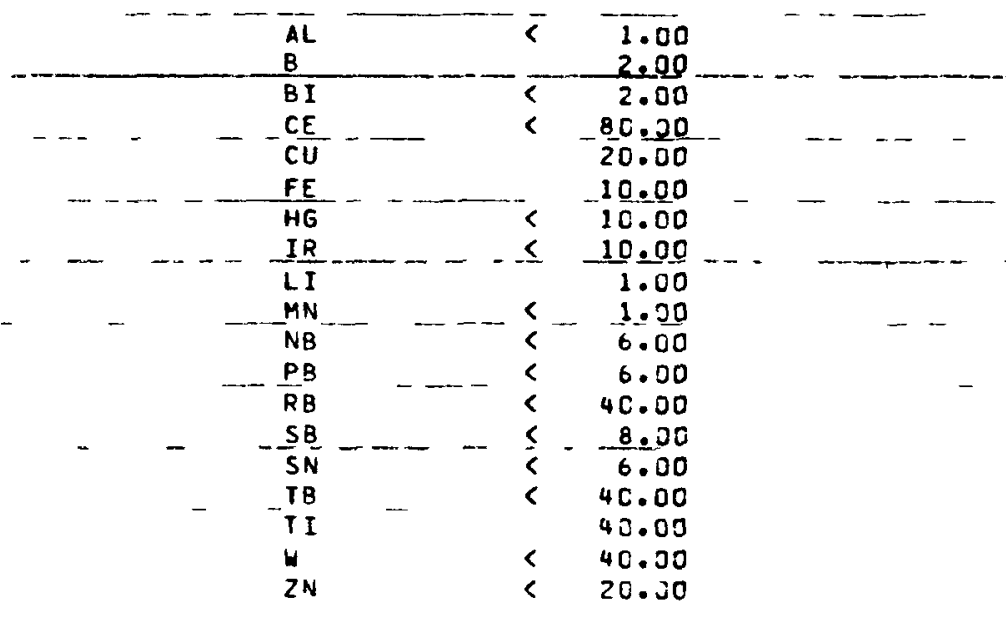

ELEMENT

CONCENIRAIION. PPM.

CONCENTRATION BASED ON ORGINAL SAMPLE BEFORE DILUTION WITH OILUENT

$>$ MEANS GREATER THAY

C MEANS LESS THAN THE SENSITIVITYOF THE SPECTROGRAPHIC PROCEDURE USET

RESULTS ARE CORRECT WITHIN A FACTOR OF 478 , (ONE STANDARO DEVIATION) 
TABLE B-16 (Continued)

CHEMICAL PURITY

LOG 7194-87 SPECTROCHEMICAL REPORI

$\mathrm{LAB} \cdot \mathrm{NO} \cdot 49 \overline{6} \overline{1}$

SAMPLE: 7194-87 E9

REPORT TO:

AL GE AVAN

PROJECT NO

3273604200 UILUTION.

DATE: $6-2-78$

ELEMENT CONCENIRATION, PPM

ELEMENT

CONCENTRATION, PPM
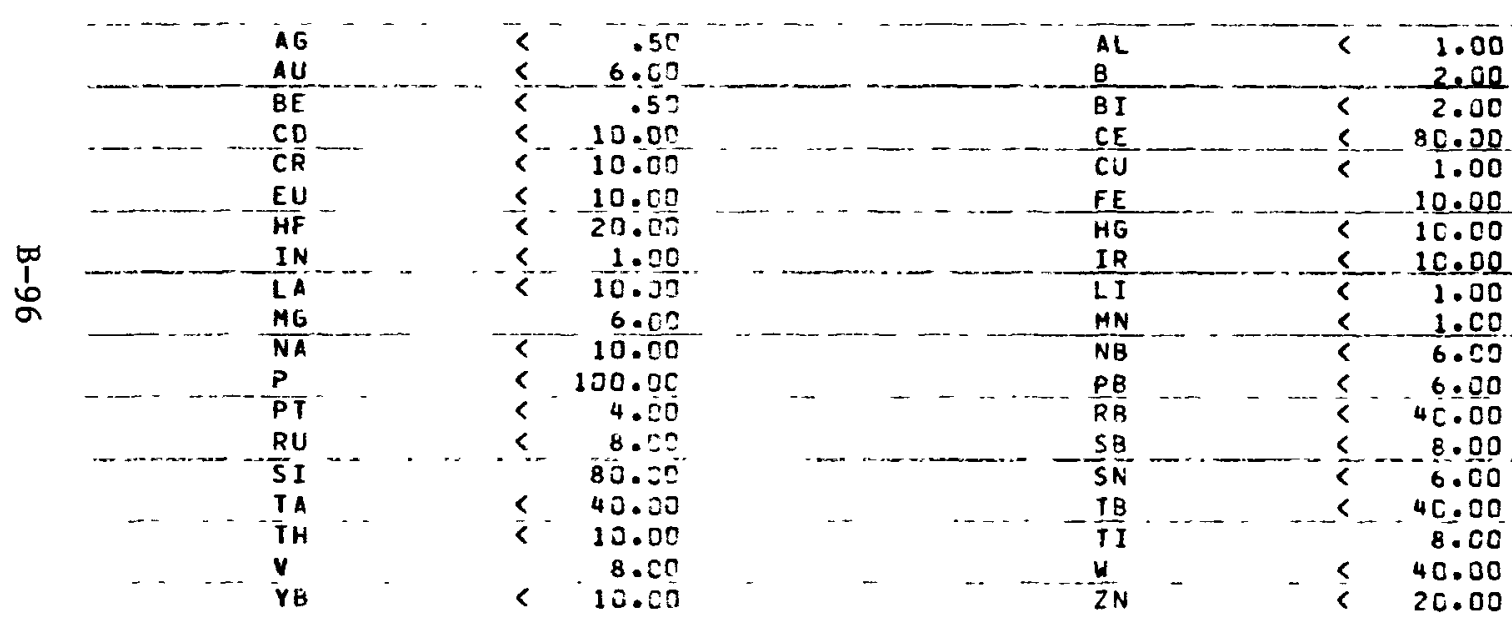

1.00
2.00
2.00
80.00
1.00
10.00
10.00
10.00
1.00
1.00
6.00
6.00
40.00
8.00
6.00
40.00
8.00
40.00
20.00

ב

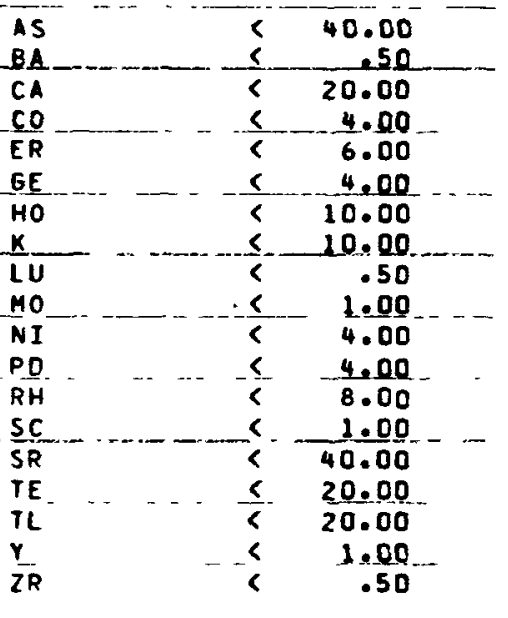

CONCENTRATION BASED ON OPGINAL SAMPLE BEFORE DILUTION WITH DILUENT

> MEANS GREATER THAN

S MEANS LESS THAN THE SENSITIVITYOF THE SPECTROGRAPHIC PROCEDURE USEO

RESULTS ARE CORRECT VITHIN A FACTOR OF 4 OX, IONE STANDARD DEVIATIONI 

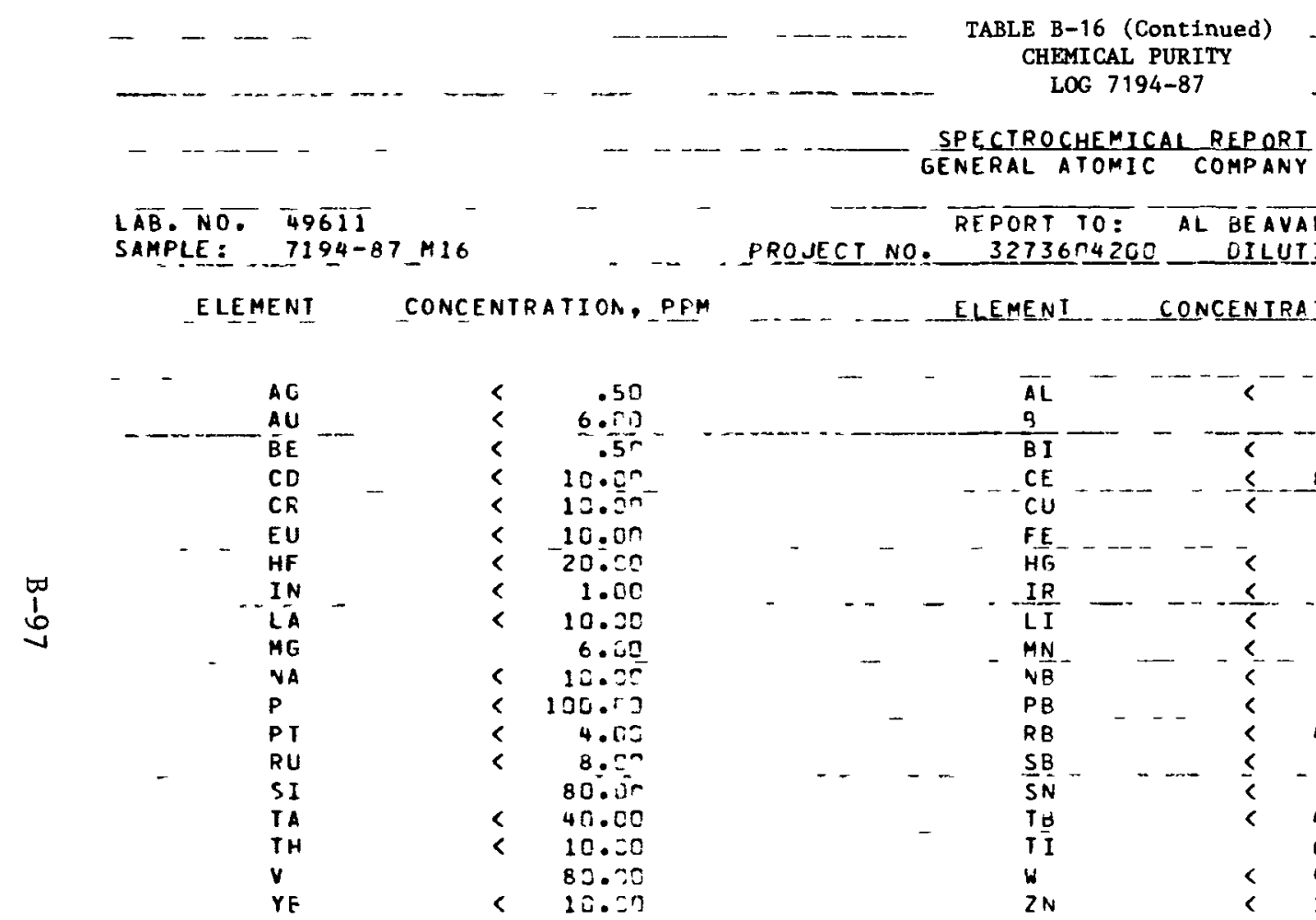

SPEECTROCHEMICAL REPORT

$\overline{A B} \cdot \bar{N} \overline{0 .} \overline{49611}$

7194-87_M16

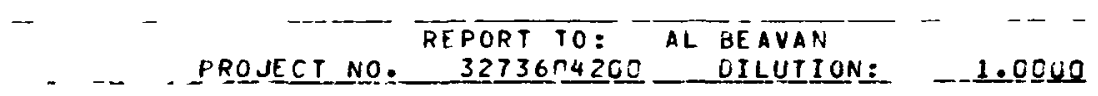

DATE: $6-2-78$ - DLATE NOE 042.0 ELEMENI- - CONCENTRATION, PPM

ELEMENT__- CONCENIRATION, PPM
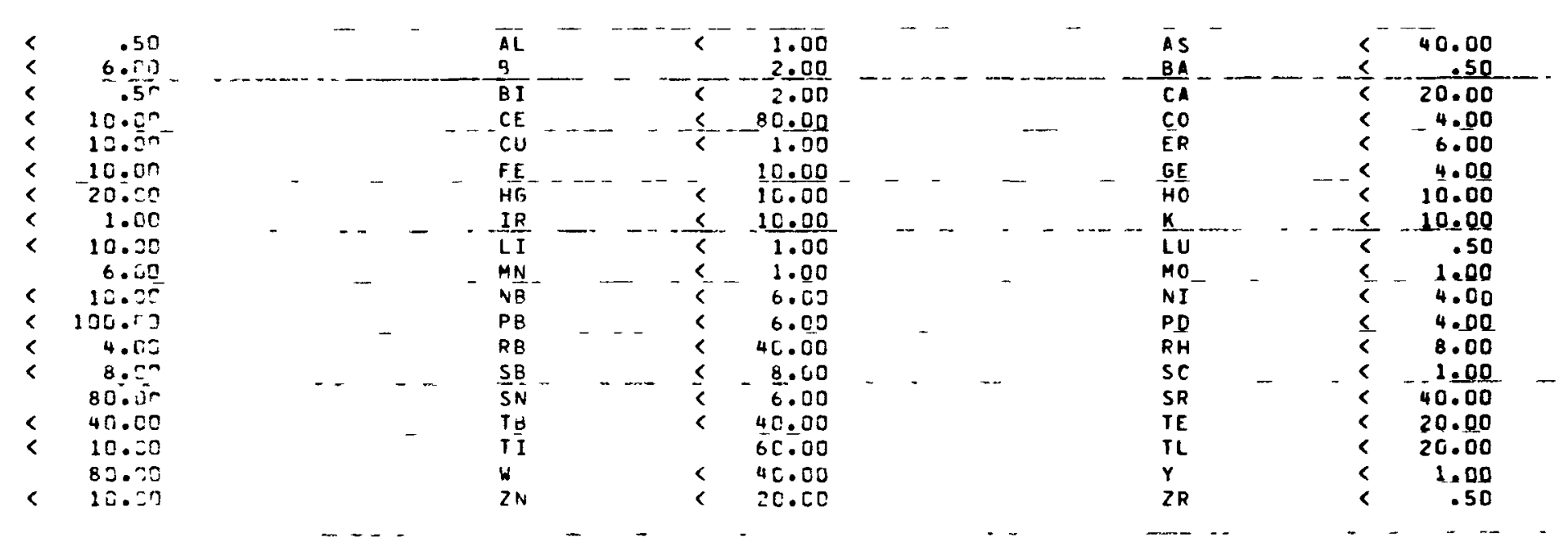

CONCENTRATION BASED ON ORGINAL SAMPLF BEFORE OILUTION AITH DILUENT > MEANS GREATER THAN

< MEANS LESS THAN THE SENSITIVITYCF THF SPECTROgRaphIC PROCE IURE USE

RESULTS ARE CORRECT WITHIN A FACTOF OF 4 IIX, (ONE STANDARD DEVIATION) 


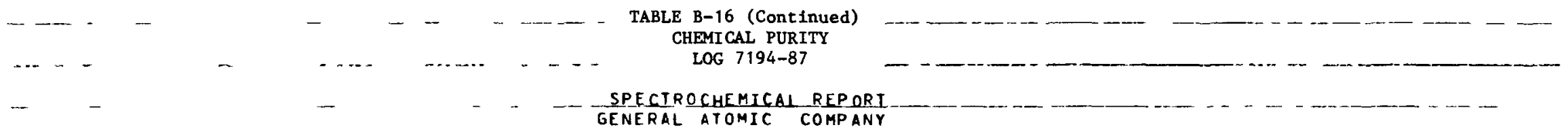

LAB. NO. 49611

SAMPLE: $\quad 7194-87 M 34 \quad \ldots$

REPORT TO: AL BEAVAN - - - - - DATE: 6-2-78

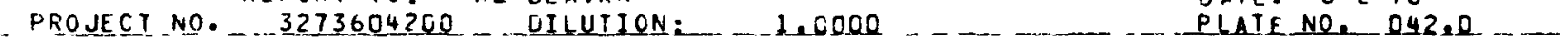

ELEMENT CONCENTPATION, PFM ELEMENI - CONCENIPAIION PPM _ . - ELEMENI - CONCENTRATIONE PPM

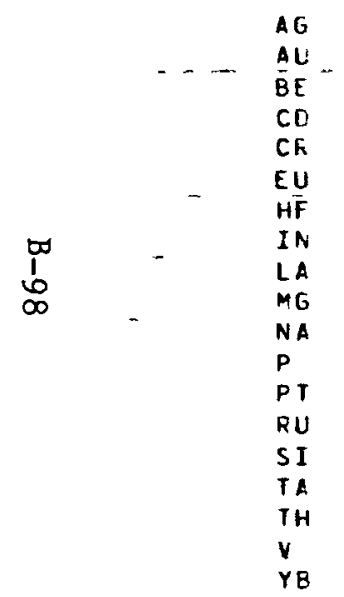

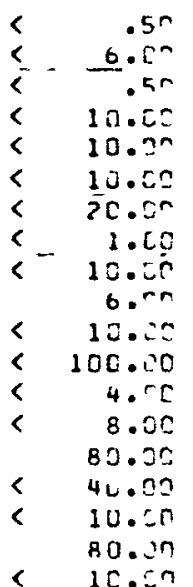

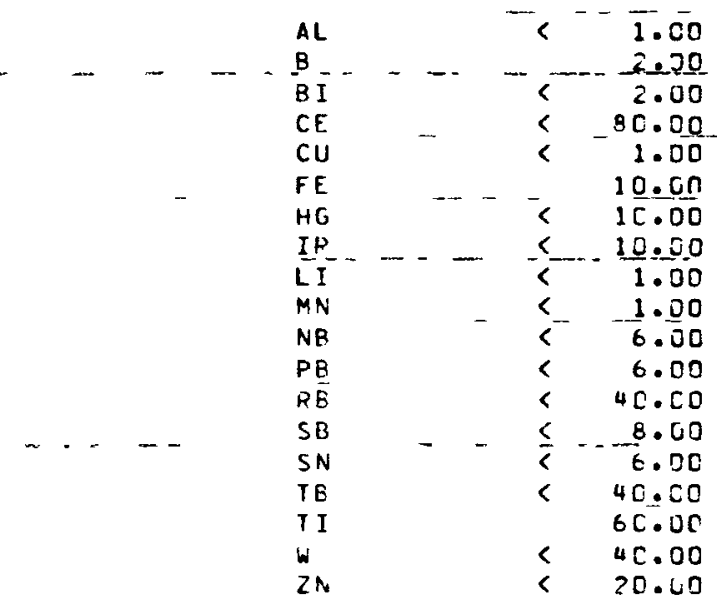

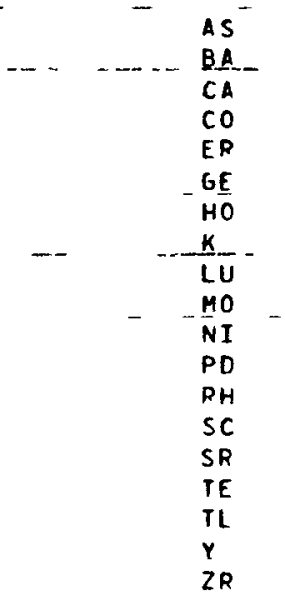

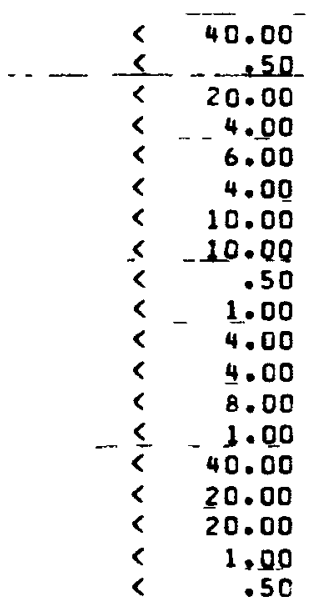

CONCENTRATION BASED ON OPEINAL SAMPLF PEFORE OILUTION WITH DILUENI

PEANS GREATER THAN

\ MEANS LESS THAN THE SENSITIVITYOF THE SPECTROGRAPHIC PROCLOURE USER

RESULTS ARE CORRECT WITHIN, FACTCR OF $4 \mathrm{CZ}$, (ONE STANDARD LEVIATION) 
i

TABLE B-16 (Continued)

CHEMICAL PURITY

$\ldots-\ldots \ldots . . .-1$

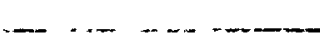

LOG 7194-87

$-\cdots+\cdots$

SPECTROCHEMICAL REPORT

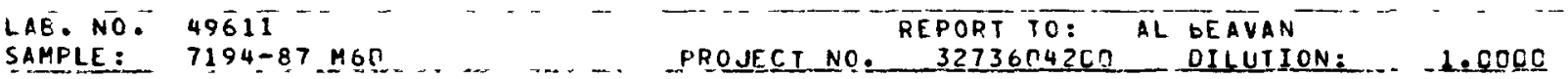

DATE: $6-2-78$

PLATE NO, 042 .

ELEMENT_ CONCEENTRAIION _ FPM _... _ _... ELEMENI _- CONCENIRATIONE PPM _. ELEMENT - CONCENTRATION, PPM

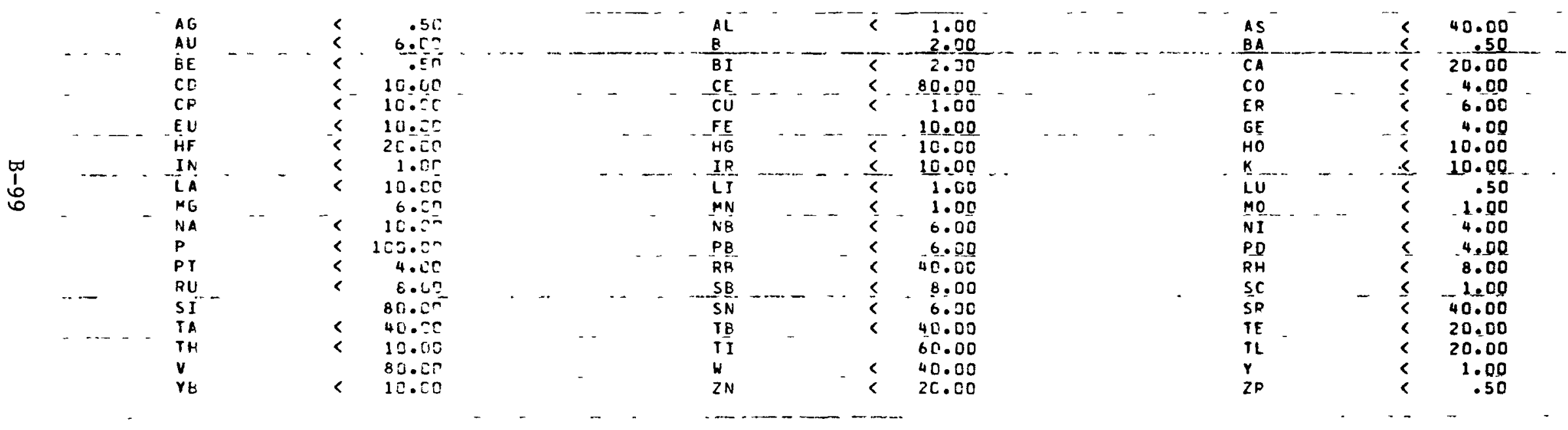

CONCENTRATION BASEO ON ORGINAL SARPLF EEFOPE UILUTION WITH CILUENT

C MEANS GREATER THAN

C MEANS LESS THAN THE SENSITIVITYOF THE SPECTROgFaPHIC PROCEDURE USE

RESULTS ARE CORRECT WITHIN A FACTOP OF $40 \%$. (CNE STANDAFO UEVIATION) 
TABLE B-16 (Continued)

CHEMICAL PURITY

LOG 7194-87

SPECTROCHEMICAL REPQRT

\begin{tabular}{l} 
LAB.NO. 49611 \\
SAMPLE: T194-87 M9C R PEPORT TOE AL EEAVAN \\
\hline
\end{tabular}

3273604200 DILUIION: 1.0000

DATE: $6-2-78$

CONCENTRATION PPM

ELEMENT - CONCENTRATION, PPM

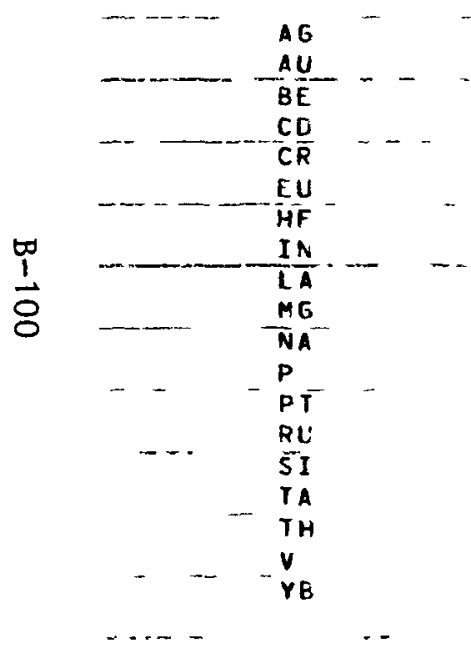

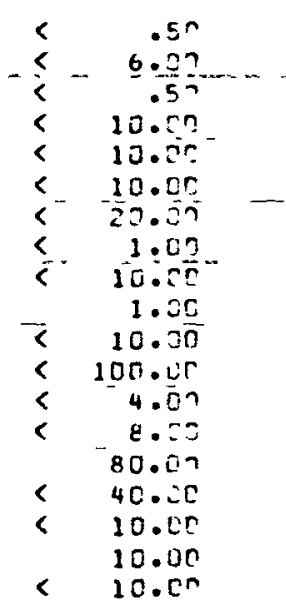

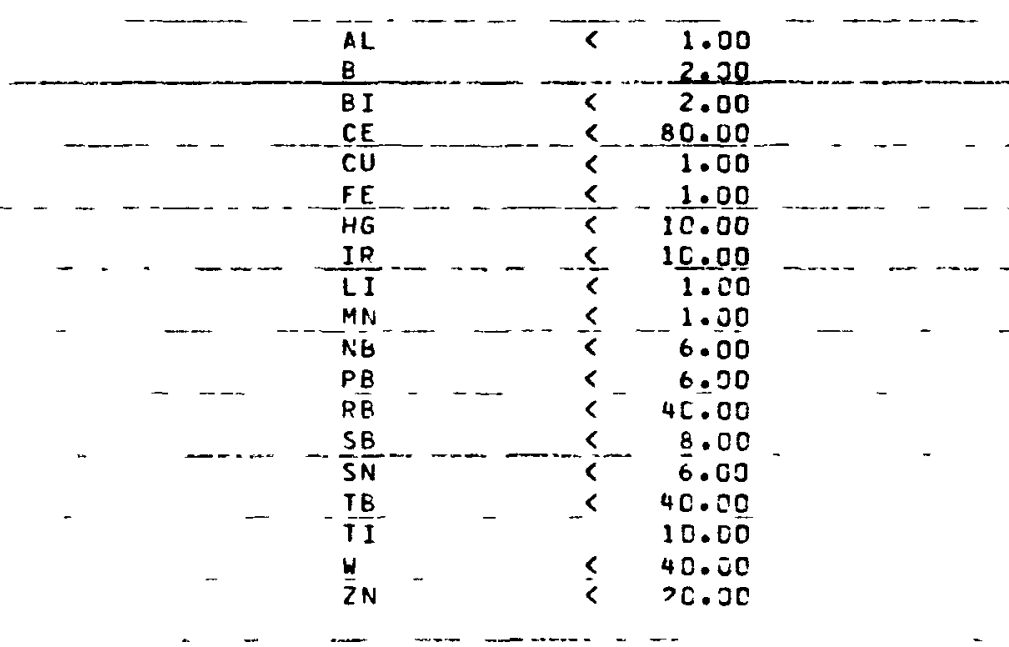

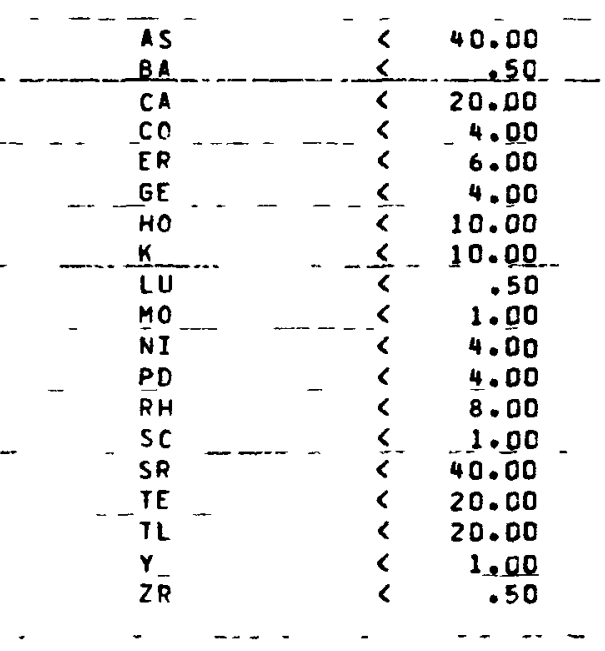

CONCENTRATION BASEO ON ORGINAL SAMPLE BEFORE DILUTION WITH UILUERT

$\checkmark$ MEANS GREATER THAN

< MEANS LESS THAN THE SENSITIVITYOF THE SPECTROGRAPHIC PPOCEOURE USEC

REESLLTS ARE CORRECT WITHIN A FACTOR OF 408 , IONE STANDARÓLEVIATION) 